WSRC-TR-95-0135

Publication Date: May 1995

\title{
Magnetic Survey of the R-Area Bingham Pump Outage Pits ${ }^{(U)}$
}

Westinghouse Savannah River Company

Savannah River Site

Aiken, SC 29808

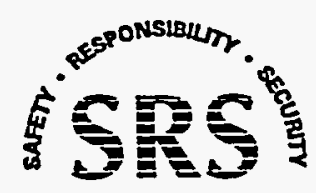

Prepared for the U.S. Department of Energy under Contract No. DE-AC09-89SR18035 


\section{DISCLATMER}

This report was prepared as an account of work sponsored by an agency of the United States Government. Neither the United States Covernment nor any agency thereof, nor any of their employees, makes any warranty, express or implied, or assumes any legal liability or responsibility for the accuracy, completeness, or usefulness of any information, apparatus, product, or process disclosed, or represents that its use would rot infringe privately owned rights. Reference herein to any specific commercial product, jrocess, or service by trade name, trade:nark, manufacturer, or otherwise does not necessarily constitute or imply its endorsement, recommendation, or favoring by the United States Government or any agency thereof. The views and opinions of authors expressed herein do not necessarily state or reflect those of the United States Government or any agency thereof.

This report has been reproduced directly from the best available copy.

Available to DOE and DOE contractors from the Office of Scientific and Technical Information, P.O. Box 62, Oak Ridge, TN 37831; prices available from (615) 576-8401.

Available to the public from the National Technical Information Service, U.S. Department of Commerce; 5285 Port Royal Road, Springfield, VA 22161.

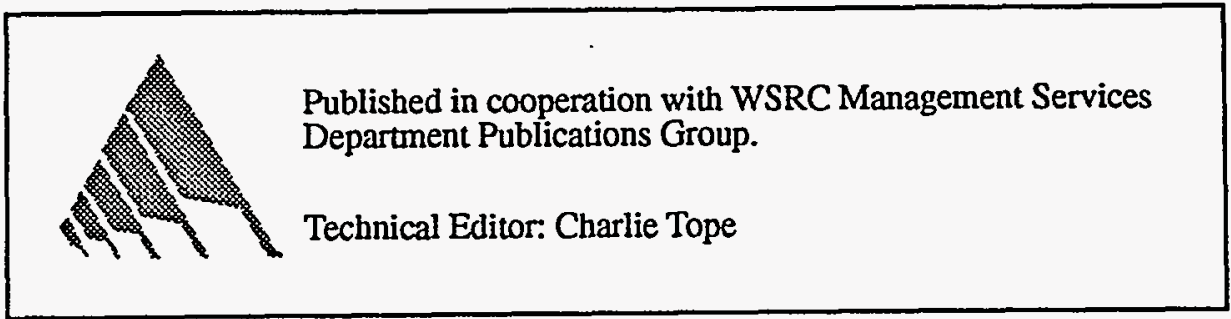




\section{DISCLAIMER}

Portions of this document may be illegible in electronic image products. Images are produced from the best available original document. 


\section{Magnetic Survey of the R-Area Bingham Pump Outage Pits $(U)$}

R. J. Cumbest

Site Geotechnical Services 
This page intentionally left blank. 


\section{Contents}

Executive Summary

Introduction and Scope 3

Magnetic Survey Concepts...................................................... 3

Description of Survey Site ..................................................... 5

Magnetic Survey................................................................................7

Survey and Analytical Techniques .......................................... 7

Magnetic Data Set .......................................................................... 7

Discussion and Conclusions.

Bibliography

\section{Appendix A-R-Area Bingham Pump Outage Pit}

Magnetic Survey Data.

\section{List of Figures}

Figure 1. Location of Reactor Areas on SRS

Figure 2. Location of the R-Area Bingham Pump Outage Pits in relation to R-Area facility

Figure 3. Typical temporal variations exhibited by the total magnetic field due to diumal variation,micropulsations, and magnetic storms on the sun (adapted from Breiner, 1973)

Figure 4. Superposition of the earth's ambient magnetic field and anomalous fields produced by induced monopoles and dipoles to produce anomalies in the total field (adapted from Breiner, 1973)

Figure 5. The relationship of magnetic inclination and declination to geographic directions on the Earth (adapted from Telford and others, 1978)

Figure 6. Typical profiles of magnetic field intensity across a monopole and dipole for conditions similar to SRS. Also shown is a contour representation of magnetic field intensity over a dipole with accompanying representative profiles (adapted from Breiner; 1973) 
Figure 7. Magnetic intensity profiles across several geometric shapes of interest for magnetic field inclination similar to that at SRS (i.e. 60 degrees) (adapted from Breiner, 1973)

Figure 8. The intensity of the total magnetic field compared with the vertical gradient over a prismatic body (adapted from Breiner, 1973)

Figure 9. Model total field and vertical gradient magnetic profiles for single 55-gallon steel drums in vertical position with the top at various distances below the sensor. Profiles calculated-for a total field intensity of $53220 \mathrm{nT}$ and northern hemisphere inclination of 70 degrees (adapted from Gilkeson and others, 1992)

Figure 10. Contour representation of total field anomaly calculated for a single 55-gallon steel drum in vertical position with the top $10 \mathrm{ft}$ below the sensor. Calculation based on a total field intensity of $53220 \mathrm{nT}$ and northem hemisphere inclination of 70 degrees (adapted from Gilkeson and others, 1992)

Figure 11. Contour representation of cache of five steel 55-gallon drums in a 12-ft by $12-\mathrm{ft}$ area with the top of the drums $10 \mathrm{ft}$ below the sensor. Calculation based on a total field intensity of $53220 \mathrm{nT}$ and northern hemisphere inclination of 70 degrees (adapted from Gilkeson and others, 1992)

Figure 12. Planimetric map of the R-Area Bingham Pump Outage Pits Magnetic Survey Arẹa

Figure 13. Station locations for the R-Area Bingham Pump Outage Pits Magnetic Survey. Station locations marked by " + "

Figure 14. Color contoured R-Area Bingham Pump Outage Pits magnetic survey diumal corrected top sensor data

Figure 15. Color contoured R-Area Bingham Pump Outage Pits magnetic survey diumal corrected bottom sensor data

Figure 16. Color contoured R-Area Bingham Pump Outage Pit magnetic survey vertical gradient data

Figure 17. Color contoured R-Area Bingham Pump Outage Pit magnetic survey vertical gradient data with inferred trench boundaries outlined 


\section{Executive Summary}

The R-Area Bingham Pump Outage pits are a series of parallel trenches in which construction materials were buried. The suspected locations of three of the trenches are marked by HP orange waste unit marker balls, in addition to concrete pit monuments that were placed based on historical information. In order to confirm the locations of the trenches and to evaluate the presence of other buried ferric material, a magnetic survey was conducted at the R-Area Bingham Pump Outage Pits. The survey is defined by lines spaced $10 \mathrm{ft}$ apart oriented subperpendicular to the trench elongation direction, with survey stations spaced at 5 -ft intervals along each line. This resulted in a $10-\mathrm{ft}$ by 5 -ft rectangular grid node pattern.

The magnetic survey resolved at least four linear arrays of magnetic field and gradient anomalies that result from buried ferric material. Three of the linear arrays correspond to suspected trench locations as delineated by HP orange marker balls and pit monuments. However, a fourth linear anomaly occurs between two of the suspected trenches, indicating the presence of an unmarked trench. In addition to linear anomalies that are associated with the trenches, other isolated anomalies occur due to ferric sources that can be identified on the surface, such as signs, monuments, and metal pipes. 
This page intentionally left blank.

- 


\section{Introduction and Scope}

Several of the reactor areas on Savannah River Site (Figure 1) have burial pits located outside their perimeters that contain miscellaneous construction equipment. The material contained in these trenches is a consequence of major modifications to the reactor's primary and secondary cooling water systems, and consists of such items as pipes, cables, ladders, drums, and boxes of miscellaneous hardware (see WSRC-RP-91-1203, 1994). During the modifcations the reactors were offline and this period was called the Bingham Pump Outages. The trenches containing the construction material associated with the modifications are termed the Bingham Pump Outage Pits, although these pits do not contain the actual Bingham pumps.

The Bingham Pump Outage Pits for R Reactor are located outside of the perimeter fence on a small hill northeast of the facility (Figure 2). In order to confirm the locations and edges of the trenches and to identify other possible buried material in the area, a magnetic survey was conducted by the Site Geotechnical Services Department at the request of the Environmental Restoration Department during September 1994. The survey consisted of a grid over and in areas adjacent to the waste unit markers associated with the pit.

\section{Magnetic Survey Concepts}

The magnitude of the magnetic field measured near the Earth's surface is influenced by several factors. The main contribution to the total intensity is due to the internal magnetic field of the earth produced in the Earth's core. However, this field is modified by the solar wind of charged particles produced by electromagnetic activity on the Sun. This solar wind distorts the magnetic field produced by the Earth into a tear drop shape with the blunt end pointing toward the Sun. More importantly, the effect of the solar wind is to produce time variations in the total field. The Earth's rotation, in effect, introduces a diurnal variation with a 24-hour period. Also, superimposed on this well-defined 24-hour cycle are variations with no well-defined period, produced by the chaotic nature of the Sun's electromagnetic activity. Typically these variations manifest themselves as micropulsations of less than $10 \mathrm{nT}$, but significantly larger variations can be produced by magnetic storms on the Sun (i.e. sunspots). The net results of these variations is illustrated in Figure 3.

The Earth's magnetic field is also modified by features near the surface that produce localized spatial variations, or anomalies, in the field intensity. The use of magnetic techniques in searching for buried metallic objects is a consequence of the ability of iron and iron alloys to cause relatively large amplitude anomalies. These anomalies are produced by two effects. Some materials have properties that allow them to become magnetized in the presence on an external magnetic field. This process is called induction. The external magnetic field is the inducing field, and the field produced by the material is the induced field. The total field (B) now becomes the sum of the inducing external field $(\mathbf{H})$ and the field produced by the material (H'). This relationship can be expressed:

$$
\mathbf{B}=\mathbf{H}+\mathbf{H}^{\prime}
$$

In addition, some materials possess a magnetic field independent of any external source. This field is typically acquired during the history of the material, and is called remnant magnetization. For most magnetic survey applications the magnitude of the induced field is assumed to be much greater than the remnant magnetization. This assumption has been shown to be valid in the specific case of standard 55-gallon metal drums (see Gilkeson and others, 1992).

In reality, magnetic fields always are manifest as dipoles; that is, north and south magnetic poles occur in pairs to produce dipole fields. Field line diagrams of dipole and monopole fields are presented in Figure 4. In this type of representation, the density of the field lines is proportional to the field magnitude. To a first approximation, the magnetic field produced near the Earth's core can be considered a single dipole, with magnetic field lines impinging the surface of the Earth at 90 degrees at the north and south magnetic poles, and being parallel to the surface of the Earth at the magnetic equators.

The geometric relationship of the magnetic field lines to the geographic poles is expressed by magnetic declination and inclination. Declination describes the angular relationship of the component of the field line parallel to the surface of the Earth, to geographic north. The magnetic inclination expresses the angular relationship between the magnetic field lines and the horizontal (see Figure 5). Although these angular relationships change slowly, due to poorly understood long term variations, the declination in the vicinity of SRS is about 4 degrees to the west and the inclination about 65 degrees.

Although inducing bodies usually possess highly irregular shapes and sizes, they can be usefully modeled as dipoles or arrays of dipoles. In some instances, if the area of consideration is near one end of a dipole relative to the dipole separation, the local field can be considered to be pro- 
duced by a single pole, and approximates monopole behavior. The anomalous fields produced by a dipole and monopole for a magnetic inclination of 60 degrees in the northern hemisphere (i.e., conditions very similar to SRS) and their resultant effect on the total magnetic field measured near the body are illustrated in Figure 4 . Note that the anomalous field lines on the north side of the dipole are opposite in sense to the ambient field, therefore reducing the field strength in this location. In contrast, the anomalous field lines on the south side are in the same sense, resulting in an increased field strength. The monopole exhibits similar behavior although not as extreme: This phenomena explains the asymmetrical magnetic highs and lows produced by inducing bodies at mid-latitudes on the Earth. Figure 6 illustrates typical magnetic profiles that would be observed at the latitude of SRS over monopoles and dipoles, in addition to a generalized contour representation of a dipole, with illustrative profiles across the contours.

Generalized magnetic field intensity profiles across more complicated shapes, for an inclination of 60 degrees, are illustrated in Figure 7. These profiles all exhibit the typical asymmetrical signatures along the north.- south profiles, with magnetic highs occurring over southern portions of the bodies.

The previous discussion has been presented in the context of the intensity of the total magnetic field. However, another measurement that can be very useful in interpreting magnetic anomalies is the vertical magnetic gradient, which changes much more rapidly with distance than the total field. This effect results in edge-enhancement of anomalies and more effective anomaly separation (see Figure 8). The vertical magnetic gradient can be determined by making measurements of the total magnetic intensity at two known elevations. The vertical gradient is then determined by the difference between the two measurement, divided by their separation. The two measurements can be made by a single sensor instrument by moving the sensor between the two measurements, or by an instrument with two separated sensors.

To conduct a magnetic survey, magnetic data are collected at discrete intervals either along a traverse or over twodimensional (2-D) grid. In practice, data for 2-D grids are usually collected as multiple traverses: Traverse data can be conveniently displayed as magnetic intensity profiles. Two-dimensional grid data are usually contoured and displayed as contour diagrams. Displayed in this fashion, these data can be qualitatively interpreted by comparing them with profiles or contours that would be predicted from bodies of known shape and size.
In addition to qualitative information, profiles and contour diagrams may also be used for quantitative analysis if the magnetic response of specific objects is known. Because of their widespread use in the past as containers for industrial materials, 55-gallon steel drums are commonly targeted during waste site characterization. Gilkeson and others (1992) evaluated the magnetic response from single and multiple drums buried at different depths. The magnetic response captured in a model calibrated from actual measurements on a 55-gallon drum. These anomalies were calculated for a total field intensity of $53220 \mathrm{nT}$ and inclination of 70 degrees, conditions very similar to SRS.

Figure 9 models total field and vertical gradient profiles over a single drum, in vertical position, with the drum top at different depths below the sensor. If single, deeply buried drums are the target, these profiles make clear the need to locate the sensor near the ground surface in order to minimize the distance between the sensor and drum. For sensor - drum distances greater than about $20 \mathrm{ft}$ the anomaly amplitude has decreased to values that may not be discernible from noise.

Figure 10 illustrates the contour of an anomaly for a single drum with its top located $100 \mathrm{ft}$ below the sensor. Figure 10 also shows the station locations of a typical 5-ft by 5-ft grid over the anomaly. Note that using a $20-\mathrm{ft}$ by 20 -ft grid may fail to detect the anomaly at all, and that a poorly placed $10-\mathrm{ft}$ by 10 -ft grid would only provide a maximum amplitude of $25 \mathrm{nT}$. This amplitude may not be discernible from noise and, at best, would offer poor resolution concerning the drum location.

Figure 11 shows the contour of a total field magnetic anomaly for a cache of five drums in a 12-ft by 12-ft area with the drum tops $10 \mathrm{ft}$ below the sensor. Note again that a $20-\mathrm{ft}$ by $20-\mathrm{ft}$ grid may fail to detect the anomaly. A $10-\mathrm{ft}$ by 10-ft grid would crudely resolve the anomaly. However, Gilkeson and other's (1992) demonstrated that at a depth of $10 \mathrm{ft}$, if the center drum were removed the drums on the comers would be resolved into individual anomalies. If this type of information is required from the magnetic survey, then 5 -ft grid node separations would be needed to fully resolve the individual anomalies associated with each drum.

In addition to the above considerations, other factors enter into the design of an effective magnetic survey. Although smaller grid spacings allow better resolution of anomalies they are also labor intensive.. Placing the sensor near the ground makes it much more sensitive to small, shallow sources, such as metallic trash or magnetite variations in the soil. This is especially a problem for gradiometer surveys because the effect is amplified. In addition, gradiom- 
eters are most effective when the sensor separation is onefifth less the distance to the target. If the sensors are placed near the ground, this condition may not be met for shallow target. Careful consideration of what information is needed from the magnetic survey, and the resources available, will determine which aspects discussed above are most important.

\section{Description of Survey Site}

A planimetric map of the R-Area Bingham Pump Outage Waste Unit is presented in Figure 12. The waste unit, as delineated by the HP. Waste Unit marker balls, and the immediately surrounding area, is a relatively flat grassy area bordered on all sides by pine forest.

The most prominent objects in the survey area are twelve orange HP waste unit marker balls that occur in rectangular patterns that delineate the corners of suspected burial trenches. Also, 6 concrete posts (monuments) occur between the orange HP marker balls near the end of each rectangular area. These monuments presumably mark the centerlines of the suspected trenches. In addition to these objects, large metal signs occur at each end of the suspected trenches, and several smaller, metal "Underground Radiation" signs occur along the margins of the waste unit. In some locations metal objects, such as rebar and iron rails, can be seen protruding from the suspected trench locations. Also, a pile of metal pipes can be seen at the western edge of the survey area. 


\section{:}

This page intentionally left blank. 


\section{Magnetic Survey}

\section{Survey and Analytical Techniques}

The grid established for the magnetic survey of the R-Area Bingham Pump Outage Pits (see Figure 13) is defined by survey lines spaced $10 \mathrm{ft}$ apart and oriented subperpendicular to the trench elongation direction to allow maximum resolution of the trench boundaries. Measurements were taken at 5-ft intervals along the survey lines to yield a 5-ft by $10-\mathrm{ft}$ rectangular grid node pattern. The grid is located to give coverage to the area enclosed by and outside of the waste unit marker balls. In addition, the magnetic survey grid was extended to the wooded area to the northeast of the waste unit to investigate an area that appeared to be suspect, based on aerial photography and previously acquired radar data. The grid station locations were established using a transit to mark 90-degree angles, and a measuring tape marked at 5-ft intervals to determine distances.

Magnetic data were collected with an EG\&G Geometrics G-856 Gradiometer, with the top sensor at 1.22 meters (4 $\mathrm{ft}$ ) from ground level and the bottom sensor 0.61 meters ( 2 $\mathrm{ft}$ ) from ground level. This configuration places the sensors near the ground to maximize the sensitivity to small near-surface or deeply-buried objects. This instrument has a nominal accuracy of $0.1 \mathrm{nT}$. However, high magnetic gradients $(2600 \mathrm{nT} / \mathrm{m})$ sharply degrade the signal. In most cases, this degredation deteriorate the accuracy to $1 \mathrm{nT}$. More severe degradation can result in significant accuracy losses, resulting in poor data very near large metallic objects. As a quality assurance/quality control check, and to determine diumal variations, a single station was occupied several times during the survey.

The data were downloaded from the magnetometer through a RS-232 port to an IBM PC Model 80. After downloading, the data were assigned coordinates and diurnal corrected with MAGLOC, software provided by EG\&G. Further data display and processing, including gridding and contouring were accomplished with software provided by GEOSOFT Inc. Data gridding was accomplished with BIGRID, which is specifically designed to grid line data and enhance trends. The data were filtered with a 20-ft low pass filter along each traverse line before gridding. This allowed very effective attenuation of wavelengths of $5 \mathrm{ft}$ or less in the data in the traverse direction. Magnetic gradients were calculated by subtracting the top sensor reading from the bottom sensor reading and dividing by their separation. Since the top and bottom sensor readings are taken at a relatively short time scale (approxi- mately 3 seconds) relative to diumal variations, the gradient is calculated from the data before diurnal correction. The data for the survey along with daily diumal variations are tabulated in the Appendix A.

\section{Magnetic Data Set}

The magnetic survey data are displayed in Figures 14 to 16. Magnetic field and gradient anomalies occur in areas on and near the HP waste unit marker balls, the monuments, the metal signs, and other metal objects that can be seen on the surface. However, the most prominent magnetic features occur as four, parallel, linear arrays of anomalies aligned with the long dimension of the waste unit. Three of these linear arrays fall within the suspected trench boundaries as defined by the HP. Waste unit marker balls and the presence of centerline monuments. The linear anomaly arrays marked by the HP waste unit markers consist of two short arrays (approximately $300 \mathrm{ft}$ long) and a longer array to the northeast of the short arrays that is approximately $500 \mathrm{ft}$ long.

The fourth linear array of magnetic anomalies falls in the interval between the northwestern-most linear array and the linear array marked by the HP waste unit markers in the middle. Several of the anomalies defining the arrays exhibit very high magnetic field gradients in localized areas.

The area investigated by the magnetic survey to the northeast of the waste unit (the area northeast of the long trench) contains several isolated, small, low amplitude anomalies. However, these anomalies -are significantly smaller than the anomalies associated with the linear arrays. 
This page intentionally left blank. 


\section{Discussion and Conclusions}

The magnetic survey of the R-Area Bingham Pump Outage Pits indicates the presence of four linear arrays of magnetic field and gradient anomalies. The discontinuous nature of the anomalies that compose the linear arrays, in addition to the linear geometry of their arrangement, is consistent with the presence of ferric objects and groups of objects buried in trenches. Based on the vertical gradient data, the inferred approximate boundaries of the four trenches associated with the linear arrays are shown in Figure 17. Three of the trenches correspond closely with the locations defined by the HP arange waste unit marker balls and centerline monuments. However, the magnetic data indicate the presence of a fourth, unmarked trench of intermediate length that occurs parallel and adjacent to the longest trench. Power spectrum analyses of the anomalies indicate source depths of from 0 to $10 \mathrm{ft}$. The shallowest depth can be confirmed by the presence in several locations of metallic material protruding from the ground.

In addition to the linear anomaly arrays that are associated with the trenches, several isolated anomalies are present. Most of these anomalies can be attributed to ferric objects on the surface, such as metal signs, HP orange waste unit marker balls, and trench centerline monuments. Although investigation of the area northeast of the longest trench revealed several small isolated anomalies, indicating the presence of buried ferric material, the low amplitude and small size of these anomalies indicate that they do no represent objects of any significant size. These anomalies probably result from metallic trash near the surface, such as metal cans, etc. The data do not indicate that the trenches are present in this area. 
This page intentionally left blank. 


\section{Bibliography}

Breiner, S. 1973, Applications Manual for Portable Magnetometers, Sunnyvale, California 94084.

Gilkeson, R. H., S. R. Gorin, and D. E. Laymon, 1992, "Application of Magnetic and Electromagnetic Methods To Metal Detection". Proceedings of the Symposium on the Application of Geophysics to

- Engineering and Environmental Problems, Volume 1, pp. 309-328.

Sexton, G. B., and R. J. Pirkle, 1993, Final Report, Ground Pentetrating Radar Investigations at the Bingham Pump Outage Pits, Savannah River Site, October 1993.

Telford, W. M., L. R. Geldart, R. E. Sheriff, and D. A. Keys, 1978, Applied Geophysics, Cambridge University Press, 860 p.

Westinghouse Savannah River Company, 1994, RI Work Plan for K-Area Bingham Pump Outage Pit, USDOE Report WSRC-RP-91-1203, Savannah River Site, Aiken, SC 29808. 
This page intentionally left blank. 


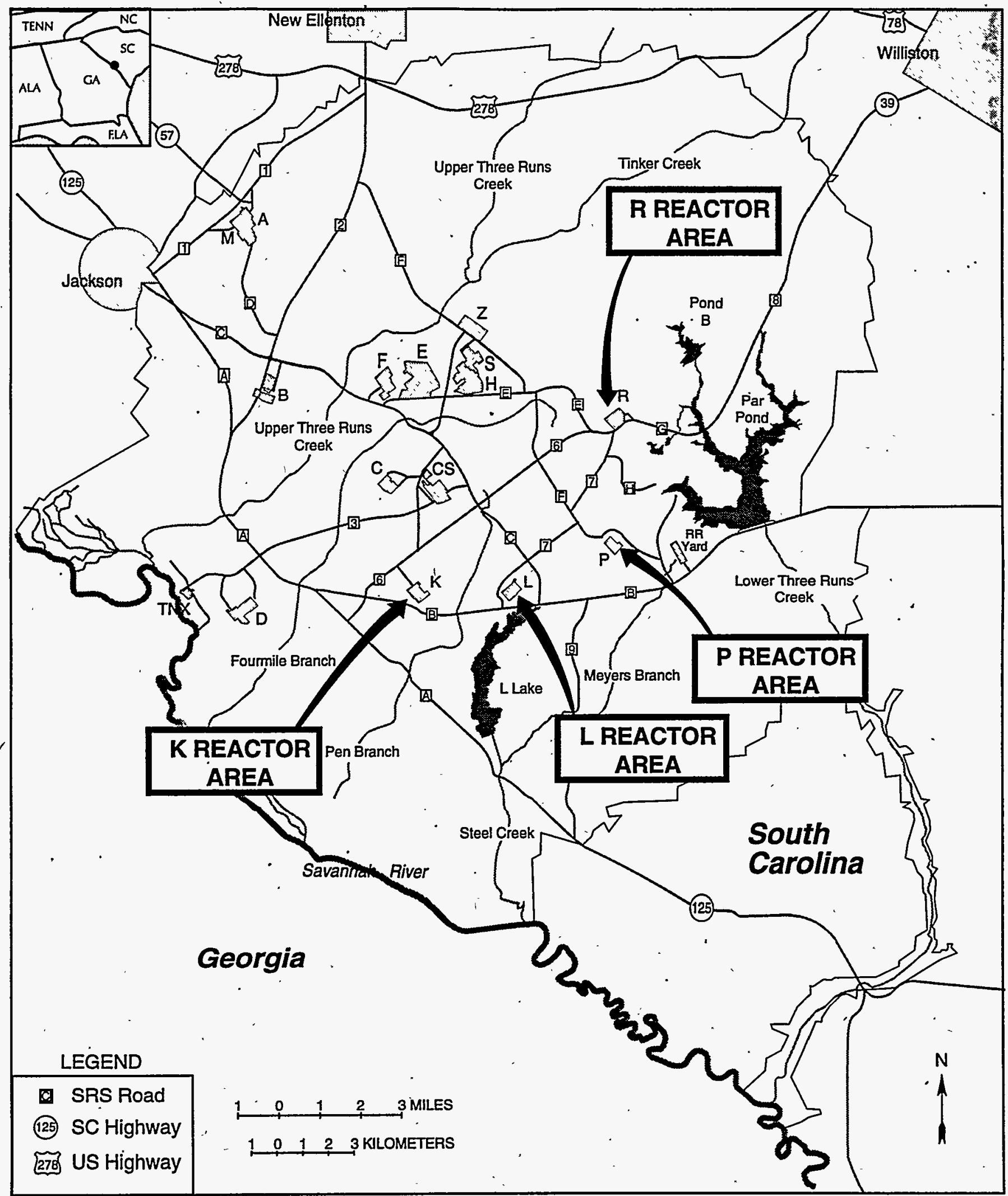

Figure 1. Location of Reactor Areas on SRS 


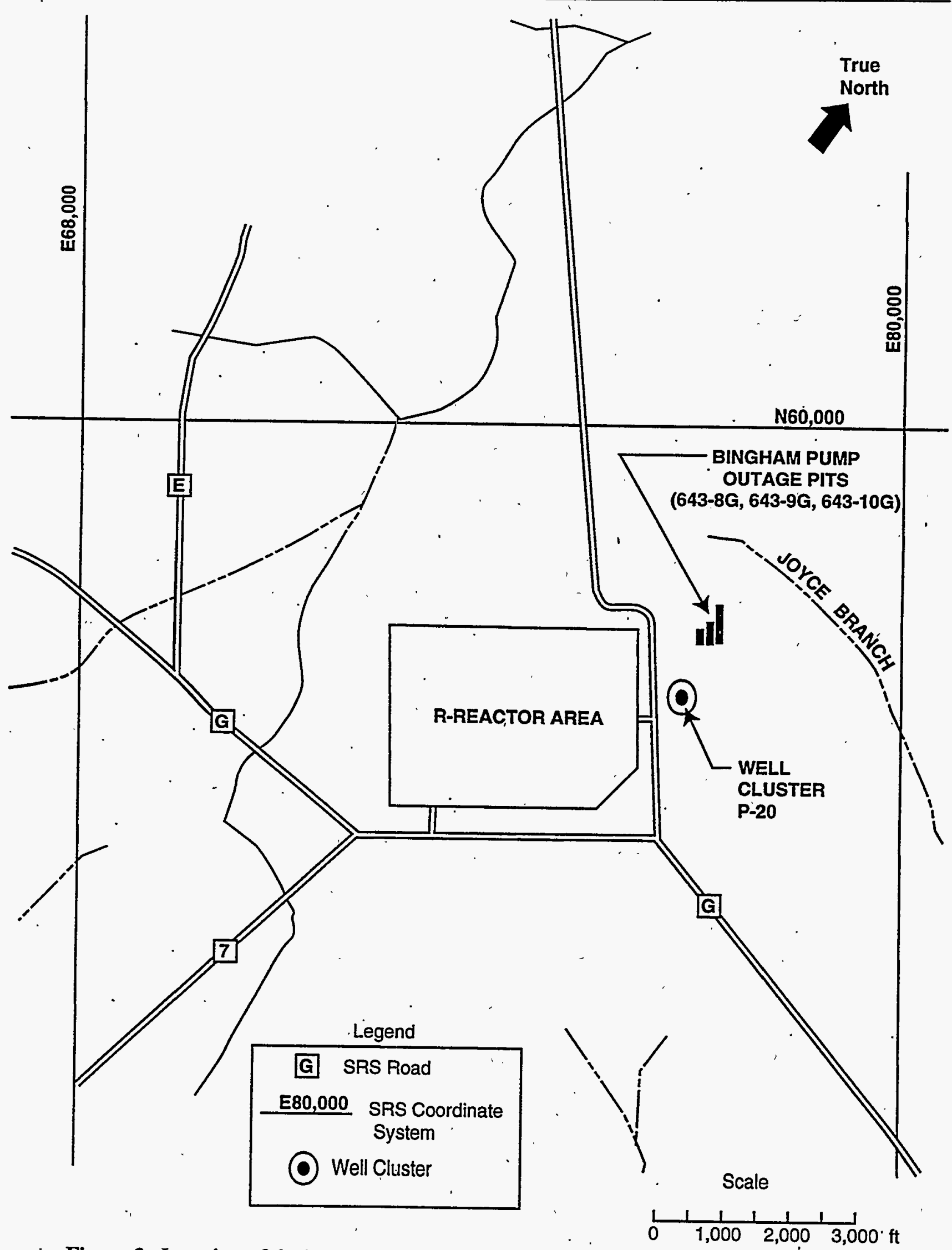

Figure 2. Location of the R-Area Bingham Pump Outage Pits in relation to R-Area facility 


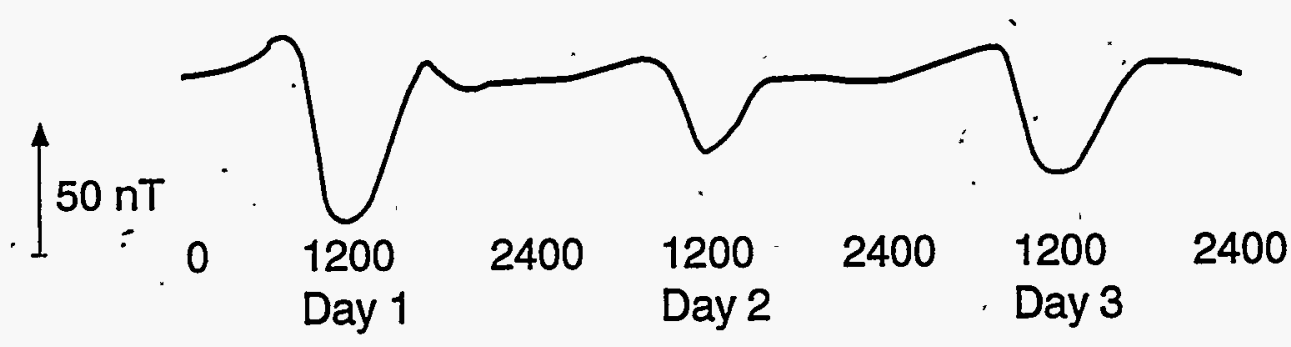

Diurnal Variation

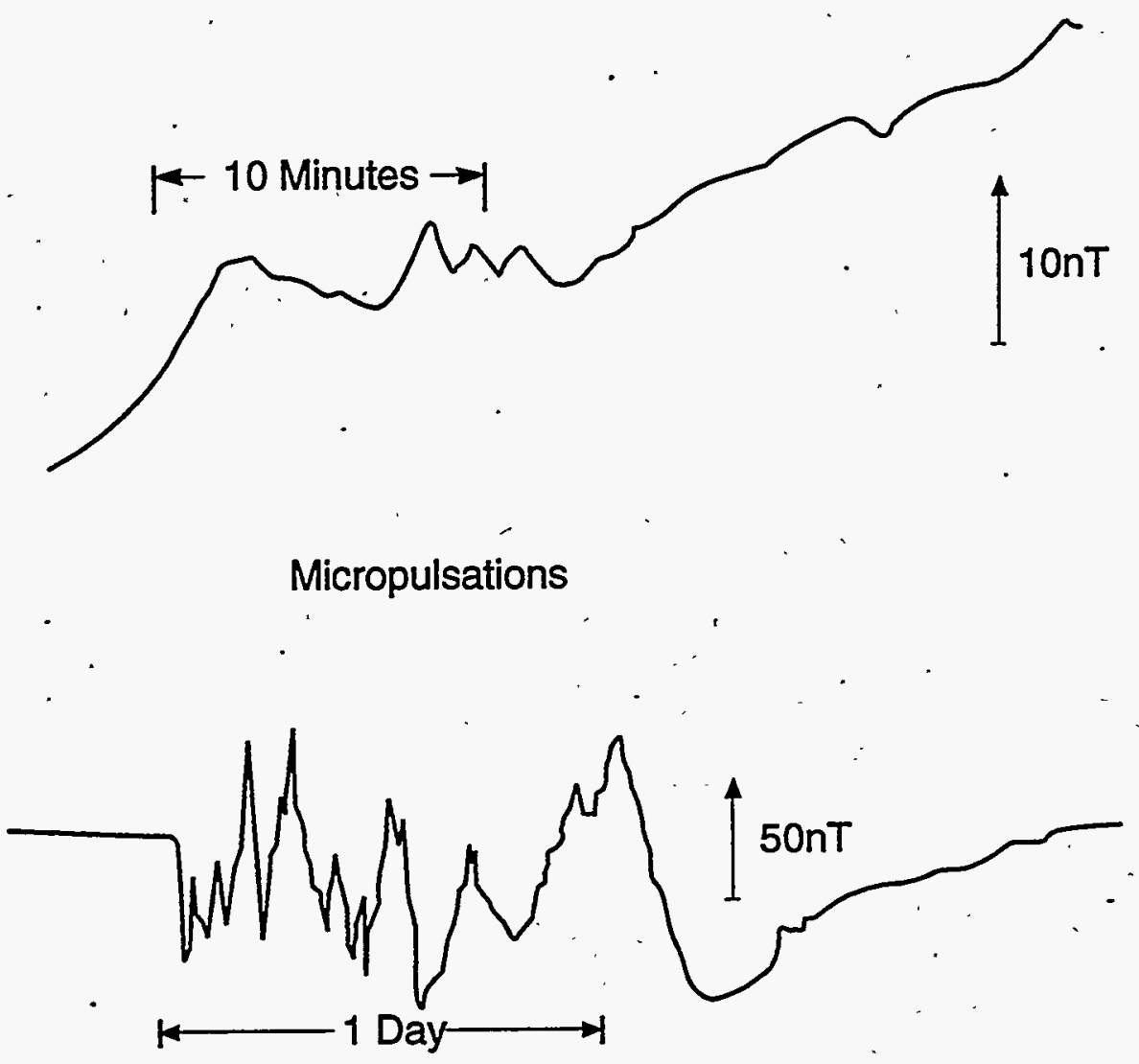

Magnetic Storm

Figure 3. Typical temporal variations exhibited by the total magnetic field due to diurnal variation, micropulsations, and magnetic storms on the sun (adapted from Breiner, 1973) 

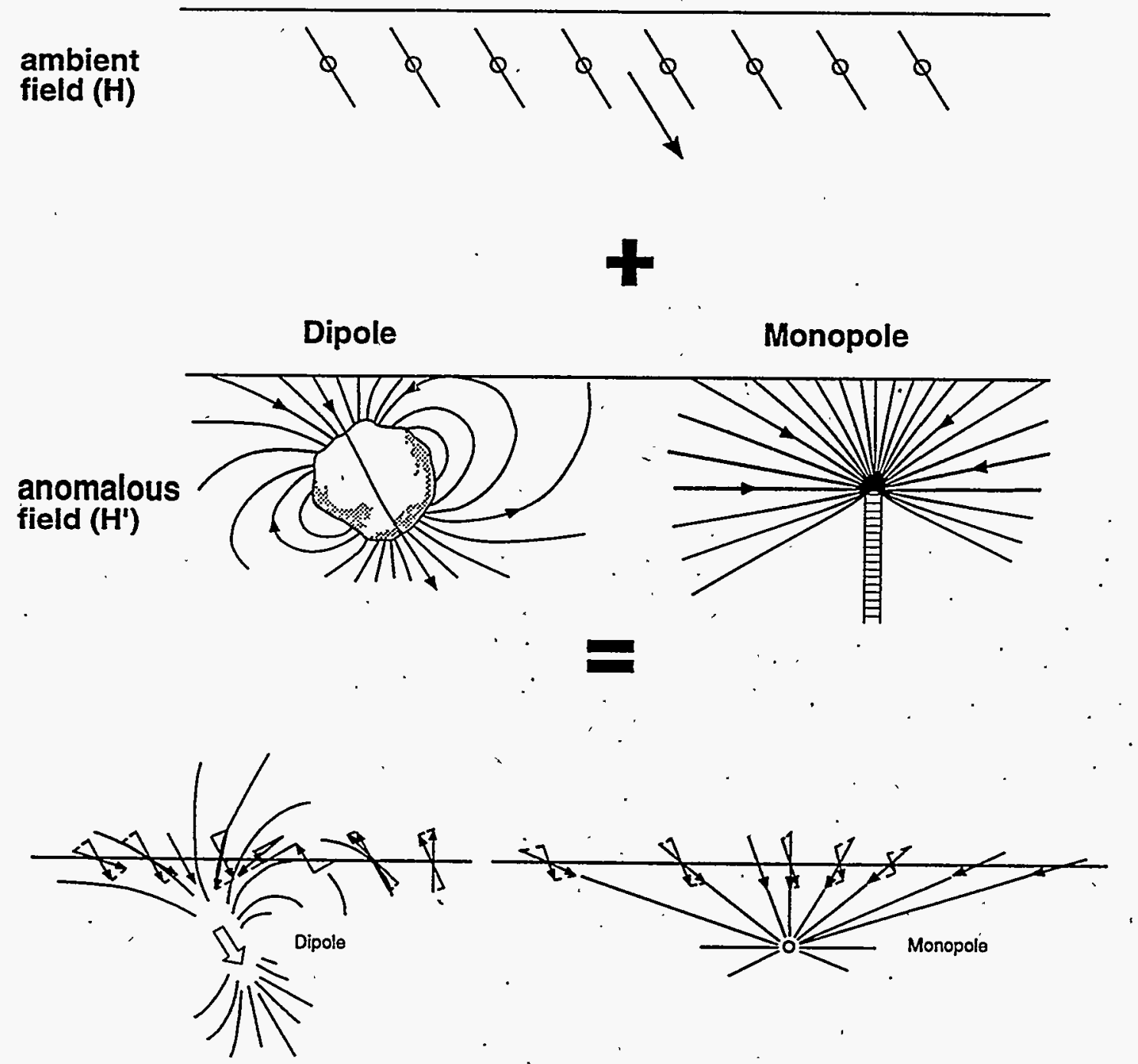

\section{total obseryed field (B)}


Figure 4. Superposition of the Earth's ambient magnetic field and anomalous fields produced by monopoles and dipoles to produce anomalies in the total fields (adapted from Breiner, 1973) 


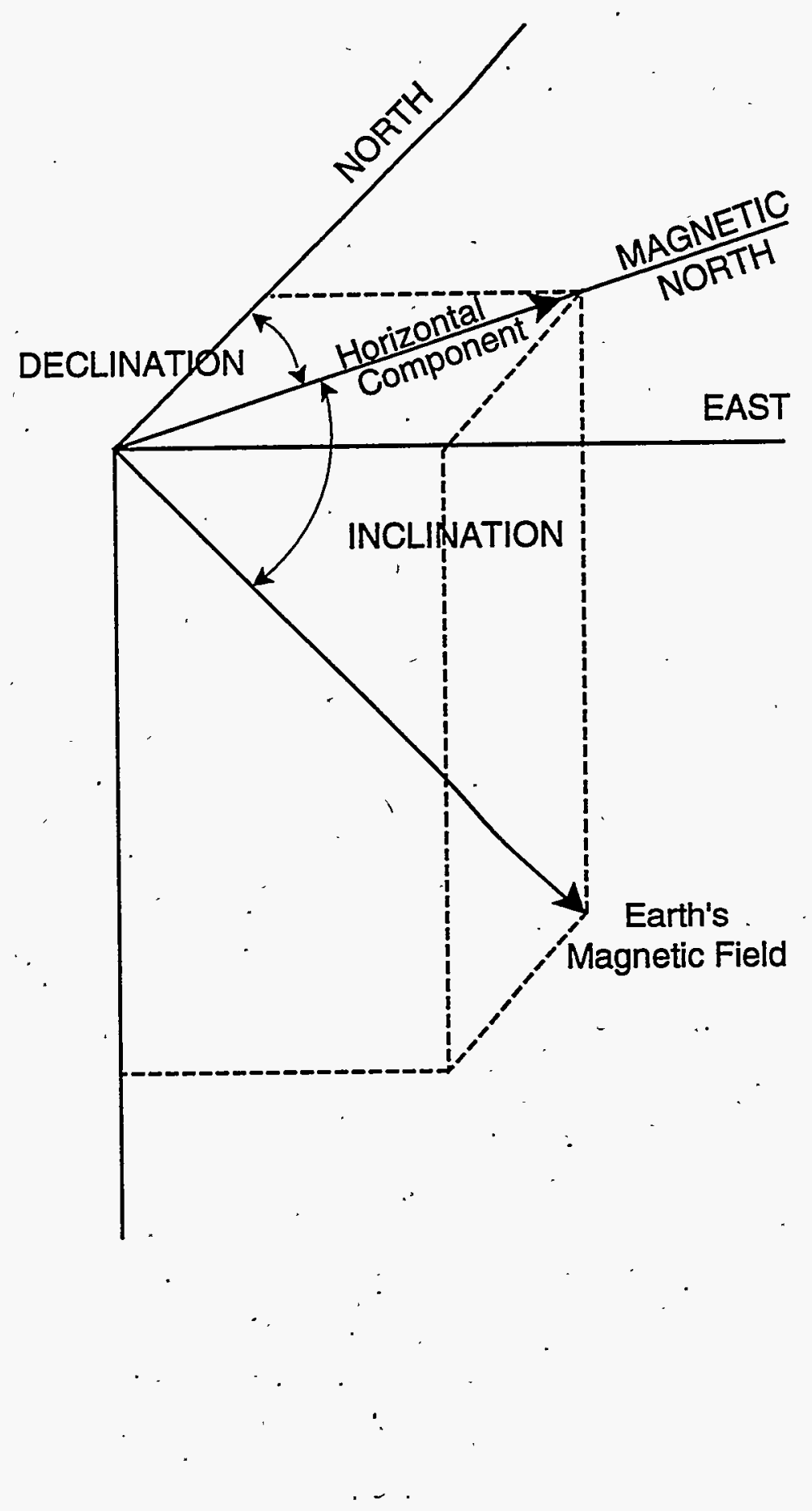

Figure 5. The relationship of magnetic inclination and declination to geographic directions on the Earth (adapted from Telford and others, 1978) 

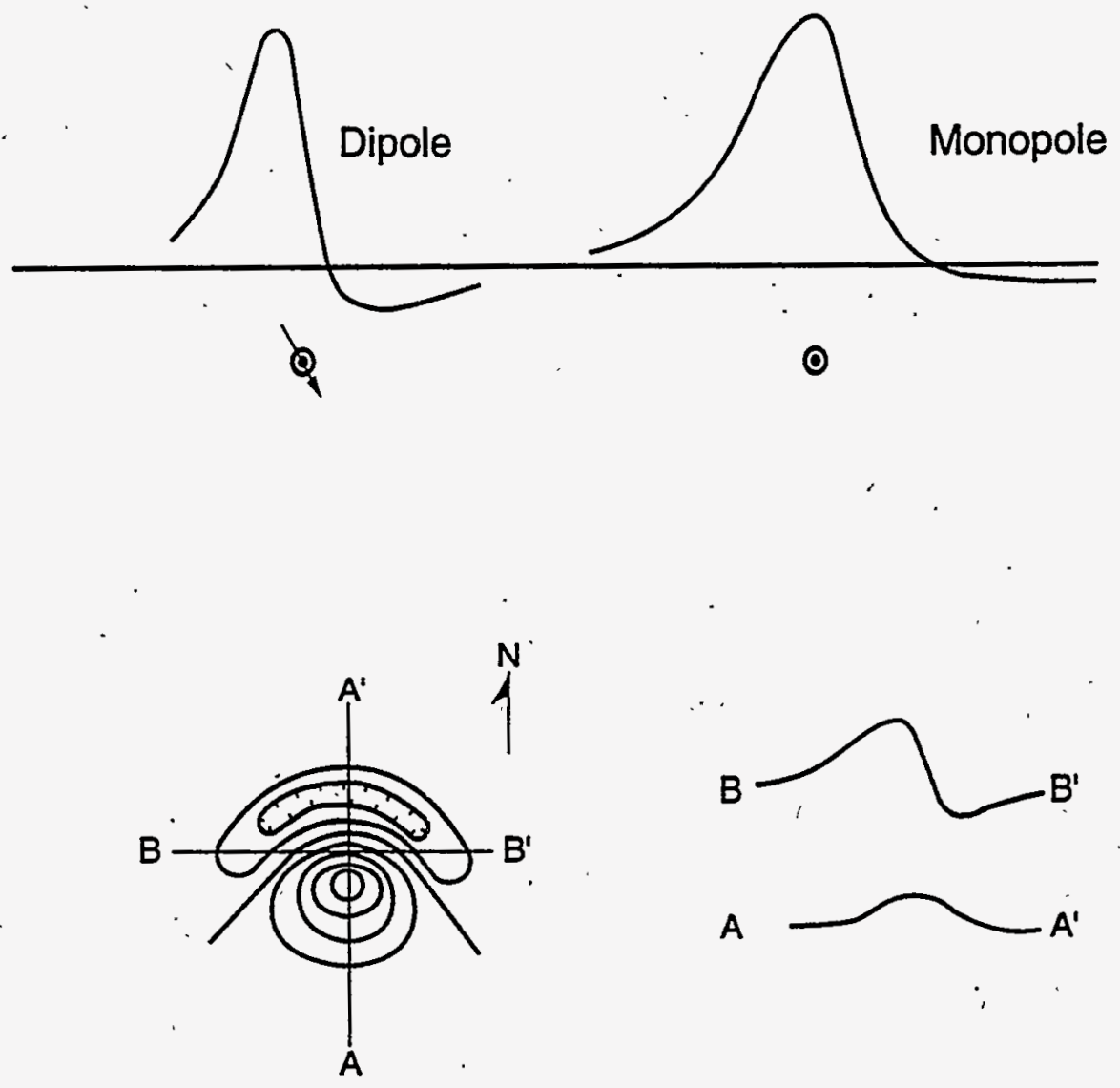

Figure 6. Typical profiles of magnetic field intensity across a monopole and dipole for conditions similar to SRS. Also shown is a contour representation of magnetic field intensity over a dipole with accompanying representative profiles (adapted from Breiner, 1973) 


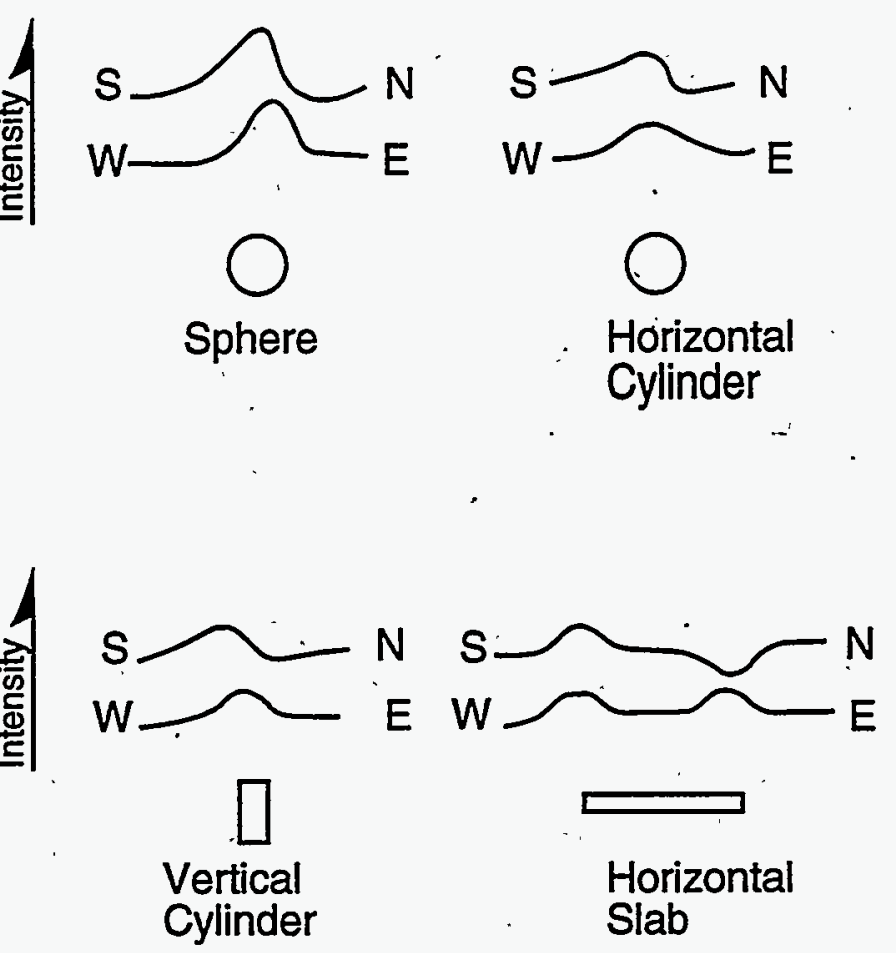

Figure 7. Magnetic intensity profiles across several geometric, shapes of interest for magnetic field inclination similar to that at SRS (i.e. 60 degrees) (adapted from Breiner, 1973) 


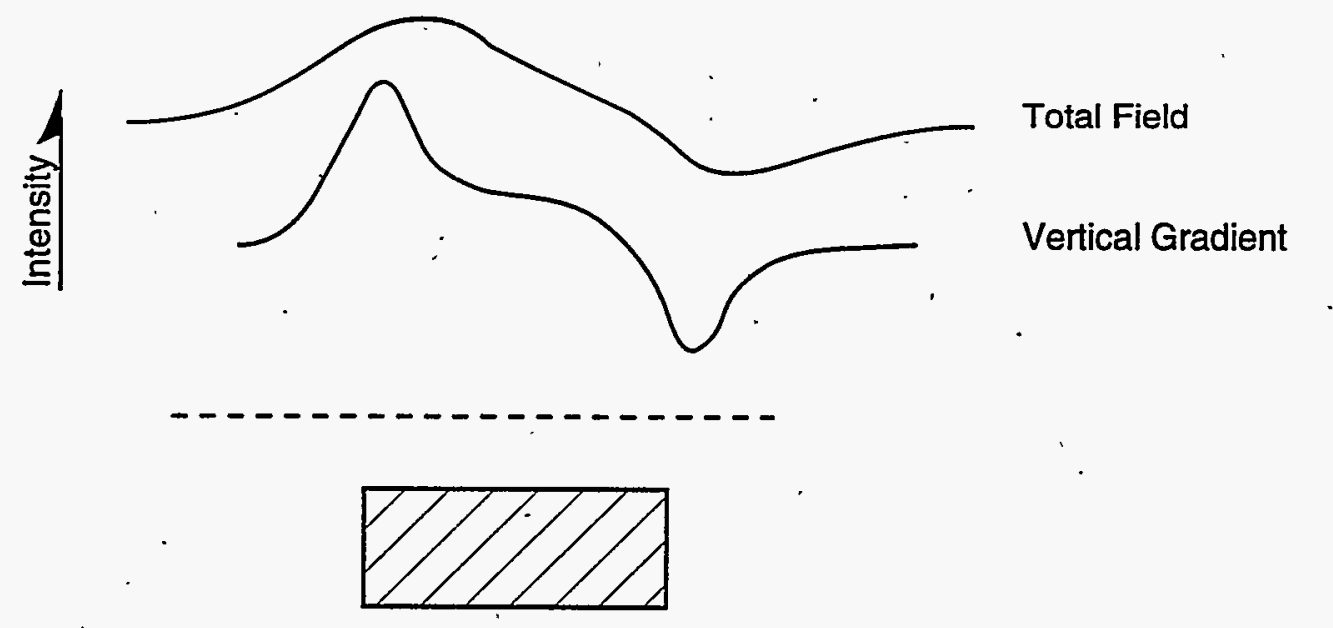

Figure 8. The intensity of the total magnetic field compared with the vertical'gradient over a prismatic body (adapted from Breiner, 1973) 


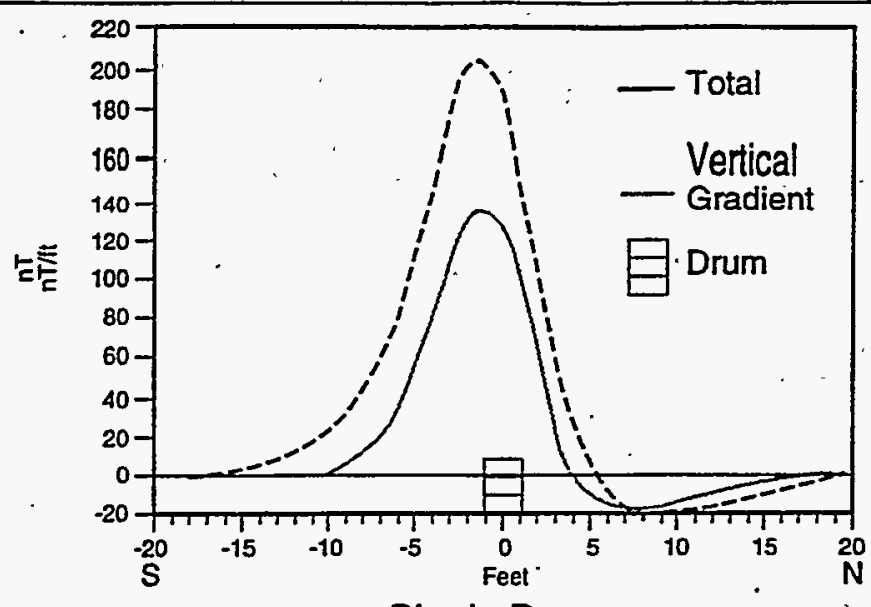

Single Drum

$(6 \mathrm{ft})$

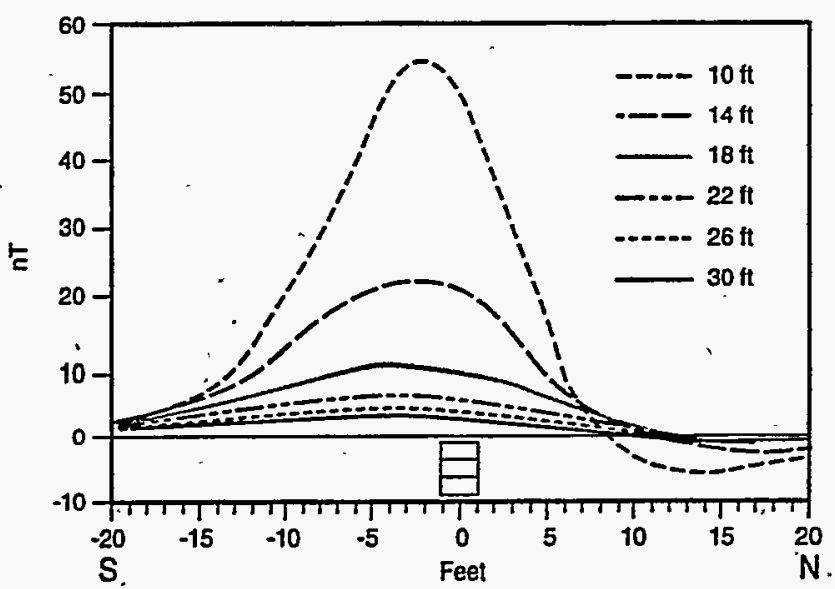

Total Field

Single Drum

$(10-30 \mathrm{ft})$

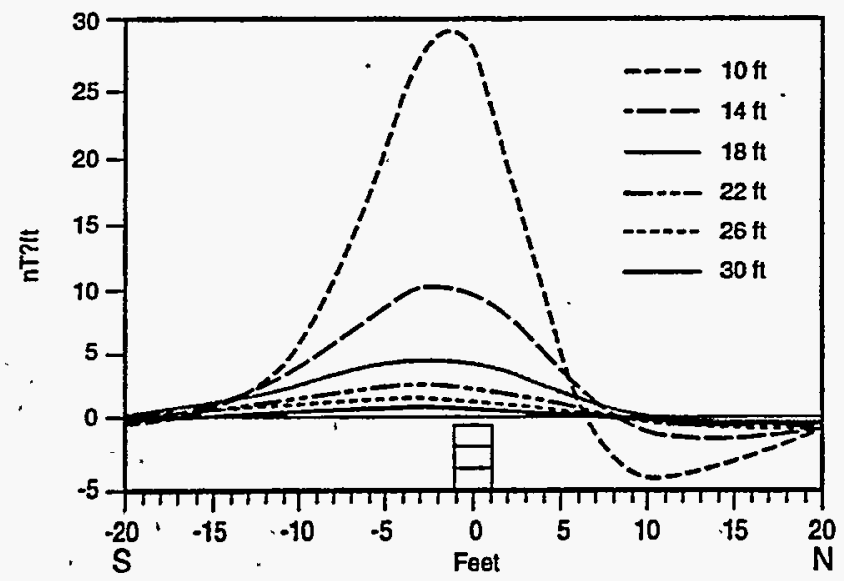

Gradient

Single Drum.

(10-30 ft)

Figure 9. Model.total field and vertical gradient magnetic profiles for single 55-gallon steel drums in vertical position with the top at various distances below the sensor. Profiles calculated for a total field intensity of $53220 \mathrm{nT}$ and northern hemisphere inclination of 70 degrees (adapted from Gilkeson and others,1992) 


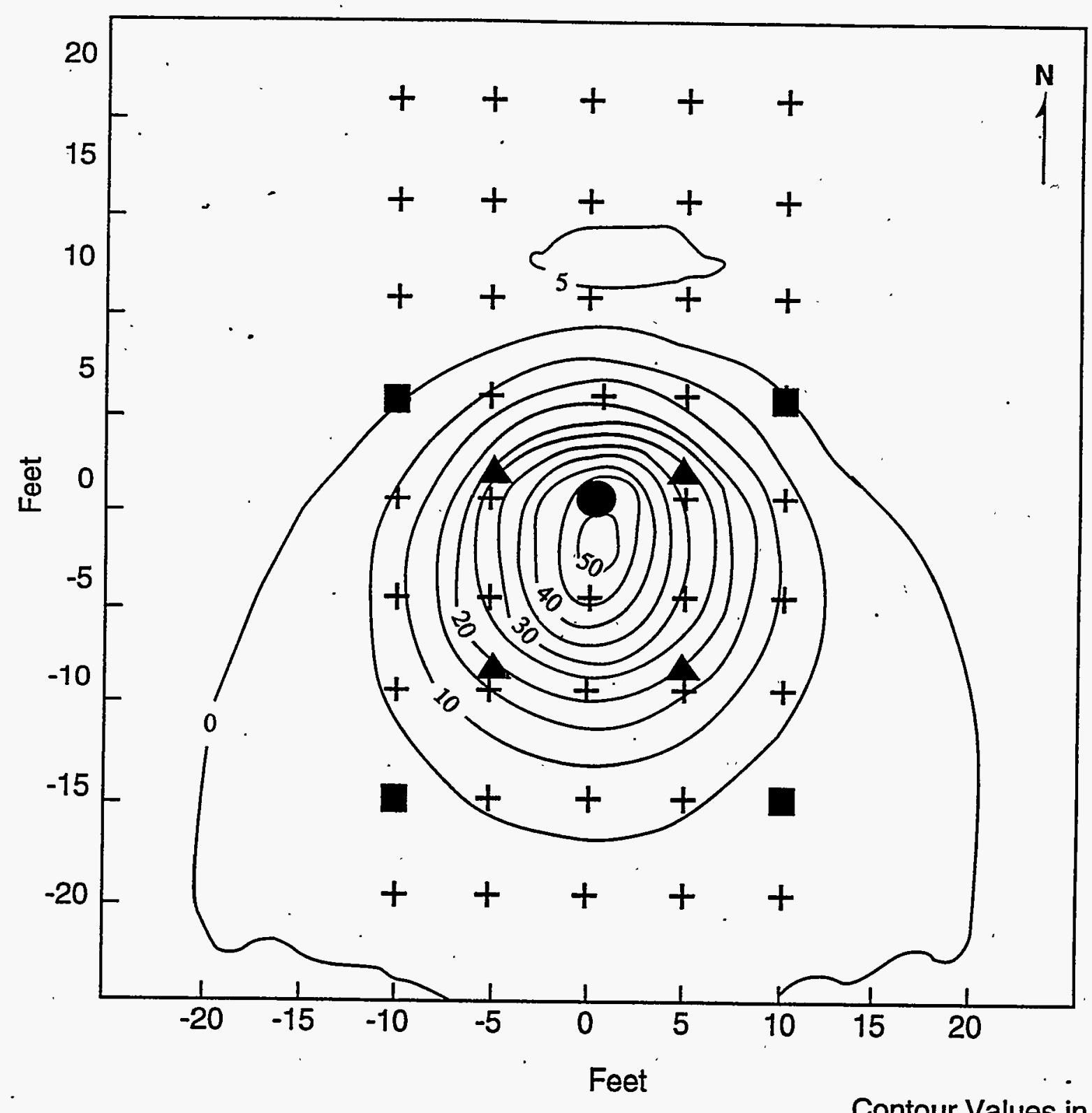

Contour Values in $\mathrm{nt}$



Figure 10. Contour representation of total field anomaly calculated for a single 55-gallon steel drum in vertical position with the top $10 \mathrm{ft}$ below the sensor. Calculations based on the total field intensity of $53220 \mathrm{nT}$ and northern hemisphere inclination of 70 degrees (adapted from Gilkeson and others, 1992) 


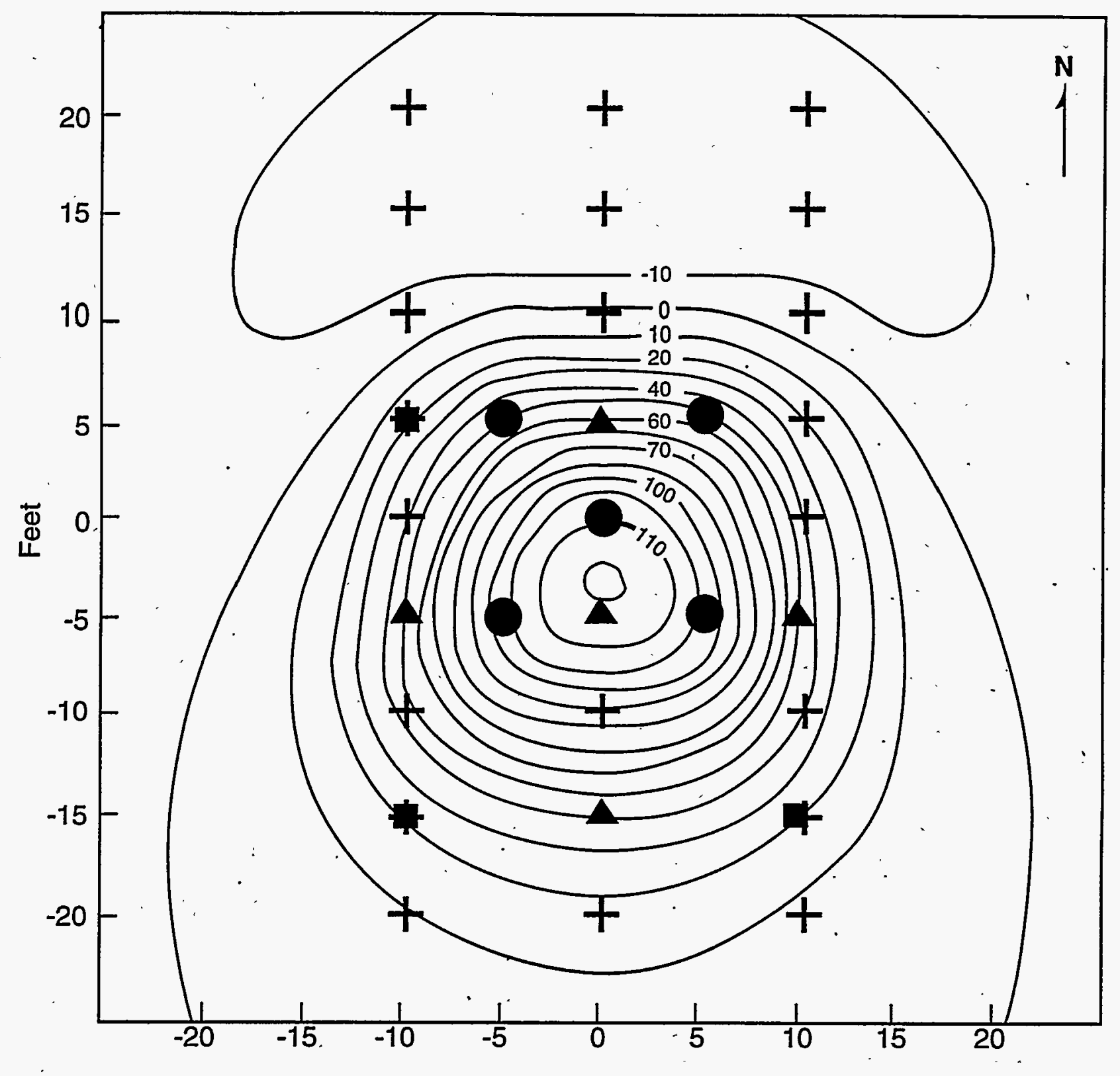

Feet

Contour Values in nt

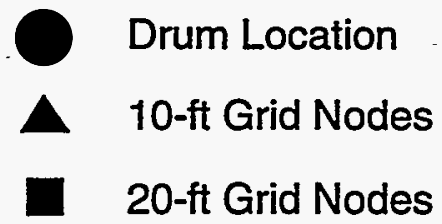

Figure 11. Contour representation of cache of five steel 55-gallon drums in a 12 -ft by $12-\mathrm{ft}$ area with the top of the drums $10 \mathrm{ft}$ below the sensor. Calculation based on a total field intensity of $53220 \mathrm{nT}$ and northern hemisphere inclination of 70 degrees (adapted from Gilkeson and others, 1992) 


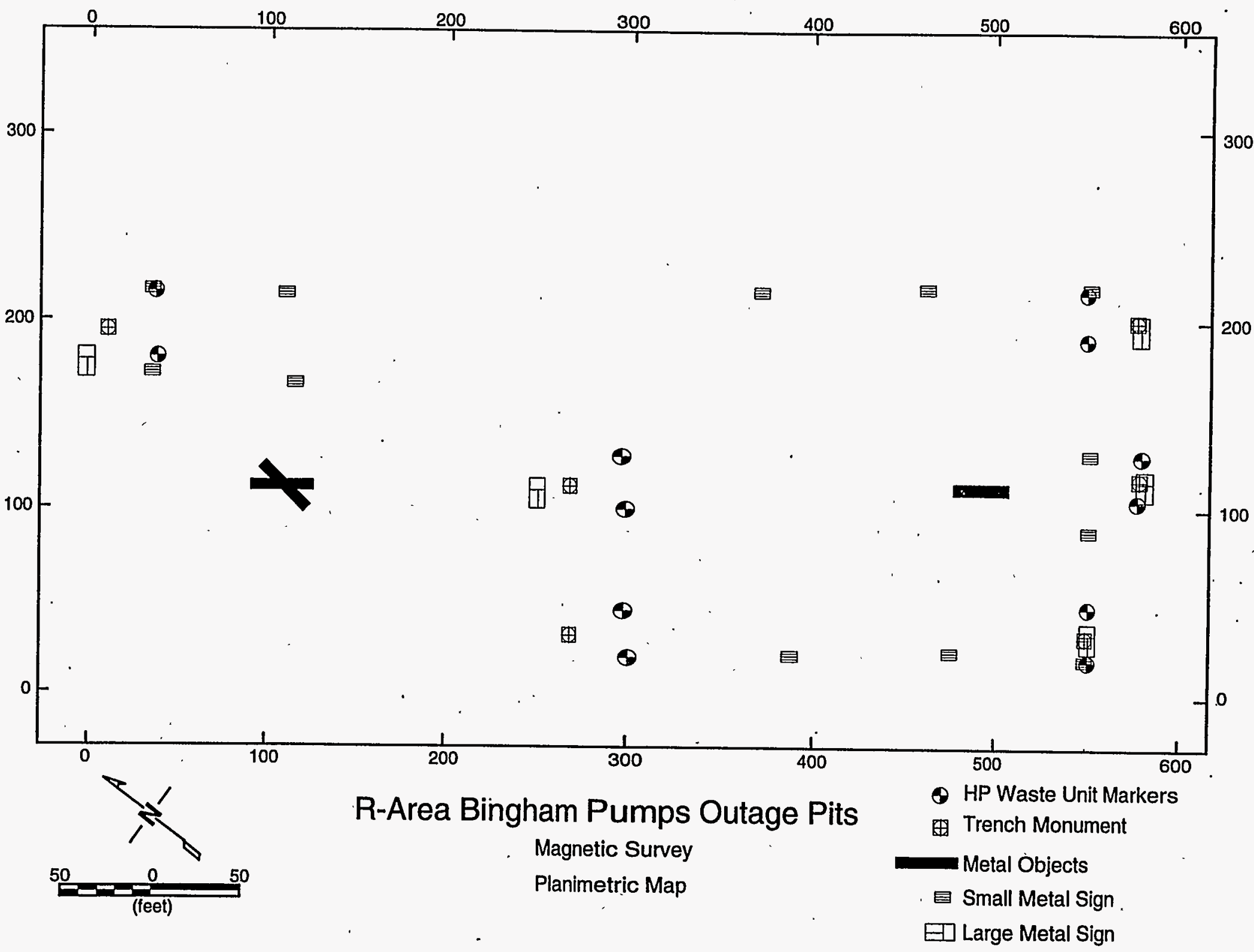

Figure 12. Planimetric map of the R-Area Bingham Pump Outage Pits Magnetic Survèy Area 


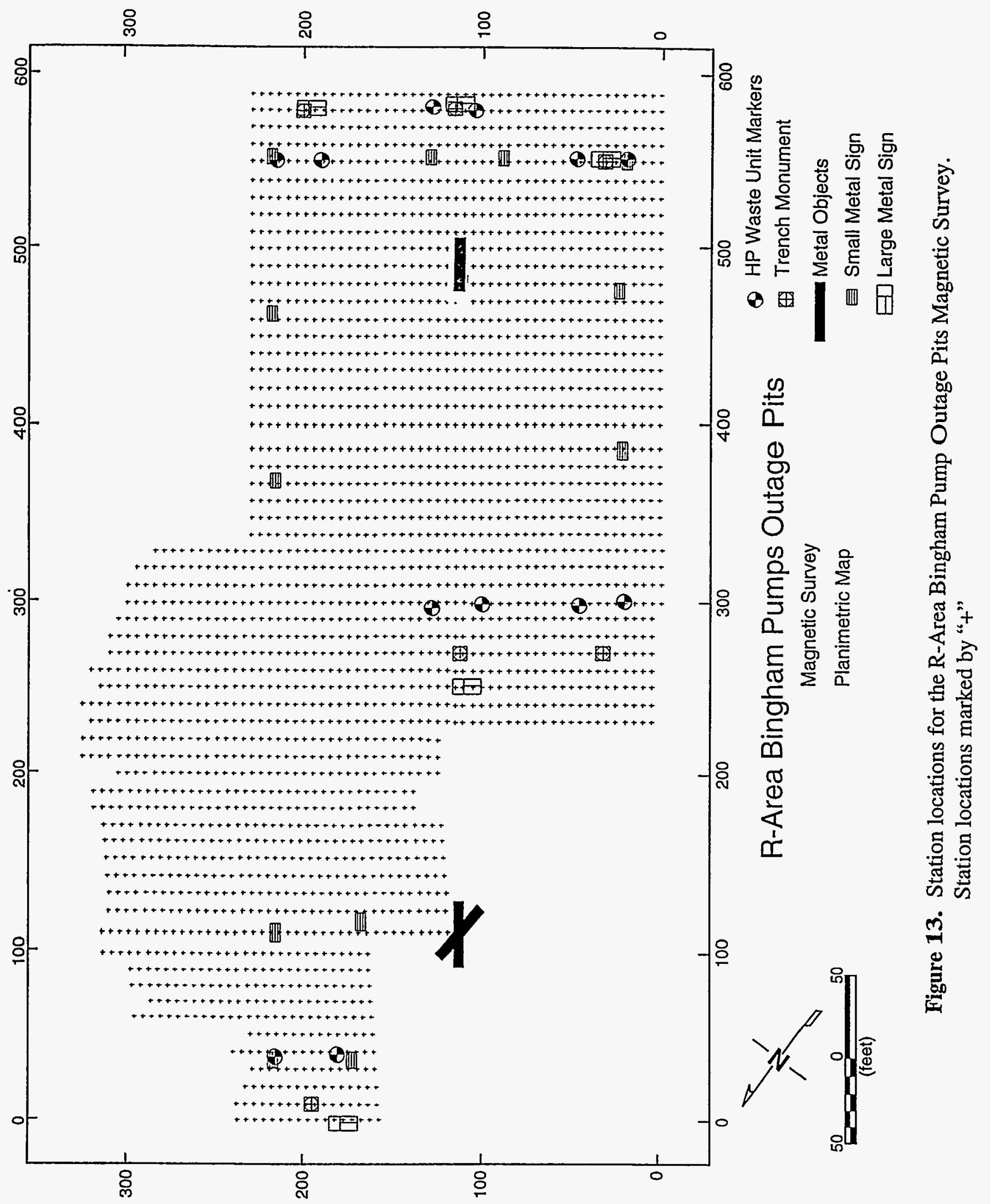




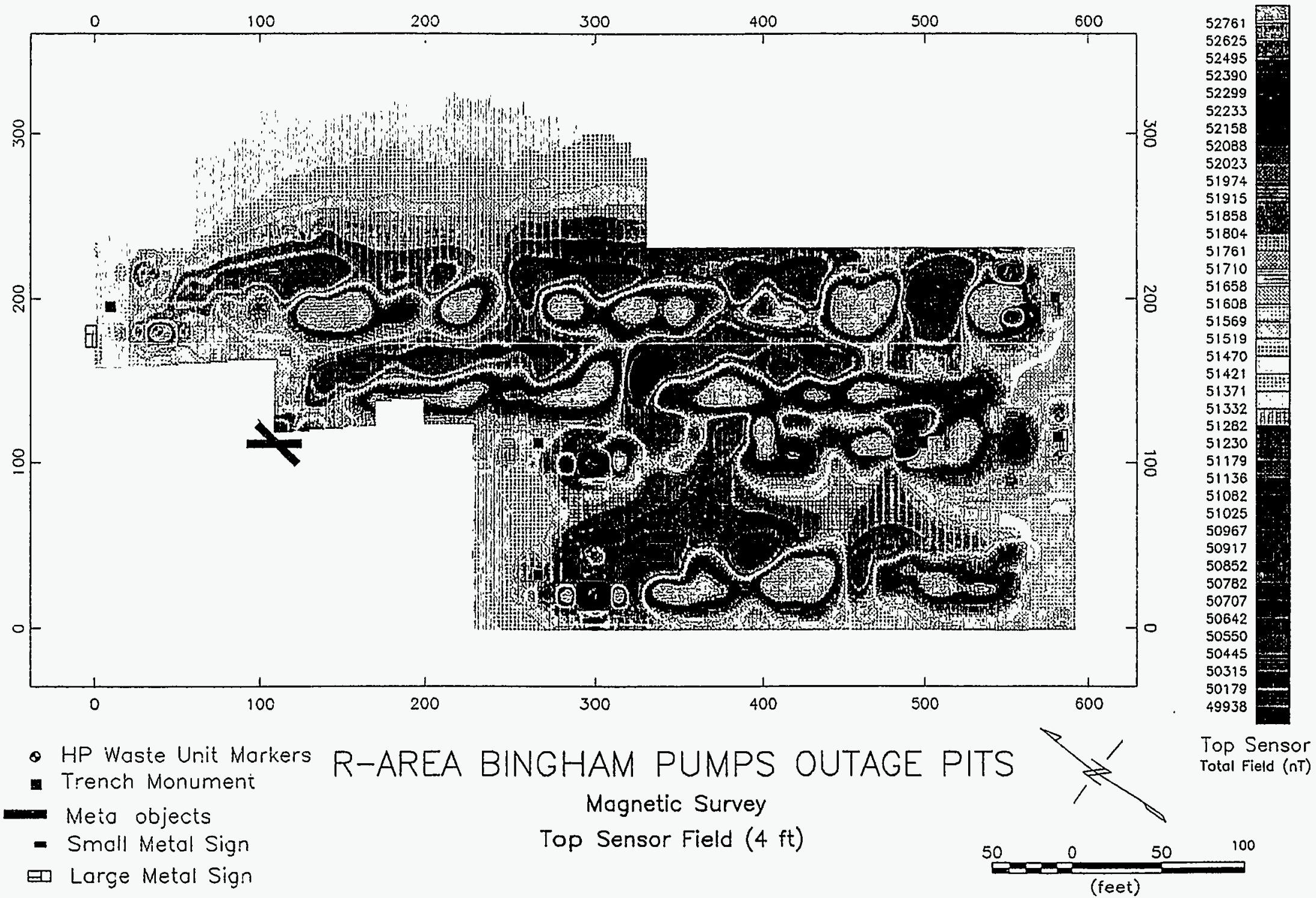

Figure 14. Color contoured R-Area Bingham Pump Outage Pits magnetic survey diurnal corrected top sensor data 


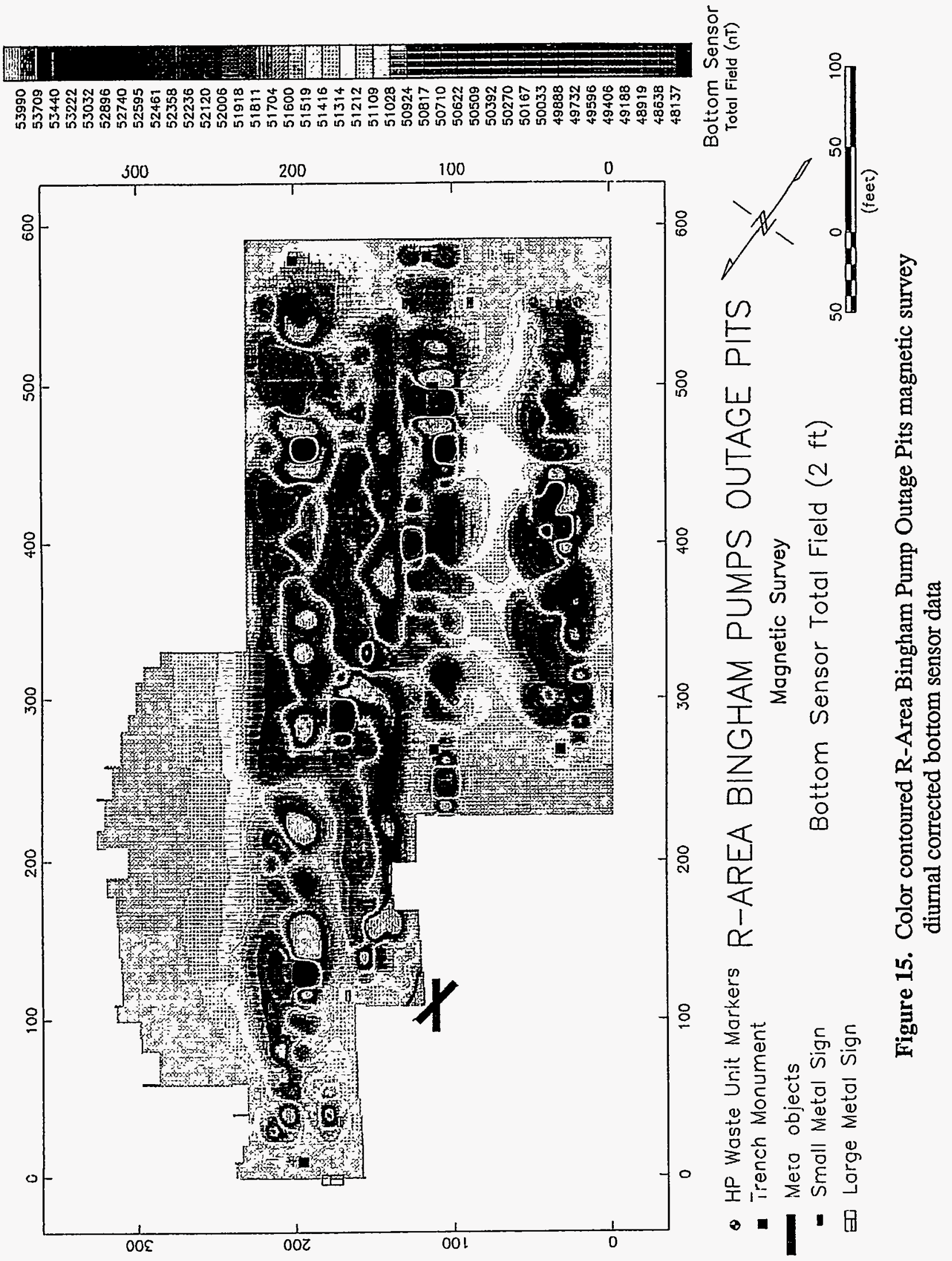








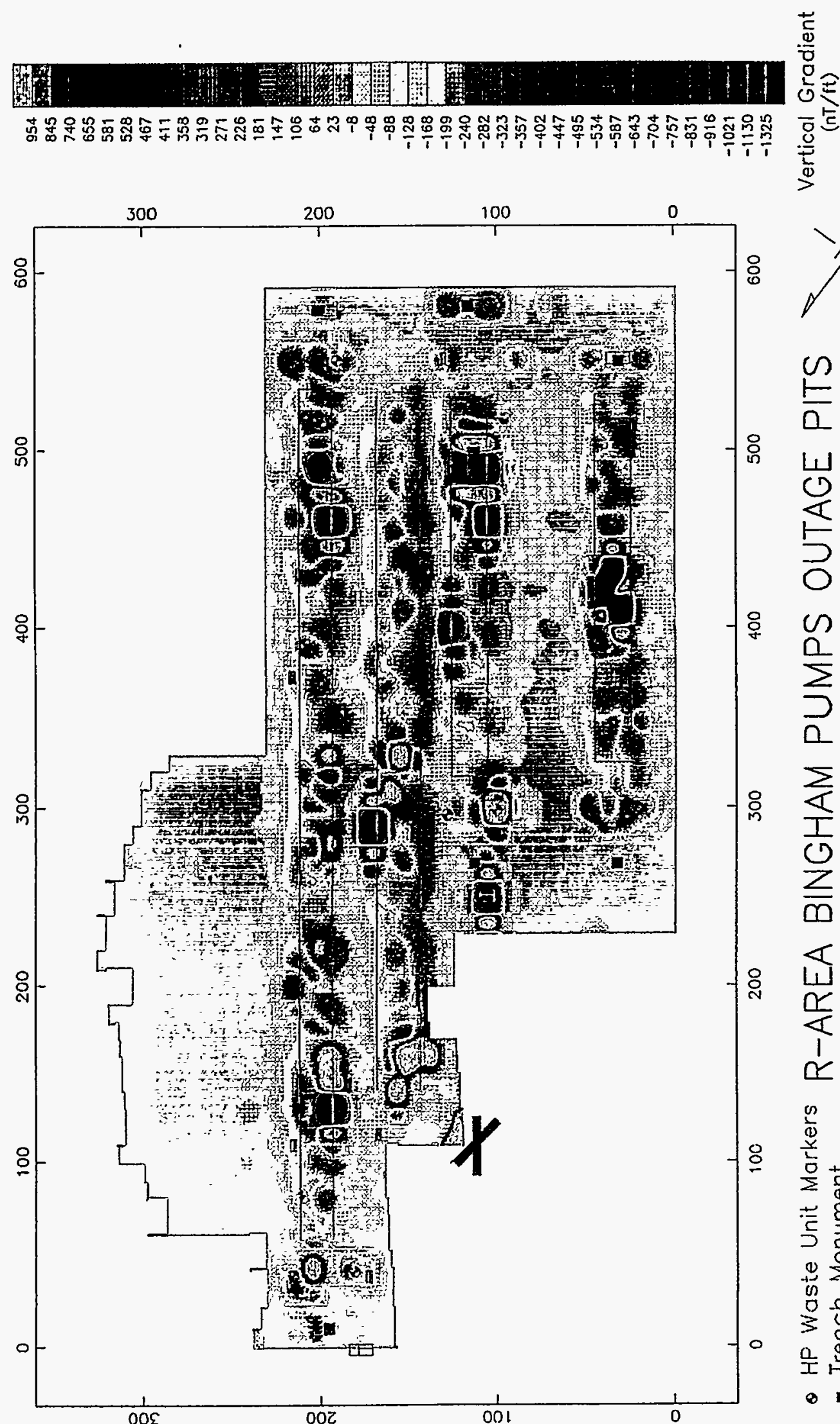

8

$>$

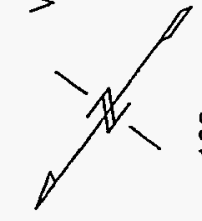

일

$\frac{v}{a}$

in

4

造

in

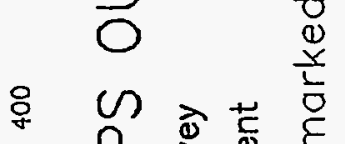

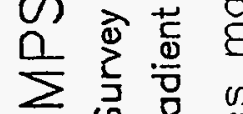

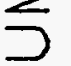

约 $\frac{0}{0}$

웜

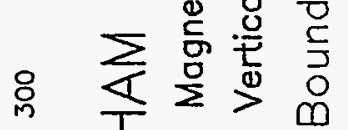

$\frac{1}{3}$

:

L

灾

总

ีำ

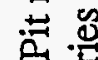

品 超

吾官

를 뎡

A.

曷

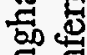

$. \Xi . \Xi$

品

Ð

\&

चु

忌 苋

$\frac{\pi}{\alpha}$

8 궁

응 密

8 㐫

$+\varepsilon$ n

ऽ 능 $\overline{0}$

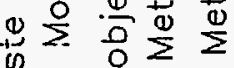

它

$\circ$

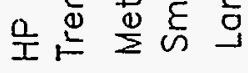

- I. 目 
This page intentionally left blank. 


\section{Appendix A-R-Area Bingham Pump Outage Pits Magnetic Survey Data}





\begin{tabular}{|c|c|c|c|c|c|c|c|c|c|c|c|c|}
\hline & & & & & & & $S P(n T)$ & & BOT & TOM (nT) & & \\
\hline Line & Day & Time & Sta. & $x$ & $Y$ & uncorr. & corr. & diff. & uncorr. & corr. & diff. & \\
\hline 0 & 361 & 134114 & 10 & 590 & 0 & 51495.6 & 51495.6 & 0.0 & 51496.2 & 51496.2 & 0.0 & 0.3 \\
\hline 0 & 361 & 134125 & 12 & 590 & 5 & 51502.7 & 51502.7 & 0.0 & 51499.0 & 51499.0 & 0.0 & -1.9 \\
\hline 0 & 361 & 134135 & 14 & 590 & 10 & 51504.6 & 51504.6 & 0.0 & 51501.0 & 51501.1 & -0.1 & -1.8 \\
\hline 0 & 361 & 134145 & 16 & 590 & 15 & 51495.1 & 51495.1 & 0.0 & 51495.4 & 51495.5 & -0.1 & 0.2 \\
\hline 0 & 361 & 134154 & 18 & 590 & 20 & 51500.9 & 51500.9 & 0.0 & 51494.1 & 51494.2 & -0.1 & -3.4 \\
\hline 0 & 361 & 134204 & 20 & 590 & 25 & 51494.9 & 51494.9 & 0.0 & 51498.9 & 51499.0 & -0.1 & 2.0 \\
\hline 0 & 361 & 134214 & 22 & 590 & 30 & 51494.4 & 51494.5 & -0.1 & 51490.3 & 51490.4 & -0.1 & -2.1 \\
\hline 0 & 361 & 134223 & 24 & 590 & 35 & 51489.7 & 51489.8 & -0.1 & 51490.5 & 51490.6 & -0.1 & 0.4 \\
\hline 0 & 361 & 134233 & 26 & 590 & 40 & 51488.0 & 51488.1 & -0.1 & 51487.5 & 51487.6 & -0.1 & -0.3 \\
\hline 0 & 361 & 134243 & 28 & 590 & 45 & 51488.4 & 51488.5 & -0.1 & 51487.1 & 51487.2 & -0.1 & -0.7 \\
\hline 0 & 361 & 134253 & 30 & 590 & 50 & 51483.1 & 51483.2 & -0.1 & 51483.4 & 51483.5 & -0.1 & 0.2 \\
\hline 0 & 361 & 134304 & 32 & 590 & 55 & 51467.5 & 51467.6 & -0.1 & 51463.9 & 51464.0 & -0.1 & -1.8 \\
\hline 0 & 361 & .134315 & 34 & 590 & 60 & 51427.8 & 51427.9 & -0.1 & 51386.5 & 51386.7 & -0.2 & -20.7 \\
\hline 0 & 361 & 134326 & 36 & 590 & 65 & 51464.4 & 51464.5 & -0.1 & 51472.1 & 51472.3 & -0.2 & 3.9 \\
\hline 0 & 361 & 134337 & 38 & 590 & 70 & 51483.2 & 51483.3 & -0.1 & 51428.8 & 51429.0 & -0.2 & -27.2 \\
\hline 0 & 361 & 134347 & 40 & 590 & 75 & 51489.3 & 51489.4 & -0.1 & 51482.2 & 51482.4 & -0.2 & -3.6 \\
\hline 0 & 361 & 134357 & 42 & 590 & 80 & 51491.0 & 51491.1 & -0.1 & 51482.6 & 51482.8 & -0.2 & -4.2 \\
\hline 0 & 361 & 134408 & 44 & 590 & 85 & 51494.5 & 51494.6 & -0.1 & 51486.6 & 51486.8 & -0.2 & -4.0 \\
\hline 0 & 361 & 134419 & 46 & 590 & 90 & 51490.3 & 51490.4 & -0.1 & 51483.5 & 51483.7 & -0.2 & -3.4 \\
\hline 0 & 361. & 134430 & 48 & 590 & 95 & 51482.5 & 51482.6 & -0.1 & 51476.5 & 51476.7 & -0.2 & -3.0 \\
\hline 0 & 361 & 134441 & 50 & 590 & 100 & 51467.1 & 51467.2 & -0.1 & 51414.9 & 51415.1 & -0.2 & -26.1 \\
\hline 0 & 361 & 134451 & 52 & 590 & 105 & 51463.9 & 51464.0 & -0.1 & 51373.4 & 51373.7 & -0.3 & -45.3 \\
\hline 0 & 361 & 134504 & 54 & 590 & 110 & 51476.2 & 51476.4 & -0.2 & 51342.2 & 51342.5 & -0.3 & -67.0 \\
\hline 0 & 361 & 134514 & 56 & 590 & 115 & 51481.8 & 51482.0 & -0.2 & 51362.2 & 51362.5 & -0.3 & -59.8 \\
\hline 0 & 361 & 134525 & 58 & 590 & 120 & 51465.8 & 51466.0 & -0.2 & 51402.3 & 51402.6 & -0.3 & -31.8 \\
\hline 0 & 361 & 134536 & 60 & 590 & 125 & 51472.8 & 51473.0 & -0.2 & 51418.7 & 51419.0 & -0.3 & -27.1 \\
\hline 0 & 361 & 134549 & 62 & 590 & 130 & 51471.3 & 51471.5 & -0.2 & 51422.3 & 51422.6 & -0.3 & -24.5 \\
\hline 0 & 361 & 134600 & 64 & 590 & 135 & 51473.1 & 51473.3 & -0.2 & 51444.4 & 51444.7 & -0.3 & -14.4 \\
\hline 0 & 361 & 134611 & 66 & 590 & 140 & 51480.7 & 51480.9 & -0.2 & 51470.4 & 51470.7 & -0.3 & -5.2 \\
\hline 0 & 361 & 134622 & 68 & 590 & 145 & 51494.9 & 51495.1 & -0.2 & 51500.4 & 51500.7 & -0.3 & 2.8 \\
\hline 0 & 361 & 134633 & 70 & 590 & 150 & 51503.5 & 51503.7 & -0.2 & 51508.5 & 51508.9 & -0.4 & 2.5 \\
\hline 0 & 361 & 134644 & 72 & 590 & 155 & 51507.3 & 51507.5 & -0.2 & 51520.9 & 51521.3 & -0.4 & 6.8 \\
\hline 0 & 361 & 134655 & 74 & 590 & 160 & 51511.9 & 51512.1 & -0.2 & 51523.0 & 51523.4 & -0.4 & 5.6 \\
\hline 0 & 361. & 134705 & 76 & 590 & 165 & 51517.3 & 51517.5 & -0.2 & 51524.2 & 51524.6 & -0.4 & 3.5 \\
\hline 0 & 361 & 134716 & 78 & 590 & 170 & 51524.2 & 51524.4 & -0.2 & 51528.3 & 51528.7 & -0.4 & 2.1 \\
\hline 0 & 361 & 134725 & 80 & 590 & 175 & 51536.0 & 51536.2 & -0.2 & 51531.7 & 51532.1 & -0.4 & -2.2 \\
\hline 0 & 361 & 134735 & 82 & 590 & 180 & 51542.0 & 51542.2 & -0.2 & 51537.4 & 51537.8 & -0.4 & -2.3 \\
\hline 0 & 361 & 134745 & 84 & 590 & 185 & .51543 .5 & 51543.8 & -0.3 & 51532.0 & 51532.4 & -0.4 & -5.8 \\
\hline 0 & 361 & 134755 & 86 & 590 & 190 & 51540.3 & 51540.6 & -0.3 & 51529.1 & 51529.5 & -0.4 & -5.6 \\
\hline 0 & 361 & 134804 & 88 & 590 & 195 & 51535.1 & 51535.4 & -0.3 & 51523.6 & 51524.1 & -0.5 & -5.8 \\
\hline 0 & 361 & 134814 & 90 & 590 & 200 & 51526.8 & 51527.1 & -0.3 & 51510.5 & 51511.0 & -0.5 & -8.2 \\
\hline 0 & 361 & 134824 & 92 & 590 & 205 & 51520.0 & 51520.3 & -0.3 & 51516.6 & 51517.1 & -0.5 & -1.7 \\
\hline 0 & 361 & 134839 & 94 & 590 & 210 & 51521.1 & 51521.4 & -0.3 & 51505.4 & 51505.9 & -0.5 & -7.9 \\
\hline 0 & 361 & 134848 & 96 & 590 & 215 & 51502.1 & 51502.4 & -0.3 & 51497.9 & 51498.4 & -0.5 & -2.1 \\
\hline 0 & 361 & 134859 & 98 & 590 & 220 & 51486.2 & 51486.5 & -0.3 & 51495.6 & 51496.1 & -0.5 & 4.7 \\
\hline 0 & 361 & 134910 & 100 & 590 & 225 & 51480.2 & 51480.5 & -0.3 & 51492.3 & 51492.8 & -0.5 & 6.1 \\
\hline 0 & 361 & 134921 & 102 & 590 & 230 & 51489.1 & $|51489.4|$ & $=0.3$ & 51521.7 & 51522.2 & -0.5 & 16.3 \\
\hline
\end{tabular}




\begin{tabular}{|c|c|c|c|c|c|c|c|c|c|c|c|c|}
\hline \multirow{2}{*}{ Line } & \multirow[b]{2}{*}{ Day } & \multirow[b]{2}{*}{ Time } & \multirow[b]{2}{*}{ Sta: } & \multirow[b]{2}{*}{$x$} & \multirow[b]{2}{*}{$\mathbf{Y}$} & \multicolumn{3}{|c|}{ TOP (nT) } & \multicolumn{2}{|c|}{ BOTTOM (nT) } & \multirow[b]{2}{*}{ diff. } & \multirow{2}{*}{$\begin{array}{c}\text { Vert.Grad. } \\
\text { (nT/ft) }\end{array}$} \\
\hline & & & & & & uncorr. & corr. & diff. & uncorr. & corr. & & \\
\hline 1 & 361 & 135927 & 104 & 580 & 230 & 51459.5 & 51460.2 & -0.7 & 51476.4 & 51477.6 & -1.2 & 8.5 \\
\hline 1 & 361 & 135937 & 106 & 580 & 225 & 51479.6 & 51480.3 & -0.7 & 51488.4 & 51489.6 & -1.2 & 4.4 \\
\hline 1 & 361 & 135948 & 108 & 580 & 220 & 51512.3 & 51513.0 & -0.7 & 51494.4 & 51495.6 & -1.2 & -9.0 \\
\hline 1 & 361 & 140000 & 110 & 580 & 215 & $5 \cdot 1524.0$ & 51524.7 & -0.7 & 51520.1 & 51521.3 & -1.2 & -2.0 \\
\hline 1 & 361 & 140010 & 112 & 580 & 210 & $5 \cdot 1551.6$ & 51552.3 & -0.7 & 51534.9 & 51536.1 & -1.2 & -8.4 \\
\hline 1 & 361 & 140019 & 114 & 580 & 205 & 51597.9 & 51598.6 & -0.7 & 51552.9 & 51554.1 & -1.2 & -22.5 \\
\hline 1 & 361 & 140030 & 116 & 580 & 200 & 51506.9 & 51507.6 & -0.7 & 51754.1 & 51755.3 & -1.2 & 123.6 \\
\hline 1 & 361 & 140042 & 118 & 580 & 195 & 51927.2 & 51927.9 & -0.7 & $\bullet$ & * & * & * \\
\hline 1 & 361 & 140053 & 120 & 580 & 190 & 51570.2 & 51570.9 & -0.7 & 51546.9 & 51548.1 & -1.2 & -11.7 \\
\hline 1 & 361 & 140104 & 122 & 580 & 185 & 51605.2 & 51605.9 & -0.7 & 51606.8 & 51608.1 & -1.3 & 0.8 \\
\hline 1 & 361 & 140115 & 124 & 580 & 180 & 51603.8 & 51604.5 & -0.7 & 51592.3 & 51593.6 & -1.3 & -5.8 \\
\hline 1 & 361 & 140126 & 126 & 580 & 175 & 51590.9 & 51591.7 & -0.8 & 51581.5 & 51582.8 & -1.3 & -4.7 \\
\hline 1 & 361 & 140136 & 128 & 580 & 170 & 51575.6 & 51576.4 & -0.8 & 51571.6 & 51572.9 & -1.3 & -2.0 \\
\hline 1 & 361 & 140146 & 130 & 580 & 165 & 51558.7 & 51559.5 & -0.8 & 51565.9 & 51567.2 & -1.3 & 3.6 \\
\hline 1 & 361 & 140156 & 132 & 580 & 160 & 51544.9 & 51545.7 & -0.8 & 51543.9 & 51545.2 & -1.3 & -0.5 \\
\hline 1 & 361 & 140206 & 134 & 580 & 155 & 51532.6 & 51533.4 & -0.8 & 51529.0 & 51530.3 & -1.3 & -1.8 \\
\hline 1 & 361 & 140216 & 136 & 580 & 150 & 51517.0 & 51517.8 & -0.8 & 51524.8 & 51526.1 & -1.3 & 3.9 \\
\hline 1 & 361 & 140227 & 138 & 580 & 145 & 51507.7 & 51508.5 & -0.8 & 51514.1 & 51515.4 & -1.3 & 3.2 \\
\hline 1 & 361 & 140237 & 140 & 580 & 140 & 51474.0 & 51474.8 & $=0.8$ & 51478.5 & 51479.9 & -1.4 & 2.3 \\
\hline 1 & 361 & 140247 & 142 & 580 & 135 & 51336.3 & 51337.1 & -0.8 & 51250.2 & 51251.6 & -1.4 & -43.1 \\
\hline 1 & 361 & 140257 & 144 & 580 & 130 & 51102.0 & 51102.8 & -0.8 & + & * & & $\star$ \\
\hline 1 & 361 & 140308 & 146 & 580 & 125 & 51601.9 & 51602.7 & -0.8 & .50349 .0 & 50350.4 & -1.4 & -626.5 \\
\hline 1 & 361 & 140319 & 148 & 580 & 120 & 51502.5 & 51503.3 & -0.8 & 51120.1 & 51121.5 & -1.4 & -191.2 \\
\hline 1 & 361 & 140329 & 150 & 580 & 115 & 51810.2 & 51811.0 & -0.8 & $\star$ & 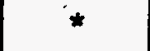 & $\star$ & * \\
\hline 1 & 361 & 140339 & 152 & 580 & 110 & 51323.8 & 51324.6 & -0.8 & 50535.2 & 50536.6 & -1.4 & -394.3 \\
\hline 1 & 361 & 140350 & 154 & 580 & 105 & + & & & & & & * \\
\hline 1 & 361 & 140401 & 156 & 580 & 100 & 51829.2 & 51830.0 & -0.8 & 50008.9 & 50010.3 & -1.4 & -910.2 \\
\hline 1 & 361 & 140412 & 158 & 580 & 95 & 51457.2 & 51458.1 & -0.9 & 51324.6 & 51326.0 & -1.4 & -66.3 \\
\hline 1 & 361 & 140427 & 160 & 580 & 90 & 51491.3 & 51492.2 & -0.9 & 51469.6 & 51471.1 & -1.5 & -10.9 \\
\hline 1 & 361 & 140439 & 162 & 580 & 85 & 51506.8 & 51507.7 & -0.9 & 51493.8 & 51495.3 & -1.5 & -6.5 \\
\hline 1 & 361 & 140449 & 164 & 580 & 80 & 51493.2 & 51494.1 & $=0.9$ & 51504.7 & 51506.2 & -1.5 & 5.8 \\
\hline 1 & 361 & 140459 & 166 & 580 & 75. & 51458.1 & 51459.0 & -0.9 & 51458.3 & 51459.8 & -1.5 & 0.1 \\
\hline 1 & 361 & 140509 & 168 & $580^{\circ}$ & 70 & 51402.9 & 51403.8 & -0.9 & 51058.8 & 51060.3 & -1.5 & -172.1 \\
\hline 1 & 361 & 140521 & 170 & 580 & 65 & 51576.0 & 51576.9 & -0.9 & 52035.7 & 52037.2 & -1.5 & 229.9 \\
\hline 1 & 361 & 140531 & 172 & 580 & 60 & 51475.2 & 51476.1 & -0.9 & 51438.6 & 51440.1 & -1.5 & -18.3 \\
\hline 1 & 361 & 140542 & 174 & 580 & 55 & 51482.2 & 51483.1 & -0.9 & 51489.3 & 51490.8 & -1.5 & 3.6 \\
\hline 1 & 361 & 140552 & 176 & 580 & 50 & 51483.1 & 51484.0 & -0.9 & 51501.4 & 51503.0 & -1.6 & 9.2 \\
\hline 1 & 361 & 140602 & 178 & 580 & 45 & 51483.8 & 51484.7 & -0.9 & 51497.5 & 51499.1 & -1.6 & 6.9 \\
\hline 1 & 361 & 140612 & 180 & 580 & 40 & 51489.3 & 51490.2 & -0.9 & 51499.8 & 51501.4 & -1.6 & 5.3 \\
\hline 1 & 361 & 140622 & 182 & 580 & 35 & 51495.9 & 51496.8 & -0.9 & 51508.5 & 51510.1 & -1.6 & 6.3 \\
\hline 1 & 361 & 140631 & 184 & 580 & 30 & 51495.1 & 51496.0 & -0.9 & 51509.6 & 51511.2 & -1.6 & 7.3 \\
\hline 1 & 361 & 140643 & 186 & 580 & 25 & 51496.0 & 51496.9 & -0.9 & 51526.0 & 51527.6 & -1.6 & 15.0 \\
\hline 1 & 361 & 140654 & 188 & 580 & 20 & 51492.6 & 51493.6 & -1.0 & 51519.4 & 51521.0 & -1.6 & 13.4 \\
\hline 1 & 361 & 140703 & 190 & 580 & 15 & 51489.6 & 51490.6 & -1.0 & 51506.6 & 51508.2 & -1.6 & 8.5 \\
\hline 1 & 361 & 140714 & 192 & 580 & 10 & 51541.7 & 51542.7 & -1.0 & 51577.1 & 51578.7 & -1.6 & 17.7 \\
\hline 1 & 361 & 140728 & 194 & 580 & 5 & 51603.6 & 51604.6 & -1.0 & 51722.0 & 51723.7 & -1.7 & 59.2 \\
\hline 1 & 361 & 140738 & 196 & 580 & 0 & 51518.6 & 51519.6 & -1.0 & 51527.9 & 51529.6 & -1.7 & 4.7 \\
\hline 2 & 361 & 141453 & 208 & 570 & 0 & 51.494 .4 & 51494.0 & 0.4 & 51506.0 & 51507.8 & -1.8 & 5.8 \\
\hline 2 & 361 & $\mid 141504$ & 210 & 570 & 5 & 51468.2 & 51467.8 & 0.4 & 51447.9 & 51449.8 & -1.9 & -10.2 \\
\hline
\end{tabular}




\begin{tabular}{|c|c|c|c|c|c|c|c|c|c|c|c|c|}
\hline \multirow[b]{2}{*}{ Line } & \multirow[b]{2}{*}{ | Day| } & \multirow[b]{2}{*}{ Time } & \multirow[b]{2}{*}{ Sta. } & \multirow[b]{2}{*}{$x$} & \multirow[b]{2}{*}{$\mathbf{Y}$} & \multicolumn{3}{|c|}{ TOP (nT) } & \multicolumn{2}{|c|}{ BOTTOM (nT) } & \multirow[b]{2}{*}{ diff. } & \multirow{2}{*}{$\begin{array}{c}\text { Vert.Grad. } \\
\text { (nT/ft) }\end{array}$} \\
\hline & & & & & & uncorr. & corr. & diff. & uncorr. & corr. & & \\
\hline 2 & 361 & 141513 & 212 & 570 & 10 & 51445.4 & 51445.0 & 0.4 & 51404.6 & 51406.5 & -1.9 & -20.4 \\
\hline 2 & 361 & 141523 & 214 & 570 & 15 & 51493.7 & 51493.2 & 0.5 & 51504.1 & 51506.0 & -1.9 & 5.2 \\
\hline 2 & 361 & 141533 & 216 & 570 & 20 & 51520.2 & 51519.7 & 0.5 & 51516.9 & 51518.8 & -1.9 & -1.7 \\
\hline 2 & 361 & 141542 & 218 & $570 \mid$ & 25 & 51519.4 & 51518.9 & 0.5 & 51500.6 & 51502.5 & -1.9 & -9.4 \\
\hline 2 & 361 . & 141552 & 220 & 570 & 30 & 51511.5 & 51510.9 & 0.6 & 51493.7 & $|51495.6|$ & -1.9 & -8.9 \\
\hline 2 & 361 & 141603 & 222 & 570 & 35 & 51506.0 & 51505.4 & 0.6 & 51485.4 & 51487.3 & -1.9 & -10.3 \\
\hline 2 & 361 & 141612 & 224 & 570 & 40 & 51493.1 & 51492.5 & 0.6 & 51478.1 & 51480.0 & -1.9 & -7.5 \\
\hline 2 & 361 & 141621 & 226 & $|570|$ & 45 & 51492.1 & 51491.4 & 0.7 & 51472.8 & 51474.7 & -1.9 & -9.7 \\
\hline 2 & 361 & 141631 & 228 & 570 & 50 & 51488.2 & 51487.5 & 0.7 & 51469.8 & 51471.7 & -1.9 & -9.2 \\
\hline 2 & 361 & 141640 & 230 & 570 & 55 & 51483.5 & 51482.8 & 0.7 & 51470.8 & 51472.7 & -1.9 & -6.4 \\
\hline 2 & 361 & 141650 & 232 & 570 & 60 & 51488.6 & 51487.8 & 0.8 & 51474.2 & 51476.1 & -1.9 & -7.2 \\
\hline 2 & 361 & 141659 & 234 & 570 & 65 & 51488.4 & 51487.6 & 0.8 & 51477.2 & 51479.1 & -1.9 & -5.6 \\
\hline 2 & 361 & 141709 & 236 & 570 & 70 & 51489.0 & 51488.2 & 0.8 & 51479.8 & 51481.7 & -1.9 & -4.6 \\
\hline 2 & 361 & 141720 & 238 & 570 & 75 & 51498.1 & 51497.2 & 0.9 & 51499.0 & 51500.9 & -1.9 & 0.5 \\
\hline 2 & 361 & 141729 & 240 & 570 & 80 & 51516.1 & 51515.2 & 0.9 & 51507.2 & 51509.1 & -1.9 & -4.5 \\
\hline 2 & 361 & 141739 & 242 & 570 & 85 & 51534.3 & 51533.4 & 0.9 & 51514.8 & 51516.7 & -1.9 & -9.8 \\
\hline 2 & 361 & 141748 & 244 & 570 & 90 & 51543.7 & 51542.7 & 1.0 & 51522.4 & 51524.3 & -1.9 & -10.7 \\
\hline 2 & 361 & 141758 & 246 & 570 & 95 & 51554.7 & 51553.7 & 1.0 & 51521.2 & 51523.1 & -1.9 & -16.8 \\
\hline 2 & 361 & 141808 & 248 & 570 & 100 & 51546.6 & 51545.6 & 1.0 & 51507.3 & 51509.2 & -1.9 & -19.7 \\
\hline 2 & 361 & 141818 & 250 & $570 \mid$ & 105 & .51545 .8 & 51544.7 & 1.1 & 51500.5 & 51502.4 & -1.9 & -22.7 \\
\hline 2 & 361 & 141828 & 252 & $570 \mid$ & 110 & 51618.2 & 51617.1 & 1.1 & 51623.9 & 51625.8 & -1.9 & 2.9 \\
\hline 2 & 361 & 141838 & 254 & 570 & 115 & 51668.2 & 51667.1 & 1.1 & 51656.0 & $|51657.9|$ & -1.9 & -6.1 \\
\hline 2 & 361 & 141848 & 256 & 570 & 120 & 51663.0 & 51661.8 & 1.2 & 51646.8 & 51648.7 & -1.9 & -8.1 \\
\hline 2 & 361 & 141857 & 258 & 570 & 125 & 51598.2 & 51597.0 & 1.2 & 51584.9 & 51586.8 & -1.9 & -6.7 \\
\hline 2 & $361^{\circ}$ & 141907 & 260 & 570 & 130 & 51521.4 & 51520.2 & 1.2 & 51524.0 & 51525.9 & -1.9 & 1.3 \\
\hline 2 & 361 & 141918 & 262 & $570 \mid$ & 135 & 51504.0 & 51502.7 & 1.3 & 51565.4 & 51567.3 & -1.9 & 30.7 \\
\hline 2 & 361 & 141929 & 264 & 570 & 140 & 51498.6 & 51497.3 & 1.3 & 51426.6 & 51428.6 & -2.0 & -36.0 \\
\hline 2 & 361 & 141940 & 266 & $\mid 570$ & 145 & 51524.5 & 51523.2 & 1.3 & 51524.9 & |51526.9 & -2.0 & 0.2 \\
\hline 2 & 361 & 141951 & 268 & 570 & 150 & 51528.4 & 51527.0 & 1.4 & 51524.9 & 51526.9 & -2.0 & -1.8 \\
\hline 2 & 361 & 142001 & 270 & 570 & 155 & 51544.5 & 51543.1 & 1.4 & 51527.6 & 51529.6 & -2.0 & -8.5 \\
\hline 2 & 361 & 142011 & 272 & 570 & 160 & 51566.3 & 51564.9 & 1.4 & 51554.4 & 51556.4 & -2.0 & -6.0 \\
\hline 2 & 361 & 142020 & 274 & 570 & 165 & 51596.6 & 51595.1 & 1.5 & 51570.2 & 51572.2 & -2.0 & -13.2 \\
\hline 2 & 361 & 142030 & 276 & 570 & 170 & 51629.2 & 51627.7 & 1.5 & 51600.6 & $\mid 51602.6$ & -2.0 & -14.3 \\
\hline 2 & 361 & 142040 & 278 & 570 & 175 & 51675.3 & 51673.8 & 1.5 & 51646.2 & 51648.2 & -2.0 & -14.6 \\
\hline 2 & 361 & 142053 & 280 & 570 & 180 & 51736.8 & 51735.2 & 1.6 & 51695.9 & 51697.9 & -2.0 & -20.5 \\
\hline 2 & 361 & 142104 & 282 & 570 & 185 & 51764.3 & 51762.7 & 1.6 & 51713.9 & 51715.9 & -2.0 & -25.2 \\
\hline 2 & 361 & 142114 & 284 & 570 & 190 & 51787.2 & 51785.5 & 1.7 & 51739.8 & 51741.8 & -2.0 & -23.7 \\
\hline 2 & 361 & 142124 & 286 & 570 & 195 & 51787.1 & 51785.4 & 1.7 & 51746.3 & 51748.3 & -2.0 & -20.4 \\
\hline 2 & 361 & 142135 & 288 & 570 & 200 & 51759.7 & 51758.0 & 1.7 & 51716.9 & 51718.9 & -2.0 & -21.4 \\
\hline 2 & 361 & 142145 & 290 & 570 & 205 & 51734.1 & 51732.3 & 1.8 & 51669.0 & 51671.0 & -2.0 & -32.6 \\
\hline 2 & 361 & 155 & 292 & 570 & 210 & & 69.3 & 1.8 & 17.9 & 51619.9 & -2.0 & -26.6 \\
\hline 2 & 361 & 142205 & 294 & 570 & 215 & 6.7 & 04.9 & 1.8 & 51562.8 & 51564.8 & -2.0 & -22.0 \\
\hline 2 & 361 & 142215 & 296 & 570 & 220 & 51538.9 & 51537.0 & 1.9 & 51504.1 & 51506.1 & -2.0 & -17.4 \\
\hline 2 & 361 & 142226 & 298 & 570 & 225 & 51499.3 & 51497.4 & 1.9 & 51463.6 & 51465.6 & -2.0 & -17.9 \\
\hline 2 & 361 & 142236 & 300 & 570 & 230 & 51475.0 & 51473.1 & 1.9 & 51451.8 & 51453.8 & -2.0 & -11.6 \\
\hline 3 & 361 & 142611 & 302 & 560 & 230 & 51427.5 & 51424.8 & 2.7 & 51383.6 & 51385.7 & -2.1 & -22.0 \\
\hline 3 & 361 & 142623 & 304 & 560 & 225 & 51433.4 & 51430.7 & 2.7 & 51366.7 & 51368.8 & -2.1 & -33.4 \\
\hline 3 & 361 & 142634 & 306 & 560 & 220 & 51501.7 & 51498.9 & 2.8 & 51363.1 & $|51365.2|$ & -2.1 & -69.3 \\
\hline$\rightarrow$ & 361 & 142646 & 308 & 560 & 215 & 51732.4 & 51728.6 & 2.8 & 51482.8 & $|51484.9|$ & -2.1 & -124.8 \\
\hline
\end{tabular}




\begin{tabular}{|c|c|c|c|c|c|c|c|c|c|c|c|c|}
\hline Line & Day & Time & Sta. & $x$ & $\mathbf{Y}$ & uncorr. & $\begin{array}{l}\text { (nT) } \\
\text { corr. }\end{array}$ & diff. & $\begin{array}{l}\text { BO1 } \\
\text { uncorr. }\end{array}$ & $\begin{array}{l}\text { TOM (nT } \\
\text { corr. }\end{array}$ & diff. & $\begin{array}{c}\text { Vert.Grad. } \\
\text { (nT/ft) }\end{array}$ \\
\hline 3 & 361 & 142657 & 310 & 560 & 210 & 51947.0 & 51944.2 & 2.8 & 51755.4 & 51757.5 & -2.1 & -95.8 \\
\hline 3 & 361 & 142708 & 312 & 560 & 205 & 52182.1 & 52179.2 & 2.9 & 52046.2 & 52048.3 & -2.1 & -68.0 \\
\hline 3 & 361 & 142719 & 314 & $|560|$ & 200 & 52347.2 & 52344.3 & 2.9 & 52118.3 & 52120.4 & -2.1 & -114.5 \\
\hline 3 & 361 & 142729 & 316 & 560 & 195 & 52464.7 & 52461.8 & 2.9 & 52519.4 & 52521.5 & -2.1 & 27.4 \\
\hline 3 & 361 & 142740 & 318 & 560 & 190 & 52298.7 & 52295.7 & 3.0 & 52149.0 & 52151.1 & -2.1 & -74.9 \\
\hline 3 & 361 & 142750 & 320 & 560 & 185 & 52072.8 & 52069.8 & 3.0 & 51757.4 & 51759.5 & -2.1 & -157.7 \\
\hline 3 & 361 & 142801 & 322 & 560 & 180 & 52032.7 & 52029.6 & 3.1 & 52352.0 & 52354.1 & -2.1 & 159.7 \\
\hline 3 & 361 & 142811 & 324 & 560 & 175 & 51829.7 & 51826.6 & 3.1 & 51793.0 & 51795.1 & -2.1 & -18.4 \\
\hline 3 & 361 & 142821 & 326 & 560 & 170 & 51706.5 & 51703.4 & 3.1 & 51669.8 & 51671.9 & -2.1 & -18.4 \\
\hline 3 & 361 & 142831 & 328 & 560 & 165 & 51635.4 & 51632.2 & 3.2 & 51607.7 & 51609.9 & -2.2 & -13.9 \\
\hline 3 & 361 & 142841 & 330 & 560 & 160 & 51592.2 & 51589.0 & 3.2 & 51563.2 & 51565.4 & -2.2 & -14.5 \\
\hline 3 & 361 & 142851 & 332 & 560 & 155 & 51568.6 & 51565.4 & 3.2 & 51546.2 & 548.4 & -2.2 & -11.2 \\
\hline 3 & 361 & 142901 & 334 & $560 \mid$ & 150 & 51550.3 & 51547.0 & 3.3 & 51524.5 & 51526.7 & -2.2 & -12.9 \\
\hline 3 & 361 & 142910 & .336 & 560 & 145 & 51533.0 & 51529.7 & 3.3 & 51501.2 & 51503.4 & -2.2 & -15.9 \\
\hline 3 & 361 & 142920 & 338 & 560 & 140 & 51523.6 & 51520.3 & 3.3 & 51488.9 & 51491.1 & -2.2 & -17.4 \\
\hline 3 & 361 & 142930 & 340 & $560 \mid$ & 135 & 51553.4 & 51550.0 & 3.4 & 51504.4 & 51506.6 & -2.2 & -24.5 \\
\hline 3 & 361 & 142941 & 342 & 560 & 130 & 51756.8 & 51753.4 & 3.4 & 51930.7 & 51932.9 & -2.2 & 87.0 \\
\hline 3 & 361 & 143006 & 344 & 560 & 125 & 51943.8 & 51940.3 & 3.5 & 52115.0 & 52117.2 & -2.2 & 85.6 \\
\hline 3 & 361 & 143017 & 346 & 560 & 120 & 52075.9 & 52072.4 & 3.5 & 52244.2 & 52246.4 & -2.2 & 84.2 \\
\hline 3 & 361 & 143027 & 348 & 560 & 115 & 52173.5 & 52169.9 & 3.6 & 52350.5 & 52352.7 & -2.2 & 88.5 \\
\hline 3 & 361 & 143038 & 350 & 560 & 110 & 52240.6 & 52237.0 & 3.6 & 52455.0 & 52457.2 & -2.2 & 107.2 \\
\hline 3 & 361 & 143049 & 352 & $|560|$ & 105 & 52146.9 & 52143.3 & 3.6 & 52366.8 & 52369.0 & -2.2 & 110.0 \\
\hline 3 & 361 & 143100 & 354 & $|560|$ & 100 & 51811.0 & 51807.3 & 3.7 & 51808.3 & 51810.5 & -2.2 & -1.4 \\
\hline 3 & 361 & 143110 & 356 & $|560|$ & 95 & 51650.4 & 51646.7 & 3.7 & 51615.7 & 51617.9 & -2.2 & -17.4 \\
\hline 3 & 361 & 143120 & 358 & 560 & 90 & 51586.9 & 51583.2 & 3.7 & 51544.5 & 51546.7 & -2.2 & -21.2 \\
\hline 3 & 361 & 143129 & 360 & $560 \mid$ & 85 & 51550.7 & 51546.9 & 3.8 & 51525.3 & 51527.5 & -2.2 & -12.7 \\
\hline 3 & 361 & 143139 & 362 & 560 & 80 & 51527.3 & 51523.5 & 3.8 & 51515.4 & 51517.6 & -2.2 & -6.0 \\
\hline 3 & 361 & 143149 & 364 & 560 & 75 & 51506.1 & 51502.3 & 3.8 & 51511.2 & 51513.4 & -2.2 & 2.6 \\
\hline 3 & 361 & 143159 & 366 & 560 & 70 & 51492.4 & 51488.5 & 3.9 & 51493.4 & 51495.6 & -2.2 & 0.5 \\
\hline 3 & 361 & 143209 & 368 & $560 \mid$ & 65 & 51478.4 & 5.1474 .5 & 3.9 & 51476.3 & 51478.5 & -2.2 & -1.1 \\
\hline 3 & 361 & 143218 & 370 & $560 \mid$ & 60 & 51459.2 & 51455.3 & 3.9 & 51459.6 & 51461.8 & -2.2 & 0.2 \\
\hline 3 & 361 & 143228 & 372 & 560 & 55 & 51454.6 & 51450.6 & 4.0 & 51423.8 & 51426.0 & -2.2 & -15.4 \\
\hline 3 & 361 & 143238 & 374 & $560 \mid$ & 50 & 51472.2 & 51468.2 & 4.0 & 51396.3 & 51398.5 & -2.2 & -38.0 \\
\hline 3 & 361 & 143248 & 376 & 560 & 45 & 51513.5 & 51509.5 & 4.0 & 51416.9 & 51419.1 & -2.2 & -48.3 \\
\hline 3 & 361 & 143259 & 378 & 560 & 40 & 51522.9 & 51518.8 & 4.1 & 51451.1 & 514 & -2.3 & -35.9 \\
\hline 3 & 361 & 143309 & 380 & $560 \mid$ & 35 & 51536.2 & 51532.1 & 4.1 & 51462.0 & 51464.3 & -2.3 & -37.1 \\
\hline 3 & 361 & 143319 & 382 & $560 \mid$ & 30 & 51563.4 & 51559.3 & 4.1 & 51469.1 & 51471.4 & -2.3 & -47.2 \\
\hline 3 & 361 & 143331 & 384 & 560 & 25 & 51580.9 & 51576.7 & 4.2 & 51482.9 & 51485.2 & -2.3 & -49.0 \\
\hline 3 & 361 & 143341 & 386 & $560 \mid$ & 20 & 51571.2 & 51567.0 & 4.2 & 51463.5 & 51465.8 & -2.3 & -53.9 \\
\hline 3 & 361 & 143352 & 388 & $|560|$ & 15 & 51554.5 & 51550.2 & 4.3 & 51495.9 & 51498.2 & -2.3 & -29.3 \\
\hline 3 & 361 & 143402 & 390 & $|560|$ & 10 & 51549.2 & 51544.9 & 4.3 & 51532.1 & 51534.4 & -2.3 & -8.6 \\
\hline 3 & 361 & 143413 & 392 & $560 \mid$ & 5 & 51532.8 & 51528.5 & 4.3 & 51535.9 & 51538.2 & -2.3 & 1.6 \\
\hline 3 & 361 & 143422 & 394 & $560 \mid$ & 0 & 51525.8 & 51521.4 & 4.4 & 51525.1 & 51527.4 & -2.3 & -0.4 \\
\hline 4 & 361 & 144429 & 406 & $550 \mid$ & 0 & 51551.8 & 51546.5 & 5.3 & 51542.4 & 51543.0 & -0.6 & -4.7 \\
\hline 4 & 361 & 144441 & 408 & 550 & 5 & 51582.1 & 51576.7 & 5.4 & 51551.1 & 51551.7 & -0.6 & -15.5 \\
\hline 4 & 361 & 144452 & 410 & 550 & 10 & 51612.6 & 51607.2 & 5.4 & 51520.8 & 51521.3 & -0.5 & -45.9 \\
\hline 4 & 361 & 144505 & 412 & $|550|$ & 15 & 51741.3 & 51735.8 & 5.5 & 50868.4 & 50868.9 & -0.5 & -436.5 \\
\hline 4 & 361 & 144518 & 414 & $550 \mid$ & 20 & & 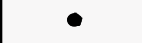 & * & + & & * & * \\
\hline 4 & 361 & 144530 & 416 & $550 \mid$ & 25 & 52306.3 & 52300.8 & 5.5 & 52074.9 & 52075.3 & -0.4 & -115.7 \\
\hline
\end{tabular}




\begin{tabular}{|c|c|c|c|c|c|c|c|c|c|c|c|c|}
\hline Line & Day & Time & Sta. & $\mathbf{x}$ & $\mathbf{Y}$ & uncorr. & $\begin{array}{l}\text { SP (nT) } \\
\text { corr. }\end{array}$ & diff. & $\begin{array}{l}\text { BOT } \\
\text { uncorr. }\end{array}$ & $\begin{array}{l}\text { TOM (nT) } \\
\text { corr. }\end{array}$ & diff. & $\begin{array}{c}\text { Vert.Grad. } \\
\text { (nT/ft) }\end{array}$ \\
\hline 4 & 361 & 144542 & 418 & 550 & 30 & 52116.6 & 52111.1 & 5.5 & 52229.5 & 52229.9 & -0.4 & 56.5 \\
\hline 4 & 361 & 144553 & 420 & 550 & 35 & 51616.4 & 51610.9 & 5.5 & 51308.0 & 51308.4 & -0.4 & -154.2 \\
\hline 4 & 361 & 144603 & 422 & 550 & 40 & 51830.8 & 51825.3 & 5.5 & 51646.1 & 51646.4 & -0.3 & -92.4 \\
\hline 4 & 361 & 144613 & 424 & 550 & 45 & 52872.0 & 52866.4 & 5.6 & 52201.4 & 52201.7 & -0.3 & -335.3 \\
\hline 4 & 361 & 144623 & 426 & 550 & 50 & 51475.7 & 51470.1 & 5.6 & $\star$ & * & $\star$ & - \\
\hline 4 & 361 & 144634 & 428 & 550 & 55 & 51319.6 & 51314.0 & 5.6 & 51170.2 & 51170.4 & -0.2 & -74.7 \\
\hline 4 & 361 & 144644 & 430 & 550 & 60 & 51419.8 & 51414.2 & 5.6 & 51382.8 & 51383.0 & -0.2 & -18.5 \\
\hline 4 & 361 & 144654 & 432 & 550 & 65 & 51458.3 & 51452.7 & 5.6 & 51438.9 & 51439.1 & -0.2 & -9.7 \\
\hline 4 & 361 & 144704 & 434 & 550 & 70 & 51486.1 & 51480.5 & 5.6 & 51471.2 & 51471.3 & -0.1 & -7.5 \\
\hline 4 & 361 & 144714 & 436 & 550 & 75 & 51507.7 & 51502.0 & 5.7 & 51485.8 & 51485.9 & -0.1 & -11.0 \\
\hline 4 & 361 & 144724 & $438^{\circ}$ & 550 & 80 & .51526 .4 & .51520 .7 & 5.7 & 51476.3 & 51476.4 & -0.1 & -25.1 \\
\hline 4 & 361 & 144734 & 440 & 550 & 85 & 51708.2 & 51702.5 & 5.7 & 51087.6 & 51087.6 & 0.0 & -310.3 \\
\hline 4 & 361 & 144744 & 442 & $550^{\circ}$ & 90 & 51849.0 & 51843.3 & 5.7 & $\bullet$ & 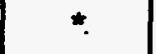 & 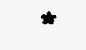 & * \\
\hline 4 & 361 & 144754 & 444 & 550 & 95 & 51624.6 & 51618.9 & 5.7 & 51514.6 & 51514.6 & 0.0 & -55.0 \\
\hline 4 & 361 & 144805 & 446 & 550 & 100 & 51805.4 & 51799.7 & 5.7 & 51708.7 & 1708.7 & 0.0 & -48.4 \\
\hline 4 & 361 & 144815 & 448 & 550 & 105 & 52191.6 & 52185.8 & 5.8 & 52236.6 & 52236.5 & 0.1 & 22.5 \\
\hline 4 & 361 & 144826 & 450 & 550 & 110 & 52694.9 & 52689.1 & 5.8 & * & & & * \\
\hline 4 & 361 & 144837 & 452 & 550 & 115 & 52083.5 & 52077.7 & 5.8 & 51943.6 & 51943.5 & 0.1 & -70.0 \\
\hline 4 & 361 & 144847 & 454 & 550 & 120 & 51973.1 & 51967.3 & 5.8 & 51959.6 & 51959.4 & 0.2 & -6.8 \\
\hline 4 & 361 & 144900 & 456 & 550 & 125 & 52033.2 & 52027.4 & 5.8 & .53326 .4 & 53326.2 & 0.2 & 646.6 \\
\hline 4 & 361 & 144911 & 458 & 550 & 130 & 51750.9 & 51745.1 & 5.8 & 50760.1 & 50759.9 & 0.2 & -495.4 \\
\hline 4 & 361. & 144923 & 460 & 550 & 135 & 51543.2 & 51537.3 & 5.9 & 51391.2 & 51390.9 & 0.3 & -76.0 \\
\hline 4 & 361 & 144933 & 462 & 550 & 140 & 51621.5 & 51615.6 & 5.9 & 51474.3 & 51474.0 & 0.3 & -73.6 \\
\hline 4 & 361 & 1.44944 & 464 & 550 & 145 & 51666.2 & 51660.3 & 5.9 & 51537.5 & 51537.2 & $0.3^{\circ}$ & -64.4 \\
\hline 4 & 361 & 144954 & 466 & 550 & 150 & 51626.9 & 51621.0 & 5.9 & 51546.1 & 51545.7 & 0.4 & -40.4 \\
\hline 4 & 361 & 145004 & 468 & 550 & 155 & 51614.4 & 51608.5 & 5.9 & 51556.2 & 51555.8 & 0.4 & -29.1 \\
\hline 4 & 361 & 145013 & 470 & 550 & 160 & 51623.8 & 51617.9 & 5.9 & 51574.2 & 51573.8 & 0.4 & -24.8 \\
\hline 4 & 361 & 145023 & .472 & 550 & 165 & 51669.9 & 51663.9 & 6.0 & 51604.4 & 51603.9 & 0.5 & -32.8 \\
\hline 4 & 361 & 145031 & 474 & 550 & 170 & 51757.7 & 51751.7 & 6.0 & 51685.5 & $|51685.0|$ & 0.5 & -36.1 \\
\hline 4 & 361 & 145040 & 476 & 550 & 175 & 51910.9 & 51904.9 & 6.0 & 51816.2 & 51815.7 & 0.5 & -47.4 \\
\hline 4 & 361 & 145051 & 478 & 550 & 180 & 52210.6 & 52204.6 & 6.0 & 52012.6 & 52012.1 & 0.5 & -99.0 \\
\hline 4 & 361 & 145102 & 480 & 550 & 185 & 52898.2 & 52892.2 & 6.0 & 51910.9 & 51910.3 & 0.6 & -493.7 \\
\hline 4 & 361 & 145111 & 482 & 550 & 190 & 45499.2 & 45493.2 & 6.0 & • & & 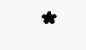 & * \\
\hline 4 & 361 & 145121 & 484 & 550 & 195 & 54118.6 & 54112.5 & 6.1 & 53977.8 & |53977.2 & 0.6 & -70.4 \\
\hline 4 & 361 & 145131 & 486 & 550 & 200 & 55138.5 & 55132.4 & 6.1 & 52043.6 & 52042.9 & 0.7 & -1547.5 \\
\hline 4 & 361 & 145141 & 488 & 550 & 205 & 53964.5 & 53958.4 & 6.1 & 54389.2 & 54388.5 & 0.7 & 212.4 \\
\hline 4 & 361 & 145151 & 490 & 550 & 210 & 52626.5 & 52620.4 & 6.1 & 51659.9 & 51659.2 & 0.7 & -483.3 \\
\hline 4 & 361 & 145201 & 492 & 550 & 215 & 43301.4 & 43295.3 & 6.1 & * & & * & - \\
\hline 4 & 361 & 145212 & 494 & 550 & 220 & 50906.4 & 50900.3 & 6.1 & 49998.2 & 49997.4 & 0.8 & -454.1 \\
\hline 4 & 361 & 145222 & 496 & 550 & 225 & 51217.9 & 51211.8 & 6.1 & .51099 .4 & 51098.6 & 0.8 & $-59,3$ \\
\hline 4 & 361 & 145232 & 498 & 550 & 230 & 51312.7 & 51306.5 & 6.2 & 51258.1 & 51257.2 & 0.9 & -27.3 \\
\hline 5 & 361 & 145641 & 500 & 540 & 230 & 51177.3 & 51170.7 & 6.6 . & 51135.3 & 51133.7 & 1.6 & -21.0 \\
\hline 5 & 361 & 145652 & 502 & 540 & 225 & 51120.3 & 51113.7 & 6.6 & 51049.0 & 51047.4 & 1.6 & -35.7 \\
\hline 5 & 361 & 145708 & 504 & 540 & 220 & 51093.6 & 51087.0 & 6.6 & 50923.0 & 50921.3 & 1.7 & -85.3 \\
\hline 5 & 361 & 145719 & 506 & 540 & 215 & 51290.8 & 51284.2 & 6.6 & 50951.7 & 50950.0 & 1.7 & -169.6 \\
\hline 5 & 361 & 145731 & 508 & 540 & 210 & 52163.8 & 52157.2 & 6.6 & 51593.2 & 51591.4 & 1.8 & -285.3 \\
\hline 5 & 361 & 145742 & 510 & 540 & 205 & 54464.7 & 54458.0 & 6.7 & 54793.3 & 54791.5 & 1.8 & 164.3 \\
\hline 5 & 361 & 145753 & 512 & 540 & 200 & & & 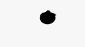 & . &  & - & 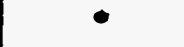 \\
\hline 5 & 361 & $|145804|$ & 514 & 540 & 195 & & 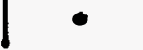 & & 52064.1 & $|52062.2|$ & 1.9 & 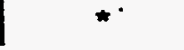 \\
\hline
\end{tabular}




\begin{tabular}{|c|c|c|c|c|c|c|c|c|c|c|c|c|}
\hline & & & & & & & (nT) & & BO7 & - & & 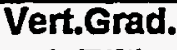 \\
\hline ine & | Day & Time & Sta. & $\mathrm{x}$ & $\mathbf{Y}$ & uncorr. & corr. & diff. & uncorr. & corr. & diff. & \\
\hline 5 & 361 & 145815 & 516 & 540 & 190 & 55651.7 & 55645.0 & 6.7 & 54800.1 & 54798.2 & 1.9 & -425.8 \\
\hline 5 & 361 & 145825 & 518 & 540 & 185 & 53510.6 & 53503.9 & 6.7 & 53668.7 & 53666.8 & 1.9 & 79.1 \\
\hline 5 & 361 & 145836 & 520 & 540 & 180 & 52488.3 & 52481.6 & 6.7 & 52392.6 & 52390.7 & 1.9 & -47.9 \\
\hline 5 & 361 & 145846 & 522 & 540 & 175 & 51945.3 & 51938.5 & 6.8 & 51860.2 & 51858.2 & 2.0 & -42.6 \\
\hline 5 & 361 & 145857 & 524 & 540 & 170 & 51714.4 & 51707.6 & 6.8 & 51644.4 & 51642.4 & 2.0 & -35.0 \\
\hline 5 & 361 & 145907 & 526 & 540 & 165 & 51616.1 & 51609.3 & 6.8 & 51543.8 & 51541.8 & 2.0 & -36.2 \\
\hline 5 & 361 & 145917 & 528 & 540 & 160 & 51659.8 & 51653.0 & 6.8 & 51565.7 & 51563.6 & 2.1 & -47.1 \\
\hline 5 & 361 & 145927 & 530 & 540 & 155 & 51779.1 & 51772.3 & 6.8 & 51755.1 & 51753.0 & 2.1 & -12.0 \\
\hline 5 & 361 & 145938 & 532 & 540 & 150 & 51899.5 & 51892.7 & 6.8 & 51780.0 & 51777.9 & 2.1 & -59.8 \\
\hline 5 & 361 & 145948 & 534 & 540 & 145 & 52197.4 & 52190.5 & 6.9 & 52225.1 & 52222.9 & 2.2 & 13.9 \\
\hline 5 & 361 & 145959 & 536 & 540 & 140 & 52235.4 & 52228.5 & 6.9 & 52582.9 & 52580.7 & 2.2 & 173.8 \\
\hline 5 & 361 & 150009 & 538 & 540 & 135 & 51809.2 & 51802.3 & 6.9 & 51677.3 & 51675.1 & 2.2 & -66.0 \\
\hline 5 & 361 & 150020 & 540 & 540 & 130 & 51614.3 & 51607.4 & 6.9 & 51431.3 & 51429.0 & 2.3 & -91.5 \\
\hline 5 & 361 & 150030 & 542 & 540 & 125 & 51539.5 & 51532.6 & 6.9 & 51147.8 & 51145.5 & 2.3 & -195.9 \\
\hline 5 & 361 & 150041 & 544 & 540 & 120 & 51765.6 & 51758.7 & 6.9 & 51633.7 & 51631.4 & 2.3 & -66.0 \\
\hline 5 & 361 & 150051 & 546 & 540 & 115 & 51970.3 & 51963.3 & 7.0 & 51902.0 & 51899.6 & 2.4 & -34.2 \\
\hline 5 & 361 & 150100 & 548 & 540 & 110 & 51996.4 & 51989.4 & 7.0 & 51939.2 & 51936.8 & 2.4 & -28.6 \\
\hline 5 & 361 & 150111 & 550 & 540 & 105 & 51909.5 & 51902.5 & 7.0 & 51769.3 & 51766.9 & 2.4 & $-70: 1$ \\
\hline 5 & 361 & 150121 & 552 & 540 & 100 & 51808.2 & 51801.2 & 7.0 & 51727.0 & 51724.6 & 2.4 & -40.6 \\
\hline 5 & 361 & 150131 & 554 & 540 & 95 & 51725.4 & 51718.4 & 7.0 & 51680.8 & 51678.3 & 2.5 & -22.3 \\
\hline 5 & 361 & 150140 & 556 & 540 & 90 & 51643.6 & 51636.6 & 7.0 & 51614.5 & 51612.0 & 2.5 & -14.6 \\
\hline 5 & 361 & 150149 & 558 & 540 & 85 & 51589.8 & 51582.8 & 7.0 & 51565.5 & 51563.0 & 2.5 & -12.2 \\
\hline 5 & 361 & 150159 & 560 & 540 & 80 & 51546.0 & 51538.9 & 7.1 & 51527.6 & 51525.0 & 2.6 & -9.2 \\
\hline 5 & 361 & 150208 & 562 & 540 & 75 & 51511.4 & 51504.3 & 7.1 & 51493.5 & 51490.9 & 2.6 & -9.0 \\
\hline 5 & 361 & 150218 & 564 & 540 & 70 & 51471.5 & 51464.4 & 7.1 & 51461.7 & 51459.1 & 2.6 & -4.9 \\
\hline 5 & 361 & 150227 & 566 & 540 & 65 & 51438.0 & 51430.9 & 7.1 & 51423.9 & 51421.3 & 2.6 & -7.1 \\
\hline 5 & 361 & 150236 & 568 & 540 & 60 & 51391.4 & 51384.3 & 7.1 & 51370.5 & 51367.8 & 2.7 & -10.5 \\
\hline 5 & 361 & 150246 & 570 & 540 & 55 & 51337.8 & 51330.7 & 7.1 & 51287.5 & 51284.8 & 2.7 & -25.2 \\
\hline 5 & 361 & 150256 & 572 & 540 & 50 & 51327.2 & 51320.0 & 7.2 & 51185.5 & 51182.8 & 2.7 & -70.9 \\
\hline 5 & 361 & 150307 & 574 & 540 & 45 & 51605.1 & 51597.9 & 7.2 & 51517.0 & 51514.2 & 2.8 & -44.1 \\
\hline 5 & 361 & 150317 & 576 & 540 & 40 & 52116.7 & 52109.5 & 7.2 & 52697.0 & 52694.2 & 2.8 & 290.2 \\
\hline 5 & 361 & 150328 & $\cdot 578$ & 540 & 35 & 52052.5 & 52045.3 & 7.2 & 52134.5 & 52131.7 & 2.8 & 41.0 \\
\hline 5 & 361 & 150338 & 580 & 540 & 30 & 52050.6 & 52043.4 & 7.2 & & $\cdot \star$ & * & « \\
\hline 5 & 361 & 150351 & 582 & 540 & 25 & 52982.4 & 52975.2 & 7.2 & - & - & * & * \\
\hline 5 & 361 & 150401 & 584 & 540 & 20 & 52522.1 & 52514.8 & 7.3 & 52731.5 & 52728.6 & 2.9 & 104.7 \\
\hline 5 & 361 & 150414 & 586 & 540 & 15 & 51964.1 & 51956.8 & 7.3 & 51889.4 & 51886.4 & 3.0 & -37.4 \\
\hline 5 & 361 & 150424 & 588 & 540 & 10 & 51748.1 & 51740.8 & 7.3 & 51701.9 & 51698.9 & 3.0 & -23.1 \\
\hline 5 & 361 & 150435 & 590 & 540 & 5 & 51641.0 & 51633.7 & 7.3 & 51609.2 & 51606.2 & 3.0 & -15.9 \\
\hline 5 & 361 & 150447 & 592 & 540 & 0 & 51581.4 & 51574.1 & 7.3 & 51560.8 & 51557.7 & 3.1 & -10.3 \\
\hline 6 & 361 & 150923 & 604 & 530 & 0 & 51581.1 & 51573.4 & 7.7 & 51566.7 & 51563.4 & 3.3 & -7.2 \\
\hline 6 & 361 & 150935 & 606 & 530 & 5 & 51654.4 & 51646.6 & 7.8 & 51626.9 & 51623.5 & 3.4 & -13.8 \\
\hline 6 & 361 & 150946 & 608 & 530 & 10 & 51788.3 & 51780.5 & 7.8 & 51729.3 & 51725.9 & 3.4 & -29.5 \\
\hline 6 & 361 & 150957 & 610 & 530 & 15 & 52088.6 & 52080.7 & 7.9 & 52029.0 & 52025.6 & 3.4 & -29.8 \\
\hline 6 & 361 & 151008 & 612 & 530 & 20 & 52649.7 & 52641.8 & 7.9 & 52895.1 & 52891.7 & 3.4 & 122.7 \\
\hline 6 & 361 & 151019 & 614 & 530 & 25 & 52981.5 & 52973.6 & 7.9 & 53983.6 & 53980.1 & 3.5 & 501.1 \\
\hline 6 & 361 & 151030 & 616 & 530 & 30 & 52257.6 & 52249.7 & 7.9 & 52181.6 & 52178.1 & 3.5 & -38.0 \\
\hline 6 & 361 & 151040 & 618 & 530 & 35 & 51859.8 & 51851.9 & 7.9 . & 51617.6 & 51614.1 & 3.5 & $-121: 1$ \\
\hline 6 & 361 & 151051 & 620 & 530 & 40 & 51874.1 & 51866.2 & 7.9. & .52356 .2 & 52352.7 & 3.5 & 241.1 \\
\hline 6 & 361 & 151101 & 622 & 530 & 45 & 51351.5 & 51343.5 & 8.0 . & 51013.1 & 51009.6 & 3.5 & -169.2 \\
\hline
\end{tabular}




\begin{tabular}{|c|c|c|c|c|c|c|c|c|c|c|c|c|}
\hline & & mo & & $\mathbf{y}$ & Y & & $\mathrm{SP}(\mathrm{nT})$ & & BOT & TOM (nT) & 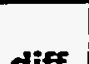 & Vert.Grad. \\
\hline IIE & & & & $\boldsymbol{\Lambda}$ & 1 & & & III. & & & III. & \\
\hline 6 & 361 & 151111 & 624 & 530 & 50 & 51197.4 & 51189.4 & 8.0 & 50972.8 & 50969.3 & 3.5 & -112.3 \\
\hline 6 & 361 & 151121 & 626 & 530 & 55 & 51289.0 & 51281.0 & 8.0 & 51217.9 & 51214.4 & 3.5 & -35.6 \\
\hline 6 & 361 & 151131 & 628 & 530 & 60 & 51365.1 & 51357.1 & 8.0 & 51328.5 & 51325.0 & 3.5 & -18.3 \\
\hline 6 & 361 & 151141 & 630 & 530 & 65 & 51416.4 & 5.1408 .4 & 8.0 & 51391.7 & 51388.2 & 3.5 & -12.4 \\
\hline 6 & 361 & 151150 & 632 & 530 & 70 & 51465.8 & 51457.8 & 8.0 & 51441.1 & 51437.6 & 3.5 & -12.4 \\
\hline 6 & 361 & 151200 & 634 & 530 & 75 & 51513.5 & 51505.4 & 8.1 & 51489.7 & 51486.2 & 3.5 & -11.9 \\
\hline 6 & 361 & 151209 & 636 & 530 & 80 & 51577.6 & 51569.5 & 8.1 & 51545.2 & 1541.6 & 3.6 & -16.2 \\
\hline 6 & 361 & 151218 & 638 & 530 & 85 & 51665.0 & 51656.9 & 8.1 & 51621.7 & 51618.1 & 3.6 & -21.7 \\
\hline 6 & 361 & 151227 & 640 & 530 & 90 &, 51780.2 & 51772.1 & 8.1 & 51746.3 & 51742.7 & 3.6 & -17.0 \\
\hline 6 & 361 & 151236 & 642 & 530 & 95 & 51971.5 & 51963.4 & 8.1 & 51941.0 & $|51937.4|$ & 3.6 & -15.3 \\
\hline 6 & 361 & 151247 & 644 & 530 & 100 & 52248.8 & 52240.7 & 8.1 & 52260.6 & 52257.0 & 3.6 . & 5.9 \\
\hline 6 & 361 & 151257 & 646 & 530 & 105 & 52610.6 & 52602.4 & 8.2 & 52770.9 & 52767.3 & 3.6 & 80.2 \\
\hline 6 & 361 & 151307 & 648 & 530 & 110 & 52957.7 & 52949.5 & 8.2 & 53309.7 & 53306.1 & 3.6 & 176.0 \\
\hline 6 & 361 & 151318 & 650 & 530. & 115 & 53078.6 & 53070.4 & 8.2 & 53383.0 & 3379.4 & 3.6 & 152.2 \\
\hline 6 & 361 & 151328 & 652 & 530 & 120 & 53042.8 & 53034.6 & 8.2 & 53545.9 & 3542.3 & 3.6 & 251.6 \\
\hline 6 & 361 & 151339 & 654 & 530 & 125 & 52698.3 & 52690.1 & 8.2 & 52964.5 & 52960.9 & 3.6 & 133.1 \\
\hline 6 & 361 & 151349 & 656 & 530 & 130 & 52173.0 & 52164.8 & 8.2 & 51942.4 & 51938.8 & 3.6 & -115.3 \\
\hline 6 & 361 & 151400 & 658 & 530 & 135 & 52123.5 & 52115.2 & 8.3 & 51934.1 & 1930.5 & 3.6 & -94.7 \\
\hline 6 & 361 & 151411 & 660 & 530 & 140 & 52632.1 & 52623.8 & 8.3 & 52934.3 & 52930.6 & 3.7 & 151.1 \\
\hline 6 & 361 & 151422 & 662 & 530 & 145 & 52751.4 & 52743.1 & 8.3 & 54079.9 & 54076.2 & 3.7 & 664.3 \\
\hline 6 & 361 & 151433 & 664 & 530 & 150 & 52286.4 & 52278.1 & 8.3 & 52337.1 & $\mid 52333.4$ & 3.7 & 25.4 \\
\hline 6 & 361 & 151445 & 666 & 530 & 155 & 51762.4 & 51754.1 & 8.3 & .51698 .3 & 1694.6 & 3.7 & -32.1 \\
\hline 6 & 361 & 151456 & 668 & 530 & 160 & 51643.0 & 51634.6 & 8.4 & 51719.2 & 1715.5 & 3.7 & 38.1 \\
\hline 6 & 361 & 151506 & 670 & 530 & 165 & 51514.2 & 51505.8 & 8.4 & 51446.6 & |442.9 & 3.7 & -33.8 \\
\hline 6 & 361 & 151516 & 672 & 530 & 170 & 51460.0 & 51451.6 & 8.4 & 51332.6 & $|51328.9|$ & 3.7 & -63.7 \\
\hline 6 & 361 & 151526 & 674 & 530 & 175 & 51550.4 & 51542.0 & 8.4 & 51329.5 & $|325.8|$ & 3.7 & -110.5 \\
\hline 6 & 361 & 151537 & 676 & 530 & 180 & 51866.4 & 51858.0 & 8.4 & 51714.2 & 710.5 & 3.7 & -76.1 \\
\hline 6 & 361 & 151547 & 678 & 530 & 185 & 52531.8 & 52523.4 & 8.4 & 52509.9 & |52506.2 & 3.7 & -11.0 \\
\hline 6 & 361 & 151558 & 680 & 530 & 190 & 53599.5 & 53591.0 & 8.5 & 54310.9 & 54307.1 & 3.8 & 355.7 \\
\hline 6 & 361 & 151609 & 682 & 530 & 195 & 55107.0 & 55098.5 & 8.5 & & & & * \\
\hline 6 & 361 & 151620 & 684 & 530 & 200 & 54515.3 & 54506.8 & 8.5 & $\bullet$ & $\bullet$ & * & $\bullet$ \\
\hline 6 & 361 & 151631 & 686 & 530 & 205 & 52462.4 & 52453.9 & 8.5 & 52737.8 & 52734.0 & 3.8 & 137.7 \\
\hline 6 & 361 & 151642 & 688 & 530 & 210 & 51076.2 & 51067.7 & 8.5 & 50587.1 & 50583.3 & 3.8 & -244.6 \\
\hline 6 & 361 & 151652 & 690 & 530 & 215 & 50742.2 & 50733.6 & 8.6 & 50415.8 & 50412.0 & 3.8 & -163.2 \\
\hline 6 & 361 & 151703 & 692 & 530 & 220 & 50790.8 & 50782.2 & 8.6 & 50627.3 & 50623.5 & 3.8 & -81.8 \\
\hline 6 & 361 & 151713 & 694 & 530 & 225 & 50943.3 & 50934.7 & 8.6 & 50851.9 & 50848.1 & 3.8 & -45.7 \\
\hline 6 & 361 & 151723 & 696 & 530 & 230 & 56.1 & 51047.5 & 8.6 & 51017.0 & 51013.2 & 3.8 & -19.6 \\
\hline 7 & 361 & 152057 & 698 & 520 & 230 & 50964.4 & 55.4 & 9.0 & 50961.6 & 50957.6 & 4.0 & -1.4 \\
\hline 7 & 361 & 152108 & 700 & 520 & 225 & 50800.6 & 50791.6 & 9.0 & 50782.1 & 50778.1 & 4.0 & -9.3 \\
\hline 7 & 361 & 152118 & 702 & 520 & 220 & 50508.2 & 50499.2 & 9.0 & 50416.4 & 50412.4 & $4.0^{\circ}$ & -45.9 \\
\hline 7 & 361 & 152129 & 704 & 520 & 215 & 50087.8 & 50078.8 & 9.0 & 49861.3 & 49857.3 & 4.0 & -113.3 \\
\hline 7 & 361 & 152140 & 706 & 520 & 210 & 49571.3 & 49562.3 & 9.0 & 49139.0 & 49134.9 & 4.1 & -216.2 \\
\hline 7 & 361 & 152150 & 708 & 520 & 205 & 48950.7 & 48941.6 & 9.1 & 48076.2 & 48072.1 & 4.1 & -437.3 \\
\hline 7 & 361 & 152200 & 710 & 520 & 200 & 48945.4 & 48936.3 & 9.1 & - & * & - & * \\
\hline 7 & 361 & 152210 & 712 & 520 & 195 & 49983.3 & 49974.2 & 9.1 & 49117.9 & 49113.8 & 4.1 & -432.7 \\
\hline 7 & 361 & 152220 & 714 & 520 & 190 & 50619.3 & 50610.2 & 9.1 & 50420.6 & 50416.5 & 4.1 & -99.4 \\
\hline 7 & 361 & 152230 & 716 & 520 & 185 & 50919.6 & 50910.5 & 9.1 & 50754.5 & 50750.4 & 4.1 & -82.6 \\
\hline 7 & 361 & 152240 & 718 & 520 & 180 & 51010.8 & 51001.6 & 9.2 & 50849.5 & 50845.4 & 4.1 & -80.7 \\
\hline 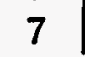 & 361 & 152250 & 720 & 52 & 175 & 51057.7 & 51048.5 & 9.2 & 50909.6 & 50905.5 & 4.1 & -74.1 \\
\hline
\end{tabular}




\begin{tabular}{|c|c|c|c|c|c|c|c|c|c|c|c|c|}
\hline Line & | Day & Time & Sta. & $\mathbf{x}$ & $\mathbf{Y}$ & $\begin{array}{r}T \\
\text { uncorr. }\end{array}$ & $\begin{array}{l}\mathrm{P}(\mathrm{nT}) \\
\text { corr. }\end{array}$ & diff. & $\begin{array}{r}\text { BOT } \\
\text { uncorr. }\end{array}$ & $\begin{array}{c}\text { TOM (nT) } \\
\text { corr. }\end{array}$ & diff. & $\begin{array}{c}\text { Vert.Grad. } \\
\text { (nT/ft) }\end{array}$ \\
\hline 7 & 361 & 152301 & $\overline{722}$ & 520 & 170 & 51179.1 & 51169.9 & $\overline{9.2}$ & 51060.3 & 51056.2 & 4.1 & -59.4 \\
\hline 7 & 361 & 152310 & 724 & 520 & 165 & 51255.0 & 51245.8 & 9.2 & 51269.7 & 51265.6 & 4.1 & 7.4 \\
\hline 7 & 361 & 152321 & 726 & 520 & 160 & 51084.1 & 51074.9 & 9.2 & 50760.9 & 50756.8 & 4.1 & -161.6 \\
\hline 7 & 361 & 152331 & 728 & 520 & 155 & 51065.0 & 51055.8 & 9.2 & 50242.4 & 50238.2 & 4.2 & -411.3 \\
\hline 7 & 361 & 152342 & 730 & 520 & 150 & 51675.8 & 51666.5 & 9.3 & 51241.3 & 51237.1 & 4.2 & -217.3 \\
\hline 7 & 361 & 152352 & 732 & 520 & 145 & 52616.6 & 52607.3 & 9.3 & 53300.8 & 53296.6 & 4.2 & 342.1 \\
\hline 7 & 361 & 152403 & 734 & 520 & 140 & 52618.3 & 52609.0 & 9.3 & 52962.2 & 52958.0 & 4.2 & 172.0 \\
\hline 7 & 361 & 152413 & 736 & 520 & 135 & 52090.9 & 520081.6 & 9.3 & 51807.7 & 51803.5 & 4.2 & -141.6 \\
\hline 7 & 361 & 152423 & 738 & 520 & 130 & 52136.1 & 52126.8 & 9.3 & 51773.0 & 51768.8 & 4.2 & -181.6 \\
\hline 7 & 361 & 152433 & 740 & 520 & 125 & 52804.0 & 52794.7 & 9.3 & 53159.6 & 53155.4 & 4.2 & 177.8 \\
\hline 7 & 361 & 152443 & 742 & 520 & 120 & 52851.6 & 52842.2 & 9.4 & 52741.8 & 527.37 .6 & 4.2 & -54.9 \\
\hline 7 & 361 & 152454 & 744 & 520 & 115 & 53077.9 & 53068.5 & 9.4 & 53165.2 & 53161.0 & 4.2 & 43.7 \\
\hline 7 & 361 & 152504 & 746 & 520 & 110 & 53615.2 & 53605.8 & 9.4 & 54433.0 & 54428.8 & 4.2 & 408.9 \\
\hline 7 & 361 & 152514 & 748 & 520 & 105 & 53442.1 & 53432.7 & 9.4 & 54180.0 & 54175.8 & 4.2 & 369.0 \\
\hline 7 & 361 & 152525 & 750 & 520 & 100 & 52789.7 & 52780.3 & 9.4 & 52952.5 & 52948.2 & 4.3 & 81.4 \\
\hline 7 & 361 & 152535 & 752 & 520 & 95 & 52208.6 & 52199.2 & 9.4 & 52214.4 & 52210.1 & 4.3 & 2.9 \\
\hline 7 & 361 & 152544 & 754 & 520 & 90 & 51863.8 & 51854.3 & 9.5 & 51843.3 & 51839.0 & 4.3 & -10.3 \\
\hline 7 & 361 & 152554 & 756 & 520 & 85 & 51685.2 & $|51675.7|$ & 9.5 & 51650.5 & $\mid 51646.2$ & 4.3 & -17.4 \\
\hline 7 & 361 & 152604 & 758 & 520 & 80 & 51573.5 & 51564.0 & 9.5 & 51539.4 & 51535.1 & 4.3 & -17.1 \\
\hline 7 & 361 & 152614 & 760 & 520 & 75 & 51491.1 & 51481.6 & 9.5 & 51473.6 & 51469.3 & 4.3 & -8.8 \\
\hline 7 & 361 & 152624 & 762 & 520 & 70 & 51437.9 & 51428.4 & 9.5 & 51421.5 & 51417.2 & 4.3 & -8.2 \\
\hline 7 & 361 & 152634 & 764 & 520 & 65 & 51387.5 & 51378.0 & 9.5 & 51369.5 & 51365.2 & 4.3 & -9.0 \\
\hline 7 & 361 & 152644 & 766 & 520 & 60 & 51337.5 & 51327.9 & 9.6 & 51306.4 & 51302.1 & 4.3 & -15.6 \\
\hline 7 & 361 & 152654 & 768 & 520 & 55 & 51268.4 & 51258.8 & 9.6 & 51220.5 & 51216.2 & 4.3 & -24.0 \\
\hline 7 & 361 & 152704 & 770 & 520 & 50 & 51213.0 & 51203.4 & 9.6 & 51073.1 & 51068.8 & 4.3 & -70.0 \\
\hline 7 & 361 & 152714 & 77.2 & 520 & 45 & 51378.0 & 51368.4 & 9.6 & 51157.4 & 51153.0 & 4.4 & -110.3 \\
\hline 7 & 361 & 152725 & 774 & 520 & 40 & 52046.7 & 52037.1 & 9.6 & 52808.9 & 52804.5 & 4.4 & 381.1 \\
\hline 7 & 361 & 152735 & 776 & 520 & 35 & 52297.3 & 52287.6 & 9.7 & 52547.5 & 52543.1 & 4.4 & 125.1 \\
\hline 7 & 361 & 152746 & 778 & 520 & 30 & 52438.2 & 52428.5 & 9.7 & 52241.4 & 52237.0 & 4.4 & -98.4 \\
\hline 7 & 361 & 152757 & 780 & 520 & 25 & 52911.3 & 52901.6 & 9.7 & 53512.4 & $53508.0 \mid$ & 4.4 & 300.6 \\
\hline 7 & 361 & 152807 & 782 & 520 & 20 & 52522.3 & 52512.6 & 9.7 & 52632.6 & 52628.2 & 4.4 & 55.2 \\
\hline 7 & 361 & 152818 & 784 & 520 & 15 & 52014.3 & 52004.6 & 9.7 & 51909.4 & 51905.0 & 4.4 & -52.5 \\
\hline 7 & 361 & 152830 & 786 & 520 & 10 & 51753.3 & 51743.6 & 9.7 & 51683.0 & 51678.6 & 4.4 & -35.2 \\
\hline 7 & 361 & 152842 & 788 & 520 & 5 & 51623.4 & 51613.6 & 9.8 & 51597.0 & 51592.6 & 4.4 & -13.2 \\
\hline 7 & 361 & 152853 & 790 & 520 & 0 & 51558.2 & 51548.4 & 9.8 & 51548.5 & 51544.1 & 4.4 & -4.9 \\
\hline 8 & 361 & 153420 & 802 & 510 & 0 & 51527.7 & 51521.2 & 6.5 & 51494.1 & 51492.9 & 1.2 & -16.8 \\
\hline 8 & 361 & 153434 & 804 & 510 & 5 & 51599.3 & $|51592.9|$ & 6.4 & 51533.6 & 51532.6 & 1.0 & -32.9 \\
\hline 8 & 361 & 153445 & 806 & 510 & 10 & 51719.2 & $51713.0 \mid$ & 6.2 & 51627.6 & 51626.7 & 0.9 & -45.8 \\
\hline 8 & 361 & 153456 & 808 & 510 & 15 & 52023.2 & 52017.1 & 6.1 & 51896.0 & 51895.2 & 0.8 & -63.6 \\
\hline 8 & 361 & 153507 & 810 & 510 & 20 & 52830.9 & 52825.0 & 5.9 . & $52917: 1$ & 52916.5 & 0.6 & 43.1 \\
\hline 8 & 361 & 153518 & 812 & 510 & 25 & 53694.7 & 53688.9 & 5.8 & 54843.8 & 54843.3 & 0.5 & 574.6 \\
\hline 8 & 361 & 153530 & 814 & 510 & 30 & 53316.3 & 53310.7 & 5.6 & 54479.8 & 54479.5 & 0.3 & 581.8 \\
\hline 8 & 361 & 153540 & 816 & 510 & 35 & 52427.1 & 52421.6 & 5.5 & 52587.6 & 52587.4 & 0.2 & 80.3 \\
\hline 8 & 361 & 153550 & 818 & 510 & 40 & 51647.7 & $51642.3 \mid$ & 5.4 & 51333.1 & 51333.0 & 0.1 & -157.3 \\
\hline 8 & 361 & 153601 & 820. & 510 & 45 & 51233.0 & 51227.8 & 5.2 & 50890.9 & 50890.9 & 0.0 & -171.1 \\
\hline 8 & 361 & 153611 & 822 & 510 & 50 & 51170.1 & 51165.0 & 5.1 & 51004.5 & 51004.7 & -0.2 & -82.8 \\
\hline 8 & 361 & 153620 & 824 & 510 & 55 & 51227.8 & 51222.8 & 5.0 & 51150.8 & 51151.1 & -0.3 & -38.5 \\
\hline 8 & 361 & 153630 & 826 & $\mid 510$ & 60 & 51291.2 & 51286.3 & 4.9 & 51245.4 & 51245.8 & -0.4 & -22.9 \\
\hline 8 & 361 & 153639 & 828 & 510 & 65 & 51344.8 & 51340.0 & 4.8 & 51307.8 & 51308.3 & -0.5 & -18.5 \\
\hline
\end{tabular}




\begin{tabular}{|c|c|c|c|c|c|c|c|c|c|c|c|c|}
\hline & & & & & & & & & & OI & & ned \\
\hline Line & Day & Time & Sta. & $\mathbf{x}$ & $\mathbf{Y}$ & ncorr. & corr. & diff. & incorr. & corr. & diff. & \\
\hline 8 & 361 & 153648 & 830 & 510 & 70 & 51391.2 & 51386.6 & 4.6 & 51354.1 & 51354.8 & -0.7 & -18.6 \\
\hline 8 & 361 & 153658 & 832 & 510 & 75 & 51445.2 & 51440.7 & 4.5 & 51400.1 & 51400.9 & -0.8 & -22.6 \\
\hline 8 & 361 & 153708 & 834 & 510 & 80 & 51515.7 & 51511.3 & 4.4 & 51455.5 & 51456.4 & -0.9 & -30.1 \\
\hline 8 & 361 & 153718 & 836 & 510 & 85 & 51623.6 & 51619.3 & 4.3 & 51539.7 & 51540.7 & -1.0 & -42.0 \\
\hline 8 & 361 & 153728 & 838 & 510 & 90 & 51819.5 & 51815.4 & 4.1 & 51713.5 & 51714.6 & -1.1 & -53.0 \\
\hline 8 & 361 & 153739 & 840 & 510 & 95 & 52166.9 & 52162.9 & 4.0 & 52045.9 & 52047.2 & -1.3 & -60.5 \\
\hline 8 & 361 & 153749 & 842 & 510 & 100 & 52753.5 & 52749.6 & 3.9 & 52703.5 & 52704.9 & -1.4 & -25.0 \\
\hline 8 & 361 & 153800 & 844 & 510 & 105 & 53397.9 & 53394.2 & 3.7 & 53597.9 & 53599.5 & -1.6 & 100.0 \\
\hline 8 & 361 & $153811^{\circ}$ & 846 & 510 & 110 & 53365.8 & 53362.2 & 3.6 & 53567.0 & 53568.7 & -1.7 & 100.6 \\
\hline 8 & 361 & 153823 & 848 & 510 & 115 & 52752.6 & 52749.2 & 3.4 & 52780.6 & 52782.4 & -1.8 & 14.0 \\
\hline 8 & 361 & 153833 & 850 & 510 & 120 & .52309 .3 & 52306.0 & 3.3 & 52839.6 & 52841.6 & -2.0 & 265.2 . \\
\hline 8 & 361 & 153843 & 852 & 510 & 125 & $51814.5^{\prime}$ & 51811.3 & 3.2 & 52052.0 & 52054.1 & -2.1 & 118.8 \\
\hline 8 & 361 & 153854 & 854 & 510 & 130 & 51507.2 & 51504.2 & 3.0 & 51140.6 & 51142.8 & -2.2 & -183.3 \\
\hline 8 & 361 & 153905 & 856 & 510 & 135 & 51858.0 & 51855.1 & $2.9^{\circ}$ & 51655.1 & 51657.5 & -2.4 & -101.5 \\
\hline 8 & 361 & 153916 & 858 & 510 & 140 & 52459.6 & 52456.9 & 2.7 & 52770.4 & 52772.9 & -2.5 & 155.4 \\
\hline 8 & 361 & 153926 & 860 & 510 & 145 & 52401.9 & 52399.3 & 2.6 & 52742.1 & 52744.8 & -2.7 & 170.1 \\
\hline 8 & 361 & 153938 & 862 & 510 & 150 & 51819.1 & 51816.7 & 2.4 & 51902.7 & 51905.5 & -2.8 & 41.8 \\
\hline 8 & 361 & 153950 & 864 & 510 & 155 & 09.0 & 51406.7 & 2.3 & 51258.9 & 51261.8 & -2.9 & -75.1 \\
\hline 8 & 361 & 154002 & 866 & 510 & 160 & 51326.2 & 51324.1 & 2.1 & 51341.8 & 51344.9 & -3.1 & 7.8 \\
\hline 8 & 361 & 154013 & 868 & 510 & 165 & 51235.2 & 51233.2 & 2.0 & 51247.4 & 51250.6 & -3.2 & 6.1 \\
\hline 8 & 361 & 154024 & 870 & 510 & 170 & 50942.9 & 50941.1 & 1.8 & 50575.4 & 50578.8 & -3.4 & -183.8 \\
\hline 8 & 361 & 154035 & 872 & 510 & 175 & 50741.6 & 50739.9 & 1.7 & 50585.9 & 50589.4 & $-3,5$ & -77.9 \\
\hline 8 & 361 & 154045 & 874 & 510 & 180 & 50532.0 & 50530.4 & 1.6 & 50407.4 & 50411.0 & -3.6 & -62.3 \\
\hline 8 & 361 & 154056 & 876 & 510 & 185 & 50242.8 & 50241.4 & 1.4 & 50179.1 & 50182.9 & -3.8 & -31.9 \\
\hline 8 & 361 & 154107 & 878 & 510 & 190 & 49442.7 & 49441.4 & 1.3 & 49323.9 & 49327.8 & -3.9 & -59.4 \\
\hline 8 & 361 & 154118 & 880 & 51 & 195 & 30.0 & 48028.9 & 1.1 & 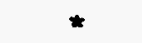 & & ๘. & * \\
\hline 8 & 361 & 154130 & 882 & 51 & 200 & 23.8 & 47322.8 & 1.0 & * & & * & * \\
\hline 8 & 361 & 154141 & 884 & 510 & 205 & 47994.4 & 47993.5 & 0.9 & 47427.1 & 47431.5 & -4.4 & -283.7 \\
\hline 8 & 361 & 154152 & 886 & 510 & 210 & 49228.1 & 49227.4 & 0.7 & 48940.7 & 48945.2 & -4.5 & -143.7 \\
\hline 8 & 361 & 154202 & 888 & 510 & 215 & 49981.6 & 49981.0 & 0.6 & 49869.3 & 49873.9 & -4.6 & -56.2 \\
\hline 8 & 361 & 154213 & 890 & 51 & 220 & & 488.7 & 0.4 & 50472.5 & 477.3 & -4.8 & -8.3 \\
\hline 8 & 361 & 154224 & .892 & 510 & 225 & 02.5 & 50802.2 & 0.3 & 50817.4 & 50822.3 & -4.9 & 7.5 \\
\hline 8 & 361 & 154234 & 894 & 510 & 230 & 50975.0 & 50974.8 & 0.2 & 50987.0 & 50992.0 & -5.0 & 6.0 \\
\hline 9 & 361 & 154529 & 896 & 500 & 230 & 51079.5 & 51081.6 & -2.1 & 51111.1 & 51118.4 & -7.3 & 15.8 \\
\hline 9 & 361 & 154539 & 898 & 500 & .225 & 50935.7 & 50937.9 & -2.2 & 50980.8 & 50988.2 & -7.4 & 22.6 \\
\hline 9 & 361 & 154548 & 900 & 500 & 220 & 50686.8 & 50689.1 & -2.3 & 50684.5 & 50692.0 & -7.5 & -1.2 \\
\hline 9 & 361 & 154558 & 902 & 500 & 215 & 50223.1 & 50225.6 & -2.5 & 50124.0 & 50131.6 & -7.6 & -49.6 \\
\hline 9 & 361 & 154609 & 904 & 500 & 210 & 49589.2 & 49591.8 & -2.6 & 49248.4 & 49256.1 & -7.7 & -170.4 \\
\hline 9 & 361 & 154620 & 906 & 50 & 205 & 49006.1 & 49008.9 & -2.8 & 48739.9 & 48747.8 & -7.9 & -133.1 \\
\hline 9 & 361 & 154630 & 908 & 50 & 200 & 31.6 & 48584.5 & -2.9 & 47809.0 & 47817.0 & -8.0 & -386.3 \\
\hline 9 & 361 & 154640 & 910 & 50 & 195 & 48897.6 & 48900.6 & -3.0 & 48214.7 & 48222.9 & -8.2 & -341.5 \\
\hline 9 & 361 & 154651 & 912 & 500 & 190 & 49763.1 & 49766.3 & -3.2 & 49475.8 & 49484.1 & -8.3 & -143.7 \\
\hline 9 & 361 & 154701 & 914 & 500 & 185 & 50288.4 & 50291.7 & -3.3 & 49917.2 & 49925.6 & -8.4 & -185.6 \\
\hline 9 & 361 & 154711 & 916 & 500 & 180 & 50577.2 & 50580.6 & $-3.4 \mid$ & 50442.7 & 50451.2 & -8.5 & -67.3 \\
\hline 9 & 361 & 154721 & 918 & 500 & 175 & 50749.8 & 50753.3 & -3.5 & 50592.2 & 50600.9 & -8.7 & -78.8 \\
\hline 9 & 361 & 154731 & 920 & 500 & 170 & 50969.2 & 50972.9 & -3.7 & 50584.9 & 50593.7 & -8.8 & -192.2 \\
\hline 9 & 361 & 154742 & 922 & 500 & 165 & 51570.6 & 51574.4 & -3.8 & 52137.0 & 52145.9 & -8.9 & 283.2 \\
\hline 9 & 361 & 154752 & 924 & 500 & 160 & 51518.7 & 51522.6 & -3.9 & 51652.8 & 51661.9 & -9.1 & 67.1 \\
\hline 0 & 361 & 154802 & 926 & 500 & 155 & 51336.6 & 51340.7 & -4.1 & 50923.6 & 50932.8 & -9.2 & -206.5 \\
\hline
\end{tabular}




\begin{tabular}{|c|c|c|c|c|c|c|c|c|c|c|c|c|}
\hline & & & & & & & (nT) & & BOT & TOM (n) & & Vert.Grad. \\
\hline Line & Day & Time & Sta. & $\mathbf{X}$ & $\mathbf{Y}$ & uncorr. & corr. & diff. & uncorr. & corr. & diff. & (nT/ft) \\
\hline 9 & 361 & 154811 & 928 & 500 & 150 & 51641.6 & 51645.8 & -4.2 & 51317.0 & 51326.3 & -9.3 & -162.3 \\
\hline 9 & 361 & 154822 & 930 & 500 & 145 & 285.7 & 52290.0 & -4.3 & 2570.5 & 52579.9 & -9.4 & 142.4 \\
\hline 9 & 361 & 154833 & 932 & 500 & 140 & 52386.4 & 52390.9 & -4.5 & 53006.5 & 53016.1 & -9.6 & 310.1 \\
\hline 9 & 361 & 154844 & 934 & 500 & $135^{\circ}$ & 51519.6 & 51524.2 & -4.6 & 51266.7 & 51276.4 & -9.7 & -126.5 \\
\hline 9 & 361 & 154855 & 936 & 500 & 130 & 50594.0 & 50598.8 & -4.8 & 50120.8 & 50130.7 & -9.9 & -236.6 \\
\hline 9 & 361 & 154905 & 938 & 500 & 125 & 50258.1 & 50263.0 & -4.9 & 49039.9 & 49049.9. & -10.0 & -609.1 \\
\hline 9 & 361 & 154916 & 940 & 500 & 120 & 50312.2 & 50317.2 & -5.0 & 48953.9 & 48964.0 & $\mid-10.1$ & -679.2 \\
\hline 9 & 361 & 154927 & 942 & 500 & 115 & 52135.9 & 52141.1 & -5.2 & 58607.4 & 58617.7 & $|-10.3|$ & 3235.8 \\
\hline 9 & 361 & 154937 & 944 & 500 & 110 & 55951.7 & 55957.0 & -5.3 & 58557.6 & 58568.0 & $\mid-10.4$ & 1303.0 \\
\hline 9 & 361 & 154948 & 946 & 500 & 105 & 55812.8 & 55818.2 & -5.4 & 58548.7 & 58559.2 & $\mid-10.5$ & 1368.0 \\
\hline 9 & 361 & 154959 & 948 & 500 & 100 & 53248.5 & 53254.1 & -5.6 & 52979.3 & 52990.0 & -10.7 & -134.6 \\
\hline 9 & 361 & 155010 & 950 & 500 & 95 & 52058.3 & 52064.0 & -5.7 & 51765.6 & 51776.4 & $\mid-10.8$ & -146.4 \\
\hline 9 & 361 & 155021 & 952 & 500 & 90 & 51640.0 & 51645.9 & -5.9 & 51464.9 & 51475.8 & -10.9 & -87.6 \\
\hline 9 & 361 & 155031 & 954 & 500 & 85 & 51470.3 & 51476.3 & -6.0 & 51373.9 & 51385.0 & -11.1 & -48.2 \\
\hline 9 & 361 & 047 & 956 & 500 & 80 & 4.2 & 51400.4 & -6.2 & 51339.9 & 351.2 & $\mid-11.3$ & -27.2 \\
\hline 9 & 361 & 058 & 958 & 500 & 75 & 4.7 & 51351.0 & -6.3 & 51309.7 & 321.1 & -11.4 & -17.5 \\
\hline 9 & 361 & 155108 & 960 & 500 & 70 & 51305.1 & 51311.6 & -6.5 & 51283.5 & 1295.0 & -11.5 & -10.8 \\
\hline 9 & 361 & 155133 & 962 & 500 & 65 & 51261.4 & 51268.2 & -6.8 & 51241.9 & 51253.8 & -11.9 & -9.8 \\
\hline 9 & 361 & 155143 & 964 & 500 & 60 & 51204.0 & 51210.9 & -6.9 & 51174.4 & 51186.4 & -12.0 & -14.8 \\
\hline 9 & 361 & 155154 & 966 & 500 & 55 & 51134.6 & 51141.7 & -7.1 & 51059.7 & 51071.8 & -12.1 & -37.5 \\
\hline 9 & 361 & 155204 & 968 & 500 & 50 & 51096.3 & 51103.5 & -7.2 & 50911.7 & 50924.0 & -12.3 & -92.3 \\
\hline 9 & 361 & 155216 & 970 & 500 & 45 & 51262.8 & 51270.2 & -7.4 & 50786.6 & 0799.0 & -12.4 & -238.1 \\
\hline 9 & 361 & 155227 & 972 & 500 & 40 & 51898.5 & 51906.0 & -7.5 & 51452.8 & 1465.3 & -12.5 & -222.9 \\
\hline 9 & 361 & 155238 & 974 & 500 & 35 & 52476.5 & 52484.1 & -7.6 & 52272.1 & 52284.8 & -12.7 & -102.2 \\
\hline 9 & 361 & 155250 & 976 & 500 & 30 & 53031.3 & 53039.1 & -7.8 & 53487.4 & 53500.2 & -12.8 & 228.1 \\
\hline 9 & 361 & 155301 & 978 & 500 & 25 & 53028.8 & 53036.7 & -7.9 & 54050.3 & 54063.3 & -13.0 & 510.8 \\
\hline 9 & 361 & 155311 & 980 & 500 & 20 & 52355.2 & 52363.3 & -8.1 & 52280.0 & $52293: 1$ & -13.1 & -37.6 \\
\hline 9 & 361 & 155324 & 982 & 500 & 15. & 51804.3 & 51812.5 & -8.2 & 51655.8 & 51669.1 & -13.3 & -74.3 \\
\hline 9 & 361 & 155340 & 984 & 500 & 10 & 51577.0 & 51585.4 & -8.4 & 51493.3 & 51506.8 & -13.5 & -41.9 \\
\hline 9 & 361 & 155350 & 986 & 50 & $5^{\circ}$ & 51500.9 & 51509.5 & -8.6 & 51452.6 & 4466.2 & -13.6 & -24.2 \\
\hline 9 & 361 & 155401 & 988 & 500 & 0 & 51469.0 & 51477.7 & -8.7 & 51443.8 & 51457.5 & -13.7 & -12.6 \\
\hline 10 & 362 & 357 & 1014 & 490 & 0 & 51428.8 & 51434.8 & -6.0 & 51405.0 & 51419.5 & -14.5 & -11.9 \\
\hline 10 & 362 & 105406 & 1016 & 490 & 5 & 51454.0 & 51460.1 & -6.1 & 51409.8 & 51424.3 & -14.5 & -22.1 \\
\hline 10 & 362 & 105415 & 1018 & 490 & 10 & 51470.4 & 51476.5 & -6.1 & 51393.4 & 51407.9 & -14.5 & -38.5 \\
\hline 10 & 362 & 105423 & 1020 & 490 & 15 & 51567.2 & 51573.4 & -6.2 & $5,1434.6$ & 51449.1 & -14.5 & -66.3 \\
\hline 10 & 362 & 105432 & 1022 & 490 & 20 & 51775.8 & 51782.0 & -6.2 & .51615 .4 & 51629.9 & -14.5 & -80.2 \\
\hline 10 & 362 & 105442 & 1024 & 490 & 25 & 52009.2 & 52015.5 & -6.3 & 51696.8 & 51711.2 & -14.4 & -156.2 \\
\hline 10 & 362 & 105451 & 1026 & 490 & 30 & 52256.8 & 52263.1 & -6.3 & 51700.2 & 51714.6 & -14.4 & -278.3 \\
\hline 10 & 362 & 105502 & 1028 & 490 & 35 & 53026.8 & 53033.2 & -6.4 & 54003.6 & 54018.0 & -14.4 & 488.4 \\
\hline 10 & 362 & 105511 & 1030 & 490 & 40 & 52566.8 & 52573.2 & -6.4 & 53104.0 & 53118.4 & -14.4 & 268.6 \\
\hline 10 & 362 & 105521 & 1032 & 490 & 45 & 51289.0 & 51295.5 & -6.5 & 50738.6 & 50753.0 & -14.4 & -275.2 \\
\hline 10 & 362 & 105548 & 1034 & 490 & 50 & 50970.4 & 50977.1 & -6.7 & 50714.2 & 50728.6 & -14.4 & -128.1 \\
\hline 10 & 362 & 105557 & 1036 & 490 & 55 & 51038.6 & 51045.3 & -6.7 & 50936.6 & 50951.0 & -14.4 & -51.0 \\
\hline 10 & 362 & 105606 & 1038 & 490 & 60 & 51130.4 & 51137.2 & -6.8 & 51082.4 & 51096.8 & -14.4 & -24.0 \\
\hline 10 & 362 & 105615 & 1040 & 490 & 65 & 51208.4 & 51215.2 & -6.8 & 51173.2 & 51187.6 & -14.4 & -17.6 \\
\hline 10 & 362 & 105623 & 1042 & 490 & 70 & 51248.0 & 51254.9 & -6.9 & 51214.8 & 51229.2 & -14.4 & -16.6 \\
\hline 10 & 362 & 105631 & 1044 & 490 & 75 & 51276.2 & 51283.1 & -6.9 & 51229.2 & 51243.6 & -14.4 & -23.5 \\
\hline 10 & 362 & 105639 & 1046 & 490 & 80 & 51291.8 & 51298.8 & -7.0 & 51219.0 & 51233.4 & -14.4 & -36.4 \\
\hline 10 & 362 & 105647 & 1048 & 490 & 85 & 51323.6 & 51330.6 & -7.0 & 51195.8 & 51210.2 & -14.4 & -63.9 \\
\hline
\end{tabular}




\begin{tabular}{|c|c|c|c|c|c|c|c|c|c|c|c|c|}
\hline & & & & & & & (T) & & & $\mathrm{OM}$ & & $\mathrm{irad}$ \\
\hline ne & ay & Time & Sta. & $\mathbf{x}$ & $\mathbf{Y}$ & corr. & corr. & diff. & corr. & corr. & diff. & \\
\hline 10 & 362 & 105656 & 1050 & 490 & 90 & 51431.4 & 51438.5 & -7.1 & 51196.0 & 51210.4 & -14.4 & -117.7 \\
\hline 10 & 362 & 105705 & 1052 & 490 & 95 & 51847.8 & 51854.9 & -7.1 & 51403.2 & |51417.6 & $\mid-14.4$ & -222.3 \\
\hline 10 & 362 & 105714 & 1054 & 490 & 100 & 53149.2 & 53156.4 & -7.2 & 52564.6 & 52579.0 & $\mid-14.4$ & -292.3 \\
\hline 10 & 362 & 105723 & 1056 & 490 & 105 & 52208.8 & 52216.0 & -7.2 & 17327.0 & 17341.4 & $\mid-14.4$ & -17440.9 \\
\hline 10 & 362 & 105732 & 1058 & 490 & 110 & 30916.0 & 30923.3 & -7.3 & 8111.0 & 8125.4 & $\mid-14.4$ & -11402.5 \\
\hline 10 & 362 & 105741 & 1060 & 490 & 115 & 51359.4 & 51366.7 & -7.3 & 32350.8 & 32365.2 & -14.4 & -9504.3 \\
\hline 10 & 362 & 105749 & 1062 & 490 & 120 & 49521.6 & 49529.0 & -7.4 & 18389.2 & 18403.5 & $\mid-14.3$ & -15566.2 \\
\hline 10 & 362 & 105757 & 1064 & 490 & 125 & 50002.8 & 50010.2 & -7.4 & 49127.4 & 49141.7 & -14.3 & -437.7 \\
\hline 10 & 362 & 105806 & 1066 & 490 & 130 & 50745.2 & 50752.7 & -7.5 & 50398.4 & 50412.7 & -14.3 & -173.4 \\
\hline 10 & 362 & 105814 & 1068 & 490 & 135 & 51741.0 & 51748.5 & $=7.5$ & 51711.0 & 51725.3 & $\mid-14.3$ & -15.0 \\
\hline 10 & 362 & 105822 & $1070^{\circ}$ & 490 & 140 & 52610.4 & 52618.0 & -7.6 & 53156.4 & 53170.7 & $\mid-14.3$ & 273.0 \\
\hline 10 & 362 & 105831 & 1072 & 490 & 145 & 52488.2 & 52495.8 & -7.6 & 52878.4 & 52892.7 & -14.3 & 195.1 \\
\hline 10 & 362 & 105840 & 1074 & 490 & 150 & 51744.0 & 51751.7 & -7.7 & 51679.8 & 51694.1 & -14.3 & -32.1 \\
\hline 10 & 362 & 105849 & 1076 & 490 & 155 & 9.8 & 51167.5 & -7.7 & 50743.2 & 50757.5 & -14.3 & -208.3 \\
\hline 10 & 362 & 105858 & 1078 & 490 & 160 & 9.6 & 51117.4 & -7.8 & 50825.8 & 50840.1 & $\mid-14.3$ & -141.9 \\
\hline 10 & 362 & 105907 & 1080 & 490 & 165 & 56.6 & 51264.4 & -7.8 & 51627.6 & 1641.9 & -14.3 & 185.5 \\
\hline 10 & 362 & 105915 & 1082 & 490 & 170 & 986.8 & 50994.7 & -7.9 & 50795.6 & 50809.9 & -14.3 & -95.6 \\
\hline 10 & 362 & 105923 & 1084 & 490 & 175 & 884.8 & 50892.7 & -7.9 & 50701.6 & 50715.9 & -14.3 & -91.6 \\
\hline 10 & 362 & 105932 & 1086 & 490 & 180 & 08.4 & 50916.4 & -8.0 & 50752.6 & 50766.9 & $\mid-14.3$ & -77.9 \\
\hline 10 & 362 & 105940 & 1088 & 490 & .185 & 22.4 & 51030.4 & -8.0 & 51045.2 & 51059.5 & $\mid-14.3$ & 11.4 \\
\hline 10 & 362 & 105948 & 1090 & 490 & 190 & 50914.4 & 50922.5 & -8.1 & 51141.4 & $\mid 51155.7$ & $\mid-14.3$ & 113.5 \\
\hline 10 & 362 & 105957 & 1092 & 490 & 195 & 043.0 & 50051.1 & -8.1 & 49488.6 & 49502.9 & $\mid-14.3$ & -277.2 \\
\hline 10 & 362 & 110007 & 1094 & 490 & 200 & 49477.6 & 49485.8 & -8.2 & 27759.8 & 27774.1 & -14.3 & -10858.9 \\
\hline 10 & 362 & 110016 & 1096 & 490 & 205 & 49985.6 & 49993.8 & -8.2 & 49367.4 & 49381.7 & -14.3 & -309.1 \\
\hline 10 & 362 & 110025 & 1098 & 490 & 210 & 50454.6 & 50462.9 & -8.3 & 50093.8 & 50108.1 & $\mid-14.3$ & -180.4 \\
\hline 10 & 362 & 110033 & 1100 & 490 & 215 & 50780.2 & 50788.5 & -8.3 & 50703.6 & 50717.9 & -14.3 & -38.3 \\
\hline 10 & 362 & 110042 & 1102 & 490 & 220 & 2.2 & 51010.6 & -8.4 & 51007.2 & 51021.5 & -14.3 & 2.5 \\
\hline 10 & 362 & 110050 & 1104 & 490 & 225 & 5.8 & 51124.2 & -8.4 & 51137.8 & 51152.1 & -14.3 & 11.0 \\
\hline 10 & 362 & 110058 & 1106 & 490 & 230 & 51182.8 & 51191.3 & -8.5 & $51201.2^{\circ}$ & 51215.4 & $\mid-14.2$ & 9.2 \\
\hline 11 & 362 & 110449 & 1108 & 480 & 230 & 8.6 & 51248.4 & -9.8 & 51248.0 & 62.1 & -14.1 & 4.7 \\
\hline 11 & 362 & 110459 & 1110 & 480 & 225 & 9.4 & 51249.3 & -9.9 & 51224.6 & $\mid 51238.7$ & $|-14.1|$ & -7.4 \\
\hline 11 & 362 & 110507 & 1112 & 480 & 220 & $51323 . \dot{6}$ & 51333.5 & -9.9 & 51261.0 & 51275.1 & -14.1 & -31.3 \\
\hline 11 & 362 & 110516 & 1114 & 480 & 215 & 714.4 & 51724.4 & -10.0 & 51647.4 & 51661.5 & $\mid-14.1$ & $=33.5$ \\
\hline 11 & 362 & 110524 & 1116 & 480 & 210 & 52758.4 & 52768.4 & -10.0 & 53372.8 & 53386.9 & -14.1 & 307.2 \\
\hline 11 & 362 & 110534 & 1118 & 480 & -205 & 53604.4 & 53614.5 & $|-10.1|$ & 55139.0 & 55153.1 & -14.1 & 767.3 \\
\hline 11 & 362 & 110542 & 1120 & 480 & 200 & 5.2 & 52985.3 & -10.1 & 53573.2 & 53587.3 & $\mid-14.1$ & 299.0 \\
\hline 11 & 362 & 110551 & 1122 & 480 & 195 & 3.0 & 52533.2 & -10.2 & 52701.4 & 52715.5 & $\mid-14.1$ & 89.2 \\
\hline 11 & 362 & 110600 & 1124 & 480 & 190 & 9.4 & 52299.6 & -10.2 & 52453.8 & 52467.9 & -14.1 & 82.2 \\
\hline 11. & 362 & 110609 & 1126 & 480 & 185 & 51879.8 & 51890.1 & -10.3 & 51851.8 & 51865.9 & $\mid-14.1$ & -14.0 \\
\hline 11 & 362 & 110617 & 1128 & 480 & 180 & 51495.2 & 51.505 .5 & -10.3 & 51423.8 & 51437.9 & -14.1 & -35.7 \\
\hline 11 & 362 & 110625 & 1130 & 480 & 175 & 51233.8 & 51244.2 & -10.4 & 51108.0 & 51122.1 & -14.1 & -62.9 \\
\hline 11 & 362 & 110634 & 1132 & 480 & 170 & 2.6 & 51093.0 & -10.4 & 50787.0 & 50801.1 & $\mid-14.1$ & -147.8 \\
\hline 11 & 362 & 110644 & 1134 & 480 & 165 & 6.2 & 51156.7 & $|-10.5|$ & 51204.0 & 51218.1 & $|-14.1|$ & 28.9 \\
\hline 11 & 362 & 110654 & 1136 & 480 & 160 & 50944.6 & 50955.2 & $|-10.6|$ & 50452.4 & 50466.5 & $|-14.1|$ & -246.1 \\
\hline 11 & 362 & 110702 & 1138 & 480 & 155 & 51185.6 & 51196.2 & $|-10.6|$ & 50578.0 & 50592.1 & $\mid-14.1$ & -303.8 \\
\hline 11 & 36 & 110711 & 1140 & 480 & 150 & 52251.8 & 52262.5 & -10.7 & 52543.2 & 52557.2 & -14.0 & 145.7 \\
\hline 11 & 36 & 110720 & 1142 & 480 & 145 & 52985.4 & 52996.1 & -10.7 & 53794.2 & 53808.2 & $\mid-14.0$ & 404.4 \\
\hline 11 & $30<$ & 110729 & 1144 & 480 & 140 & 52714.0 & 52724.8 & -10.8 & 53115.4 & 53129.4 & -14.0 & 200.7 \\
\hline & 62 & 110737 & 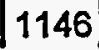 & 48 & 135 & 51892.6 & 51903.4 & -10.8 & 51809.2 & 51823.2 & -14 & -41.7 \\
\hline
\end{tabular}




\begin{tabular}{|c|c|c|c|c|c|c|c|c|c|c|c|c|}
\hline Line & Day & Time & Sta. & $\mathbf{x}$ & $\mathbf{Y}$ & uncorr. & $\begin{array}{l}\text { (nT) } \\
\text { corr. }\end{array}$ & | diff. & $\begin{array}{l}\text { BOT } \\
\text { uncorr. }\end{array}$ & $\begin{array}{l}\text { TOM }(n T) \\
\text { corr. }\end{array}$ & diff. & $\begin{array}{c}\text { Vert.Grad. } \\
\text { (nT/ft) }\end{array}$ \\
\hline 11 & 362 & 110745 & 1148 & 480 & 130 & 51001.0 & 51011.9 & -10.9 & 50553.0 & 50567.0 & -14.0 & -224.0 \\
\hline 11 & 362 & 110753 & 1150 & 480 & 125 & 50750.2 & 50761.1 & $|-10.9|$ & 50244.0 & 50258.0 & -14.0 & -253.1 \\
\hline 11 & 362 & 110802 & 1152 & 480 & 120 & 51207.2 & 51218.2 & $\mid-11.0$ & 50997.8 & 51011.8 & -14.0 & -104.7 \\
\hline 11 & 362 & 110810 & 1154 & 480 & 115 & 51660.8 & 51671.8 & -11.0 & 51489.4 & 51503.4 & -14.0 & -85.7 \\
\hline 11 & 362 & 110819 & 1156 & 480 & 110 & 52.023 .0 & 52034.1 & $|-11.1|$ & 51276.2 & 51290.2 & -14.0 & -373.4 \\
\hline 11 & 362 & 110827 & 1158 & 480 & 105 & 51859.8 & 51870.9 & $|-11.1|$ & 51004.0 & 51018.0 & -14.0 & -427.9 \\
\hline 11 & 362 & 110836 & 1160 & 480 & 100 & 51434.4 & 51445.5 & $|-11.1|$ & 50800.8 & 50814.8 & $\mid-14.0$ & -316.8 \\
\hline 11 & 362 & 110845 & 1162 & 480 & 95 & 51232.4 & 51243.6 & -11.2 & 50865.6 & 50879.6 & -14.0 & -183.4 \\
\hline 11 & 362 & 110854 & 1164 & 480 & 90 & 51201.4 & 51212.7 & $\mid-11.3$ & 51022.6 & 51036.6 & -14.0 & -89.4 \\
\hline 11 & 362 & 110901 & 1166 & 480 & 85 & 51216.4 & 51227.7 & $\mid-11.3$ & 51127.8 & 51141.8 & -14.0 & -44.3 \\
\hline 11 & 362 & 110909 & 1168 & 480 & 80 & 51230.0 & 51241.3 & -11.3 & 51180.8 & 51194.8 & -14.0 & -24.6 \\
\hline 11 & 362 & 110917 & 1170 & 480 & 75 & 51229.2 & 51240.6 & $\mid-11.4$ & 51203.6 & 51217.6 & -14.0 & -12.8 \\
\hline 11 & 362 & 110925 & 1172 & 480 & 70 & 51211.8 & 51223.2 & $|-11.4|$ & 51190.0 & 51204.0 & -14.0 & -10.9 \\
\hline 11 & 362 & 110934 & .1174 & 480 & 65 & 51165.6 & 51177.1 & $\mid-11.5$ & 51153.2 & 51167.2 & $\mid-14.0$ & -6.2 \\
\hline 11 & 362 & 110941 & 1176 & 480 & 60 & 075.4 & 51086.9 & $|-11.5|$ & 51052.0 & 066.0 & -14.0 & -11.7 \\
\hline 11 & 362 & 110949 & 1.178 & 480 & 55 & 50932.2 & 50943.8 & $\mid-11.6$ & 50857.6 & 50871.6 & $\mid-14.0$ & -37.3 \\
\hline 11 & 362 & 110958 & 1180 & 480 & 50 & 50749.8 & 50761.4 & $\mid-11.6$ & 50496.8 & 50510.8 & -14.0 & -126.5 \\
\hline 11 & 362 & 111006 & 1182 & 480 & 45 & 50709.8 & 50721.5 & $\mid-11.7$ & 50014.6 & 50028.6 & -14.0 & -347.6 \\
\hline 11 & 362 & 111015 & 1184 & 480 & 40 & 51545.6 & 51557.3 & $|-11.7|$ & 51012.6 & 51026.6 & -14.0 & -266.5 \\
\hline 11 & 362 & 111023 & 1186 & 480 & 35 & 52563.0 & 52574.8 & $\mid-11.8$ & 53396.4 & 53410.3 & $\mid-13.9$ & 416.7 \\
\hline 11 & 362 & 111032 & 1188 & 480 & 30 & 52602.4 & 52614.2 & $\mid-11.8$ & 53271.8 & 53285.7 & $|-13.9|$ & 334.7 \\
\hline 11 & 362 & 111040 & 1190 & 480 & 25 & 52205.4 & 52217.3 & -11.9 & 52428.0 & 52441.9 & -13.9 & 111.3 \\
\hline 11 & 362 & 111049 & 1192 & 480 & 20 & 51729.4 & 51741.3 & $\mid-11.9$ & 51670.2 & 51684.1 & -13.9 & -29.6 \\
\hline 11 & 362 & 111057 & 1194 & 480 & 15 & 51489.8 & 51501.8 & -12.0 & 51408.6 & 51422.5 & -13.9 & -40.6 \\
\hline 11 & 362 & 111105 & 1196 & 480 & 10 & 51417.6 & 51429.6 & $\mid-12.0$ & 51368.2 & 51382.1 & -13.9 & -24.7 \\
\hline 11 & 362 & 111114 & 1198 & 480 & 5 & 51385.8 & 51397.9 & $\mid-12.1$ & 51362.0 & 51375.9 & -13.9 & -11.9 \\
\hline 11 & 362 & 111122 & 1200 & 480 & 0 & 51390.6 & 51402.7 & $\mid-12.1$ & 5137.3 .2 & 51387.1 & -13.9 & -8.7 \\
\hline 12 & 362 & 111529 & 1212 & 470 & 0 & 51377.8 & 51390.3 & $|-12.5|$ & 51359.8 & 51375.4 & $\mid-15.6$ & -9.0 \\
\hline 12 & 362 & 111540 & 1214 & 470 & 5 & 51358.0 & 51370.7 & $\mid-12.7$ & 51326.4 & 51342.1 & $|-15.7|$ & -15.8 \\
\hline 12 & 362 & 111549 & 1216 & 470 & 10 & 51353.0 & 51365.7 & -12.7 & 51297.2 & 51313.0 & -15.8 & -27.9 \\
\hline 12 & 362 & 111558 & 1218 & 470 & 15 & 51380.0 & 51392.7 & -12.7 & 51309.2 & 51325.0 & -15.8 & -35.4 \\
\hline 12 & 362 & 111607 & 1220 & 470 & 20 & 51525.2 & 51537.9 & -12.7 & 51544.2 & 51560.1 & -15.9 & 9.5 \\
\hline 12 & 362 & 111617 & 1222 & 470 & 25 & 51732.4 & 51745.1 & $|-12.7|$ & 51972.6 & 51988.6 & $|-16.0|$ & 120.1 \\
\hline 12 & 362 & 111626 & 1224 & 470 & 30 & 51756.8 & 51769.5 & -12.7 & 52010.6 & 52026.7 & -16.1 & 126.9 \\
\hline 12 & 362 & 111634 & 1226 & 470 & 35 & $51687: 0$ & 51699.7 & $|-12.7|$ & 52221.4 & 52237.5 & $|-16.1|$ & 267.2 \\
\hline 12 & 362 & 111643 & 1228 & 470 & 40 & 51245.2 & 51258.0 & -12.8 & 51710.4 & 51726.6 & -16.2 & 232.6 \\
\hline 12 & 362 & 111651 & 1230 & 470 & 45 & 50732.0 & 50744.8 & $\mid-12.8$ & 50347.2 & 50363.5 & -16.3 & -192.4 \\
\hline 12 & 362 & 111659 & 1232 & 470 & 50 & 50767.6 & 50780.4 & -12.8 & 50573.8 & 50590.1 & -16.3 & -96.9 \\
\hline 12 & 362 & 111707 & 1234 & 470 & 55 & 50978.0 & 50990.8 & $|-12.8|$ & 50915.4 & 50931.8 & $|-16.4|$ & -31.3 \\
\hline 12 & 362 & 111716 & 1236 & 470 & 60 & 51105.8 & 51118.6 & $\mid-12.8$ & 51079.8 & 51096.3 & $\mid-16.5$ & -13.0 \\
\hline 12 & 362 & 111724 & 1238 & 470 & 65 & 51190.0 & 51202.8 & $\mid-12.8$ & 51168.8 & 51185.3 & $\mid-16.5$ & -10.6 \\
\hline 12 & 362 & 111732 & 1240 & 470 & 70 & 51230.0 & 51242.8 & $|-12.8|$ & 51206.2 & 51222.8 & $|-16.6|$ & -11.9 \\
\hline 12 & 362 & 111741 & 1242 & 470 & 75 & 51249.6 & 51262.4 & $\mid-12.8$ & 51216.2 & 51232.9 & $|-16.7|$ & -16.7 \\
\hline 12 & 362 & 111749 & 1244 & 470 & 80 & 51256.0 & 51268.9 & $\mid-12.9$ & 51207.0 & 51223.7 & -16.7 & -24.5 \\
\hline 12 & 362 & 111757 & 1246 & 470 & 85 & 51260.2 & 51273.1 & $\mid-12.9$ & 51188.0 & 51204.8 & -16.8 & -36.1 \\
\hline 12 & 362 & 111806 & 1248 & 470 & 90 & 51298.2 & 51311.1 & $\mid-12.9$ & 51184.2 & 51201.1 & $\mid-16.9$ & $-57: 0$ \\
\hline 12 & 362 & 111814 & 1250 & 470 & 95 & 51418.2 & 51431.1 & -12.9 & 51256.4 & 51273.3 & -16.9 & -80.9 \\
\hline 12 & 362 & 111823 & 1252 & 470 & 100 & 51772.2 & 51785.1 & $\mid-12.9$ & 51611.8 & 51628.8 & -17.0 & -80.2 \\
\hline 12 & 362 & 111831 & 1254 & 470 & 105 & 52614.4 & 52627.3 & -12.8 & 52677.0 & 52694.1 & $|-17.1|$ & 31.3 \\
\hline
\end{tabular}




\begin{tabular}{|c|c|c|c|c|c|c|c|c|c|c|c|c|}
\hline & & & & & & & $P(n T)$ & & BOT & TOM (nT) & & Vert.Grad. \\
\hline Line & Day & ime & ta. & $\mathrm{x}$ & $\mathbf{Y}$ & ncorr. & corr. & diff. & uncorr. & corr. & diff. & \\
\hline 12 & 362 & 111.841 & 1256 & 470 & 110 & 53692.0 & 53704.9 & -12.9 & 54620.6 & 54637.7 & -17.1 & 464.3 \\
\hline 12 & 362 & 111852 & 1258 & 470 & 115 & 767.8 & 53780.8 & -13.0 & 55311.6 & 55328.8 & -17.2 & 771.9 \\
\hline 12 & 362 & 111901 & 1260 & 470 & 120 & 2135.0 & 52148.0 & -13.0 & 51946.6 & .51963 .9 & $|-17.3|$ & -94.2 \\
\hline 12 & 362 & 111910 & 1262 & 470 & 125 & 51269.8 & 51282.8 & 13.0 & 50904.2 & 50921.6 & -17.4 & -182.8 \\
\hline 12 & 362 & 111918 & 1264 & |470| & 130 & 51411.2 & 1424.2 & $|-13.0|$ & 51063.2 & 1080.6 & $|-17.4|$ & -174.0 \\
\hline 12 & 362 & 27 & 1266 & 470 & 135 & 52075.6 & 2088.6 & $|-13.0|$ & 52047.4 & 2064.9| & -17.5 & -14.1 \\
\hline 12 & 362 & 136 & 1268 & 470 & 140 & 52953.0 & 52966.0 & $|-13.0|$ & 53408.0 & 53425.6 & -17.6 & 227.5 \\
\hline 12 & 362 & 45 & 1270 & 470 & 145 & 29.6 & 3142.6 & $|-13.0|$ & 54218.6 & 54236.2 & $|-17.6|$ & 544.5 \\
\hline 12 & 362 & 54 & 1272 & 470 & 150 & 5.2 & 52578.2 & 3.0 & 52722.0 & 2739.7 & $|-17.7|$ & 78.4 \\
\hline 12 & 362 & 112004 & 1274 & 470 & 155 & 628.4 & 51641.5 & -13.1 & 51271.6 & 1289.4 & $|-17.8|$ & -178.4 \\
\hline 12 & 362 & 112012 & 1276 & 470 & 160 & 616.2 & 51629.3 & 13.1 & 1639.0 & 556.9 & $|-17.9|$ & 11.4 \\
\hline 12 & 362 & 112022 & 1278 & 470 & 165 & 51789.2 & 51802,3 & -13.1 & 52149.6 & 52167.5 & -17.9 & 180.2 \\
\hline 12 & 362 & 112031 & 1280 & 470 & 170 & 51528.0 & 51541.1 & -13.1 & 51374.6 & 51392.6 & -18.0 & -76.7 \\
\hline 12 & 362 & 40 & 2 & 0 & 175 & 3.8 & 706.9 & $\mid-13.1$ & 24.4 & 42.5 & $|-18.1|$ & -84.7 \\
\hline 2 & 362 & 49 & 1284 & 470 & 180 & 52276.8 & 52289.9 & $|-13.1|$ & 52234.6 & 52252.7 & $\mid-18.1$ & -21.1 \\
\hline 12 & 362 & 57 & 1286 & 470 & 185 & 71.4 & 53184.5 & $|-13.1|$ & 53406.4 & 53424.6 & $-18.2 \mid$ & 117.5 \\
\hline 12 & 362 & 12106 & 1288 & 470 & 190 & 54060.8 & 54074.0 & -13.2 & 55141.0 & 55159.3 & -18.3 & 540.1 \\
\hline 12 & 362 & 112115 & 1290 & 470 & 195 & 54675.6 & 54688.8 & $|-13.2|$ & 55678.8 & 55697.2 & $|-18.4|$ & 501.6 \\
\hline 12 & 362 & 112123 & 1292 & 470이 & 200 & 54275.2 & 54288.4 & $\mid-13.2$ & 54915.2 & 4933.6 & $|-18.4|$ & 320.0 \\
\hline 12 & 362 & 32 & 1294 & 470 & 205 & 53720.8 & .53734 .0 & $\mid-13.2$ & 54803.2 & 4821.7| & $|-18.5|$ & 541.2 \\
\hline 12 & 362 & 40 & 1296 & 470 & 210 & 52554.8 & 52568.0 & $|-13.2|$ & 52420.6 & 2439.2 & $|-18.6|$ & -67.1 \\
\hline 12 & 362 & 49 & 1298 & 470 & 215 & 51558.8 & 51572.0 & $\mid-13.2$ & 51139.8 & 58.4 & $|-18.6|$ & -209.5 \\
\hline 12 & 362 & 58 & 1300 & 470 & 220 & 51247.4 & 51260.6 & $\mid-13.2$ & 51021.8 & |40.5 & $|-18.7|$ & -112.8 \\
\hline 12 & 362 & 206 & 1302 & $|470|$ & 225 & 51193.2 & 51206.5 & $|-13.3|$ & 51098.0 & 16.8 & $|-18.8|$ & -47.6 \\
\hline 12 & 362 & 112215 & 1304 & 470 & 230 & 51210.0 & 51223.3 & -13.3 & 51165.4 & $\mid 84.2$ & -18.8 & -22.3 \\
\hline 13 & 362 & 3 & 1306 & 460 & 230 & 51132.6 & 51146.4 & -13.8 & 51099.0 & 20.7 & $|-21.7|$ & -16.8 \\
\hline 13 & 362 & 20 & 1308 & 460 & 225 & 17.6 & 51131.4 & $\mid-13.8$ & 51049.6 & 71.3 & $|-21.7|$ & -34.0 \\
\hline 13 & 362 & 112830 & 1310 & 460 & 220 & 14.0 & 51127.9 & $|-13.9|$ & 50921.2 & 50943.0 & $|-21.8|$ & -96.4 \\
\hline 13 & 362 & 112839 & 1312 & 460 & 215 & 413.8 & 51427.7 & $|-13.9|$ & 50561.4 & 83.3 & $|-21.9|$ & $-426: 2$ \\
\hline 13 & 362 & 112848 & 1314 & 460 & 210 & 51741.8 & $|51755.7|$ & $\mid-13.9$ & 51315.2 & 337.2 & $|-22.0|$ & -213.3 \\
\hline 13 & 362 & 112858 & 1316 & 460 & 205 & 52672.4 & 52686.3 & $|-13.9|$ & 52335.2 & 52357.2 & $|-22.0|$ & -168.6 \\
\hline 13 & 362 & 112907 & 131,8 & 460 & 200 & 54163.0 & 54176.9 & -13.9 & 54461.4 & 54483.5 & $|-22.1|$ & 149.2 \\
\hline 13 & 362 & 112916 & 1320 & 460 & 195 & 55872.2 & 55886.1 & -13.9 & 19518.4 & 19540.6 & $|-22.2|$ & -18176.9 \\
\hline 13 & 362 & 112926 & 1322 & 460 & 190 & 56226.8 & 56240.7 & -13.9 & 35781.2 & 5803.5 & -22.3 & -10222.8 \\
\hline 13 & 362 & 1 & 24 & 460 & 18 & 5.8 & 79.8 & -14.0 & 08.0 & 930.3 & $|-22.3|$ & 521.1 \\
\hline 13 & 362 & 112944 & 26 & 460 & 180 & 53127.4 & $\mid 53141.4$ & $|-14.0|$ & 53245.8 & 3268.2 & $|-22.4|$ & 59.2 \\
\hline 13 & 362 & 112953 & 1328 & 460 & 175 & 51943.4 & $\mid 51957.4$ & $\mid-14.0$ & 51831.6 & 1854.1 & $|-22.5|$ & -55.9 \\
\hline 13 & 362 & 113002 & 1330 & |460| & $17 !$ & $51270.8^{\circ}$ & 51284.8 & -14.0 & 50813.2 & 50835.7 & $|-22.5|$ & -228.8 \\
\hline 13 & 362 & 113011 & 1332 & 460 & 165 & 51150.4 & 51164.4 & -14.0 & 51017.6 & 51040.2 & $|-22.6|$ & -66.4 \\
\hline 13 & 362 & 113019 & 1334 & 460 & 160 & 51043.0 & 51057.0 & $|-14.0|$ & 50677.8 & 3700.5 & $|-22.7|$ & -182.6 \\
\hline 13 & 362 & 113028 & 1336 & 460 & 155 & 51347.6 & $\mid 51361.6$ & $|-14.0|$ & 50703.6 & 0726.3 & $|-22.7|$ & -322.0 \\
\hline 13 & 362 & 113036 & 1338 & 460 & 150 & 52582.8 & 52596.9 & $|-14.1|$ & 53056.6 & 3079.4 & -22.8 & 236.9 \\
\hline 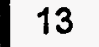 & 362 & 113046 & 1340 & 460 & 145 & 53384.0 & 53398.1 & $|-14.1|$ & 54468.0 & 54490.9 & $|-22.9|$ & 542.0 \\
\hline 13 & 362 & 113054 & 1342 & 460 & 140 & 53206.0 & 53220.1 & $\mid-14.1$ & 53971.0 & |53994.0| & $|-23.0|$ & 382.5 \\
\hline 13 & 362 & 113103 & 1344 & 460 & 135 & 52491.6 & 52505.7 & -14.1 & 52850.4 & 52873.4 & -23.0 & 179.4 \\
\hline 13 & 362 & 113111 & 1346 & 460 & 130 & 51598.8 & 51612.9 & $|-14.1|$ & 51335.8 & 51358.9 & $|-23.1|$ & -131.5 \\
\hline 11 & 362 & 113120 & 1348 & 460 & 125 & 336.8 & 51350.9 & -14.1 & 51103.2 & 51126.4 & $\mid-23.2$ & -116.8 \\
\hline 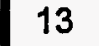 & 362 & 113129 & 1350 & 460 & 120 & 51561.6 & 51575.7 & $\mid-14.1$ & 51064.4 & 51087.6 & -23.2 & -248.6 \\
\hline & 002 & צונותו & 1352 & 460 & 115 & 52762.0 & $\mid 52776.1$ & | & 53195.2 & $|53218.5|$ & $\mid-23.3$ & 216.6 \\
\hline
\end{tabular}




\begin{tabular}{|c|c|c|c|c|c|c|c|c|c|c|c|c|}
\hline ne & Day & Time & a. & $x$ & $Y$ & & IT) & & $\mathbf{B C}$ & OM (nT) & if & Vert.Grad. \\
\hline 13 & & & & & & & & 1. & 1. & Trand & 11.. & \\
\hline 10 & $30<$ & 3740 & 54 & 400 & 770 & 759.6 & $1 / 13.8$ & 4.2 & 5634.0 & 55657.4 & |-23.4 & 37.2 \\
\hline 13 & 02 & 155 & 56 & 0 & & 90.0 & 52604.2 & .2 & 343.0 & 15966.4 & 23.4 & -18323.5 \\
\hline 13 & 362 & 113203 & 1358 & 460 & 100 & 51984.2 & 51998.4 & $\mid-14.2$ & 51975.4 & 51998.9 & -23.5 & -4.4 \\
\hline 13 & 362 & 113211 & 1360 & 460 & 95. & 51619.8 & 51634.0 & $\mid-14.2$ & 51558.6 & 51582.2 & -23.6 & -30.6 \\
\hline 13 & 362 & 113219 & 1362 & 460 & 90 & 51393.2 & 1407.4 & -14.2 & 1327.4 & 1351.0 & $\mid-23.6$ & -32.9 \\
\hline 13 & 362 & 113226 & 1364 & 460 & 85 & 51306.4 & 1320.6 & $\mid-14.2$ & 51263.4 & 51287.1 & $\mid-23.7$ & -21.5 \\
\hline 13 & 362 & 113234 & 1366 & 460 & 80 & 51284.8 & 51299.0 & $|-14.2|$ & 51249.0 & 51272.8 & $\mid-23.8$ & -17.9 \\
\hline 13 & 362 & 113242 & 1368 & 460 & 75 & 51277.2 & 51291.4 & $|-14.2|$ & 51250.0 & 51273.8 & $\mid-23.8$ & -13.6 \\
\hline 13 & 362 & 113250 & 1370 & 460 & 70 & 51261.8 & 1276.1 & $\mid-14.3$ & 51253.2 & 51277.1 & $\mid-23.9$ & -4.3 \\
\hline 13 & 362 & 113258 & 1372 & 460 & 65 & 51246.6 & 260.9 & $|-14.3|$ & 51239.0 & 1262.9 & $\mid-23.9$ & -3.8 \\
\hline 13 & 362 & 113306 & 1374 & 460 & 60 & 51205.6 & 219.9 & $|-14.3|$ & 51206.4 & 1230.4 & $\mid-24.0$ & 0.4 \\
\hline 13 & 362 & 113314 & 1376 & 460 & 55 & 24.2 & 138.5 & $|-14.3|$ & 1126.4 & 51150.5 & $\mid-24.1$ & 1.1 \\
\hline 13 & 362 & 113323 & 1378 & 460 & 50 & 50975.8 & 50990.1 & $|-14.3|$ & 50939.2 & 50963.3 & $\mid-24.1$ & -18.3 \\
\hline 13 & 362 & 113331 & 1380 & 460 & 45 & 50678.2 & 50692.5 & $|-14.3|$ & 50492.6 & 50516.8 & $\mid-24.2$ & -92.8 \\
\hline 13 & 362 & 113341 & 1382 & 460 & 40 & 50295.2 & 50309.5 & $\mid-14.3$ & 49576.8 & 49601.1 & $\mid-24.3$ & -359.2 \\
\hline 13 & 362 & 113348 & 1384 & 460 & 35 & 50290.8 & 50305.2 & $|-14.4|$ & 49102.8 & 49127.1 & $\mid-24.3$ & -594.0 \\
\hline 13 & 362 & 113357 & 1386 & 460 & 30 & 50416.4 & 50430.8 & $|-14.4|$ & -49770.4 & 49794.8 & $|-24.4|$ & -323.0 \\
\hline 13 & 362 & 113405 & 1388 & 460 & 25 & 50738.4 & 50752.8 & $|-14.4|$ & 50384.8 & 50409.3 & $\mid-24.5$ & -176.8 \\
\hline 13 & 362 & 113414 & 1390 & 460 & 20 & 51039.8 & 51054.2 & $|-14.4|$ & 50908.0 & 50932.5 & $|-24.5|$ & -65.9 \\
\hline 13 & 362 & 113422 & 1392 & 460 & 15 & 1.6 & 51226.0 & $\mid-14.4$ & 51143.0 & 51167.6 & $\mid-24.6$ & -34.3 \\
\hline 13 & 362 & 113430 & 1394 & 460 & 10 & 93.2 & 51307.6 & $|-14.4|$ & 51251.2 & 1275.9 & $|-24.7|$ & -21.0 \\
\hline 13 & 362 & 113437 & 1396 & 460 & 5 & 43.8 & 51358.2 & $|-14.4|$ & 51331.0 & 51355.7 & $|-24.7|$ & -6.4 \\
\hline 13 & 362 & 113445 & 1398 & 460 & 0 & 78.2 & 51392.6 & $|-14.4|$ & 51384.0 & 1408.8 & $\mid-24.8$ & 2.9 \\
\hline 14 & 362 & 113900 & 1410 & 450 & 0 & 51432.8 & 51448.5 & $|-15.7|$ & 51415.6 & 51440.4 & $\mid-24.8$ & -8.6 \\
\hline 14 & 362 & 13908 & 1412 & 450 & 5 & 1.4 & 51487.2 & -15.8 & 1433.4 & 1458.4 & -25.0 & -19.0 \\
\hline 14 & 362 & 113918 & 1414 & 450 & 10 & 76.6 & 51492.5 & $|-15.9|$ & 51426.4 & 1451.4 & $\mid-25.0$ & -25.1 \\
\hline 14 & 362 & 113926 & 1416 & 450 & 15 & 490.6 & 51506.5 & $|-15.9|$ & 51416.6 & 1441.6 & $\mid-25.0$ & -37.0 \\
\hline 14 & 362 & 113935 & 1418 & 450 & 20 & 3.6 & 51529.6 & -16.0 & 51463.0 & 51488.0 & $\mid-25.0$ & -25.3 \\
\hline 14 & 362 & 113944 & 1420 & 450 & 25 & 9.8 & 51535.9 & $|-16.1|$ & 51396.4 & 51421.4 & $\mid-25.0$ & -61.7 \\
\hline 14 & 362 & 113953 & 1422 & 450 & 30 & 35.2 & 51611.3 & $|-16.1|$ & 51530.4 & 1555.4 & $|-25.0|$ & -32.4 \\
\hline 14 & 362 & 114001 & 1424 & 450 & 35 & 5.6 & 51581.8 & $\mid-16.2$ & 51560.8 & 1585.8 & $|-25.0|$ & -2.4 \\
\hline 14 & 362 & 114010 & 1426 & 450 & 40 & 51466.6 & 51482.8 & $\mid-16.2$ & 51225.6 & 51250.6 & $\mid-25.0$ & -120.5 \\
\hline 14 & 362 & 114018 & 1428 & 450 & 45 & 51394.0 & 51410.3 & $|-16.3|$ & 51308.6 & 1333.6 & $|-25.0|$ & -42.7 \\
\hline 14 & 362 & 114025 & 1430 & 450 & 50 & 10.0 & 51356.3 & -16.3 & 51305.2 & 1330.2 & $|-25.0|$ & -17.4 \\
\hline 14 & 362 & 114033 & 1432 & $450^{\circ}$ & 55 & 51305.2 & 51321.5 & $|-16.3|$ & 51276.0 & 1301.0 & $|-25.0|$ & $-14.6^{\circ}$ \\
\hline 14 & 362 & 114041 & 1434 & 450 & 60 & 290.6 & 51307.0 & $|-16.4|$ & 51266.0 & 1291.0 & $|-25.0|$ & -12.3 \\
\hline 14 & 362 & 114049 & 1436 & 450 & 65 & 51286.6 & 51303.0 & $|-16.4|$ & 51264.8 & 51289.8 & $|-25.0|$ & -10.9 \\
\hline 14 & 362 & 114057 & 1438 & 450 & 70 & 20.4 & 51306.9 & $\mid-16.5$ & 51266,2 & $\mid 51291.2$ & $|-25.0|$ & -12.1 \\
\hline 14 & 362 & 114106 & 1440 & 450 & 75 & 4.6 & 51311.1 & $|-16.5|$ & 51266.2 & 1291.2 & $|-25.0|$ & -14.2 \\
\hline 14 & 362 & 114114 & 1442 & 450 & 80 & 33.2 & 51309.8 & $\mid-16.6$ & 51259.8 & 1284.8 & $|-25.0|$ & -16.7 \\
\hline 14 & 362 & 114123 & 1444 & 450 & 85 & 51298.4 & 51315.0 & $|-16.6|$ & 51245.4 & 51270.4 & $|-25.0|$ & -26.5 \\
\hline 14 & 362 & 114131 & 1446 & 450 & 90 & 299.4 & 51316.1 & $|-16.7|$ & 51219.2 & 51244.2 & $|-25.0|$ & -40.1 \\
\hline 14 & 362 & 114139 & 1448 & 450 & 95 & 51304.2 & 51320.9 & $|-16.7|$ & 51166.2 & 51191.2 & $|-25.0|$ & -69.0 \\
\hline 14 & 362 & 114147 & 1450 & 450 & 100 & 51357.2 & 51374.0 & -16.8 & 51172.8 & 51197.8 & $\mid-25.0$ & -92.2 \\
\hline 14 & 362 & 114155 & 1452 & 450 & 105 & 51635.4 & 51652.2 & -16.8 & 51604.4 & 51629.4 & -25.0 & -15.5 \\
\hline 14 & .362 & 114204 & 1454 & 450 & 110 & 52149.2 & 52166.1 & $|-16.9|$ & 52334.2 & 52359.2 & $\mid-25.0$ & 92.5 \\
\hline 14 & 362 & 114213 & 1456 & 450 & 115 & 52306.2 & 52323.1 & $|-16.9|$ & 52955.6 & 52980.6 & $\mid-25.0$ & 324.7 \\
\hline 14 & 362 & 114223 & 1458 & 450 & 120 & $\$ 10.8$ & 51627.8 & $\mid-17.0$ & 51279.8 & 51304.8 & -25.0 & -165.5 \\
\hline 14 & $362 \mid$ & $\mid 114232$ & 1460 & 450 & 125 & 51314.8 & 51331.9 & -17.1 & 50901.0 & 50926.0 & $\mid-25.0$ & -206.9 \\
\hline
\end{tabular}




\begin{tabular}{|c|c|c|c|c|c|c|c|c|c|c|c|c|}
\hline$n e$ & av & ime & a & $\lambda$ & $Y$ & & $\begin{array}{l}\mathrm{DP}(\mathrm{nT}) \\
\text { corr. }\end{array}$ & III. & $\begin{array}{l}\text { BOT } \\
\text { corr. }\end{array}$ & TOM (nT & | diff. & $\begin{array}{c}\text { Vert.Grad. } \\
\text { (nT/ft) }\end{array}$ \\
\hline 14 & 362 & 114241 & 1462 & 450 & 130 & 51509.2 & $\longdiv { 5 1 5 2 6 . 3 }$ & -17.1 & 51255.8 & 51280.8 & -25.0 & -126.7 \\
\hline 14 & 362 & 114250 & 1464 & 450 & 135 & 52087.0 & 52104.2 & $\mid-17.2$ & 52221.8 & 52246.8 & $|-25.0|$ & 67.4 \\
\hline 14 & 362 & 114300 & 1466 & 450 & 140 & 52598.0 & 52615.2 & $\mid-17.2$ & 53006.0 & 53031.0 & $|-25.0|$ & 204.0 \\
\hline 14 & 362 & 114309 & 1468 & 450 & 145 & 52562.8 & 52580.1 & $\mid-17.3$ & 52878.0 & 52902.9 & $|-24.9|$ & 157.6 \\
\hline 14 & 362 & 114318 & 1470 & 450 & 150 & 51769.0 & 51786.3 & -17.3 & 51685.8 & 51710.7 & -24.9 & -41.6 \\
\hline 14 & 362 & 114327 & 1472 & 450 & 155 & 50693.8 & 50711.2 & -17.4 & 50178.4 & 50203.3 & -24.9 & -257.7 \\
\hline 14 & 362 & 114336 & 1474 & 450 & 160 & 50371.0 & 50388.4 & -17.4 & 49791.6 & 49816.5 & -24.9 & -289.7 \\
\hline 14 & 362 & 114345 & $1476^{\prime}$ & 450 & 165 & 50736.2 & 50753.7 & -17.5 & 50616.4 & 50641.3 & -24.9 & -59.9 \\
\hline 14 & 362 & $114353^{\circ}$ & 1478 & 450 & 170 & 50960.0 & 50977.5 & -17.5 & 50560.4 & 50585.3 & -24.9 & -199.8 \\
\hline 14 & 362 & 114403 & 1480 & 450 & 175 & 51514.8 & 51532.4 & -17.6 & 51354.2 & 51379.1 & -24.9 & -80.3 \\
\hline 14 & 362 & 114412 & 1482 & 450 & 180 & 52420.2 & 52437.9 & -17.7 & 52344.4 & 52369.3 & -24.9 & -37.9 \\
\hline 14 & 362 & 114421 & 1484 & 450 & 185 & 53838.4 & 53856.1 & -17.7 & 54370.6 & 54395.5 & -24.9 & 266.1 \\
\hline 14 & 362 & 114429 & 1486 & 450 & 190 & 55385.6 & 55403.4 & $|-17.8|$ & 57742.8 & 57767.7 & $|-24.9|$ & 1178.6 \\
\hline 14 & 362 & 114438 & 1488 & 450 & 195 & 55082.2 & 55100.0 & -17.8 & 55901.8 & 55926.7 & -24.9 & 409.8 \\
\hline 14 & 362 & 114447 & 1490 & 450 & 200 & 101.0 & 54118.9 & -17.9 & 54622.2 & 4647.1 & -24.9 & 260.6 \\
\hline 14 & 362 & 114456 & 1492 & 450 & 205 & 53269.6 & 53287.5 & -17.9 & 53873.0 & 53897.9 & -24.9 & 301.7 \\
\hline 14 & 362 & 114505 & 1494 & 450 & 210 & 52260.0 & 52278.0 & -18.0 & 52488.6 & 52513.5 & -24.9 & 114.3 \\
\hline 14 & 362 & 114513 & 1496 & 450 & $215^{\prime}$ & 51412.0 & 51430.0 & $|-18.0|$ & 51258.8 & 51283.7 & $|-24.9|$ & -76.6 \\
\hline 14 & 362 & 114522 & 1498 & 450 & 220 & 51074.2 & 51092.3 & $|-18.1|$ & 50905.0 & 50929.9 & $|-24.9|$ & -84.6 \\
\hline 14 & 362 & 114530 & 1500 & $450^{\circ}$ & 225 & 51017.2 & 51035.3 & -18.1 & 50914.2 & 50939.1 & -24.9 & -51.5 \\
\hline 14 & 362 & 114538 & 1502 & 450 & 230 & 51065.4 & 51083.6 & -18.2 & 51007.8 & 51032.7 & $\mid-24.9$ & -28.8 \\
\hline 15 & 362 & 114900 & 1504 & 440 & 230 & 50961.4 & 50980.8 & -19.4 & 50925.4 & 50950.2 & -24.8 & -18.0 \\
\hline 15 & 362 & 114909 & 1506 & 440 & 225 & 50841.6 & 50861.0 & -19.4 & 50760.4 & 50785.2 & $-24,8$ & -40.6 \\
\hline 15 & 362 & 114918 & 1508 & 440 & 220 & 50716.2 & 50735.7 & -19.5 & 50537.4 & 50562.2 & -24.8 & -89.4 \\
\hline 15 & 362 & 114926 & 1510 & 440 & $215^{\circ}$ & 50744.0 & 50763.5 & -19.5 & 50395.6 & 50420.4 & -24.8 & -174.2 \\
\hline 15 & 362 & 114935 & 1512 & 440 & 210 & 51234.0 & 51253.6 & $-19,6$ & 51113.0 & 51137.8 & -24.8 & -60.5 \\
\hline 15 & 362 & 114943 & 1514 & 440 & 205 & 51987.8 & 52007.4 & $|-19.6|$ & 52716.8 & 52741.6 & $|-24.8|$ & 364.5 \\
\hline 15 & 362 & 114951 & 1516 & 440 & 200 & 52085.4 & 52105.1 & -19.7 & 52209.0 & 52233.8 & -24.8 & 61.8 \\
\hline 15 & 362 & 115000 & 1518 & 440 & 195 & 52203.4 & 52223.1 & -19.7 & 52166.2 & 52191.0 & -24.8 & -18.6 \\
\hline 15 & 362 & 115008 & 1520 & 440 & 190 & 52306.8 & 52326.6 & -19.8 & 52579.4 & 52604.2 & -24.8 & 136.3 \\
\hline 15 & 362 & 115016 & 1522 & 440 & 185 & 52029.0 & 52048.8 & -19.8 & 52081.2 & 52106.0 & -24.8 & 26.1 \\
\hline 15 & 362 & 115024 & 1524 & 440 & 180 & 51580.2 & 51600.1 & -19.9 & 51563.2 & 51588.0 & -24.8 & -8.5 \\
\hline 15 & 362 & 115033 & 1526 & 440 & 175 & 51102.2 & 51122.1 & -19.9 & 50926.2 & 50951.0 & -24.8 & -88.0 \\
\hline 15 & 362 & 115042 & 1528 & 440 & 170 & 50750.0 & 50770.0 & -20.0 & 50272.2 & 50297.0 & -24.8 & -238.9 \\
\hline 15 & 362 & 115050 & 1530 & 440 & 165 & 50653.2 & 50673.2 & -20.0 & 50564.2 & 50589.0 & -24.8 & -44.5 \\
\hline 15 & 362 & 115058 & 1532 & 440 & 160 & 50230.6 & 50250.7 & -20.1 & 49756.6 & 49781.4 & -24.8 & -237.0 \\
\hline 15 & 362 & 115107 & 1534 & 440 & 155 & 50113.6 & 50133.7 & $|-20.1|$ & 52058.4 & 52083.2 & $\mid-24.8$ & 972.4 \\
\hline 15 & 362 & 115115 & 1536 & 440 & 150 & 51041.6 & 51061.8 & $\mid-20.2$ & 52064.0 & 52088.8 & $\mid-24.8$ & 511.2 \\
\hline 15 & 362 & 115124 & 1538 & 440 & 145 & 52532.6 & 52552.8 & $\mid-20.2$ & 52477.0 & 52501.8 & $|-24.8|$ & -27.8 \\
\hline 15 & 362 & 115132 & 1540 & 440 & 140 & 52849.2 & 52869.5 & -20.3 & 53758.4 & 53783.2 & -24.8 & 454.6 \\
\hline 15 & 362 & 115140 & 1542 & 440 & 135 & 52179.2 & 52199.5 & -20.3 & 52243.6 & 52268.4 & -24.8 & 32.2 \\
\hline 15 & 36 & 115149 & 1544 & 440 & 130 & 51542.0 & 51562.4 & $\mid-20.4$ & 51320.4 & 51345.2 & $|-24.8|$ & -110.8 \\
\hline 15 & 362 & 115158 & 1546 & 440 & 125 & 51559.8 & 51580.2 & $\mid-20.4$ & 51510.0 & 51534.8 & $|-24.8|$ & -24.9 \\
\hline 15 & 362 & 115206 & 1548 & 440 & 120 & 51860.0 & 51880.5 & -20.5 & 52022.6 & 52047.4 & -24.8 & 81.3 \\
\hline 15 & 362 & 115215 & 1550 & 440 & 115 & 52181.6 & 52202.1 & -20.5 & 52849.6 & 52874.4 & -24.8 & 334.0 \\
\hline 15 & 362 & 115223 & 1552 & 440 & 110 & 51746.0 & 51766.6 & -20.6 & 51451.8 & 51476.6 & -24.8 & -147.1 \\
\hline 15 & 362 & 115232 & 1554 & 440 & 105 & 51478.0 & 51498.6 & -20.6 & 51091.0 & 51115.8 & -24.8 & -193.5 \\
\hline 15 & 362 & 115240 & 1556 & 440 & 100 & 51395.0 & 51415.7 & -20.7 & 51047.0 & 51071.8 & -24.8 & -174.0 \\
\hline 15 & 362 & 115248 & 1558 & $|440|$ & 95 & 51383.0 & 51403.7 & $\mid-20.7$ & 51280.4 & 51305.2 & -24.8 & -51.3 \\
\hline
\end{tabular}




\begin{tabular}{|c|c|c|c|c|c|c|c|c|c|c|c|c|}
\hline & & & & & & & & & & OM $(n T$ & & \\
\hline Line & Day & Time & Sta. & $\mathbf{X}$ & $\mathbf{Y}$ & uncorr. & corr. & diff. & incorr. & corr. & diff. & \\
\hline 15 & 362 & 115255 & 1560 & 440 & 90 . & 51362.0 & 51382.8 & -20.8 & 51308.0 & 51332.8 & -24.8 & -27.0 \\
\hline 15 & 362 & 115304 & 1562 & 440 & 85 & 327.8 & 51348.6 & -20.8 & 51301.8 & 51326.6 & -24.8 & -13.0 \\
\hline 15 & 362 & 115312 & 1564 & 440 & 80 & 11.4 & 51332.3 & -20.9 & 51286.2 & 1311.0 & $\mid-24.8$ & -12.6 \\
\hline 15 & 362 & 115320 & 1566 & 440 & 75 & 89.2 & 51310.1 & -20.9 & 51276.0 & 1300.8 & $\mid-24.8$ & -6.6 \\
\hline 15 & 362 & 115328 & 1568 & 440 & 70 & 278.2 & 51299.2 & -21.0 & 51255.0 & 1279.7 & $|-24.7|$ & -11.6 \\
\hline 15 & 362 & 115336 & 1570 & 440 & 65 & 275.0 & 51296.0 & -21.0 & 1245.0 & 1269.7 & $|-24.7|$ & -15.0 \\
\hline 15 & 362 & 115344 & 1572 & 440 & 60 & 276.4 & 51297.5 & -21.1 & 1243.6 & 1268.3 & -24.7 & -16.4 \\
\hline 15 & 362 & 115351 & 1574 & 440 & 55 & 21.0 & 1342.1 & -21.1 & 1251.0 & 1275.7 & -24.7 & -35.0 \\
\hline 15 & 362 & 115400 & 1576 & 440 & 50 & 01.2 & 522.4 & -21.2 & 383.8 & 408.5 & $|-24.7|$ & -58.7 \\
\hline 15 & 362 & 115408 & 1578 & 440 & 45 & 75.6 & 2096.8 & -21.2 & 954.0 & |978.7 & $|-24.7|$ & -60.8 \\
\hline 15 & 362 & 115416 & 1580 & 440 & 40 & 158.0 & 3179.3 & -21.3 & 3541.2 & 3565.9 & -24.7 & 191.6 \\
\hline 15 & 362 & 115424 & 1582 & 440 & 35 & .4 & 53736.7 & -21.3 & 54465.4 & $\$ 490.1$ & $\mid-24.7$ & 375.0 \\
\hline 5 & 362 & 132 & 1584 & 440 & 30 & 7.0 & 38.4 & -21.4 & 752.6 & 777.3 & -24.7 & 167.8 \\
\hline 15 & 362 & 141 & 1586 & 440 & 25 & 3.0 & 2977.4 & -21.4 & 19.6 & 244.3 & $|-24.7|$ & 131.8 \\
\hline 15 & 362 & 50 & 1588 & 440 & $20^{\circ}$ & 0.0 & 491.5 & -21.5 & 57.0 & 581.7 & $-24.7 \mid$ & 43.5 \\
\hline 15 & 362 & 159 & 1590 & 440 & 15 & .2 & 2058.7 & -21.5 & 2026.4 & 2051.1 & -24.7 & -5.4 \\
\hline 15 & 362 & 115507 & 1592 & 440 & 10 & 748.4 & 1770.0 & -21.6 & 51721.0 & 1745.7 & -24.7 & -13.7 \\
\hline 15 & 362 & 115515 & 1594 & 440 & 5 & 51584.0 & 51605.6 & -21.6 & 51568.6 & 1593.3 & -24.7 & -7.7 \\
\hline 15 & 362 & $\$ 22$ & 1596 & 440 & 0 & .8 & 23.5 & -21.7 & 89.8 & 4.5 & -24.7 & -6.0 \\
\hline 16 & 362 & 336 & 1618 & 430 & 0 & & 66.8 & -3.2 & 0.8 & 3.0 & -12.2 & -16.4 \\
\hline 16 & 362 & 351 & 1620 & 430 & 5 & & 6.1 & -3.1 & 3.8 & 1.0 & $\mid-12.2$ & -32.1 \\
\hline 16 & 362 & 300 & 1622 & 430 & 10 & & 38.1 & -3.1 & 07.2 & 9.4 & -12.2 & -28.9 \\
\hline 16 & 362 & 308 & 1624 & 430 & 15 & .2 & 11.3 & -3.1 & 36.2 & 88.3 & -12.1 & 39.0 \\
\hline 16 & 362 & 131920 & 1626 & 430 & 20 & .8 & 387.9 & -3.1 & 53827.0 & 3839.1 & -12.1 & 221.1 \\
\hline 16 & 362 & 13 & 1628 & 430 & 25 & & 5.1 & -3.1 & 25.8 . & 37.9 & $|-12.1|$ & 526.9 \\
\hline 16 & 362 & 13 & 1630 & 430 & 30 & & 4.7 & -3.1 & 8.2 & 70.3 & $\mid-12.1$ & 508.3 \\
\hline 18 & 362 & 13 & 1632 & 430 & 35 & .8 & 5.9 & -3.1 & 2.4 & 4.5 & $\mid-12.1$ & 16525.2 \\
\hline 16 & 362 & 13 & 1634 & 430 & 40 & & 8.9 & -3.1 & 49.8 & 561.9 & -12.1 & 59.0 \\
\hline 16 & 362 & 132000 & 1636 & 430 & 45 & & & -3.1 & 51266.2 & 278.2 & -12.0 & -273.3 \\
\hline 16 & 362 & 132008 & 1638 & 430 & 50 & & 46.9 & -3.1 & 51071.8 & 083.8 & $|-12.0|$ & -136.0 \\
\hline 16 & 362 & 132015 & 1640 & 430 & 55 & & 239.3 & -3.1 & 51112.2 & 124.2 & $|-12.0|$ & -62.0 \\
\hline 16 & 362 & 132023 & 1642 & 430 & 60 & 4.8 & 227.9 & -3.1 & 162.2 & 174.2 & $|-12.0|$ & -31.3 \\
\hline 16 & 362 & 132031 & 1644 & 430 & 65 & & 46.1 & -3.1 & 05.4 & 17.4 & $|-12.0|$ & -18.8 \\
\hline 16 & 362 & 132038 & 1646 & 430 & 70 & $: 0$ & 0.1 & -3.1 & & 3.2 & $|-12.0|$ & -12.9 \\
\hline 16 & 362 & 132046 & 1648 & 430 & $7 !$ & & & -3.1 & 0.2 & 2.1 & $\mid-11.9$ & -13.3 \\
\hline 16 & 362 & 132054 & 1650 & 430 & 80 & & 7.2 & -3.0 & 7.4 & & -77.9 & -14.9 \\
\hline 16 & 362 & 132102 & 1652 & 430 & 85 & 7.8 & 0.8 & -3.0 & 3.6 & & -11.9 & -14.6 \\
\hline 16 & 362 & 132111 & 1654 & 430 & 90 & 4.0 & 57.0 & -3.0 & 9.6 & & $\mid-11.9$ & -22.2 \\
\hline  & 362 & 132118 & 1656 & 430 & 95 & & 92.8 & -3.0 & 51765.4 & 777.3 & -11.9 & -12.2 \\
\hline 16 & 362 & 132242 & 1658 & 430 & 100 & 17.4 & 2220.4 & -3.0 & 52354.6 & 366.3 & -11.7 & 68.6 \\
\hline 16 & 362 & 132250 & 1660 & 430 & 10 & & 396.2 & -3.0 & 1.2 & 12.9 & -77.6 & 104.0 \\
\hline 16 & 36 & 132259 & 1662 & 430 & 11 & & 48.5 & -2.9 & 29.6 & 241.3 & $\mid-11.7$ & -8.0 \\
\hline 16 & 362 & 132308 & 1664 & 430 & 11 & & 14.5 & -2.9 & 3.6 & 85.3 & -11.7 & -19.0 \\
\hline 76 & 362 & 132316 & 1666 & 430 & 12 & 0.8 & 349.7 & -2.9 & 08.4 & 20.0 & -11.6 & -119.2 \\
\hline & 362 & 132324 & 1668 & 430 & 12 & 0.4 & 9.3 & -2.9 & 35.4 & 747.0 & $\mid-11.6$ & -220.5 \\
\hline 16 & 362 & 132332 & 1670 & 430 & 13 & 6.2 & 49.1 & -2.9 & 50.4 & 2.0 & $\mid-11.6$ & -147.9 \\
\hline 16 & 362 & 132341 & 1672 & 430 & $13 !$ & 41.8 & 52244.7 & -2.9 & 90.4 & .0 & $\mid-11.6$ & 74.3 \\
\hline 16 & 362 & 132349 & 1674 & 430 & 140 & 52932.4 & 52935.3 & -2.9 & 54074.8 & 4086.4 & -11.6 & 571.2 \\
\hline 16 & 362 & 132356 & 1676 & 430 & 145 & 52962.4 & 52965.3 & -2.9 & 54164.8 & 54176.4 & -11.6 & 601.2 \\
\hline
\end{tabular}




\begin{tabular}{|c|c|c|c|c|c|c|c|c|c|c|c|c|}
\hline & & & & & & & (nT) & & $\mathbf{B}$ & TON & & \\
\hline ine & Day & Time & Sta. & $\mathbf{x}$ & $\mathbf{Y}$ & uncorr. & corr. & diff. & uncorr. & corr. & diff. & \\
\hline 16 & 362 & 132403 & 1678 & 430 & 150 & 51733.0 & 51735.9 & -2.9 & 51815.6 & 51827.1 & -11.5 & 41.3 \\
\hline 16 & 362 & 132412 & 1680 & 430 & 155 & 50723.2 & 50726.1 & -2.9 & 50325.6 & 50337.1 & -11.5 & -198.8 \\
\hline 16 & 362 & 132419 & 1682 & 430 & 160 & 50674.2 & 50677.1 & -2.9 & 50586.6 & 50598.1 & -11.5 & -43.8 \\
\hline 16 & 362 & 132427 & 1684 & 430 & 165 & 50711.4 & 50714.3 & -2.9 & 50584.6 & 50596.1 & $\mid-11.5$ & -63.4 \\
\hline 16 & 362 & 132435 & 1686 & 430 & 170 & 50607.2 & 50610.1 & -2.9 & 50164.8 & 50176.3 & -11.5 & -221.2 \\
\hline 16 & 362 & 132443 & 1688 & 430 & 175 & 50834.6 & 50837.5 & -2.9 & 50698.4 & 50709.9 & -11.5 & -68.1 \\
\hline 16 & 362 & 132450 & 1690 & 430 & 180 & 51096.4 & 51099.3 & -2.9 & 50969.0 & 50980.5 & -11.5 & -63.7 \\
\hline 16 & 362 & 132458 & 1692 & $430 \mid$ & 185 & 51324.2 & 51327.0 & -2.8 & 51253.2 & 51264.6 & $|-11.4|$ & -35.5 \\
\hline 16 & 362 & 132506 & 1694 & 430 & 190 & 51386.2 & 51389.0 & -2.8 & 51513.6 & 51525.0 & $\mid-11.4$ & 63.7 \\
\hline 16 & 362 & 132515 & 1696 & 430 & 195 & 50861.8 & 50864.6 & -2.8 & 50496.6 & 50508.0 & -11.4 & -182.6 \\
\hline 16 & 362 & 132523 & 1698 & 430 & 200 & 50328.8 & 50331.6 & -2.8 & 49600.4 & 49611.8 & $\mid-11.4$ & -364.2 \\
\hline 16 & 362 & 132531 & 1700 & $|430|$ & 205 & 50100.0 & 50102.8 & -2.8 & 49398.2 & 49409.6 & -11.4 & -350.9 \\
\hline 16 & 362 & 132538 & 1702 & 430 & 210 & 50086.0 & 50088.8 & -2.8 & 49548.6 & 49560.0 & -11.4 & -268.7 \\
\hline 16 & 362 & 132547 & 1704 & 430 & 215 & 50265.8 & 50268.6 & -2.8 & 49920.4 & 49931.7 & -11.3 & -172.7 \\
\hline 16 & 362 & 132555 & 1706 & 430 & 220 & 50554.8 & 50557.6 & -2.8 & 50412.6 & 50423.9 & -11.3 & -71.1 \\
\hline 16 & 362 & 132602 & 1708 & 430 & 225 & 50788.8 & 50791.6 & -2.8 & 50738.4 & 50749.7 & -11.3 & -25.2 \\
\hline 16 & 362 & 132610 & 1710 & 430 & 230 & 50952.4 & 50955.2 & -2.8 & 50929.0 & 50940.3 & -11.3 & -11.7 \\
\hline 17 & 362 & 132901 & 1712 & 420 & 230 & 50918.6 & 50921.2 & -2.6 & 50923.6 & 50934.5 & $\mid-10.9$ & 2.5 \\
\hline 17 & 362 & 132909 & 1714 & 420 & 225 & 50820.4 & 50823.0 & -2.6 & 50821.6 & 50832.5 & -10.9 & 0.6 \\
\hline 17 & 362 & 132917 & 1716 & 420 & 220 & 50607.0 & 50609.6 & -2.6 & 50553.4 & 50564.3 & -10.9 & -26.8 \\
\hline 17 & 362 & 132925 & 1718 & 420 & 215 & 50363.2 & 50365.8 & -2.6 & 50143.6 & 50154.5 & $\mid-10.9$ & -109.8 \\
\hline 17 & 362 & 132933 & 1720 & 420 & 210 & 50267.2 & 50269.8 & -2.6 & 49951.4 & 49962.3 & $\mid-10.9$ & -157.9 \\
\hline 17 & 362 & 132941 & 1722 & 420 & 205 & 50457.0 & 50459.6 & -2.6 & 50416.4 & 50427.3 & $\mid-10.9$ & -20.3 \\
\hline 17 & 362 & 132949 & 1724 & 420 & 200 & 50777.4 & 50780.0 & -2.6 & 50576.8 & 50587.7 & -10.9 & -100.3 \\
\hline 17 & 362 & 132958 & 1726 & 420 & 195 & 51351.6 & 51354.2 & -2.6 & 51515.4 & 51526.2 & $\mid-10.8$ & 81.9 \\
\hline 17 & 362 & 133006 & 1728 & 420 & 190 & 51776.4 & 51779.0 & -2.6 & 52310.0 & 52320.8 & -10.8 & 266.8 \\
\hline $17^{\prime}$ & 362 & 133014 & 1730 & 420 & 185 & 51510.8 & 51513.4 & -2.6 & 51691.2 & 51702.0 & -10.8 & 90.2 \\
\hline 17 & 362 & $\mid 133022$ & 1732 & 420 & 180 & 51134.4 & 51137.0 & -2.6 & 51117.2 & 51128.0 & $\mid-10.8$ & -8.6 \\
\hline 17 & 362 & 133030 & 1734 & 420 & 175 & 50841.6 & 50844.2 & -2.6 & 50737.2 & 50748.0 & -10.8 & -52.2 \\
\hline 17 & 362 & 133037 & 1736 & 420 & 170 & 50692.0 & 50694.6 & -2.6 & 50383.4 & 50394.2 & -10.8 & -154.3 \\
\hline 17 & 362 & 133046 & 1738 & 420 & 165 & 50951.8 & 50954.4 & -2.6 & 51175.8 & 51186.5 & -10.7 & 112.0 \\
\hline 17 & 362 & 133055 & 1740 & 420 & 160 & 50783.4 & 50786.0 & -2.6 & 50811.4 & 50822.1 & -10.7 & 14.0 \\
\hline 17 & 362 & 133102 & 1742 & 420 & 155 & 50607.8 & 50610.3 & -2.5 & 50116.0 & 50126.7 & $|-10.7|$ & -245.9 \\
\hline 17 & 362 & 133111 & 1744 & 420 & 150 & 51265.6 & 51268.1 & -2.5 & .51017 .2 & 51027.9 & -10.7 & -124.2 \\
\hline 17 & 362 & 133119 & 1746 & 420 & 145 & 52225.6 & 52228.1 & -2.5 & 52537.6 & 52548.3 & $|-10.7|$ & 156.0 \\
\hline 17 & 362 & 133128 & 1748 & 420 & 140 & 52405.4 & 52407.9 & -2.5 & 52801.4 & 52812.1 & -10.7 & 198.0 \\
\hline 17 & 362 & 133136 & 1750 & 420 & 135 & 51826.2 & 51828.7 & -2.5 & 51757.8 & 51768.4 & $\mid-10.6$ & -34.2 \\
\hline 17 & 362 & 133144 & 1752 & 420 & 130 & $51271.6^{\circ}$ & 51274.1 & -2.5 & 50966.2 & 50976.8 & -10.6 & -152.7 \\
\hline 17 & 362 & 133153 & 1754 & 420 & 125 & 51088.2 & 51090.7 & -2.5 & 50711.2 & 50721.8 & $\mid-10.6$ & -188.5 \\
\hline 17 & 362 & 133201 & 17,56 & 420 & 120 & 51324.2 & 51326.7 & -2.5 & 50976.4 & 50987.0 & $\mid-10: 6$ & -173.9 \\
\hline 17 & 362 & 133210 & 1758 & 420 & 115 & 51842.0 & 51844.5 & -2.5 & 51795.0 & 51805.6 & -10.6 & -23.5 \\
\hline 17 & 362 & 133218 & 1760 & 420 & 110 & 52410.2 & 52412.7 & -2.5 & 52604.8 & 52615.4 & -10.6 & 97.3 \\
\hline 17 & 362 & 133227 & 1762 & 420 & 105 & 52815.2 & 52817.7 & -2.5 & 53610.4 & 53620.9 & -10.5 & 397.6 \\
\hline 17 & 362 & 133235 & 1764 & 420 & 100 & 52481.6 & 52484.1 & -2.5 & 52759.4 & 52769.9 & $\mid-10.5$ & 138.9 \\
\hline 17 & 362 & 133242 & 1766 & 420 & 95 & 51968.4 & 51970.9 & -2.5 & 51992.4 & 52002.9 & $|-10.5|$ & 12.0 \\
\hline 17 & 362 & 133250 & 1768 & 420 & 90 & 51655.6 & 51658.1 & -2.5 & 51630.2 & 51640.7 & $\mid-10.5$ & -12.7 \\
\hline 17 & 362 & 133258 & 1770 & 420 & 85 & 51482.8 & 51485.3 & -2.5 & 51469.0 & 51479.5 & $\mid-10.5$ & -6.9 . \\
\hline 17 & 362 & 133306 & 1772 & 420 & 80 & 51374.0 & 51376.4 & -2.4 & 51362.6 & 51373.1 & -10.5 & -5.7 \\
\hline 17 & 362 & $|133314|$ & 1774 & 420 & 75 & 51294.0 & 51296.4 & -2.4 & 51280.0 & 51290.4 & -10.4 & -7.0 \\
\hline
\end{tabular}




\begin{tabular}{|c|c|c|c|c|c|c|c|c|c|c|c|c|}
\hline Line & Day & Time & Sta. & $\mathbf{x}$ & $\mathbf{Y}$ & uncorr. & $\begin{array}{l}\text { DP (nT) } \\
\text { corr. }\end{array}$ & diff. & $\begin{array}{r}\text { BO7 } \\
\text { uncorr. }\end{array}$ & $\begin{array}{l}\text { TOM (nt } \\
\text { corr. }\end{array}$ & I diff. & $\begin{array}{c}\text { Vert.Grad. } \\
\text { (nT/ft) }\end{array}$ \\
\hline 17 & 362 & 133321 & 1776 & 420 & 70 & 51229.8 & 51232.2 & -2.4 & 51214.8 & 51225.2 & -10.4 & -7.5 \\
\hline 17 & 362 & 133329 & 1778 & 420 & 65 & 51168.0 & 51170.4 & -2.4 & 51131.8 & 51142.2 & -10.4 & -18.1 \\
\hline 17 & 362 & 133337 & 1780 & 420 & 60 & 51104.0 & 51106.4 & -2.4 & 51035.6 & 51046.0 & -10.4 & -34.2 \\
\hline 17 & 362 & 133345 & 1782 & 420 & 55 & 51049.6 & 51052.0 & -2.4 & 50917.2 & 50927.6 & $\mid-10.4$ & -66.2 \\
\hline 17 & 362 & 133353 & 1784 & 420 & 50 & 51132.4 & 51134.8 & -2.4 & 50850.8 & 50861.2 & $|-10.4|$ & -140.8 \\
\hline 17 & 362 & 133401 & 1786 & 420 & 45 & 51744.0 & 51746.4 & -2.4 & .51427 .2 & 51.437 .5 & $|-10.3|$ & -158.4 \\
\hline 17 & 362 & 133409 & 1788 & 420 & 40 & 53164.2 & 53166.6 & -2.4 & 24410.6 & 24420.9 & -10.3 & -14376.8 \\
\hline 17 & 362 & 133418 & 1790 & 420 & 35 & 54549.4 & 54551.8 & -2.4 & 55343.6 & 55353.9 & -10.3 & 397.1 \\
\hline 17 & 362 & 133426 & 1792 & 420 & 30 & .54960 .8 & 9963.2 & -2.4 & 56072.6 & 56082.9 & $|-10.3|$ & 555.9 \\
\hline 17 & 362 & 133435 & 1794 & 420 & 25 & 54175.4 & 4177.8 & -2.4 & 54315.6 & 54325.9 & $\mid-10.3$ & 70.1 \\
\hline 17 & 362 & 133443 & 1796 & 420 & 20 & 53171.4 & 53173.8 & -2.4 & 53175.6 & 53185.9 & $\mid-10.3$ & 2.1 \\
\hline 17 & 362 & 133451 & 1798 & 420 & 15 & 52318.4 & 52320.8 & -2.4 & 52153.0 & 52163.2 & $\mid-10.2$ & -82.7 \\
\hline 17 & 362 & 133459 & 1800 & 420 & 10 & 51826.2 & 1828.6 & -2.4 & 51695.6 & 51705.8 & $\mid-10.2$ & -65.3 \\
\hline 17 & 362 & 133509 & 1802 & 420 & 5 & 51613.4 & 1615.7 & -2.3 & 51531.8 & 51542.0 & $\mid-10.2$ & -40.8 \\
\hline 17 & 362 & 133517 & 1804 & 420 & 0 & 51516.2 & 1518.5 & -2.3 & 51456.0 & 51466.2 & -10.2 & -30.1 \\
\hline 18 & 362 & 133953 & 1816 & 410 & 0 & 51405.8 & 407.0 & -1.2 & 51318.2 & 51328.3 & $|-10.1|$ & -43.8 \\
\hline 18 & 362 & 134005 & 1818 & 410 & 5 & 51477.8 & 1478.9 & -1.1 & 51344.4 & 51354.4 & -10.0 & -66.7 \\
\hline 18 & 362 & 134014 & 1820 & 410 & 10 & 51743.4 & $i 1744.5$ & -1.1 & 51563.2 & 51573.2 & $\mid-10.0$ & -90.1 \\
\hline 18 & 362 & 134023 & 1822 & 410 & 15 & 52298.6 & 52299.6 & -1.0 & 52183.4 & 52193.4 & $\mid-10.0$ & -57.6 \\
\hline 18 & $362^{\circ}$ & 134031 & 1824 & 410 & 20 & 53470.4 & 53471.4 & -1.0 & 53874.8 & 53884.8 & $\mid-10.0$ & 202.2 \\
\hline 18 & 362 & 134039 & 1826 & 410 & 25 & 54858.0 & 54858.9 & -0.9 & 56144.2 & 56154.2 & $\mid-10.0$ & 643.1 \\
\hline 18 & 362 & 134046 & 1828 & 410 & 30 & 54535.8 & 54536.7 & -0.9 & 20961.4 & 20971.3 & $\mid-9.9$ & -16787.2 \\
\hline 18 & 362 & 134054 & 1830 & 410 & 35 & 52930.4 & 52931.3 & -0.9 & 52971.8 & 52981.7 & -9.9 & 20.7 \\
\hline 18 & 362 & 134101 & 1832 & 410 & 40 & 51779.0 & 51779.8 & -0.8 & 52053.0 & $52062: 9$ & -9.9 & 137.0 \\
\hline 18 & 362 & 134110 & 1834 & 410 & 45 & 50898.0 & 50898.8 & -0.8 & 50531.6 & 50541.5 & $\mid-9.9$ & -183.2 \\
\hline 18 & 362 & 134118 & 1836 & 410 & 50 & 50688.2 & 50689.0 & -0.8 & 50368.2 & 50378.1 & $|-9.9|$ & -160.0 \\
\hline 18 & 362 & 134126 & 1838 & 410 & .55 & 50821.8 & 50822.5 & -0.7 & 50680.2 & 506900.1 & -9.9 & -70.8 \\
\hline 18 & 362 & 134133 & 1840 & 410 & 60 & 50981.0 & 50981.7 & -0.7 & 50918.2 & 50928.1 & -9.9 & -31.4 \\
\hline 18 & 362 & 134141 & 1842 & 410 & 65 & 51097.4 & 51098.0 & -0.6 & 51064.0 & 510073.9 & -9.9 & -16.7 \\
\hline 18 & 362 & 134148 & 1844 & 410 & 70 & 51200.2 & 51200.8 & -0.6 & 51167.4 & 51177.3 & -9.9 & -16.4 \\
\hline 18 & 362 & 134155 & 1846 & 410 & 75 & 51276:0 & 51276.6 & -0.6 & 51252.4 & 51262.3 & -9.9 & -11.8 \\
\hline 18 & 362 & 134202 & 1848 & 410 & 80 & 51368.2 & 51368.7 & -0.5 & 51339.8 & 51349.7 & -9.9 & -14.2 \\
\hline 18 & 362 & 134210 & 1850 & 410 & 85 & 51500.0 & 51500.5 & -0.5 & 51461.4 & 51471.3 & $|-9.9|$ & -19.3 \\
\hline 18 & 362 & 134218 & 1852 & 410 & 90 & 51708.8 & 51709.3 & -0.5 & 51666.6 & 51676.5 & $\mid-9.9$ & -21.1 \\
\hline 18 & 362 & 134226 & 1854 & 410 & 95 & 52034.2 & 52034.6 & -0.4 & 52035.6 & 52045.5 & -9.9 & 0.7 \\
\hline 18 & 362 & 134234 & 1856 & 410 & 100 & 52458.8 & 52459.2 & -0.4 & 52587.8 & 52597.7 & -9.9 & 64.5. \\
\hline 18 & 362 & 134243 & 1858 & 410 & 105 & 52733.8 & 52734.1 & -0.3 & 53101.2 & 53111.1 & $\mid-9.9$ & 183.7 \\
\hline 18 & 362 & 134251 & $1860 \mid$ & 410 & 110 & 52526.0 & 52526.3 & -0.3 & 52556.8 & 52566.7 & $\mid-9.9$ & 15.4 \\
\hline 18 & 362 & 134259 & 1862 & 410 & 115 & 52322.4 & 52322.7 & -0.3 & 52369.8 & 52379.7 & $\mid-9.9$ & 23.7 \\
\hline 18 & 362 & 134309 & 1864 & 410 & 120 & 51959.2 & 51959.4 & -0.2 & 51650.6 & 51660.5 & $\mid-9.9$ & -154.3 \\
\hline 18 & 362 & 134318 & 1866 & 410 & 125 & 51533.4 & 51533.6 & -0.2 & 51055.0 & 51064.9 & -9.9 & -239.2 \\
\hline 18 & 362 & 134326 & 1868 & 410 & 130 & 51539.0 & 51539.1 & -0.1 & 51216.2 & 51226.1 & -9.9 & -161.4 \\
\hline 18 & 362 & 134334 & 1870 & 410 & 135 & 52005.4 & 52005.5 & -0.1 & 52107.2 & 52117.1 & $\mid-9.9$ & 50.9 \\
\hline 18 & 362 & 134342 & 1872 & 410 & 140 & 52308.8 & 52308.9 & -0.1 & 52964.8 & 52974.7 & -9.9 & 328.0 \\
\hline 18 & 362 & 134350 & 1874 & 410 & 145 & 51571.8 & 51571.8 & 0.0 & 51316.2 & 51326.0 & $\mid-9.8$ & -127.8 \\
\hline 18 & 362 & 134358 & 1876 & 410 & 150 & 50634.2 & 50634.2 & 0.0 & 49697.6 & 49707.4 & -9.8 & -468.3 \\
\hline 18 & 362 & 134406 & 1878 & 410 & 155 & 50288.4 & 50288.3 & 0.1 & 49565.0 & 49574.8 & -9.8 & -361.7 \\
\hline 18 & 362 & 134415 & 1880 & 410 & 160 & 50582.8 & 50582.7 & 0.1 & .50322 .6 & 50332.4 & -9.8 & -130.1 \\
\hline 18 & 362 & 134422 & 1882 & 410 & 165 & 50901.4 & 50901.3 & 0.1 & 50955.0 & 50964.8 & -9.8 & 26.8 \\
\hline
\end{tabular}




\begin{tabular}{|c|c|c|c|c|c|c|c|c|c|c|c|c|}
\hline & & & & & & & (nT) & & BO1 & TOM (nT & & \\
\hline Line & Day & Time & Sta. & $X$ & $Y$ & uncorr. & corr. & diff. & uncorr. & corr. & diff. & it) \\
\hline 18 & 362 & 134430 & 1884 & 410 & 170 & 50758.0 & 50757.8 & 0.2 & 50463.6 & 50473.4 & -9.8 & -147.2 \\
\hline 18 & 362 & 134438 & 1886 & 410 & 175 & 50825.0 & 50824.8 & 0.2 & 50687.6 & 50697.4 & -9.8 & -68.7 \\
\hline 18 & 362 & 134445 & 1888 & 410 & 180 & 51123.4 & 51123.1 & 0.3 & 51088.2 & 51098.0 & -9.8 & -17.6 \\
\hline 18 & 362 & 134452 & 1890 & 410 & 185 & 51493.4 & 51493.1 & 0.3 & 51556.8 & 51566.6 & -9.8 & 31.7 \\
\hline 18 & 362 & 134459 & 1892 & 410 & 190 & 51728.2 & 51727.9 & 0.3 & 51982.8 & 51992.6 & -9.8 & 127.3 \\
\hline 18 & 362 & 134506 & 1894 & 410 & 195 & 51553.6 & 51553.2 & 0.4 & 51624.2 & 51634.0 & -9.8 & 35.3 \\
\hline 18 & 362 & 134514 & 1896 & 410 & 200 & 51215.8 & 51215.4 & 0.4 & 51177.4 & 51187.2 & -9.8 & -19.2 \\
\hline 18 & 362 & 134522 & 1898 & 410 & 205 & 50873.6 & 50873.2 & 0.4 & 50742.6 & 0752.4 & -9.8 & -65.5 \\
\hline 18 & 362 & 134530 & 1900 & 410 & 210 & 50590.0 & 50589.5 & 0.5 & 50335.4 & 0345.2 & -9.8 & -127.3 \\
\hline 18 & 362 & 134537 & 1902 & 410 & 215 & 50605.2 & .50604 .7 & 0.5 & 50469.8 & 50479.6 & -9.8 & -67.7 \\
\hline 18 & 362 & 134545 & 1904 & 410 & 220 & 50760.8 & 50760.3 & 0.5 & 50717.4 & 50727.2 & -9.8 & -21.7 \\
\hline 18 & 362 & 134552 & 1906 & 410 & 225 & 50909.8 & 50909.2 & 0.6 & 50900.6 & 50910.4 & -9.8 & -4.6 \\
\hline 18 & 362 & 134600 & 1908 & 410 & 230 & 51027.4 & 51026.8 & 0.6 & 51029.4 & 51039.2 & -9.8 & 1.0 \\
\hline 19 & 362 & 135257. & 1910 & 400 & 230 & 012.4 & 51009.7 & 2.7 & 51012.4 & 1022.0 & -9.6 & 0.0 \\
\hline 19 & 362 & 135306 & 1912 & 400 & 225 & 90.6 & 87.9 & 2.7 & 50881.6 & 891.1 & -9.5 & -4.5 \\
\hline 19 & 362 & 135314 & 1914 & 400 & 220 & 50727.8 & 50725.1 & 2.7 & 50678.2 & 687.7 & -9.5 & -24.8 \\
\hline 19 & 362 & 135323 & 1916 & 400 & 215 & 50608.0 & 50605.2 & 2.8 & 50472.4 & 481.9 & -9.5 & -67.8 \\
\hline 19 & 362 & 135330 & 1918 & 400 & 210 & 50768.6 & 50765.8 & 2.8 & 50675.8 & 50685.3 & -9.5 & -46.4 \\
\hline 19 & 362 & 135338 & 1920 & 400 & 205 & 51345.0 & 51342.1 & 2.9 & 51698.4 & 51707.9 & -9.5 & 176.7 \\
\hline 19 & 362 & 135346 & 1922 & 400 & 200 & 52021.0 & 52018.1 & 2.9 & 52784.8 & 52794.3 & -9.5 & 381.9 \\
\hline 19. & 362 & 135354 & 1924 & 400 & 195 & 52194.2 & 52191.3 & 2.9 & 52923.8 & 52933.3 & -9.5 & 364.8 \\
\hline 19 & 362 & 135402 & 1926 & 400 & 190 & 51895.4 & 51892.4 & 3.0 & 52238.0 & 52247.5 & -9.5 & 171.3 \\
\hline 19 & 362 & 135424 & 1928 & 400 & 185 & $51342.0^{\prime}$ & 51338.9 & 3.1 & 51379.6 & $51389: 1$ & -9.5 & 18.8 \\
\hline 19 & 362 & 135431 & 1930 & 400 & 180 & 50956.2 & 50953.1 & 3.1 & 50858.0 & 50867.5 & -9.5 & -49.1 \\
\hline 19 & 362 & 135438 & 1932 & 400 & 175 & 50718.8 & 50715.7 & 3.1 & 50515.6 & 50525.1 & -9.5 & -101.6 \\
\hline 19 & 362 & 135446 & 1934 & 400 & 170 & 50672.6 & 50669.4 & 3.2 & 50302.4 & 50311.9 & -9.5 & -185.1 \\
\hline 19 & 362 & 135455 & 1936 & 400 & 165 & 51089.0 & 51085.8 & 3.2 & 51307.0 & 51316.5 & -9.5 & 109.0 \\
\hline 19 & 362 & 135503 & 1938 & 400 & 160 & 99.4 . & 50996.1 & 3.3 & 50905.8 & 50915.3 & -9.5 & -46.8 \\
\hline 19 & 362 & 135511 & 1940 & 400 & 155 & 94.2 & 50990.9 & 3.3 & 50399.4 & 50408.9 & -9.5 & -297.4 \\
\hline 19 & 362 & 135518 & 1942 & 400 & 150 & 5.6 & 51772.3 & 3.3 & 51747.8 & 51757.3 & -9.5 & -13.9 \\
\hline 19 & 362 & 135527 & 1944 & 400 & $\uparrow 45$ & 52595.2 & 52591.8 & 3.4 & 52962.0 & 371.5 & -9.5 & 183.4 \\
\hline 19 & 362 & 135534 & 1946 & 400 & 140 & 52893.2 & 52889.8 & 3.4 & 53615.2 & 53624.7 & -9.5 & 361.0 \\
\hline 19 & 362 & 135542 & 1948 & 400 & 135 & 52308.4 & 52304.9 & 3.5 & 52262.8 & 52272.3 & -9.5 & -22.8 \\
\hline 19 & 362 & 135550 & 1950 & 400 & 130 & 51964.0 & 51960.5 & 3.5 & 51356.8 & 51366.3 & -9.5 & -303.6 \\
\hline 19 & 362 & 135559 & 1952 & 400 & 125 & 53656.8 & 53653.3 & 3.5 & 35049.8 & 35059.3 & -9.5 & -9303.5 \\
\hline 19 & 362 & 135607 & 1954 & 400 & 120 & 54880.0 & 54876.4 & 3.6 & 52179.6 & 52189.0 & -9.4 & -1350.2 \\
\hline 19 & 362 & 135614 & 1956 & 400 & 115 & 34.6 & 53281.0 & 3.6 & 53448.8 & 53458.2 & -9.4 & 82.1 \\
\hline 19 & 362 & 135622 & 1958 & 400 & 110 & 3.0 & 39.3 & 3.7 & 53046.4 & 055.8 & -9.4 & 101.7 \\
\hline 19 & 362 & 135630 & 1960 & 400 & 105. & 6.6 & 52902.9 & 3.7 & 53566.8 & 53576.2 & -9.4 & 330.1 \\
\hline 19 & 362 & 135638 & 1962 & 400 & 100 & 34.6 & 52480.9 & 3.7 & 52771.8 & $|52781.2|$ & -9.4 & 143.6 \\
\hline 19 & 362 & 135645 & 1964 & 400 & 95 & 51956.0 & 51952.2 & 3.8 & 52001.0 & 2010.4 & -9.4 & 22.5 \\
\hline 19 & 362 & 135653 & 1966 & 400 & 90 & 51620.0 & 51616.2 & 3.8 & 51585.8 & 51595.2 & -9.4 & -17.1 \\
\hline 19 & 362 & 135701 & 1968 & 400 & 85 & 51419.6 & 51415.8 & 3.8 & 51385.6 & 51395.0 & -9.4 & -17.0 \\
\hline 19 & 362 & 135709 & 1970 & 400 & 80 & 51310.0 & 51306.1 & 3.9 & 51280.2 & 51289.6 & $-9.4^{\circ}$ & -14.9 \\
\hline 19 & 362 & 135717 & 1972 & 400 & 75 & 20.4 & 51216.5 & 3.9 & 51213.6 & 51223.0 & -9.4 & -3.4 \\
\hline 19 & 362 & 135724 & 1974 & 400 & 70 & 51148.4 & 51144.4 & 4.0 & 51144.2 & $\mid 51153.6$ & -9.4 & -2.1 \\
\hline 19 & 362 & 135732 & 1976 & 400 & 65 & 51053.8 & 51049.8 & 4.0 & 51044.0 & 51053.4 & -9.4 & -4.9 \\
\hline 19 & 362 & 135740 & 1978 & 400 & 60 & 50911.6 & 50907.6 & 4.0 & 50883.2 & 50892.6 & -9.4 & -14.2 \\
\hline 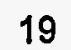 & 362 & 135748 & 1980 & 400 & 55 & 50673.0 & 50668.9 & 4.1 & 50591.6 & 50601.0 & -9.4 & -40.7 \\
\hline
\end{tabular}




\begin{tabular}{|c|c|c|c|c|c|c|c|c|c|c|c|c|}
\hline & & & & & & & $O D \ln T$ & & - & TTOR $\mathrm{AnT}$ & & \\
\hline Line & |Day & ime & Sta. & $\mathbf{x}$ & $\mathbf{Y}$ & uncorr. & corr. & diff. & uncor & corr. & diff.' & \\
\hline 19 & 362 & 135756 & 1982 & 400 & 50 & 50235.6 & 50231.5 & 4.1 & 49981.6 & 49991.0 & -9.4 & -127.0 \\
\hline 19 & 362 & 135805 & 1984 & 400 & 45 & 49881.2 & 49877.0 & 4.2 & 48961.6 & 48971.0 & -9.4 & -459.8 \\
\hline 19 & 362 & 135813 & 1986 & 400 & 40 & 49846.8 & 49842.6 & 4.2 & 48444.0 & 48453.4 & -9.4 & -701.4 \\
\hline 19 & 362 & 135822 & 1988 & 400 & 35 & 51373.2 & 51369.0 & $4: 2$ & 51164.4 & 51173.8 & -9.4 & -104.4 \\
\hline 19 & 362 & 135830 & 1990 & $400 \mid$ & 30 & 53063.4 & 53059.1 & 4.3 & 54612.0 & 54621.4 & -9.4 & 774.3 \\
\hline 19 & 362 & 135838 & 1992 & 400 & 25 & 52974.0 & 52969.7 & 4.3 & 53832.0 & $\mid 53841.4$ & -9.4 & 429.0 \\
\hline 19 & 362 & 135848 & 1994 & 400 & 20 & 52364.0 & 52359.6 & 4.4 & 52386.8 & 52396.2 & -9.4 & 11.4 \\
\hline 19 & 362 & 135859 & 1996 & $400 \mid$ & 15 & 51574.0 & 51569.6 & 4.4 & 51157.8 & 51167.2 & -9.4 & -208.1 \\
\hline 19 & 362 & 135911 & 1998 & |400| & 10 & 51320.4 & 51315.9 & 4.5 & 51062.8 & 51072.1 & -9.3 & -128.8 \\
\hline 19 & 362 & 135927 & 2000 & 400 & 5 & 51304.8 & 51300.2 & 4.6 & 51152.2 & 51161.5 & -9.3 & -76.3 \\
\hline 19 & 362 & 135943 & 2002 & 400 & 0 & 51329.2 & 51324.6 & 4.6 & 51249.0 & 51258.3 & -9.3 & -40.1 \\
\hline 20 & 362 & 140601 & 2016 & 390 & 0 & 51350.8 & 51345.2 & 5.6 & 51294:0 & 51302.4 & -8.4 & -28.4 \\
\hline 20 & 362 & 140609 & 2018 & 390 & 5 & 51342.2 & 51336.4 & 5.8 & 51250.8 & 51259.1 & -8.3 & -45.7 \\
\hline 20 & 362 & 140617 & 2020 & 390 & 10 & 51385.6 & 51379.8 & 5.8 & 51236.8 & 51245.1 & -8.3 & -74.4 \\
\hline 20 & 362 & 140626 & 2022 & $|390|$ & 15 & 51537.4 & 51531.5 & 5.9 & 51302.0 & 51310.3 & $-8.3^{\prime}$ & -117.7 \\
\hline 20 & 362 & 140634 & 2024 & 390 & $20^{\circ}$ & 51927.8 & 51921.9 & 5.9 & 51824.2 & 51832.5 & -8.3 & -51.8 \\
\hline 20 & 362 & 140644 & 2026 & 390 & 25 & 52142.8 & 52136.9 & 5.9 & 52347.2 & 52355.4 & -8.2 & 102.2 \\
\hline 20 & 362 & 140652 & 2028 & 390 & 30 & 51644.4 & 51638.5 & 5.9 & 51865.0 & 51873.2 & -8.2 & 110.3 \\
\hline 20 & 362 & 140700 & 2030 & 390 & 35 & 50438.8 & 50432.8 & 6.0 & 49769.4 & 49777.6 & -8.2 & -334.7 \\
\hline 20 & 362 & 140709 & 2032 & 390 & 40 & 49276.4 & 49270.4 & 6.0 & 45616.4 & 45624.6 & -8.2 & -1830.0 \\
\hline 20 & 362 & 140718 & 2034 & 390 & 45 & 49759.2 & 49753.2 & 6.0 & 49098.4 & 49106.5 & -8.1 & -330.4 \\
\hline 20 & 362 & 140734 & 2036 & 390 & 50 & 50465.8 & 50459.7 & 6.1 & 50378.4 & 50386.5 & -8.1 & -43.7 \\
\hline 20 & 362 & 140743 & 2038 & 390 & 55 & 50800.4 & 50794.3 & 6.1 & 50789.8 & 50797.9 & -8.1 & -5.3 \\
\hline 20 & 362 & 140751 & 2040 & 390 & 60 & 50981.2 & 50975.1 & 6.1 & 50971.4 & 50979.4 & -8.0 & -4.9 \\
\hline 20 & 362 & 140759 & 2042 & 390 & 65 & 51080.0 & 51073.9 & 6.1 & 51072.6 & 51080.6 & -8.0 & -3.7 \\
\hline 20 & 362 & 140808 & 2044 & 390 & 70 & 51142.6 & 51136.5 & 6.1 & 51126.4 & 51134.4 & -8.0 & -8.1 \\
\hline 20 & 362 & 140816 & 2046 & 390 & 75 & 51184.0 & 51177.8 & 6.2 & 51163.6 & 51171.6 & -8.0 & -10.2 \\
\hline 20 & 362 & 140824 & 2048 & 390 & 80 & 51225.8 & 51219.6 & 6.2 & 51192.8 & 51200.7 & -7.9 & -16.5 \\
\hline 20 & 362 & 140832 & 2050 & 390 & 85 & 51283.0 & 51276.8 & 6.2 & 51233.2 & 51241.1 & -7.9 & -24.9 \\
\hline 20 & 362 & 140840 & 2052 & 390 & 90 & 51402.2 & 51396.0 & 6.2 & 51341.4 & 51349.3 & -7.9 & -30.4 \\
\hline 20 & 362 & 140848 & 2054 & $390 \mid$ & 95 & 51637.6 & 51631.3 & 6.3 & 51644.4 & 51652.3 & -7.9 & 3.4 \\
\hline 20 & 362 & 140857 & 2056 & 390 & 100 & 52055.4 & 52049.1 & 6.3 & 52379.6 & 52387.4 & -7.8 & 162.1 \\
\hline 20 & 362 & 140906 & 2058 & 390 & 105 & 52233.2 & 52226.9 & 6.3 & 53099.6 & 53107.4 & -7.8 & 433.2 \\
\hline 20 & 362 & 140914 & 2060 & 390 & 110 & 51429.4 & 51423.1 & 6.3 & 51128.8 & 51136.6 & -7.8 & -150.3 \\
\hline 20 & 362 & 140922 & 2062 & 390 & 115 & 51046.6 & 51040.2 & 6.4 & 50049.0 & 50056.8 & -7.8 & -498.8 \\
\hline 20 & 362 & 140930 & 2064 & 390 & 120 & 51489.4 & 51483.0 & 6.4 & 52276.6 & 52284.3 & -7.7 & 393.6 \\
\hline 20 & 362 & 140939 & 2066 & 390 & 125 & 50887.4 & 50881.0 & 6.4 & 49937.2 & 49944.9 & -7.7 & -475.1 \\
\hline 20 & 362 & 140947 & 2068 & $390 \mid$ & 130 & 51277.6 & 51271.2 & 6.4 & 50534.2 & 50541.9 & -7.7 & -371.7 \\
\hline 20 & 362 & 140955 & 2070 & 390 & 135 & 52720.8 & 5271.4 .4 & 6.4 & 52832.6 & 52840.3 & -7.7 & 55.9 \\
\hline 20 & 362 & 141003 & 2072 & 390 & 140 & 53395.0 & 53388.5 & 6.5 & 54449.0 & 54456.7 & -7.7 & 527.0 \\
\hline 20 & 362 & 141012 & 2074 & $390 \mid$ & 145 & 53197.6 & 53191.1 & 6.5 & 53705.8 & 53713.4 & -7.6 & 254.1 \\
\hline 20 & 362 & 141020 & 2076 & 390 & 150 & 52427.8 & 52421.3 & 6.5 & 52544.8 & 52552.4 & -7.6 & 58.5 \\
\hline 20 & 362 & 141028 & 2078 & 390 & 155 & 51702.6 & 51696.1 & 6.5 & 51605.2 & 51612.8 & -7.6 & -48.7 \\
\hline 20 & 362 & 141042 & 2080 & 390 & 160 & 51160.6 & 51154.0 & 6.6 & 50793.0 & 50800.5 & -7.5 & -183.8 \\
\hline 20 & 362 & 141051 & 2082 & $390 \mid$ & 165 & 50728.4 & 50721.8 & 6.6 & 50255.2 & 50262.7 & -7.5 & -236.6 \\
\hline 20 & 362 & 141059 & 2084 & 390 & 170 & 50490.6 & 50484.0 & 6.6 & 50085.8 & 50093.3 & -7.5 & -202.4 \\
\hline 20 & 362 & 141108 & 2086 & $390 \mid$ & 175 & $50586: 2$ & 50579.6 & 6.6 & 50394.2 & 50401.7 & -7.5 & -96.0 \\
\hline 20 & 362 & 141117 & 2088 & $390 \mid$ & 180 & 50797.4 & 50790.7 & 6.7 & 50659.2 & 50666.6 & -7.4 & -69.1 \\
\hline 20 & 362 & 141125 & 2090 & $390 \mid$ & 185 & 51118.8 & 51112.1 & 6.7 & 51097.2 & 51104.6 & -7.4 & -10.8 \\
\hline
\end{tabular}




\begin{tabular}{|c|c|c|c|c|c|c|c|c|c|c|c|c|}
\hline & & & & & & & (nT) & & BO7 & TOM (nT & & \\
\hline Line & Day & Time & Sta. & $x$ & $\mathbf{Y}$ & uncorr. & corr. & diff. & uncorr. & corr. & diff. & \\
\hline 20 & 362 & 141134 & 2092 & 390 & 190 & 51604.6 & 51597.9 & 6.7 & 51928.2 & 51935.6 & -7.4 & 161.8 \\
\hline 20 & 362 & 1143 & 2094 & 390 & 195 & 51580.6 & 51573.9 & 6.7 & 51926.0 & 51933.4 & -7.4 & 172.7 \\
\hline 20 & 362 & 1152 & 2096 & 390 & 200 & 50719.6 & 50712.8 & 6.8 & 50360.8 & 50368.1 & -7.3 & -179.4 \\
\hline 20 & 362 & 141200 & 2098 & 390 & 205 & 49993.8 & 49987.0 & 6.8 & 49085.0 & 49092.3 & -7.3 & -454.4 \\
\hline 20 & 362 & 41207 & 2100 & 390 & 210 & 49828.2 & 49821.4 & 6.8 & 49061.2 & 49068.5 & 7.3 & -383.5 \\
\hline 20 & 362 & 141215 & 2102 & 390 & $215^{\circ}$ & 50282.0 & 50275.2 & 6.8 & 50059.2 & 50066.5 & -7.3 & -111.4 \\
\hline 20 & 362 & 141222 & 2104 & $390^{\circ}$ & 220 & 50652.2 & 50645.4 & 6.8 & 50592.2 & 50599.4 & -7.2 & -30.0 \\
\hline 20 & 362 & 41230 & 2106 & 390 & 225 & 50891.0 & 50884.1 & 6.9 & 50881.8 & 50889.0 & -7.2 & -4.6 \\
\hline 20 & 362 & 41238 & 2108 & 390 & 230 & 51058.6 & 51051.7 & 6.9 & 51061.0 & 51068.2 & -7.2 & 1.2 \\
\hline 21 & 362 & 41544 & 2110 & 380 & 230 & 51078.4 & 51071.0 & 7.4 & 51106.8 & 51113.5 & -6.7 & 14.2 \\
\hline 21 & 362 & 141553 & 2112 & 380 & 225 & 50966.0 & 50958.6 & 7.4 & 50970.8 & 50977.4 & -6.6 & 2.4 \\
\hline 21 & 362 & 141601 & 2114 & 380 & 220 & 50801.4 & |50793.9 & 7.5 & 50762.8 & 50769.4 & -6.6 & -19.3 \\
\hline 21 & 362 & 141608 & 2116 & 380 & 215 & 50624.8 & 50617.3 & 7.5 & 50522.2 & 50528.8 & -6.6 & -51.3 \\
\hline 21 & 362 & 141617 & 2118 & 380 & 210 & 50472.0 & 50464.5 & 7.5 & 50274.8 & 50281.4 & -6.6 & -98.6 \\
\hline 21 & 362 & 41625 & 2120 & 380 & 205 & 50676.8 & 50669.3 & 7.5 & 50561.6 & 50568.1 & -6.5 & -57.6 \\
\hline 21 & 362 & 141635 & 2122 & 380 & 200 & 51041.6 & 51034.1 & 7.5 & 50916.8 & 50923.3 & -6.5 & -62.4 \\
\hline 21 & 362 & 141646 & 2124 & 380 & 195 & 51148.8 & 51141.2 & 7.6 & 50997.8 & 51004.3 & -6.5 & -75.5 \\
\hline 21 & 362 & 141657 & 2126 & 380 & 190 & 51091.4 & 51083.8 & 7.6 & 51042.8 & 51049.2 & -6.4 & -24.3 \\
\hline 21 & 362 & 141705 & 2128 & 380 & 185 & 50839.2 & 50831.6 & 7.6 & 50722.8 & 50729.2 & -6.4 & -58.2 \\
\hline 21 & 362 & 141714 & 2130 & 380 & 180 & 50620.8 & 50613.1 & 7.7 & 50439.2 & 50445.6 & -6.4 & -90.8 \\
\hline 21 & 362 & 141723 & 2132 & 380 & 175 & 50452.0 & 50444.3 & 7.7 & 50200.6 & 50207.0 & -6.4 & -125.7 \\
\hline 21 & 362 & 141731 & 2134 & 380 & 170 & 50513.6 & 50505.9 & 7.7 & 49795.6 & 49801.9 & -6.3 & -359.0 \\
\hline 21 & 362 & 141739 & 2136 & 380 & 165 & 50956.4 & 50948.7 & 7.7 & 50772.0 & 50778.3 & -6.3 & -92.2 \\
\hline 21 & 362 & 141747 & 2138 & 380 & 160 & 51428.6 & 51420.9 & 7.7 & 51217.2 & 51223.5 & -6.3 & -105.7 \\
\hline 21 & 362 & 141756 & 2140 & 380 & 155 & 52420.2 & 52412.4 & 7.8 & 52773.4 & 52779.7 & -6.3 & 176.6 \\
\hline 21 & 362 & 141803 & 2142 & 380 & 150 & 53343.8 & 53336.0 & 7.8 & 54076.4 & 54082.7 & -6.3 & 366.3 \\
\hline 21 & 362 & 141811 & 2144 & 380 & 145 & 53888.2 & 53880.4 & 7.8 & 54899.4 & 54905.6 & -6.2 & 505.6 \\
\hline 21 & 362 & 141819 & 2146 & 380 & 140 & 53684.6 & 53676.8 & 7.8 & 54660.4 & 1666.6 & -6.2 & 487.9 \\
\hline 21 & 362 & 141827 & 2148 & 380 & 135 & 52801.8 & 52793.9 & 7.9 & 52972.0 & 2978.2 & -6.2 & 85.1 \\
\hline 21 & 362 & 141836 & 2150 & 380 & 130 & 51459.4 & 51451.5 & .7 .9 & 51057.4 & 51063.6 & -6.2 & -201.0 \\
\hline 21 & 362 & 141844 & 2152 & 380 & 125 & 50766.2 & 50758.3 & 7.9 & 50340.8 & 50346.9 & -6.1 & -212.7 \\
\hline 21 & 362 & 141851 & 2154 & 380 & 120 & 50595.4 & 50587.5 & 7.9 & 50449.8 & 50455.9 & -6.1 & -72.8 \\
\hline 21 & 362 & 900 & 2156 & 380 & 115 & 50401.4 & 50393.4 & 8.0 & 50069.0 & 50075.1 & -6.1 & -166.2 \\
\hline 21 & 362 & 308 & 2158 & 380 & 110 & 50399.8 & 50391.8 & 8.0 & 49979.0 & 49985.1 & -6.1 & -210.4 \\
\hline 21 & 362 & 141915 & 2160 & 380 & 105 & 50782.4 & 50774.4 & 8.0 & 50506.8 & 50512.8 & -6.0 & -137.8 \\
\hline 21 & 362 & 141923 & 2162 & 380 & 100 & 51043.4 & 51035.4 & 8.0 & 50941.6 & 50947.6 & -6.0 & -50.9 \\
\hline 21 & 362 & 141930 & 2164 & 380 & 95 & 51099.0 & 51091.0 & 8.0 & 51000.2 & 51006.2 & -6.0 & -49.4 \\
\hline 21 & 362 & 141938 & 2166 & 380 & 90 & 51108.0 & 51099.9 & 8.1 & 51055.6 & 51061.6 & -6.0 & -26.2 \\
\hline 21 & 362 & 141945 & 2168 & 380 & 85 & 51128.4 & 51120.3 & 8.1 & 51098.6 & 51104.6 & -6.0 & -14.9 \\
\hline 21 & 362 & 141952 & 2170 & 380 & 80 & 51141.4 & 51133.3 & 8.1 & 51124.2 & 51130.1 & -5.9 & -8.6 \\
\hline 21 & 362 & 142000 & 2172 & 380 & 75 & 51137.8 & 51129.7 & 8.1 & 51130.8 & 51136.7 & -5.9 & -3.5 \\
\hline 21 & 362 & 142007 & 2174 & 380 & 70 & 51126.0 & 51117.9 & 8.1 & 51125.4 & 51131.3 & -5.9 & -0.3 \\
\hline 21 & 362 & 142015 & 2176 & 380 & 65 & 51088.2 & 51080.0 & 8.2 & 51110.2 & 51116.1 & -5.9 & 11.0 \\
\hline 21 & 362 & 142023 & 2178 & 380 & 60 & 51056.2 & 51048.0 & 8.2 & 51061.0 & 51066.8 & -5.8 & 2.4 \\
\hline 21 & 362 & 142032 & 2180 & 380 & 55 & 50983.2 & 50975.0 & 8.2 & 50993.4 & 50999.2 & -5.8 & 5.1 \\
\hline 21 & 362 & 142039 & 2182 & 380 & 50 & 50892.4 & 50884.2 & 8.2 & 50854.2 & 50860.0 & -5.8 & -19.1 \\
\hline 21 & 362 & 142047 & 2184 & 380 & 45 & 50772.4 & 50764.2 & 8.2 & 50521.4 & 50527.2 & -5.8 & -125.5 \\
\hline 21 & 362 & 142055 & 2186 & 380 & 40 & 50978.8 & 50970.5 & 8.3 & 50830.2 & 50836.0 & -5.8 & -74.3 \\
\hline 21 & 362 & 142103 & 2188 & 380 & 35 & 51561.0 & |51552.7 & 8.3 & 51683.4 & 51689.1 & -5.7 & 61.2 \\
\hline
\end{tabular}




\begin{tabular}{|c|c|c|c|c|c|c|c|c|c|c|c|c|}
\hline & & & & & & & กT) & & BOT & TOM (nT) & & Vert.Grad. \\
\hline Line & Day & Time & Sta. & $\mathbf{x}$ & $\mathbf{Y}$ & uncorr. & corr: & diff. & uncorr. & orr. & diff. & \\
\hline 21 & 362 & 142111 & 2190 & 380 & 30 & 52087.0 & 52078.7 & 8.3 & 52135.2 & 52140.9 & -5.7 & 24.1 \\
\hline 21 & 362 & 142119 & 2192 & 380 & 25 & 52636.2 & 52627.9 & 8.3 & 53081.4 & 53087.1 & -5.7 & 222.6 \\
\hline 21 & 362 & 142128 & 2194 & 380 & 20 & 52634.8 & 52626.4 & 8.4 & 53276.8 & 53282.5 & -5.7 & 321.0 \\
\hline 21 & 362 & 142136 & 2196 & $380 \mid$ & 15 & 52121.8 & 52113.4 & 8.4 & 52222.6 & 52228.2 & -5.6 & 50.4 \\
\hline 21 & 362 & 142143 & 2198 & 380 & 10 & 51707.2 & 51698.8 & 8.4 & 51655.6 & $|51661.2|$ & -5.6 & -25.8 \\
\hline 21 & 362 & 142152 & 2200 & $380 \mid$ & 5 & 1504.8 & 51496.4 & 8.4 & 51454.0 & 51459.6 & -5.6 & -25.4 \\
\hline 21 & 362 & 142200 & 2202 & $380 \mid$ & 0 & |431.4 & 51423.0 & 8.4 & 51393.6 & 51399.2 & -5.6 & -18.8 \\
\hline 22 & 362 & $|142950|$ & 2214 & $370 \mid$ & 0 & 51478.8 & 51469.9 & 8.9 & 51440.8 & $\mid 51445.6$ & -4.8 & -19.0 \\
\hline 22 & 362 & 142958 & 2216 & $370 \mid$ & 5 & 51605.0 & 51596.0 & 9.0 & 51543.6 & 51548.3 & -4.7 & -30.7 \\
\hline 22 & 362 & 143006 & 2218 & |370| & 10 & 1887.0 & 51878.0 & 9.0 & 51806.0 & 51810.7 & -4.7 & -40.5 \\
\hline 22 & 362 & 143013 & 2220 & $|370|$ & 15 & 52317.2 & 52308.2 & 9.0 & 52328.6 & 52333.3 & -4.7 & 5.7 \\
\hline 22 & $|362|$ & 143021 & 2222 & 370 & 20 & 52857.4 & 52848.4 & 9.0 & 53172.0 & 53176.7 & -4.7 & 157.3 \\
\hline 22 & $|362|$ & 143031 & 2224 & $370 \mid$ & 25 & 52945.4 & 52936.4 & 9.0 & 53296.4 & 301.0 & -4.6 & 175.5 \\
\hline 22 & 362 & 143149 & 2226 & $370 \mid$ & 30 & 52713.0 & 52703.9 & 9.1 & 53033.2 & 53037.7 & -4.5 & 160.1 \\
\hline 22 & 362 & 143157 & 2228 & $370 \mid$ & 35 & 375.8 & 52366.7 & 9.1 & 52569.0 & 52573.5 & -4.5 & 96.6. \\
\hline 22 & 362 & 143205 & 2230 & 370 & 40 & 52090.0 & 52080.9 & 9.1 & 52374.2 & 52378.7 & -4.5 & 142.1 \\
\hline 22 & 362 & 143214 & 2232 & 370 & 45 & 51579.4 & 51570.3 & 9.1 & 51621.0 & 51625.5 & -4.5 & 20.8 \\
\hline 22 & 362 & 143222 & 2234 & 370 & 50 & 51191.4 & 51182.3 & 9.1 & 51102.8 & 51107.3 & -4.5 & -44.3 \\
\hline 22 & $|362|$ & 143230 & 2236 & 370 & 55 & 51072.2 & 51063.1 & 9.1 & 51013.2 & 51017.6 & -4.4 & -29.5 \\
\hline 22 & 362 & 143238 & 2238 & $370 \mid$ & 60 & 51065.2 & 51056.1 & 9.1 & 51030.6 & 51035.0 & -4.4 & -17.3 \\
\hline 22 & 362 & 143248 & 2240 & 370 & $65^{\circ}$ & 51081.8 & 51072.6 & 9.2 & 51067.2 & 51071.6 & -4.4 & -7.3 \\
\hline 22 & 362 & 143255 & 2242 & 370 & 70 & 1.4 & 51092.2 & 9.2 & 51093.2 & 1097.6 & -4.4 & -4.1 \\
\hline 22 & 362 & 143303 & 2244 & 370 & 75 & 07.2 & 51098.0 & 9.2 & 51102.0 & 06.4 & -4.4 & -2.6 \\
\hline 22 & 362 & 143310 & 2246 & $370 \mid$ & 80 & 51110.6 & 51101.4 & 9:2 & 51085.6 & 51090.0 & -4.4 & -12.5 \\
\hline 22 & $362 \mid$ & 143318 & 2248 & $|370|$ & 85 & 51085.6 & 51076.4 & 9.2 & 51049.2 & 51053.6 & -4.4 & -18.2 \\
\hline 22 & 362 & 143326 & 2250 & $370 \mid$ & 90 & 51043.6 & 51034.4 & 9.2 & 50988.4 & 50992.7 & -4.3 & -27.6 \\
\hline 22 & 362 & 143333 & 2252 & 370 & 95 & 50988.0 & 50978.8 & 9.2 & 50905.0 & 50909.3 & -4.3 & -41.5 \\
\hline 22 & 362 & 143341 & 2254 & $370 \mid$ & 100 & 50928.0 & 50918.8 & 8.2 & 50790.4 & 50794.7 & -4.3 & -68.8 \\
\hline 22 & 362 & 143349 & 2256 & $|370|$ & 105 & 50893.6 & 50884.4 & 9.2 & 50750.8 & 50755.1 & -4.3 & -71.4 \\
\hline 22 & $362 \mid$ & 143357 & 2258 & 370 & 110 & 50953.4 & 50944.2 & 9.2 & 50879.0 & 50883.3 & -4.3 & -37.2 \\
\hline 22 & 362 & 143405 & 2260 & $370 \mid$ & 115 . & 50960.8 & 50951.6 & 9.2 & 50724.0 & 50728.3 & -4.3 & -118.4 \\
\hline 22 & 362 & 143414 & 2262 & $370 \mid$ & 120 & 50974.2 & 50965.0 & 9.2 & 50707.2 & 50711.5 & -4.3 & -133.5 \\
\hline 22 & 362 & 143422 & 2264 & $370 \mid$ & 125 & 51094.6 & 51085.4 & 9.2 & 50675.0 & 0679.3 & -4.3 & -209.8 \\
\hline 22 & 362 & 143430 & 2266 & 370 & 130 & 51602.8 & 51593.6 & 9.2 & 51249.2 & 51253.4 & -4.2 & -176.8 \\
\hline 22 & 362 & 143439 & 2268 & 370 & 135 & 52842.6 & 52833.3 & 9.3 & 53083.6 & 53087.8 & -4.2 & 120.5 \\
\hline 22 & $.362 \mid$ & 143447 & 2270 & 370 & 140 & 53981.6 & 53972.3 & 9.3 & 55154.0 & 55158.2 & -4.2 & 586.2 \\
\hline 22 & 362 & 143456 & 2272 & 370 & 145 & 53572.4 & 53563.1 & 9.3 & 54440.0 & 54444.2 & -4.2 & 433.8 \\
\hline 22 & 362 & 143504 & 2274 & 370 & 150 & 52653.2 & 52643.9 & 9.3 & 52809.4 & 52813.6 & -4.2 & 78.1 \\
\hline 22 & 362 & 143512 & 2276 & 370 & 155 & 51589.6 & 51580.3 & 9.3 & 51375.2 & 51379.4 & -4.2 & -107.2 \\
\hline 22 & 362 & 143520 & 2278 & 370 & 160 & 50801.6 & 50792.3 & 9.3 & 50113.4 & 50117.6 & -4.2 & -344.1 \\
\hline 22 & 362 & 143528 & 2280 & 370 & 165 & 50640.2 & 50630.9 & 9.3 & 50301.8 & 50305.9 & -4.1 & -169.2 \\
\hline 22 & 362 & 143538 & 2282 & 370 & 170 & 50396.8 & 50387.5 & 9.3 & 49970.6 & 49974.7 & -4.1 & -213.1 \\
\hline 22 & 362 & 143546 & 2284 & 370 & 175 & 50474.2 & 50464.9 & 9.3 & 50227.6 & 50231.7 & -4.1 & -123.3 \\
\hline 22 & 362 & 143554 & 2286 & 370 & 180 & 50693.0 & 50683.7 & 9.3 & 50485.6 & 50489.7 & -4.1 & $-103: 7$ \\
\hline 22 & 362 & 143602 & 2288 & 370 & 185 & 50910.2 & 50900.9 & 9.3 & 50577.6 & 50581.7 & -4.1 & -166.3 \\
\hline 22 & 362 & 143610 & 2290 & 370 & 190 & 51320.4 & 51311.1 & 9.3 & 51083.0 & 51087.1 & -4.1 & -118.7 \\
\hline 22 & 362 & 143618 & 2292 & 370 & 195 & 51976.2 & 51966.9 & 9.3 & 52291.2 & 52295.3 & -4.1 & 157.5 \\
\hline .22 & 362 & 143626 & 2294 & $370 \mid$ & 200 & 52373.2 & 52363.8 & 9.4 & 53303.2 & 53307.2 & -4.0 & 465.0 \\
\hline & 362 & 143634 & 2296 & $|370|$ & 205 & 51916.4 & 51907.0 & 9.4 & 52432.4 & 52436.4 & -4.0 & 258.0 \\
\hline
\end{tabular}




\begin{tabular}{|c|c|c|c|c|c|c|c|c|c|c|c|c|}
\hline & & & & & & & $\mathrm{SP}(\mathrm{nT})$ & & BOT & TOM (nT & & Vert.Grad. \\
\hline Line & Day & Time & Sta & $\mathbf{x}$ & $Y$ & incorr. & corr. & dif & uncorr. & corr & diff. & \\
\hline 22 & 362 & 143642 & 2298 & 370 & 210 & 51039.0 & 51029.6 & 9.4 & 50737.0 & 50741.0 & -4.0 & -151.0 \\
\hline 22 & 362 & 143650 & 2300 & 370 & 215 & 50822.0 & 50812.6 & 9.4 & 50371.6 & 50375.6 & -4.0 & -225.2 \\
\hline 22 & 362 & 143659 & 2302 & 370 & $220^{\circ}$ & 50822.0 & 50812.6 & 9.4 & 50664.2 & 50668.2 & -4.0 & -78.9 \\
\hline 22 & 362 & 143707 & 2304 & 370 & 225 & 50982.4 & 50973.0 & 9.4 & 50981.0 & 50985.0 & -4.0 & -0.7 \\
\hline 22 & 362 & 143714 & 2306 & 370 & 230 & 51107.8 & 51098.4 & 9.4 & 51119.4 & 51123.4 & -4.0 & 5.8 \\
\hline 23 & 362 & 144051 & 2308 & 360 & 230 & 51086.0 & 51076.4 & 9.6 & 51097.4 & 51101.0 & -3.6 & 5.7 \\
\hline 23 & 362 & 144059 & 2310 & 360 & 225 & 51031.0 & 51021.4 & 9.6 & 51020.8 & 51024.4 & -3.6 & -5.1 \\
\hline 23 & 362 & 144106 & 2312 & 360 & 220 & 50927.4 & 50917.8 & 9.6 & 50845.4 & 50849.0 & -3.6 & -41.0 \\
\hline 23 & 362 & 144115 & 2314 & 360 & 215 & 50856.6 & 50847.0 & $9.6^{-}$ & 50585.0 & 50588.6 & -3.6 & -135.8 \\
\hline 23 & 362 & 144123 & 2316 & 360 & 210 & 51174.4 & 51164.8 & 9.6 & 50846.2 & 50849.8 & -3.6 & -164.1 \\
\hline 23 & 362 & 144131 & 2318 & 360 & 205 & 1962.8 & 953.2 & 9.6 & 52075.0 & 52078.5 & -3.5 & 56.1 \\
\hline 23 & 362 & 144138 & 2320 & 360 & 200 & 52487.0 & 52477.4 & 9.6 & 52652.0 & 52655.5 & -3.5 & 82.5 \\
\hline 23 & 362 & 144146 & 2322 & 360 & 195 & 52765.6 & 52755.9 & 9.7 & 53080.6 & 53084.1 & -3.5 & 157.5 \\
\hline 23 & 362 & 144154 & 2324 & 360 & 190 & 52662.4 & 52652.7 & 9.7 & 53132.2 & 53135.7 & -3.5 & 234.9 \\
\hline 23 & 362 & 144202 & 2326 & 360 & 185 & 52015.8 & 52006.1 & 9.7 & 52192.0 & 52195.5 & -3.5 & 88.1 \\
\hline 23 & 362 & 144210 & 2328 & 360 & 180 & 51372.6 & 51362.8 & 9.7 & 51379.6 & 51383.1 & -3.5 & 3.5 \\
\hline 23 & 362 & 144219 & 2330 & 360 & 175 & 50818.8 & 50809.1 & 9.7 & 50716.8 & 50720.3 & -3.5 & -51.0 \\
\hline 23 & 362 & 144227 & 2332 & 360 & 170 & 50334.4 & 50324.7 & 9.7 & 49999.8 & 50003.2 & -3.4 & -167.3 \\
\hline 23 & 362 & 144235 & 2334 & 360 & 165 & 50353.2 & 50343.5 & 9.7 & 49925.4 & 49928.8 & -3.4 & -213.9 \\
\hline 23 & 362 & 144243 & 2336 & 360 & 160 & 50412.8 & 50403.1 & 9.7 & 50040.8 & 50044.2 & -3.4 & -186.0 \\
\hline 23 & 362 & 144251 & 2338 & 360 & 155 & 50942.2 & 50932.5 & 9.7 & 50939.4 & 50942.8 & -3.4 & -1.4 \\
\hline 23 & 362 & 144259 & 2340 & 360 & 150 & 51565.8 & 556.1 & 9.7 & 3.4 & 51366.8 & -3.4 & -101.2 \\
\hline 23 & 362 & 144307 & 2342 & 360 & 145 & 52227.8 & 52218.1 & 9.7 & 51927.6 & 51931.0 & -3.4 & -150.1 \\
\hline 23 & 362 & 144315 & 2344 & 360 & 140 & 52916.2 & 52906.5 & 9.7 & 53702.2 & 53705.6 & -3.4 & 393.0 \\
\hline 23 & 362 & 144322 & 2346 & 360 & 135 & 52494.8 & 52485.1 & 9.7 & 52880.8 & 52884.2 & -3.4 & 193.0 \\
\hline 23 & 362 & 144330 & 2348 & 360 & 130 & 51585.6 & 51575.8 & 9.8 & 51166.2 & 51169.5 & -3.3 & -209.7 . \\
\hline 23 & 362 & 144338 & 2350 & 360 & 125 & 51533.8 & 51524.0 & 9.8 & 51510.2 & 51513.5 & -3.3 & -11.8 \\
\hline .23 & 362 & 144345 & 2352 & 360 & 120 & 51913.2 & 51903.4 & 9.8 & 52676.8 & 52680.1 & -3.3 & 381.8 \\
\hline 23 & 362 & 144353 & 2354 & 360 & 115 & 51834.6 & 51824.8 & 9.8 & 52352.0 & 52355.3 & -3.3 & 258.7 \\
\hline 23 & 362 & 144401 & 2356 & 360 & 110 & 51516.6 & 51506.8 & 9.8 & 51620.8 & 51624.1 & -3.3 & 52.1 \\
\hline 23 & 362 & $\mid 144408$ & 2358 & 360 & 105 & 51392.0 & 382.2 & 9.8 & 2.4 & 51535.7 & -3.3 & 70.2 \\
\hline 23 & 362 & 144416 & 2360 & 360 & 100 & 51243.6 & 51233.8 & 9.8 & 32.0 & 51235.3 & -3.3 & -5.8 \\
\hline 23 & 362 & 144423 & 2362 & 360 & 95 & 51160.8 & 51151.0 & 9.8 & 51112.8 & 51116.1 & -3.3 & -24.0 \\
\hline 23 & 362 & 144430 & 2364 & 360 & 90 & 51126.4 & 51116.6 & 9.8 & 51091.0 & 51094.2 & -3.2 & -17.7 \\
\hline 23 & 362 & 144438 & 2366 & 360 & 85 & 51113.6 & 51103.8 & 9.8 & 51107.0 & 51110.2 & -3.2 & -3.3 \\
\hline 23 & 362 & 144446 & 2368 & 360 & 80 & 51113.8 & 51104.0 & 9.8 & 1115.2 & 51118.4 & -3.2 & 0.7 \\
\hline 23 & 362 & 144454 & 2370 & 360 & 75 & 51110.0 & 51100.2 & 9.8 & 5.1103 .2 & 51106.4 & -3.2 & -3.4 \\
\hline 23 & 362 & 144501 & 2372 & 360 & 70 & 51082.8 & 51073.0 & 9.8 & 51076.8 & 51080.0 & -3.2 & -3.0 \\
\hline 23 & 362 & 144510 & 2374 & 360 & 65 & 51028.2 & 51018.4 & 9.8 & 19.8 & 51023.0 & -3.2 & $-4.2^{\circ}$ \\
\hline 23 & 362 & 144517 & 2376 & 360 & 60 & 50963.6 & 953.7 & 9.9 & 27.4 & 50930.6 & -3.2 . & -18.1 \\
\hline 23 & 362 & 144525 & 2378 & 360 & 55 & 50902.2 & 50892.3 & 9.9 & 50802.6 & 50805.7 & -3.1 & -49.8 \\
\hline 23 & 362 & 144533 & 2380 & 360 & 50 & 50995.6 & 50985.7 & 9.9 & 50789.4 & 50792.5 & -3.1 & -103.1 \\
\hline 23 & 362 & 144541 & 2382 & 360 & 45 & 51527.2 & 51517.3 & 9.9 & 51608.8 & 51611.9 & -3.1 & 40.8 \\
\hline 23 & 362 & 144549 & 2384 & 360 & 40 & 52269.8 & 52259.9 & 9.9 & 53209.2 & 53212.3 & -3.1 & 469.7 \\
\hline 23 & 362 & 144557 & 2386 & 360 & 35 & 52420.2 & 52410.3 & 9.9 & 52858.4 & 52861.5 & -3.1 & 219.1 \\
\hline 23 & 362 & 144605 & 2388 & 360 & 30 & 52568.8 & 52558.9 & 9.9 & 52752.0 & 52755.1 & -3.1 & 91.6 \\
\hline 23 & 362 & 144614 & 2390 & 360 & 25 & 53232.2 & 53222.3 & 9.9 & 53761.8 & 53764.9 & -3.1 & 264.8 \\
\hline 23 & 362 & 144622 & 2392 & 360 & 20 & 53234.0 & 53224.1 & 9.9 & 53938.2 & 53941.3 & -3.1 & 352.1 \\
\hline 23 & 362 & 144630 & 2394 & 36 & 15 & 52556.8 & 52546.9 & 9.9 & 52634.0 & 52637.0 & -3.0 & 38.6 \\
\hline
\end{tabular}




\begin{tabular}{|c|c|c|c|c|c|c|c|c|c|c|c|c|}
\hline Line & Day & Time & Sta. & $\mathbf{X}$ & $\mathbf{Y}$ & uncorr. & $\begin{array}{l}P \text { (nT) } \\
\text { corr. }\end{array}$ & diff. & $\begin{array}{l}\text { BOT } \\
\text { uncorr. }\end{array}$ & $\begin{array}{l}\text { TOM (nT } \\
\text { corr. }\end{array}$ & diff. & $\begin{array}{c}\text { Vert.Grad. } \\
\text { (nT/ft) }\end{array}$ \\
\hline 23 & 362 & 144638 & 2396 & 360 & 10 & 51933.0 & 51923.1 & 9.9 & 51855.2 & 51858.2 & -3.0 & -38.9 \\
\hline 23 & 362 & 144647 & 2398 & 360 & 5 & 51610.6 & 51600.7 & 9.9 & 51547.6 & 51550.6 & -3.0 & -31.5 \\
\hline 23 & 362 & 144656 & 2400 & 360 & 0 & 51473.8 & 51463.9 & 9.9 & 51418.4 & 51421.4 & -3.0 & -27.7 \\
\hline 24 & 362 & 145228 & 2412 & 350 & 0 & 51402.8 & 51391.1 & 11.7 & 51338.0 & 51340.5 & -2.5 & -32.4 \\
\hline 24 & 362 & 145236 & 2414 & 350 & 5 & 51539.0 & 51527.2 & $11: 8$ & 51431.6 & 51434.0 & -2.4 & -53.7 \\
\hline 24 & 362 & 145244 & 2416 & 350 & 10 & 51865.6 & 51853.7 & 11.9 & 51704.8 & 51707.2 & -2.4 & -80.4 \\
\hline 24 & 362 & 145253 & 2418 & 350 & 15 & 52692.8 & 52680.9 & 11.9 & 52751.6 & 52754.0 & -2.4 & 29.4 \\
\hline 24 & 362 & 145301 & 2420 & 350 & 20 & 53974.4 & 53962.4 & 12.0 & 51988.0 & 51990.4 & -2.4 & -993.2 \\
\hline 24 & 362 & 145309 & 2422 & 350 & 25 & 53103.6 & 53091.6 & 12.0 & 54182.2 & 54184.6 & -2.4 & 539.3 \\
\hline 24 & 362 & 145317 & 2424 & 350 & 30 & 51526.6 & 514.5 & 12.1 & 50811.6 & 814.0 & -2.4 & -357.5 \\
\hline 24 & 362 & 145325 & 2426 & 350 & 35 & 50744.6 & 50732.5 & 12.1 & 50000.0 & 50002.3 & -2.3 & -372.3 \\
\hline 24 & 362 & 145333 & 2428 & 350 & 40 & 50576.8 & 50564.6 & 12.2 & 50275.8 & 50278.1 & -2.3 & -150.5 \\
\hline 24 & 362 & 145340 & 2430 & 350 & 45 & 50430.6 & 50418.4 & 12.2 & 50008.6 & 50010.9 & -2.3 & -211.0 \\
\hline 24 & 362 & 145348 & 2432 & 350 & 50 & 50504.2 & 50491.9 & 12.3 & 50232.4 & 50234.7 & -2.3 & -135.9 \\
\hline 24 & 362 & 45355 & 2434 & 350 & 55 & 50702.6 & 50690.3 & 12.3 & 50598.0 & 50600.3 & -2.3 & -52.3 \\
\hline 24 & 362 & 145402 & 2436 & 350 & 60 & 50883.6 & 50871.2 & 12.4 & 50848.6 & 50850.9 & -2.3 & -17.5 \\
\hline 24 & 362 & 145410 & 2438 & 350 & 65 & 51003.4 & 50991.0 & 12.4 & 50993.4 & 50995.7 & -2.3 & -5.0 \\
\hline 24 & 362 & 145418 & 2440 & 350 & 70 & 51084.4 & 51071.9 & 12.5 & 51076.0 & 51078.2 & -2.2 & -4.2 \\
\hline 24 & 362 & 145426 & 2442 & 350 & 75 & 51131.0 & 51118.5 & $.12 .5^{\prime}$ & 51125.0 & 51127.2 & -2.2 & -3.0 \\
\hline 24 & 362 & 145434 & 2444 & 350 & 80 & .51153 .2 & 51140.6 & 12.6 & 51145.2 & 51147.4 & -2.2 & -4.0 \\
\hline 24 & 362 & 145442 & 2446 & 350 & 85 & 51174.4 & 51161.8 & 12.6 & 51156.8 & 51159.0 & -2.2 & -8.8 \\
\hline 24 & 362 & 145451 & 2448 & 350 & 90 & 51218.0 & 51205.3 & 12.7 & 51175.2 & 51177.4 & -2.2 & -21.4 \\
\hline 24 & $|362|$ & 145459 & 2450 & 350 & 95 & 51347.6 & 51334.9 & 12.7 & 51303.8 & 51306.0 & -2.2 & -21.9 \\
\hline 24 & 362 & 145507 & 2452 & 350 & 100 & 51653.6 & 51640.8 & 12.8 & 51795.4 & 51797.6 & -2.2 & 70.9 \\
\hline 24 & 362 & 145515 & 2454 & 350 & 105 & 51829.6 & 51816.8 & 12.8 & 52437.6 & 52439.7 & -2.1 & 304.0 \\
\hline 24 & 362 & 145524 & 2456 & 350 & 110 & 51328.4 & 51315.5 & 12.9 & 51136.2 & 51138.3 & -2.1 & -96.1 \\
\hline 24 & 362 & 145532 & 2458 & 350 & 115 & 51001.8 & 50988.9 & 12.9 & 50599.6 & 50601.7 & -2.1 & -201.1 \\
\hline 24 & 362 & 145541 & 2460 & 350 & 120 & 50988.4 & 50975.4 & 13.0 & $50661: 6$ & 50663.7 & -2.1 & -163.4 \\
\hline 24 & 362 & 145549 & 2462 & 350 & 125 & 51025.4 & 51012.3 & 13.1 & 50725.0 & 50727.1 & -2.1 & -150.2 \\
\hline 24 & 362 & 145557 & 2464 & 350 & 130 & 51254.4 & 51241.3 & 13.1 & 50857.2 & 50859.3 & -2.1 & -198.6 \\
\hline 24 & 362 & 145605 & 2466 & 350 & 135 & 51980.2 & 51967.0 & 13.2 & 51825.4 & 51827.5 & -2.1 & -77.4 \\
\hline 24 & 362 & 145614 & 2468 & 350 & 140 & 52665.0 & 52651.8 & 13.2 & 53732.2 & 53734.2 & -2.0 & 533.6 \\
\hline 24 & 362 & 145622 & 2470 & 350 & 145 & 52518.6 & 52505.3 & 13.3 & 53268.8 & 53270.8 & -2.0 & 375.1 \\
\hline 24 & 362 & 145630 & 2472 & 350 & 150 & 51494.8 & 51481.5 & 13.3 & 51527.4 & 51529.4 & -2.0 & 16.3 \\
\hline 24 & 362 & 145639 & 2474 & 350 & 155 & 50566.2 & 50552.8 & 13.4 & 50123.0 & 50125.0 & -2.0 & -221.6 \\
\hline 24 & 362 & 145646 & 2476 & 350 & 160. & 50167.0 & 50153.6 & 13.4 & 49498.8 & 500.8 & -2.0 & -334.1 \\
\hline 24 & 362 & 145654 & 2478 & 350 & 165 & 50435.4 & 50421.9 & 13.5 & 50149.2 & 51.2 & -2.0 & -143.1 \\
\hline 24 & 362 & 145702 & 2480 & 350 & 170 & 50573.0 & 50559.5 & 13.5 & 50188.4 & 50190.4 & -2.0 & -192.3 \\
\hline 24 & 362 & 145710 & 2482 & 350 & 175 & 51094.6 & 51081.0 & 13.6 & 51018.4 & 51020.4 & -2.0 & -38.1 \\
\hline 24 & 362 & 145719 & 2484 & 350 & 180 & 51953.4 & 51939.8 & 13.6 & 52064.4 & 52066.3 & $-1.9^{\prime}$ & 55.5 \\
\hline 24 & 362 & 145727 & 2486 & 350 & 185 & 52846.2 & 52832.5 & 13.7 & 53332.8 & 53334.7 & -1.9 & 243.3 \\
\hline 24 & 362 & 145735 & 2488 & 350 & 190 & 53843.0 & 53829.3 & 13.7 & 55037.4 & 55039.3 & -1.9 & 597.2 \\
\hline 24 & 362 & 145743 & 2490 & 350 & 195 & 53995.2 & 53981.4 & 13.8 & 55346.6 & 55348.5 & -1.9 & 675.7 \\
\hline 24 & 362 & 145753 & 2492 & 350 & 200 & 52995.2 & 52981.3 & 13.9 & 53390.4 & 53392.3 & -1.9 & 197.6 \\
\hline 24 & 362 & 145801 & 2494 & 350 & 205 & 52053.8 & 52039.9 & 13.9 & 51893.6 & 51895.5 & -1.9 & -80.1 \\
\hline 24 & $362 \mid$ & 145810 & 2496 & 350 & 210 & 51368.6 & 51354.6 & 14.0 & 51112.4 & 114.2 & -1.8 & -128.1 \\
\hline 24 & 362 & 145818 & 2498 & 350 & 215 & 51032.2 & 51018.2 & 14.0 & 50824.8 & 50826.6 & -1.8 & -103.7 \\
\hline 24 & 362 & 145826 & 2500 & 350 & 220 & 51006.2 & 50992.1 & 14.1 & 50905.4 & 50907.2 & -1.8 & -50.4 \\
\hline 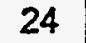 & $|362|$ & 145833 & 2502 & & 225 & 51063.6 & 51049.5 & 14.1 & 51029.0 & 51030.8 & -1.8 & -17.3 \\
\hline
\end{tabular}




\begin{tabular}{|c|c|c|c|c|c|c|c|c|c|c|c|c|}
\hline & & & & & & & $\mathrm{P} P(\mathrm{nT})$ & & $\mathrm{BO}$ & TOM (nT & & Vert.Grad. \\
\hline ine & Day & Time & Cts & $\mathrm{x}$ & $\mathbf{Y}$ & uncorr. & corr. & diff. & uncorr. & corr. & diff. & \\
\hline 24 & 362 & 145841 & 2504 & 350 & 230 & 51142.4 & 51128.2 & 14.2 & 51129.4 & 51131.2 & -1.8 & -6.5 \\
\hline 25 & 362 & 150206 & 2506 & 340 & 230 & 51091.2 & 51075.7 & 15.5 & 51072.2 & 51073.6 & -1.4 & -9.5 \\
\hline 25 & 362 & 150214 & 2508 & 340 & 225 & 51031.4 & 51015.9 & 15.5 & 50976.8 & 50978.2 & -1.4 & -27.3 \\
\hline 25 & 362 & 150222 & 2510 & 340 & 220 & 50966.4 & 50950.8 & 15.6 & 50838.6 & 50840.0 & -1.4 & -63.9 \\
\hline 25 & 362 & 150230 & 2512 & 340 & 215 & 51026.6 & 51011.0 & 15.6 & 50811.2 & 50812.6 & -1.4 & -107.7 \\
\hline 25 & 362 & 150238 & 2514 & 340 & 210 & 51340.4 & $|51324.7|$ & 15.7 & 51153.8 & 51155.2 & -1.4 & -93.3 \\
\hline 25 & 362 & $|150246|$ & 2516 & 340 & 205 & 51908.4 & |51892.7 & 15.7 & 51881.2 & 51882.6 & -1.4 & -13.6 \\
\hline 25 & 362 & 150253 & 2518 & 340 & 200 & 52475.2 & 52459.4 & 15.8 & 52431.8 & 52433.2 & -1.4 & -21.7 \\
\hline 25 & 362 & 150301 & 2520 & 340 & 195 & 52711.4 & 52695.6 & 15.8 & 52454.6 & 52456.0 & -1.4 & -128.4 \\
\hline 25 & 362 & 150309 & 2522 & 340 & 190 & 52647.2 & 52631.3 & 15.9 & 52667.6 & 52668.9 & -1.3 & 10.2 \\
\hline 25 & 362 & 150317 & 2524 & 340 & 185 & 52237.2 & 52221.3 & 15.9 & 52255.0 & 52256.3 & $=1.3$ & 8.9 \\
\hline 25 & 362 & 150325 & 2526 & 340 & 180 & 51661.0 & 51645.0 & 16.0 & 51637.4 & 51638.7 & -1.3 & -11.8 \\
\hline 25 & 362 & 150333 & 2528 & 340 & 175 & 51056.6 & 51040.6 & 16.0 & 50930.4 & 0931.7 & -1.3 & -63.1 \\
\hline 25 & 362 & 150341 & 2530 & 340 & 170 & 50553.8 & 50537.7 & 16.1 & 50110.8 & 50112.1 & -1.3 & -221.5 \\
\hline 25 & 362 & 150350 & 2532 & 340 & 165 & 50108.6 & 50092.4 & 16.2 & 49701.2 & 49702.5 & -1.3 & -203.7 \\
\hline 25 & 362 & 150359 & 2534 & 340 & 160 & 49719.6 & 49703.4 & 16.2 & 49219.0 & 49220.3 & -1.3 & -250.3 \\
\hline 25 & 362 & 150407 & 2536 & 340 & 155 & 49900.4 & 49884.1 & 16.3 & 49405.2 & 49406.4 & -1.2 & -247.6 \\
\hline 25 & 362 & 150414 & 2538 & 340 & 150 & 50777.8 & $|50761.5|$ & 16.3 & 50893.2 & 0894.4 & -1.2 & 57.7 \\
\hline 25 & 362 & 150422 & 2540 & 340 & 145 & 51694.4 & 51678.0 & 16.4 & 52426.2 & 52427.4 & -1.2 & 365.9 \\
\hline 25 & 362 & 150430 & 2542 & 340 & 140 & 52165.2 & 52148.8 & 16.4 & 52961.8 & 52963.0 & -1.2 & 3998.3 \\
\hline 25 & 362 & 150438 & 2544 & 340 & 135 & 51704.8 & 51688.3 & 16.5 & 51850.2 & 51851.4 & -1.2 & 72.7 \\
\hline 25 & 362 & 150446 & 2546 & 340 & 130 & 51053.6 & 51037.1 & 16.5 & 50756.4 & 0757.6 & -1.2 & -148.6 \\
\hline 25 & 362 & 150453 & 2548 & 340 & 125 & 51022.6 & $|51006.0|$ & 16.6 & 50905.6 & 50906.8 & -1.2 & -58.5 \\
\hline 25 & 362 & $|150502|$ & 2550 & 340 & 120 & 51123.8 & 51107.2 & 16.6 & 51256.8 & 51257.9 & -1.1 & 66.5 \\
\hline 25 & 362 & 150510 & 2552 & 340 & 115 & 51006.0 & 50989.3 & 16.7 & 50816.8 & 50817.9 & -1.1 & -94.6 \\
\hline 25 & 362 & 150519 & 2554 & 340 & 110 & 51052.0 & 51035.3 & 16.7 & 50717.8 & 50718.9 & -1.1 & -167.1 \\
\hline 25 & 362 & 150526 & 2556 & 340 & 105 & 51371.8 & $|51355.0|$ & 16.8 & 51217.4 & 51218.5 & -1.1 & -77.2 \\
\hline 25 & 362 & 150534 & 2558 & 340 & $\cdot 100$ & 51409.6 & 51392.8 & 16.8 & 51355.8 & 51356.9 & -1.1 & -26.9 \\
\hline 25 & 362 & 150542 & 2560 & 340 & 95 & 51294.8 & $51277.9 \mid$ & 16.9 & 51218.2 & 51219.3 & -1.1 & -38.3 \\
\hline 25 & 362 & 150550 & 2562 & 340 & 90 & 51244.0 & 51227.1 & 16.9 & 51209.0 & 51210.1 & -1.1 & -17.5 \\
\hline 25 & 362 & 150557 & 2564 & 340 & 85 & 51225.6 & $\mid 51208.6$ & 17.0 & 51205.2 & 51206.2 & -1.0 & -10.2 \\
\hline 25 & 362 & 150605 & 2566 & 340 & 80 & 51202.6 & 51185.6 & 17.0 & 51196.6 & 51197.6 & -1.0 & -3.0 \\
\hline 25 & 362 & 150613 & 2568 & 340 & 75 & 51169.6 & 51152.5 & 17.1 & 51173.8 & 51174.8 & -1.0 & 2.1 \\
\hline 25 & 362 & 150621 & 2570 & 340 & 70 & 51115.2 & $\mid 51098.1$ & 17.1 & 51119.8 & 51120.8 & -1.0 & 2.3 \\
\hline 25 & 362 & 150629 & 2572 & 340 & 65 & 51024.4 & 51007.2 & 17.2 & 51028.4 & 51029.4 & -1.0 & 2.0 \\
\hline 25 & 362 & 150635 & 2574 & 340 & 60 & 50886.8 & $|50869.6|$ & 17.2 & 50872.4 & 50873.4 & -1.0 & -7.2 \\
\hline 25 & 362 & 150643 & 2576 & 340 & 55 & 50664.2 & 50646.9 & 17.3 & 50610.2 & 50611.2 & -1.0 & -27.0 \\
\hline 25 & 362 & 150651 & 2578 & 340 & 50 & 50404.2 & 50386.9 & 17.3 & 50225.6 & 50226.6 & -1.0 & -89.3 \\
\hline 25 & 362 & 150659 & 2580 & 340 & 45 & 50171.0 & 50153.6 & 17.4 & 49840.2 & 49841.1 & -0.9 & -165.4 \\
\hline 25 & 362 & 150708 & 2582 & 340 & 40 & 50007.0 & |49989.6 & 17.4 & 49445.6 & 49446.5 & -0.9 & -280.7 \\
\hline 25 & 362 & 150716 & 2584 & 340 & 35 & 50336.4 & 50318.9 & 17.5 & 49538.2 & 49539.1 & -0.9 & -399.1 \\
\hline 25 & 362 & 150723 & 2586 & 340 & 30 & 51778.2 & 51760.7 & 17.5 & 51847.2 & 51848.1 & -0.9 & 34.5 \\
\hline 25 & 362 & 150731 & 2588 & 340 & 25 & 53705.6 & 53688.0 & 17.6 & 54835.0 & 54835.9 & -0.9 & 564.7 \\
\hline 25 & 362 & 150743 & 2590 & 340 & 20 & 53370.0 & 53352.3 & 17.7 & 54106.0 & 54106.9 & -0.9 & 368.0 \\
\hline 25 & 362 & 150752 & 2592 & 340 & 15 & 52181.8 & 52164.1 & 17.7 & 52048.6 & 52049.5 & -0.9 & -66.6 \\
\hline 25 & 362 & 150801 & 2594 & 340 & 10 & 51632.6 & 51614.8 & 17.8 & 51438.6 & 51439.4 & -0.8 & -97.0 \\
\hline 25 & 362 & 150810 & 2596 & 340 & 5 & 51377.2 & 51359.4 & 17.8 & 51283.2 & 51284.0 & -0.8 & -47.0 \\
\hline 25 & 362 & 150818 & 2598 & 340 & 0 & 51314.6 & 51296.7 & 17.9 & 51252.2 & 51253.0 & -0.8 & -31.2 \\
\hline 26 & 17 & 95357 & 10 & 330 & 0 & 51221.2 & 51211.0 & 10.2 & 51165.4 & 51179.0 & -13 & -27.9 \\
\hline
\end{tabular}




\begin{tabular}{|c|c|c|c|c|c|c|c|c|c|c|c|c|}
\hline Line & Day & Time & Sta. & $\mathbf{x}$ & $\mathbf{Y}$ & uncorr. & $\begin{array}{l}(n T) \\
\text { corr. }\end{array}$ & diff. & $\begin{array}{l}\text { BOT } \\
\text { uncorr. }\end{array}$ & $\begin{array}{c}\text { TOM (nT) } \\
\text { corr. }\end{array}$ & diff. & $\begin{array}{c}\text { Vert.Grad. } \\
\text { (nT/ft) }\end{array}$ \\
\hline 26 & 17 & 95406 & $\overline{12}$ & 330 & 5 & 51209.8 & 51199.6 & 10.2 & 51113.4 & 51127.0 & -13.6 & -48.2 \\
\hline 26 & 17 & 95426 & 14 & $330 \mid$ & 10 & 51259.2 & 51249.1 & 10.1 & 51116.6 & 51130.3 & $|-13.7|$ & -71.3 \\
\hline 26 & 17 & 95435 & 16 & $|330|$ & 15 & 439.6 & 51429.5 & 10.1 & 51320.4 & 51334.2 & $\mid-13.8$ & $-59.6^{\circ}$ \\
\hline 26 & 17 & 95448 & 18 & $|330|$ & 20 & 51844.2 & 51834.1 & 10.1 & 52155.8 & 52169.6 & $\mid-13.8$ & 155.8 \\
\hline 26 & 17 & 95457 & 20 & $330 \mid$ & 25 & 51618.0 & 51608.0 & 10.0 & 51814.4 & 51828.3 & $|-13.9|$ & 98.2 \\
\hline 26 & 17 & 95505 & 22 & $330 \mid$ & 30 & 50627.6 & 50617.6 & 10.0 & 50282.2 & 50296.1 & $\mid-13.9$ & -172.7 \\
\hline 26 & 17 & 95512 & 24 & $330 \mid$ & 35 & 50033.0 & 50023.0 & 10.0 & 49602.2 & 49616.2 & $|-14.0|$ & -215.4 \\
\hline 26 & 17 & 95520 & 26 & $330 \mid$ & 40 & 49973.6 & 49963.6 & 10.0 & 49508.8 & 49522.8 & $\mid-14.0$ & -232.4 \\
\hline 26 & 17 & 95528 & 28 & $|330|$ & 45 & 50143.6 & 50133.7 & 9.9 & 49803.4 & 49817.5 & $|-14.1|$ & -170.1 \\
\hline 26 & 17 & 95535 & 30 & $330 \mid$ & 50 & 50452.0 & 50442.1 & 9.9 & 50323.0 & 50337.1 & $|-14.1|$ & -64.5 \\
\hline 26. & 17 & 95542 & 32 & 330 & 55 & 50746.4 & 50736.5 & 9.9 & 50716.4 & 50730.6 & $\mid-14.2$ & -15.0 \\
\hline 26 & 17 & 95550 & 34 & $330 \mid$ & 60 & 50946.4 & 50936.5 & 9.9 & 50949.4 & 50963.6 & $|-14.2|$ & 1.5 \\
\hline 26 & 17 & 95558 & 36 & 330 & 65 & 51079.2 & 51069.3 & 9.9 & 51086.4 & 51100.6 & -14.2 & 3.6 \\
\hline 26 & 17 & 95605 & 38 & $330 \mid$ & 70 & 51161.6 & 51151.8 & 9.8 & 5.1165 .2 & 51179.5 & $\mid-14.3$ & 1.8 \\
\hline 26 & 17 & 95613 & 40 & 330 & 75 & 51214.8 & 51205.0 & 9.8 & 51212.2 & 51226.5 & $\mid-14.3$ & -1.3 \\
\hline 26 & 17 & 95620 & 42 & $330 \mid$ & 80 & 51247.4 & 51237.6 & 9.8 & 51239.4 & 51253.8 & $\mid-14.4$ & -4.0 \\
\hline 26 & 17 & 95628 & 44 & $330 \mid$ & 85 & 51279.6 & 51269.8 & 9.8 & 51258.2 & 51272.6 & $|-14.4|$ & -10.7 \\
\hline 26 & 17 & 95636 & 46 & $|330|$ & 90 & 51318.6 & 51308.9 & 9.7 & 51283.8 & 51298.3 & $\mid-14.5$ & -17.4 \\
\hline 26 & 17 & 95643 & 48 & 330 & 95 & 51355.4 & 51345.7 & 9.7 & 51294.4 & 51308.9 & $\mid-14.5$ & -30.5 \\
\hline 26 & 17 & 95650 & 50 & $|330|$ & 100 & 51413.4 & 51403.7 & 9.7 & 51312.6 & 51327.1 & $|-14.5|$ & -50.4 \\
\hline 26 & 17 & 95658 & 52 & $|330|$ & 105 & 51445.4 & 51435.7 & 9.7 & 51346.8 & 51361.4 & $\mid-14.6$ & -49.3 \\
\hline 26 & 17 & 95706 & 54 & $330 \mid$ & .110 & 51398.6 & 51389.0 & 9.6 & 51393.4 & 51408.0 & -14.6 & -2.6 \\
\hline 26 & 17 & 95714 & 56 & 330 & 115 & 51168.6 & 51159.0 & 9.6 & 51019.6 & 51034.3 & $|-14.7|$ & -74.5 \\
\hline 26 & 17 & 95722 & 58 & 330 & 120 & 50919.2 & 50909.6 & 9.6 & 50559.8 & 50574.5 & -14.7 & -179.7 \\
\hline 26 & 17 & 95730 & 60 & $|330|$ & 125 & 50899.6 & 50890.0 & 9.6 & 50883.4 & 50898.2 & $\mid-14.8$ & -8.1 \\
\hline 26 & 17 & 95738 & 62 & 330 & 130 & 50804.2 & 50794.7 & 9.5 & 50521.4 & 50536.2 & -14.8 & -141.4 \\
\hline 26 & 17 & 95745 & 64 & 330 & 135 & 50873.4 & 50863.9 & 9.5 & 50866.8 & 50881.7 & $\mid-14.9$ & -3.3 \\
\hline 26 & 17 & 95753 & 66 & 330 & 140 & 50590.6 & 50581.1 & 9.5 & 50754.8 & 50769.7 & $\mid-14.9$ & 82.1 \\
\hline 26 & 17 & 95801 & 68 & $330 \mid$ & 145 & 49795.2 & 49785.7 & 9.5 & 48961.2 & 48976.1 & $|-14.9|$ & -417.0 \\
\hline 26 & 17 & 95809 & 70 & $|330|$ & 150 & 49107.2 & 49097.7 & 9.5 & 48235.4 & 48250.4 & $\mid-15.0$ & -435.9 \\
\hline 26 & 17 & 95817 & 72 & 330 & 155 & 48516.2 & 48506.8 & 9.4 & 58560.2 & 58575.2 & -15.0 & 5022.0 \\
\hline 26 & 17 & 95825 & 74 & 330 & 160 & 48915.6 & 48906.2 & 9.4 & 47754.6 & 47769.7 & $\mid-15.1$ & -580.5 \\
\hline 26 & 17 & 95833 & 76 & $330 \mid$ & 165 & 49889.4 & 49880.0 & 9.4 & 48853.0 & 48868.1 & -15.1 & -518.2 \\
\hline 26 & 17 & 95842 & 78 & $330 \mid$ & 170 & 50705.8 & 50696.4 & 9.4 & 50349.4 & 50364.6 & $|-15.2|$ & -178.2 \\
\hline 26 & 17 & 95849 & 80 & $330 \mid$ & 175 & $51270: 6$ & 51261.3 & 9.3 & 51176.6 & 51191.8 & $|-15.2|$ & -47.0 \\
\hline 26 & 17 & 95856 & 82 & 330 & 180 & 51815.6 & 51806.3 & 9.3 & 51778.4 & 51793.7 & -15.3 & -18.6 \\
\hline 26 & 17 & 95904 & 84 & $330 \mid$ & 185 & 0.2 & 52440.9 & 9.3 & 52510.8 & 52526.1 & $|-15.3|$ & 30.3 \\
\hline 26 & 17 & 95912 & 86 & $330 \mid$ & 190 & 53264.8 & 53255.5 & 9.3 & 53723.4 & 53738.7 & $\mid-15.3$ & 229.3 \\
\hline 26 & 17 & 95924 & 88 & 330 & 195 & 53957.0 & 53947.8 & 9.2 & 58583.2 & 58598.6 & $\mid-15.4$ & 2313.1 \\
\hline 26 & 17 & 95931 & 90 & 330 & 200 & 53201.6 & 53192.4 & 9.2 & 53795.8 & 53811.3 & $\mid-15.5$ & 297.1 \\
\hline 26 & 17 & 95940 & 92 & $330 \mid$ & 205 & 51948.4 & 51939.2 & 9.2 & 51746.4 & 51761.9 & $|-15.5|$ & -101.0 \\
\hline 26 & 17 & 95947 & 94 & $330 \mid$ & 210 & 51099.2 & 51090.0 & 9.2 & 50784.8 & 50800.3 & $\mid-15.5$ & -157.2 \\
\hline 26 & 17 & 95955 & 96 & $330 \mid$ & 215 & 50798.4 & 50789.3 & 9.1 & 50490.2 & 50505.8 & $\mid-15.6$ & -154.1 \\
\hline 26 & 17 & 100002 & 98 & $330 \mid$ & 220 & 50825.0 & 50815.9 & 9.1 & 50663.2 & 50678.8 & $\mid-15.6$ & -80.9 \\
\hline 26 & 17 & 100010 & 100 & 330 & 225 & 50947.4 & 50938.3 & 9.1 & 50875.2 & 50890.9 & $|-15.7|$ & -36.1 \\
\hline 26 & 17 & 100017 & 102 & $330 \mid$ & 230 & 51065.8 & 51056.7 & 9.1 & 51032.6 & 51048.3 & $|-15.7|$ & -16.6 \\
\hline 26 & 17 & 100024 & 104 & 330 & 235 & 51153.6 & 51144.6 & 9.0 & 51141.4 & 51157.1 & $|-15.7|$ & -6.1 \\
\hline 26 & 17 & 100031 & 106 & 330 & 240 & 51219.6 & 51210.6 & 9.0 & 51213.6 & 51229.4 & -15.8 & -3.0 \\
\hline 26 & 17 & 100038 & 108 & 330 & 245 & 51259.8 & 51250.8 & 9.0 & .51261 .2 & 51277.0 & $|-15.8|$ & 0.7 \\
\hline
\end{tabular}




\begin{tabular}{|c|c|c|c|c|c|c|c|c|c|c|c|c|}
\hline & & & & & & & $P(n T)$ & & BOT & TOM (nT) & dit & $\begin{array}{c}\text { Vert.Grad. } \\
\text { (nTfft) }\end{array}$ \\
\hline Ine & Iuv & Time & & $X$ & $\boldsymbol{Y}$ & & & & & & aim. & \\
\hline 26 & 17 & 100141 & 110 & 330 & 250 & 51308.6 & 51299.8 & 8.8 & 51312.8 & 51329.0 & -16.2 & 2.1 \\
\hline 26 & 17 & 100150 & 112 & 330 & 255 & 51336.2 & 51327.4 & 8.8 & 51341.2 & 51357.4 & -16.2 & 2.5 \\
\hline 26 & 17 & 100200 & 114 & 330 & 260 & 51353.6 & 51344.8 & 8.8 & 51360.0 & 51376.3 & $|-16.3|$ & 3.2 \\
\hline 26 & 17 & 100209 & $116^{\circ}$ & 330 & 265 & 51372.6 & 51363.9 & 8.7 & 51378.2 & 51394.5 & -16.3 & 2.8 \\
\hline 26 & 17 & 100218 & 118 & 330 & 270 & 51385.0 & 51376.3 & 8.7 & 51388.2 & 51404.6 & -16.4 & 1.6 \\
\hline 26 & 17 & 100226 & 120 & 330 & 275 & 51397.0 & 51388.3 & 8.7 & 51400.8 & 51417.2 & -16.4 & 1.9 \\
\hline 26 & 17 & 100234 & 122 & 330 & 280 & 1405.0 & 51396.3 & 8.7 & 51401.8 & 51418.3 & -16.5 & -1.6 \\
\hline 26 & 17 & 100247 & 124 & 330 & 285 & 51414.4 & 51405.8 & 8.6 & 51416.8 & 51433.4 & -16.6 & 1.2 \\
\hline 27 & 17 & 100726 & 126 & 320 & 295 & 1425.6 & 51417.8 & 7.8 & 51427.4 & 51445.5 & -18.1 & 0.9 \\
\hline 27 & 17 & 100736 & 128 & 320 & 290 & 51421.4 & 51413.7 & 7.7 & 51422.0 & 51440.2 & -18.2 & 0.3 \\
\hline 27 & 17 & 100745 & 130 & 320 & 285 & 51416.6 & 51408.9 & 7.7 & 51419.4 & 51437.6 & -18.2 & 1.4 \\
\hline 27 & 17 & 100755 & 132 & 320 & 280 & 32.6 & 51394.9 & 7.7 & 51409.6 & 51427.9 & -18.3 & 3.5 \\
\hline 27 & 17 & 100803 & 134 & 320 & 275 & 90.4 & 51382.7 & 7.7 & 51391.4 & 409.7 & -18.3 & 0.5 \\
\hline 27 & 17 & 100811 & 136 & 320 & 270 & 51381.6 & 51374.0 & 7.6 & 51380.4 & 51398.8 & $|-18.4|$ & -0.6 \\
\hline 27 & 17 & 100828 & 138 & 320 & 265 & 371.0 & 51363.4 & 7.6 & 51375.2 & 51393.7 & $|-18.5|$ & 2.1 \\
\hline 27 & 17 & 100836 & 140 & 320 & 260 & 52.6 & 51345.0 & 7.6 & 51361.4 & 51379.9 & $|-18.5|$ & 4.4 \\
\hline 27 & 17 & 100843 & 142 & 320 & 255 & 30.8 & 51323.3 & 7.5 & 51341.2 & 51359.8 & $|-18.6|$ & 5.2 \\
\hline 27 & 17 & 100851 & 144 & 320 & 250 & 05.2 & |297.7 & 7.5 & 51312.6 & 331.2 & $|-18.6|$ & 3.7 \\
\hline 27 & 17 & 100859 & 146 & 320 & 245 & 268.2 & 51260.7 & 7.5 & 51273.4 & 1292.1 & $|-18.7|$ & 2.6 \\
\hline 27 & 17 & 100906 & 148 & 320 & 240 & 1216.6 & 51209.1 & 7.5 & 51220.2 & 1238.9 & -18.7 & 1.8 \\
\hline 27 & 17 & 100914 & 150 & 320 & 235 & 157.4 & 149.9 & 7.5 & 51150.8 & 169.5 & $|-18.7|$ & -3.3 \\
\hline 27 & 17 & 100923 & 152 & 320 & 230 & 1057.2 & 049.8 & 7.4 & 51032:0 & 050.8 & -18.8 & -12.6 \\
\hline 27 & 17 & 100930 & 154 & 320 & 225 & 9937.0 & 50929.6 & 7.4 & 50896.0 & 914.8 & -18.8 & -20.5 \\
\hline 27 & 17 & 100938 & 156 & 320 & 220 & 0779.8 & |50772.4 & 7.4 & 50668.4 & 687.3 & $|-18.9|$ & -55.7 \\
\hline 27 & 17 & 100945 & 158 & 320 & 215 & 01.0 & 50593.6 & 7.4 & 50342.0 & 50360.9 & $|-18.9|$ & -129.5 \\
\hline 27 & 17 & 100953 & 160 & 320 & 210 & $\$ 8.0$ & 50540.7 & 7.3 & 50014.0 & 50033.0 & $\mid-19.0$ & -267.0 \\
\hline 27 & 17 & 101000 & 162 & 320 & 205 & 14.6 & 51087.3 & 7.3 & 50431.8 & 50450.8 & $\mid-19.0$ & -331.4 \\
\hline 27 & 17 & 101007 & 164 & 320 & 200 & 52716.6 & 52709.3 & 7.3 & 52994.4 & 013.5 & $|-19.1|$ & 138.9 \\
\hline 27 & 17 & 101014 & 166 & 320 & 195 & 54751.2 & 54743.9 & 7.3 & 52059.2 & 52078.3 & $|-19.1|$ & -1346.0 \\
\hline 27 & 17 & 101022 & 168 & 320 & 190 & 52256.6 & 52249.4 & 7.2 & 52154.2 & 52173.3 & -19.1 & -51.2 \\
\hline 27 & 17 & 101029 & 170 & 320 & 185 & 53144.2 & 137.0 & 7.2 & 53585.0 & 53604.2 & -19.2 & 220.4 \\
\hline 27 & 17 & 101037 & 172 & 320 & 180 & 52221.6 & 52214.4 & 7.2 & 52274.2 & 2293.4 & -19.2 & 26.3 \\
\hline 27 & 17 & 101045 & 174 & 320 & 175 & 51697.0 & 51689.8 & 7.2 & 51665.2 & 1684.5 & -19.3 & -15.9 \\
\hline 27 & 17 & 110 & 176 & 320 & 170 & 51453.0 & 445.8 & 7.2 & 51351.8 & 371.1 & $|-19.3|$ & -50.6 \\
\hline 27 & 17 & 10 & 178 & 320 & 165 & $51283: 8$ & $|51276.7|$ & 7.1 & 51261.8 & |281.1 & $|-19.3|$ & -11.0 \\
\hline 27 & 17 & 101 & 180 & 320 & 160 & 50957.8 & $|50950.7|$ & 7.1 & 51071.8 & 1091.2 & $|-19.4|$ & 57.0 \\
\hline 27 & 17 & 101116 & 182 & 320 & 155 & 50621.8 & 50614.7 & 7.1 & 50586.0 & 50605.4 & -19.4 & -17.9 \\
\hline 27 & 17 & 10 & 184 & 320 & 150 & 50550.4 & 50543.3 & 7.1 & 50250.6 & 50270.1 & $\mid-19.5$ & -149.9 \\
\hline 27 & 17 & 101133 & 186 & 320 & 145 & 50588.4 & 581.4 & 7.0 & 50288.6 & 0308.1 & -19.5 & -149.9 \\
\hline 27 & 17 & 10 & 188 & 320 & 140 & 50776.8 & 769.8 & 7.0 & 78.6 & 50598.2 & $\mid-19.6$ & -99.1 \\
\hline 27 & 17 & 101148 & 190 & 320 & 135 & 50975.8 & 50968.8 & 7.0 & 50841.6 & 0861:2 & -19.6 & -67.1 \\
\hline 27 & 17 & 101156 & 192 & 320 & 130 & 51066.2 & 51059.2 & 7.0 & 50967.6 & 50987.3 & -19.7 & -49.3 \\
\hline 27 & 17 & 101203 & 194 & 320 & 125 & $51133: 4$ & 51126.5 & 6.9 & 51045.6 & 51065.3 & $|-19.7|$ & -43.9 \\
\hline 27 & 17 & 101210 & 196 & 320 & 120 & 51261.6 & 51254.7 & 6.9 & 51151.8 & 51171.5 & $|-19.7|$ & -54.9 \\
\hline 27 & 17 & 101218 & 198 & 320 & 115 & 51629.0 & 51622.1 & 6.9 & 51602.2 & 51622.0 & $|-19.8|$ & -13.4 \\
\hline 27 & 17 & 101226 & 200 & 320 & 110 & 52306.2 & |52299.3 & 6.9 & 52804.6 & 52824.4 & $|-19.8|$ & 249.2 \\
\hline 27 & 17 & 101233 & 202 & 320 & 105 & 52591.2 & 52584.3 & 6.9 & 53741.8 & 53761.7 & $\mid-19.9$ & 575.3 \\
\hline 27 & 17 & 101240 & 204 & 320 & 100 & 52304.4 & 52297.6 & 6.8 & 52682.0 & 52701.9 & $\mid-19.9$ & 188.8 \\
\hline רי & 17 & 101248 & 206 & 320 & 95 & 51761.4 & $\mid 51754.6$ & 6.8 & 51804.8 & |51824.8 & $\mid-20.0$ & 21.7 \\
\hline
\end{tabular}




\begin{tabular}{|c|c|c|c|c|c|c|c|c|c|c|c|c|}
\hline Line & Day & Time & Sta. & $\mathbf{x}$ & $\mathbf{Y}$ & uncorr. & $\begin{array}{l}\text { SP (nT) } \\
\text { corr. }\end{array}$ & diff. & $\begin{array}{l}\mathrm{BO} \\
\text { uncorr. }\end{array}$ & $\begin{array}{l}\text { TOM (nT } \\
\text { corr. }\end{array}$ & | diff. & $\begin{array}{l}\text { Vert.Grad. } \\
\text { (nT/ft) }\end{array}$ \\
\hline 27 & 17 & 101256 & 208 & 320 & 90 & 51512.0 & 51505.2 & 6.8 & 51498.2 & 51518.2 & -20.0 & -6.9 \\
\hline 27 & 17 & 101304 & 210 & 320 & 85. & 51377.8 & 51371.0 & .6 .8 & 51366.4 & 51386.4 & -20.0 & -5.7 \\
\hline 27 & 17 & 101311 & 212 & 320 & 80 & 51319.0 & 51312.3 & 6.7 & 51313.2 & 51333.3 & -20.1 & -2.9 \\
\hline 27 & 17 & 101318 & 214 & $|320|$ & 75 & 51281.0 & 51274.3 & 6.7 & 51281.0 & 51301.1 & $|-20.1|$ & 0.0 \\
\hline 27 & 17 & 101325 & 216 & 320 & 70 & 51236.4 & 51229.7 & 6.7 & 51253.0 & 51273.2 & $\mid-20.2$ & 8.3 \\
\hline 27 & 17 & 101333 & 218 & 320 & 65 & 51183.8 & 51177.1 & 6.7 & 51201.0 & 51221.2 & $|-20.2|$ & 8.6 \\
\hline 27 & 17 & 101340 & 220 & $320 \mid$ & 60 & 51092.2 & 51085.6 & 6.6 & 51114.0 & 51134.3 & $|-20.3|$ & 10.9 \\
\hline 27 & 17 & 101348 & 222 & 320 & 55 & 50956.8 & $50950.2^{\circ}$ & 6.6 & 50969.8 & 50990.1 & $\mid-20.3$ & 6.5 \\
\hline 27 & 17 & 101355 & 224 & 320 & 50 & 50744.0 & 50737.4 & 6.6 & 50708.8 & 50729.1 & $\mid-20.3$ & -17.6 \\
\hline 27 & 17 & 101403 & 226 & $|320|$ & 45 & 50521.2 & 50514.6 & 6.6 & 50337.2 & 50357.6 & $|-20.4|$ & -92.0 \\
\hline 27 & 17 & 101438 & 228 & 320 & .40 & 50319.4 & 50312.9 & 6.5 & 50116.6 & 50137.2 & $\mid-20.6$ & -101.4 \\
\hline 27 & 17 & 101446 & 230 & 320 & 35 & 50213.0 & 50206.5 & 6.5 & 49953.0 & 49973.6 & -20.6 & -130.0 \\
\hline 27 & 17 & 101454 & 232 & 320 & 30 & 50254.6 & 50248.2 & 6.4 & 49775.0 & 49795.7 & $\mid-20.7$ & -239.8 \\
\hline 27 & 17 & 101501 & 234 & 320 & 25 & 50834.6 & 50828.2 & 6.4 & 50686.2 & 50706.9 & -20.7 & -74.2 \\
\hline 27 & 17 & 101509 & 236 & 320 & 20 & 51461.2 & 51454.8 & 6.4 & 51993.6 & 52014.4 & $\mid-20.8$ & 266.2 \\
\hline 27 & 17 & 101517 & 238 & 320 & 15 & 51337.4 & 51331.0 & 6.4 & 51345.0 & 51365.8 & $\mid-20.8$ & 3.8 \\
\hline 27 & 17 & 101525 & 240 & 320 & 10 & 51185.8 & 51179.5 & 6.3 & 51088.8 & 51109.6 & $\mid-20.8$ & -48.5 \\
\hline 27 & 17 & 101532 & 242 & 320 & 5 & 51176.6 & 51170.3 & 6.3 & 51111.2 & 51132.1 & -20.9 & -32.7 \\
\hline 27 & 17 & 101539 & 244 & 320 & 0 & 51205.0 & 51198.7 & 6.3 & 51162.8 & 51183.7 & -20.9 & -21.1 \\
\hline 28 & 17 & 101952 & 256 & 310 & 0 & 51226.4 & 51220.4 & 6.0 & 51194.4 & 51215.5 & -21.1 & -16.0 \\
\hline 28 & 17 & 102001 & 258 & 310 & 5 & 51177.4 & 51171.5 & 5.9 & 51115.8 & 51137.0 & -21.2 & -30.8 \\
\hline 28 & 17 & 102008 & 260 & 310 & 10 & 51165.2 & 51159.3 & 5.9 & 51049.6 & 51070.8 & $\mid-21.2$ & -57.8 \\
\hline 28 & 17 & 102015 & 262 & 310 & 15 & 51228.4 & 51222.5 & 5.9 & 51072.0 & 51093.2 & $\mid-21.2$ & -78.2 \\
\hline 28 & 17 & 102024 & 264 & 310 & 20 & 51304.0 & 51298.1 & 5.9 & 51397.6 & 51418.8 & $\mid-21.2$ & 46.8 \\
\hline 28 & 17 & 102032 & 266 & 310 & 25 & 50867.8 & 50861.9 & 5.9 & 50677.0 & 50698.2 & -21.2 & -95.4 \\
\hline 28 & 17 & 102040 & 268 & 310 & 30 & 50492.2 & 50486.3 & 5.9 & 50026.6 & 50047.8 & -21.2 & -232.8 \\
\hline 28 & 17 & 102051 & 270 & 310 & 35 & 50504.6 & 50498.7 & 5.9 & 50070.8 & 50092.0 & -21.2 & -216.9 \\
\hline 28 & 17 & 102057 & 272 & 310 & 40 & 50727.4 & $50721: 5$ & 5.9 & 50723.0 & 50744.2 & -21.2 & -2.2 \\
\hline 28 & 17 & 102106 & 274 & 310 & 45 & 50743.0 & 50737.1 & 5.9 & 50485.6 & 50506.8 & -21.2 & -128.7 \\
\hline 28 & 17 & 102113 & 276 & 310 & 50 & 50857.0 & 50851.1 & .5 .9 & 50768.4 & 50789.6 & -21.2 & -44.3 \\
\hline 28 & 17 & 102121 & 278 & 310 & 55 & 51025.2 & 51019.3 & 5.9 & 51019.4 & 51040.6 & -21.2 & -2.9 \\
\hline 28 & 17 & 102129 & 280 & 310 & 60 & 51158.0 & 51152.2 & 5.8 & 51166.6 & 51187.8 & -21.2 & 4.3 \\
\hline 28 & 17 & 102137 & 282 & 310 & 65 & 51231.2 & 51225.4 & $5.8^{\circ}$ & 51237.4 & 51258.7 & $\mid-21.3$ & 3.1 . \\
\hline 28 & 17 & 102144 & 284 & 310 & 70 & 51273.6 & 51267.8 & 5.8 & 51277.0 & 51298.3 & -21.3 & 1.7 \\
\hline 28 & 17 & 102152 & 286 & 310 & 75 & 51308.2 & 51302.4 & 5.8 & 51298.0 & 51319.3 & $\mid-21.3$ & -5.1 \\
\hline 28 & 17 & 102159 & 288 & 310 & 80 & 51328.0 & 51322.2 & 5.8 & $51313.0^{\circ}$ & 51334.3 & $\mid-21.3$ & -7.5 \\
\hline 28 & 17 & 102208 & 290 & 310 & 85 & 51368.4 & 51362.6 & 5.8 & 51329.2 & 51350.5 & -21.3 & -19.6 \\
\hline 28 & 17 & 102215 & 292 & 310 & 90 & 51447.2 & 51441.4 & 5.8 & 51385.0 & 51406.3 & -21.3 & -31.1 \\
\hline 28 & 17 & 102222 & 294 & 310 & 95 & 51632.4 & 51626.6 & 5.8 & 51562.0 & 51583.3 & $|-21.3|$ & -35.2 \\
\hline 28 & 17 & $|102230|$ & 296 & 310 & 100 & 51983.0 & 51977.2 & 5.8 & 52051.2 & 52072.5 & -21.3 & 34.1 \\
\hline 28 & 17 & 102239 & 298 & 310 & 105 & 52451.6 & 52445.8 & 5.8 & 53126.2 & 53147.5 & $\mid-21.3$ & 337.3 \\
\hline 28 & 17 & 102247 & 300 & 310 & 110 & 52234.0 & 52228.2 & 5.8 & 52901.6 & 52922.9 & $\mid-21.3$ & 333.8 \\
\hline 28 & 17 & 102255 & 302 & 310 & 115 & 51501.6 & 51495.9 & 5.7 & 51252.6 & 51273.9 & -21.3 & -124.5 \\
\hline 28 & 17 & 102303 & 304 & 310 & 120 & 51306.0 & 51300.3 & 5.7 & 50945.2 & 50966.5 & -21.3 & -180.4 \\
\hline 28 & 17 & 102311 & 306 & 310 & 125 & 51475.2 & 51469.5 & 5.7 & 51490.2 & 51511.5 & -21.3 & 7.5 \\
\hline 28 & 17 & 102318 & 308 & 310 & 130 & 51605.6 & 51599.9 & 5.7 & 51511.2 & 51532.5 & -21.3 & -47.2 \\
\hline 28 & 17 & 102326 & 310 & 310 & 135 & 51913.2 & 51907.5 & 5.7 & 51867.0 & 51888.3 & -21.3 & -23.1 \\
\hline 28 & 17 & 102334 & 312 & 310 & 140 & 52342.6 & 52336.9 & 5.7 & 52530.2 & 52551.5 & $\mid-21.3$ & 93.8 \\
\hline 28 & 17 & 102341 & 314 & 310 & 145 & 52686.0 & 52680.3 & 5.7 & 53060.0 & 53081.3 & -21.3 & 187.0 \\
\hline
\end{tabular}




\begin{tabular}{|c|c|c|c|c|c|c|c|c|c|c|c|c|}
\hline \multirow[b]{2}{*}{ Line } & \multirow[b]{2}{*}{ Day } & \multirow[b]{2}{*}{ Time } & \multirow[b]{2}{*}{ Sta. } & \multirow[b]{2}{*}{$\mathbf{X}$} & \multirow[b]{2}{*}{$\mathbf{Y}$} & \multicolumn{3}{|c|}{ TOP (nT) } & \multicolumn{2}{|c|}{ BOTTOM (nT) } & \multirow[b]{2}{*}{ diff. } & \multirow{2}{*}{$\begin{array}{c}\text { Vert.Grad. } \\
\text { (nT/fit) }\end{array}$} \\
\hline & & & & & & uncorr. & corr. & diff. & uncorr. & corr. & & \\
\hline 28 & 17 & 102349 & 316 & 310 & 150 & 52894.4 & 52888.7 & 5.7 & 53623.4 & 53644.7 & $\mid-21.3$ & 364.5 \\
\hline 28 & 17 & 102358 & 318 & 310 & 155 & 52929.4 & 52923.7 & 5.7 & 53414.2 & 53435.5 & -21.3 & 242.4 \\
\hline 28 & 17 & 102407 & 320 & 310 & 160 & 52774.6 & 52768.9 & 5.7 & 58541.4 & 58562.7 & -21.3 & 2883.4 \\
\hline 28 & 17 & 102415 & 322 & 310 & 165 & 52253.8 & 52248.1 & 5.7 & 52403.0 & 52424.3 & $|-21.3|$ & 74.6 \\
\hline 28 & 17 & 102423 & 324 & 310 & 170 & 51645.8 & 51640.1 & 5.7 & 51404.0 & 51425.3 & $|-21.3|$ & -120.9 \\
\hline 28 & 17 & 102431 & 326 & 310 & 175 & 51553.2 & 51547.6 & 5.6 & 51401.0 & 51422.3 & $|-21.3|$ & -76.1 \\
\hline 28 & 17 & 102438 & 328 & 310 & 180 & 51807.2 & 51801.6 & 5.6 & 51688.0 & 51709.3 & $|-21.3|$ & -59.6 \\
\hline 28 & 17 & 102445 & 330 & 310 & 185 & 52236.0 & 52230.4 & 5.6 & 52297.6 & 52318.9 & $|-21.3|$ & 30.8 \\
\hline 28 & 17 & 102453 & 332 & 310 & 190 & 52770.2 & 52764.6 & 5.6 & 53107.2 & 53128.5 & $|-21.3|$ & 168.5 \\
\hline 28 & 17 & 102501 & 334 & 310 & 195 & 52563.0 & 52557.4 & 5.6 & 52988.2 & 53009.5 & -21.3 & 212.6 \\
\hline 28 & 17 & 102510 & 336 & 310 & 200 & 51274.0 & 51268.4 & 5.6 & 52080.6 & 52102.0 & -21.4 & 403.3 \\
\hline 28 & 17 & 102518 & 338 & 310 & 205 & 50627.8 & 50622.2 & 5.6 & 50096.0 & 50117.4 & $\mid-21.4$ & -265.9 \\
\hline 28 & 17 & 102526 & 340 & 310 & 210 & 50518.8 & 50513.2 & 5.6 & 50198.4 & 50219.8 & $|-21.4|$ & -160.2 \\
\hline 28 & 17 & 102534 & 342 & 310 & 215 & 50583.4 & 50577.8 & 5.6 & 50322.8 & 50344.2 & $-21.4 \mid$ & -130.3 \\
\hline 28 & 17 & 102542 & 344 & 310 & 220 & 50744.2 & 50738.6 & 5.6 & 50626.4 & 50647.8 & $|-21.4|$ & -58.9 \\
\hline 28 & 17 & 102549 & 346 & 310 & 225 & 50910.8 & 50905.2 & 5.6 & 50857.0 & 50878.4 & $|-21.4|$ & -26.9 \\
\hline 28 & 17 & 102556 & 348 & 310 & 230 & 51044.6 & 51039.1 & 5.5 & 51021.6 & 51043.0 & $|-21.4|$ & -11.5 \\
\hline 28 & 17 & 102604 & 350 & 310 & 235 & 51135.6 & 51130.1 & 5.5 & 51125.2 & 51146.6 & $\mid-21.4$ & -5.2 \\
\hline 28 & 17 & 102611 & 352 & 310 & 240 & 51213.2 & 51207.7 & 5.5 & 51200.4 & 51221.8 & $|-21.4|$ & -6.4 \\
\hline 28 & 17 & 102618 & 354 & 310 & 245 & 51260.6 & 51255.1 & 5.5 & 51251.2 & 51272.6 & $|-21.4|$ & -4.7 \\
\hline 28 & 17 & 102626 & 356 & 310 & 250 & 51294.4 & 51288.9 & 5.5 & 51291.6 & 51313.0 & $|-21.4|$ & -1.4 \\
\hline 28 & 17 & 102823 & 358 & 310 & 255 & 51327.6 & 322.2 & 5.4 & 324.6 & 46.0 & $|-21.4|$ & -1.5 \\
\hline 28 & 17 & 102832 & 360 & 310 & 260 & 51350.2 & 51344.8 & 5.4 & 51345.6 & 51367.0 & $|-21.4|$ & -2.3 \\
\hline 28 & 17 & 102840 & 362 & 310 & 265 & 51363.0 & 51357.6 & 5.4 & 51365.2 & 51386.7 & $|-21.5|$ & 1.1 \\
\hline 28 & 17 & 102853 & 364 & 310 & 270 & 51376.0 & 51370.6 & 5.4 & 51375.8 & 51397.3 & -21.5 & -0.1 \\
\hline 28 & 17 & 102902 & 366 & 310 & 275 & 51390.0 & 51384.7 & 5.3 & 51393.2 & 51414.7 & -21.5 & 1.6 \\
\hline 28 & 17 & 102911 & 368 & 310 & 280 & 51400.6 & 51395.3 & 5.3 & 51405.2 & 1426.7 & $|-21.5|$ & 2.3 \\
\hline 28 & 17 & 102919 & 370 & 310 & 285 & 51408.6 & 51403.3 & 5.3 & 51408.8 & 1430.3 & -21.5 & 0.1 \\
\hline 28 & 17 & 102928 & 372 & 310 & 290 & 51409.8 & 51404.5 & 5.3 & 51408.6 & 1430.1 & $\mid-21.5$ & -0.6 \\
\hline 28 & 17 & 102942 & 374 & 310 & 295 & 51420.6 & 51415.3 & 5.3 & 51420.8 & 51442.3 & $\mid-21.5$ & 0.1 \\
\hline 28 & 17 & 102954 & 376 & 310 & 300 & 51425.0 & 51419.7 & .5 .3 & 51426.2 & 51447.7 & -21.5 & 0.6 \\
\hline 29 & 17 & 103457 & 378 & 300 & 300 & 51422.4 & 51417.5 & 4.9 & 51418.6 & 51440.2 & $|-21.6|$ & -1.9 \\
\hline 29 & 17 & 103506 & 380 & 300 & 295 & 51420.8 & 51415.9 & 4.9 & 51422.6 & 1444.2 & $\mid-21.6$ & 0.9 \\
\hline 29 & 17 & 103517 & 382 & 300 & 290 & 51413.2 & 51408.3 & 4.9 & 51412.4 & 1434.0 & -21.6 & -0.4 \\
\hline 29 & 17 & 103530 & 384 & 300 & 285 & 51409.6 & 51404.7 & 4.9 & 51404.2 & 51425.8 & -21.6 & -2.7 \\
\hline 29 & 17 & 103539 & 386 & 300 & 280 & 51401.2 & 51396.3 & 4.9 & 51398.0 & 1419.6 & $|-21.6|$ & -1.6 \\
\hline 29 & 17 & 103604 & 388 & 300 & 275 & 51387.6 & 51382.7 & 4.9 & 51391.4 & 51413.1 & $|-21.7|$ & 1.9 \\
\hline 29 & 17 & 103613 & 390 & 300 & 270 & 51380.4 & 51375.5 & 4.9 & 51374.6 & 51396.3 & -21.7 & -2.9 \\
\hline 29 & 17 & 103621 & 392 & 300 & 265 & 51366.2 & 51361.3 & 4.9 & 51360.2 & -51381.9 & $|-21.7|$ & -3.0 \\
\hline 29 & 17 & 103634 & 394 & 300 & 260 & 51349.6 & 51344.8 & 4.8 & 51341.8 & 51363.5 & $-21.7 \mid$ & -3.9 \\
\hline 29 & 17 & 103646 & 396 & 300 & 255 & 51322.4 & 51317.6 & 4.8 & 51330.0 & 51351.7 & -21.7 & 3.8 \\
\hline 29 & 17 & 103654 & 398 & 300 & 250 & 51289.4 & 51284.6 & 4.8 & 51292.8 & 1314.5 & $\mid-21.7$ & 1.7 \\
\hline 29 & 17 & 103706 & 400 & 300 & 245 & 51245.4 & 51240.6 & 4.8 & 51252.8 & 51274.5 & $|-21.7|$ & 3.7 \\
\hline 29 & 17 & 103713 & 402 & 300 & 240 & 51198.8 & 51194.0 & 4.8 & 51192.0 & 51213.7 & $|-21.7|$ & -3.4 \\
\hline 29 & 17 & 103721 & 404 & 300 & 235 & 51114.0 & 51109.2 & 4.8 & 51111.2 & 51132.9 & -21.7 & -1.4 \\
\hline 29 & 17 & 103729 & 406 & 300 & 230 & 51017.8 & 51013.0 & 4.8 & 50991.2 & 51012.9 & $-21: 7$ & -13.3 \\
\hline 29 & 17 & 103736 & 408 & 300 & 225 & 50888.2 & 50883.4 & 4.8 & 50848.4 & 50870.1 & -21.7 & -19.9 \\
\hline 29 & 17 & 103744 & 410 & 300 & 220 & 50739.2 & 50734.4 & 4.8 & 50624.0 & 50645.7 & -21.7 & -57.6 \\
\hline 29 & 17 & 103752 & 412 & 300 & 215 & 50612.0 & 50607.2 & 4.8 & 50372.4 & | 50394.1 & $|-21.7|$ & -119.8 \\
\hline
\end{tabular}




\begin{tabular}{|c|c|c|c|c|c|c|c|c|c|c|c|c|}
\hline & & & & & & & $(n T)$ & & BO & TOM (nT & & Vert.Grad. \\
\hline Line & | Day & Time & Sta. & $\mathbf{x}$ & $\mathbf{Y}$ & uncorr. & corr. & | diff. & uncorr. & corr. & diff. & i) \\
\hline 29 & 17 & 103800 & 414 & 300 & 210 & 50597.6 & 50592.9 & 4.7 & 50178.8 & 50200.5 & -21.7 & -209.4 \\
\hline 29 & 17. & 103808 & 416 & 300 & 205 & 50775.6 & 50770.9 & 4.7 & 50275.4 & 50297.1 & -21.7 & -250.1 \\
\hline 29 & 17 & 103817 & 418 & 300 & 200 & 51258.6 & 51253.9 & 4.7 & 50613.4 & 50635.1 & $|-21.7|$ & -322.6 \\
\hline 29 & 17 & 103825 & 420 & 300 & 195 & 51970.4 & 51965.7 & 4.7 & 51951.6 & 51973.3 & $|-21.7|$ & -9.4 \\
\hline 29 & 17 & 103832 & 422 & 300 & 190 & 52075.4 & 52070.7 & 4.7 & 52149.8 & 52171.5 & $|-21.7|$ & 37.2 \\
\hline 29 & 17 & 103839 & 424 & 300 & 185 & 51757.0 & 51752.3 & 4.7 & 51677.8 & 51699.5 & $\mid-21.7$ & -39.6 \\
\hline 29 & 17 & 103846 & 426 & 300 & 180 & 51443.8 & 51439.1 & 4.7 & 51294.6 & 51316.3 & $|-21.7|$ & -74.6 \\
\hline 29 & 17 & 103854 & 428 & 300 & 175 & 51230.6 & 51225.9 & 4.7 & 50983.8 & 51005.5 & -21.7 & -123.4 \\
\hline 29 & 17 & 103902 & 430 & 300 & 170 & 51306.0 & 51301.3 & 4.7 & 50845.8 & 50867.5 & -21.7 & -230.1 \\
\hline 29 & 17 & 103910 & 432 & 300 & 165 & 51973.2 & 51968.5 & 4.7 & 51818.8 & 51840.5 & -21.7 & -77.2 \\
\hline 29 & 17 & 103919 & 434 & 300 & 160 & 53079.0 & 53074.3 & 4.7 & 54263.4 & 54285.1 & -21.7 & 592.2 \\
\hline 29 & 17 & 103927 & 436 & 300 & 155 & 53395.8 & 53391.1 & 4.7 & 54411.8 & 54433.6 & -21.8 & 508.0 \\
\hline 29 & 17 & 103935 & 438 & 300 & 150 & 53452.8 & 53448.2 & 4.6 & 54405.4 & 54427.2 & -21.8 & 476.3 \\
\hline 29 & 17 & 103943 & 440 & 300 & 145 & 53513.8 & 53509.2 & 4.6 & 54063.6 & 54085.4 & -21.8 & 274.9 \\
\hline 29 & 17 & 103951 & 442 & 300 & 140 & 53262.2 & 53257.6 & 4.6 & 53993.4 & 54015.2 & $|-21.8|$ & 365.6 \\
\hline 29 & 17 & 103958 & 444 & 300 & 135 & 52578.8 & 52574.2 & 4.6 & 52736.6 & 52758.4 & -21.8 & 78.9 \\
\hline 29 & 17 & 104006 & 446 & 300 & 130 & 51918.2 & 51913.6 & 4.6 & 51687.8 & 51709.6 & -21.8 & -115.2 \\
\hline 29 & 17 & 104014 & 448 & 300 & 125 & 51677.8 & 51673.2 & 4.6 & 51598.2 & 51620.0 & $|-21.8|$ & -39.8 \\
\hline 29 & 17 & 104022 & 450 & 300 & 120 & 51402.4 & 51397.8 & 4.6 & 51930.8 & 51952.6 & $|-21.8|$ & 264.2 \\
\hline 29 & 17 & 104030 & 452 & 300 & 115 & 50643.6 & 50639.0 & 4.6 & 49660.6 & 49682.4 & -21.8 & -491.5 \\
\hline 29 & 17 & 104037 & 454 & 300 & 110 & 50997.6 & 50993.0 & 4.6 & 50605.6 & 50627.4 & -21.8 & -196.0 \\
\hline 29 & 17 & 104046 & 456 & 300 & 105 & 51628.0 & 51623.4 & 4.6 & 52189.8 & 52211.6 & $|-21.8|$ & 280.9 \\
\hline 29 & 17 & 104054 & 458 & 300 & 100 & 31137.8 & 31133.2 & 4.6 & 52057.6 & 52079.4 & -21.8 & 10459.9 \\
\hline 29 & 17 & 104102 & 460 & 300 & 95 & 51454.2 & 51449.7 & 4.5 & 50964.2 & 50986.0 & -21.8 & -245.0 \\
\hline 29 & 17 & 104109 & 462 & 300 & 90 & 51309.4 & 51304.9 & 4.5 & 51219.2 & 51241.0 & -21.8 & -45.1 \\
\hline 29 & 17 & 104117 & 464 & 300 & 85 & 51327.8 & 51323.3 & 4.5 & 51230.6 & 51252.4 & -21.8 & -48.6 \\
\hline 29 & 17 & 104124 & 466 & 300 & 80 & 51408.6 & 51404.1 & $4.5^{\circ}$ & 51260.8 & 51282.6 & -21.8 & -73.9 \\
\hline 29 & 17 & 104131 & 468 & 300 & 75 & 51321.6 & 51317.1 & 4.5 & 51315.8 & 51337.6 & -21.8 & -2.9 \\
\hline 29 & 17 & 104138 & 470 & 300 & 70 & 51298.0 & 51293.5 & 4.5 & 51305.2 & 51327.0 & -21.8 & 3.6 \\
\hline 29 & 17 & 104145 & 472 & 300 & 65 & .51259 .2 & 51254.7 & 4.5 & 51276.6 & 51298.4 & -21.8 & 8.7 \\
\hline 29 & 17 & 104152 & 474 & 300 & 60. & 51175.0 & 51170.5 & 4.5 & 51192.4 & 51214.2 & -21.8 & 8.7 \\
\hline 29 & 17 & 104158 & 476 & 300 & 55 & 50980.4 & 50975.9 & 4.5 & 50957.8 & 50979.6 & -21.8 & -11.3 \\
\hline 29 & 17 & 104206 & 478 & 300 & 50 & 50720.2 & 50715.7 & 4.5 & 49801.2 & 49823.0 & -21.8 & -459.5 \\
\hline 29 & 17. & 104258 & 480 & 300 & 45 & 52266.0 & 52261.6 & 4.4 & 50456.4 & 50478.3 & $\mid-21.9$ & -904.8 \\
\hline 29 & 17 & 104306 & 482 & 300 & 40 & 51481.6 & 51477.2 & 4.4 & 52329.8 & 52351.7 & $|-21.9|$ & 424.1 \\
\hline 29 & 17 & 104314 & 484 & 300 & 35 & 50959.2 & 50954.8 & 4.4 & 50852.4 & 50874.3 & $|-21.9|$ & -53.4 \\
\hline 29 & 17 & 104322 & 486 & 300 & 30 & $50726: 6$ & 50722.2 & 4.4 & 50510.0 & 50531.9 & $\mid-21.9$ & -108.3 \\
\hline 29 & 17 & 104330 & 488 & 300 & 25 & 50286.4 & 50282.0 & 4.4 & 49103.2 & 49125.1 & -21.9 & -591.6 \\
\hline 29 & 17 & 104337 & 490 & 300 & 20 & 10698.6 & 10694.2 & 4.4 & 10507:6 & 10529.5 & $|-21.9|$ & -95.5 \\
\hline 29 & 17 & 104345 & 492 & 300 & 15 & 51154.4 & 51150.0 & 4.4 & 50346.4 & 50368.3 & -21.9 & -404.0 \\
\hline 29 & 17 & 104352 & 494 . & 300 & 10 & 51123.2 & 51118.8 & 4.4 & 50973.0 & 50994.9 & $\mid-21.9$ & -75.1 \\
\hline 29 & 17 & 104358 & 496 & 300 & 5 & 51197.6 & 51193.3 & 4.3 & 51155.2 & 51177.1 & -21.9 & -21.2 \\
\hline 29 & 17 & 104405 & 498 & 300 & 0 & 51244.8 & 51240.5 & 4.3 & 51224.6 & 51246.5 & -21.9 & -10.1 \\
\hline 30 & 17 & 115739 & 520 & 290 & 0 & 51250.2 & 51275.8 & -25.6 & 51237.6 & 51289.1 & -51.5 & -6.3 \\
\hline 30 & 17 & 115748 & 522 & 290 & 5 & 51195.2 & 51220.9 & -25.7 & 51173.2 & 51224.8 & -51.6 & -11.0 \\
\hline 30 & 17 & 115802 & 524 & 290 & 10 & 51094.4 & 51120.1 & $\mid-25.7$ & 51042.0 & 51093.6 & -5.1 .6 & -26.2 \\
\hline 30 & 17 & 115811 & 526 & 290 & 15 & 50928.0 & 50953.8 & -25.8 & 50804.2 & 50855.9 & -51.7 & -61.9 \\
\hline 30 & 17 & 115819 & 528 & 290 & 20 & 50697.0 & 50722.8 & -25.8 & 50460.0 & 50511.7 & $-51.7 \mid$ & -118.5 \\
\hline 30 & 17 & 115827 & 530 & 290 & 25 & 50520.6 & 50546.4 & -25.8 & 50077.0 & 50128.7 & -51.7 & -221.8 \\
\hline
\end{tabular}




\begin{tabular}{|c|c|c|c|c|c|c|c|c|c|c|c|c|}
\hline \multirow{2}{*}{ Line } & \multirow[b]{2}{*}{ Day } & \multirow[b]{2}{*}{ Time } & \multirow[b]{2}{*}{ Sta. } & \multirow[b]{2}{*}{$\mathbf{X}$} & \multirow[b]{2}{*}{$\mathbf{Y}$} & \multicolumn{2}{|c|}{ TOP (nT) } & \multirow[b]{2}{*}{ diff. } & \multicolumn{2}{|c|}{ BOTTOM (nT) } & \multirow[b]{2}{*}{ diff. } & \multirow{2}{*}{$\begin{array}{c}\text { Vert.Grad. } \\
\text { (nT/ft) }\end{array}$} \\
\hline & & & & & & uncorr. & corr. & & uncorr. & & & \\
\hline 30 & 17 & 115835 & 532 & 290 & 30 & 50436.8 & 50462.6 & -25.8 & 49735.8 & 49787.5 & -51.7 & -350.5 \\
\hline 30 & 17 & 15843 & 534 & 290 & 35 & 50596.2 & 50622.1 & -25.9 & 50358.0 & 50409.8 & -51.8 & -119.1 \\
\hline 30 & 17 & 115851 & 536 & 290 & 40 & 50340.4 & 50366.3 & $\mid-25.9$ & 49550.6 & 49602.4 & -51.8 & -394.9 \\
\hline 30 & 17 & 8,58 & 538 & 290 & 45 & 50375.2 & 50401.1 & -25.9 & 50073.0 & 50124.8 & -51.8 & -151.1 \\
\hline 30 & 17 & 115906 & 540 & 290 & 50 & 50797.4 & 50823.4 & -26.0 & 50783.8 & 50835.7 & -51.9 & -6.8 \\
\hline 30 & 17 & 115914 & 542 & 290 & 55 & 15.4 & 51141.4 & $\mid-26.0$ & 51128.4 & 51180.3 & $|-51.9|$ & 6.5 \\
\hline 30 & 17 & 115922 & 544 & 290 & 60 & 239.6 & 51265.6 & -26.0 & 51261.0 & 51312.9 & $|-51.9|$ & 10.7 \\
\hline 30 & 17 & 115929 & 546 & 290 & 65 & 51286.6 & 51312.6 & -26.0 & 51296.0 & 1347.9 & $|-51.9|$ & 4.7 \\
\hline 30 & 17 & 115936 & 548 & 290 & 70 & 51297.2 & 51323.3 & -26.1 & 51303.6 & 55.6 & $-52.0 \mid$ & 3.2 \\
\hline 30 & 17 & 115943 & 550 & 290 & 75 & 51294.0 & 51320.1 & -26.1 & 51281.6 & 51333.6 & $-52.0 \mid$ & -6.2 \\
\hline 30 & 17 & 950 & 552 & 290 & 80 & 70.0 & 51296.1 & -26.1 & 51230.4 & 1282.4 & $|-52.0|$ & -19.8 \\
\hline 30 & 17 & 958 & 554 & 290 & 85 & 47.0 & 51273.1 & -26.1 & 51213.6 & 1265.6 & $|-52.0|$ & -16.7 \\
\hline 30 & 17 & 120005 & 556 & 290 & 90 & 51200.4 & 51226.6 & -26.2 & 51128.6 & 1180.7 & $|-52.1|$ & -35.9 \\
\hline 30 & 17 & 120013 & 558 & 290 & 95 & 51100.2 & 51126.4 & -26.2 & 50967.2 & 10.19 .3 & $|-52.1|$ & -66.5 \\
\hline 30 & 17 & 120020 & 560 & 290 & 100 & 97.2 & 51023.4 & $|-26.2|$ & 50826.2 & 08878.3 & $|-52.1|$ & -85.5 \\
\hline 30 & 17 & 120029 & 562 & 290 & 105 & 39.0 & 50965.3 & $-26.3 \mid$ & 50658.2 & 0710.4 & $|-52.2|$ & -140.4 \\
\hline 30 & 17 & 120037 & 564 & 290 & 110 & 25.0 & 50851.3 & $|-26.3|$ & 50406.8 & 1459.0 & $|-52.2|$ & -209.1 \\
\hline 30 & 17 & 120045 & 566 & 290 & 115 & +2.8 & 50969.1 & $|-26.3|$ & 50619.2 & 50671.4 & $\mid-52.2$ & -161.8 \\
\hline 30 & 17 & 120053 & 568 & 290 & 120 & 228.6 & 51254.9 & -26.3 & 51063.8 & 1116.0 & $|-52.2|$ & -82.4 \\
\hline 30 & 17 & 120101 & 570 & 290 & 125 & 77.6 & 51604.0 & $|-26.4|$ & 51363.2 & 115.5 & $|-52.3|$ & -107.2 \\
\hline 30 & 17 & 120109 & 572 & 290 & 130 & 51982.8 & 52009.2 & $|-26.4|$ & 51797.4 & 49.7 & $-52.3 \mid$ & -92.7 \\
\hline 30 & 17 & 120117 & 574 & 290 & 135 & 77.4 & 52803.8 & $|-26.4|$ & 52982.4 & 34.7 & $|-52.3|$ & 102.5 \\
\hline 30 & 17 & 120126 & 576 & 290 & 140 & 92.6 & 53419.1 & $|-26.5|$ & 54432.0 & 884.4 & $|-52.4|$ & 519.7 \\
\hline 30 & 17 & 120134 & 578 & 290 & 145 & 34.6 & 53121.1 & $|-26.5|$ & 53490.8 & 543.2 & $|-52.4|$ & 198.1 \\
\hline 30 & 17 & 120143 & 580 & 290 & 150 & 77.4 & 52303.9 & $|-26.5|$ & 52096.8 & 2149.2 & $-52.4 \mid$ & -90.3 \\
\hline 30 & 17 & 120151 & 582 & 290 & 155 & 6.6 & 51883.2 & $\mid-26.6$ & 51783.2 & 35.7 & $-52.5 \mid$ & -36.7 \\
\hline 30 & 17 & 159 & 584 & 290 & 160 & 78.4 & 51305.0 & $\mid-26.6$ & 51409.0 & 161.5 & $\mid-52.5$ & 65.3 \\
\hline 30 & 17 & 120207 & 586 & 290 & 165 & 50848.2 & 50874.8 & -26.6 & 50079.8 & 50132.3 & -52.5 & -384.2 \\
\hline 30 & 17 & 120225 & $588^{\circ}$ & 290 & 170 & 65.2 & 51091.9 & $|-26.7|$ & 7160.4 & 7213.0 & $|-52.6|$ & -21952.4 \\
\hline 30 & 17 & 120234 & 590 & 290 & 175 & 51027.0 & 51053.7 & $|-26.7|$ & 50769.0 & 50821.6 & -52.6 & -129.0 \\
\hline 30 & 17 & 120241 & 592 & 290 & 180 & 51458.0 & 51484.7 & -26.7 & $51336: 8$ & 51389.4 & -52.6 & -60.6 \\
\hline 30 & 17 & 120249 & 594 & 290 & 185 & 52123.0 & 52149.8 & $\mid-26.8$ & 52203.4 & 52256.1 & $\mid-52.7$ & 40.2 \\
\hline 30 & 17 & 120258 & 596 & 290 & 190 & 53063.6 & 53090.4 & $|-26.8|$ & 53812.8 & 53865.5 & $\mid-52.7$ & 374.6 \\
\hline 30 & 17 & 120306 & 598 & 290 & 195 & 53659.4 & 53686.2 & -26.8 & 54564.2 & 4616.9 & $|-52.7|$ & 452.4 \\
\hline 30 & 17 & 120313 & 600 & 290 & 200 & 48.2 & 52975.1 & $|-26.9|$ & 53393.2 & 3445.9 & $|-52.7|$ & 222.5 \\
\hline 30 & 17 & 120321 & 602 & 290 & 205 & 35.6 & 51962.5 & $|-26.9|$ & 52068.0 & 52120.8 & $\mid-52.8$ & 66.2 \\
\hline 30 & 17 & 120329 & 604 & 290 & 210 & 38.6 & 50995.5 & $|-26.9|$ & 50698.6 & 50751.4 & $\mid-52.8$ & -135.0 \\
\hline 30 & 17 & 120337 & 606 & 290 & 215 & 8.4 & 50695.3 & -26.9 & 50379.2 & 50432.0 & -52.8 & -144.6 \\
\hline 30 & 17 & 120345 & 608 & 290 & 220 & 19.4 & 50746.4 & -27.0 & 50561.2 & 50614.1 & $|-52.9|$ & -79.1 \\
\hline 30 & 17 & 120353 & 610 & 290 & 225 & 33.0 & 50880.0 & $|-27.0|$ & 50797.0 & 50849.9 & $\mid-52.9$ & -28.0 \\
\hline 30 & 17 & 120400 & 612 & 290 & 230 & 32.0 & 51019.0 & $|-27.0|$ & 50970.0 & 51022.9 & $|-52.9|$ & -11.0 \\
\hline 30 & 17 & 120411 & 614 & 290 & 235 & 51099.2 & 51126.3 & $|-27.1|$ & 51068.4 & 21.4 & -53.0 & -15.4 \\
\hline 30 & 17 & 120418 & 616 & 290 & 240 & 51169.8 & 51196.9 & -27.1 & 51161.2 & 51214.2 & $|-53.0|$ & -4.3 \\
\hline 30 & 17 & 120425 & 618 & 290 & 245 & 51224.4 & 51251.5 & $|-27.1|$ & 51228.2 & 51281.2 & $-53.0 \mid$ & 1.9 \\
\hline 30 & 17 & 120434 & 620 & 290 & 250 & 51268.4 & 51295.5 & $\mid-27.1$ & 51270.4 & 51323.4 & $|-53.0|$ & 1.0. \\
\hline 30 & 17 & 120539 & 622 & 290 & 255 & 51301.4 & 51328.8 & $|-27.4|$ & 51291.2 & 51344.5 & $|-53.3|$ & -5.1 \\
\hline 30 & 17 & 120547 & 624 & 290 & 26 & 51322.4 & 51349.8 & $|-27.4|$ & 51322.2 & 51375.5 & $|-53.3|$ & -0.1 \\
\hline 30 & 17 & 120555 & 626 & 290 & 265 & .51344 .2 & 51371.6 & -27.4 & 51330.8 & 51384.1 & $\mid-53.3$ & -6.7 \\
\hline ר & 17 & 120603 & 628 & 290 & 270 & 51358.0 & 51385.5 & $|-27.5|$ & 51344.2 & | 51397.6 & $|-53.4|$ & -6.9 \\
\hline
\end{tabular}




\begin{tabular}{|c|c|c|c|c|c|c|c|c|c|c|c|c|}
\hline Line & Day & Time & Sta. & $\mathbf{X}$ & $\mathbf{Y}$ & uncorr. & $\begin{array}{l}\text { (nT) } \\
\text { corr. }\end{array}$ & diff. & $\begin{array}{l}\text { BOT } \\
\text { uncorr. }\end{array}$ & $\begin{array}{l}\text { TOM (nT } \\
\text { corr. }\end{array}$ & diff. & $\begin{array}{c}\text { Vert.Grad. } \\
\text { (nT/ft) }\end{array}$ \\
\hline 30 & 17 & 120612 & 630 & 290 & 275 & 51365.6 & 51393.1 & -27.5 & 51357.4 & 51410.8 & $\mid-53.4$ & -4.1 \\
\hline 30 & 17 & 120620 & 632 & 290 & 280 & 51371.8 & 51399.3 & -27.5 & 51368.2 & 51421.6 & $\mid-53.4$ & -1.8 \\
\hline 30 & 17 & 120628 & 634 & 290 & 285 & 51382.6 & 51410.2 & -27.6 & 51375.4 & 51428.9 & -53.5 & -3.6 \\
\hline 30 & 17 & 120535 & 636 & 290 & 290 & 51386.8 & 51414.4 & $|-27.6|$ & 51381.8 & 51435.3 & $|-53.5|$ & -2.5 \\
\hline 30 & 17 & 120644 & 638 & 290 & 295 & 51393.0 & 51420.6 & $|-27.6|$ & 51387.2 & 51440.7 & $\mid-53.5$ & -2.8 \\
\hline 30 & 17 & 120651 & 640 & 290 & 300 & 51395.6 & 51423.2 & $\mid-27.6$ & 51393.0 & 51446.5 & $|-53.5|$ & -1.3 \\
\hline 30 & 17 & 120659 & 642 & 290 & 305 & 51400.0 & 51427.7 & $|-27.7|$ & 51398.2 & 51451.8 & $\mid-53.6$ & -0.9 \\
\hline 31 & 17 & 121649 & 644 & 280 & 310 & 51398.4 & 51428.2 & $|-29.8|$ & 51384.8 & 51440.5 & $|-55.7|$ & -6.8 \\
\hline 31 & 17. & 121657 & 646 & 280 & 305 & 51403.4 & 51433.2 & $|-29.8|$ & 51403.8 & 51459.5 & $|-55.7|$ & 0.2 \\
\hline 31 & 17 & 121704 & 648 & 280 & 300 & 51401.6 & 51431.5 & $|-29.9|$ & 51393.4 & 51449.2 & $\mid-55.8$ & -4.1 \\
\hline 31 & 17 & 121712 & 650 & 280 & 295 & 51394.8 & 51424.7 & $|-29.9|$ & 51389.8 & 51445.6 & $|-55.8|$ & -2.5 \\
\hline 31 & 17 & 121720 & 652 & 280 & 290 & 51390.2 & 51420.1 & $|-29.9|$ & 51381.6 & 51437.4 & $\mid-55.8$ & -4.3 \\
\hline 31 & 17 & 121727 & 654 & 280 & 285 & 51382.8 & 51412.7 & $\mid-29.9$ & 51379.0 & 51434.9 & $|-55.9|$ & -1.9 \\
\hline 31 & 17 & 121735 & 656 & 280 & 280 & $51375: 4$ & 51405.4 & $-30.0 \mid$ & 51368.6 & 51424.5 & -55.9 & -3.4 \\
\hline 31 & 17 & 121743 & 658 & 280 & 275 & 51363.0 & 51393.0 & $|-30.0|$ & 51359.0 & 51414.9 & $|-55.9|$ & -2.0 \\
\hline 31 & 17 & 121750 & 660 & 280 & 270 & 51350.8 & 51380.8 & -30.0 & 51343.8 & 51399.7 & -55.9 & -3.5 \\
\hline 31 & 17 & 121757 & 662 & 280 & 265 & 51346.0 & 51376.1 & $-30.1 \mid$ & 51338.0 & 51394.0 & $|-56.0|$ & -4.0 \\
\hline 31 & 17 & 121805 & 664 & 280 & 260 & 51333.2 & 51363.3 & -30.1 & 51329.2 & 51385.2 & -56.0 & -2.0 \\
\hline 31 & 17 & 121813 & 666 & 280 & 255 & 51307.8 & 51337.9 & -30.1 & 51314.2 & 51370.2 & $-56.0 \mid$ & 3.2 \\
\hline 31 & 17 & 121820 & 668 & 280 & 250 & 51272.4 & 51302.5 & -30.1 & 51277.8 & 51333.8 & -56.0 & 2.7 \\
\hline 31 & 17 & 121835 & 670 & 280 & 245 & 51221.8 & 51252.0 & $\mid-30.2$ & 51227.8 & 51283.9 & $-56.1 \mid$ & 3.0 \\
\hline 31 & 17 & 121851 & 672 & 280 & 240 & 51172.0 & 51202.3 & -30.3 & 51171.4 & 51227.6 & -56.2 & -0.3 \\
\hline 31 & 17 & 121900 & 674 & 280 & 235 & 51082.4 & 51112.7 & -30.3 & 51079.0 & 51135.2 & -56.2 & -1.7 \\
\hline 31 & 17 & 121907 & 676 & 280 & 230 & 50960.8 & 50991.1 & -30.3 & 50944.6 & 51000.8 & -56.2 & \\
\hline 31 & 17 & 121915 & 678 & 280 & 225 & 50790.4 & 50820.7 & -30.3 & 50727.8 & 50784.0 & -56.2 & -31.3 \\
\hline 31 & 17 & 121923 & 680 & 280 & 220 & 50567.0 & 50597.4 & $|-30.4|$ & 50388.0 & 50444.3 & -56.3 & -89.5 \\
\hline 31 & 17 & 121934 & 682 & 280 & 215 & 50428.4 & 50458.8 & $|-30.4|$ & 50038.6 & 50094.9 & $|-56.3|$ & -194.9 \\
\hline 31 & 17 & 121942 & 684 & 280 & 210 & $50628.0^{\circ}$ & 50658.4 & -30.4 & 50118.4 & $50174: 7$ & -56.3 & -254.8 \\
\hline 31 & 17 & 121951 & 686 & 280 & 205 & 51638.8 & 51669.3 & -30.5 & 51029.8 & 51086.2 & $|-56.4|$ & -304.5 \\
\hline 31 & 17 & 121959 & 688 & 280 & 200 & 53467.6 & 53498.1 & -30.5 & 52083.0 & 52139.4 & -56.4 & -692.3 \\
\hline 31 & 17 & 122007 & 690 & 280 & 195 & 54127.6 & 54158.1 & -30.5 & 58546.2 & 58602.6 & -56.4 & 2209.3 \\
\hline 31 & 17 & 122015 & 692 & 280 & 190 & 52808.2 & 52838.8 & -30.6 & 53135.6 & 53192.1 & -56.5 & 163.7 \\
\hline 31 & 17 & 122024 & 694 & 280 & 185 & 51684.2 & 51714.8 & -30.6 & 51546.4 & 51602.9 & -56.5 & -68.9 \\
\hline 31 & 17 & 122031 & 696 & 280 & 180 & 51180.0 & 51210.6 & -30.6 & 51019.6 & 51076.1 & -56.5 & -80.2 \\
\hline 31 & 17 & 122039 & 698 & 280 & 175 & 50866.2 & 50896.8 & -30.6 & 50701.6 & 50758.1 & -56.5 & .82 .3 \\
\hline 31 & 17 & 122048 & 700 & 280 & 170 & 50646.8 & 50677.5 & -30.7 & 50411.8 & 50468.4 & -56.6 & -117.5 \\
\hline 31 & 17 & 122056 & 702 & 280 & 165 & 50596.0 & 50626.7 & $-30.7 \mid$ & 50299.8 & 50356.4 & -56.6 & -148.1 \\
\hline 31 & 17 & 122103 & 704 & 280 & 160 & 50662.4 & 50693.1 & -30.7 & 50487.0 & 50543.6 & -56.6 & -87.7 \\
\hline 31 & 17 & 122111 & 706 & 280 & 155 & 50809.6 & 50840.4 & $\mid-30.8$ & 50372.8 & 50429.5 & $-56.7 \mid$ & -218.4 \\
\hline 31 & 17 & 122119 & 708 & 280 & 150 & 51486.6 & 51517.4 & -30.8 & 51186.8 & 51243.5 & -56.7 & '-149.9 \\
\hline 31 & 17 & 122127 & 710 & 280 & 145 & 52641.2 & 52672.0 & -30.8 & 53062.0 & 53118.7 & -56.7 & 210.4 \\
\hline 31 & 17 & 122135 & 712 & 280 & 140 & 53178.6 & 53209.4 & -30.8 & 54259.4 & 54316.1 & -56.7 & 540.4 \\
\hline 31 & 17 & 122143 & 714 & 280 & 135 & 52649.4 & 52680.3 & -30.9 & 52823.2 & 52880.0 & -56.8 & 86.9 \\
\hline 31 & 17 & 122151 & 716 & 280 & 130 & 51967.8 & 51998.7 & $|-30.9|$ & 51857.6 & 51914.4 & -56.8 & -55.1 \\
\hline 31 & 17 & 122159 & 718 & 280 & 125 & 51624.0 & 51654.9 & -30.9 & 51547.6 & 51604.4 & -56.8 & -38.2 \\
\hline 31 & 17 & 122206 & 720 & 280 & 120 & 51456.0 & 51487.0 & -31.0 & 51429.2 & 51486.1 & -56.9 & -13.4 \\
\hline 31 & 17 & 122213 & 722 & 280 & 115 & 51343.2 & 51374.2 & -31.0 & 51379.6 & 51436.5 & -56.9 & 18.2 \\
\hline 31 & 17 & 122220 & 724 & 280 & 110 & 51234.8 & 51265.8 & -31.0 & 51224.4 & 51281.3 & $|-56.9|$ & -5.2 \\
\hline 1 & 17 & 122228 & 726 & 280 & 105 & 51191.4 & 51222.4 & $|-31.0|$ & 51147.6 & 51204.5 & $|-56.9|$ & -21.9 \\
\hline
\end{tabular}




\begin{tabular}{|c|c|c|c|c|c|c|c|c|c|c|c|c|}
\hline & & & & & & & SP (nT) & & BO & Ol & & ert.Gr \\
\hline Line & Day & Time & Sta.' & $x$ & $\mathbf{Y}$ & uncorr. & corr. & diff. & uncorr. & corr. & diff. & (nT/ \\
\hline 31 & 17 & 122235 & 728 & 280 & 100 & 51216.0 & 51247.1 & -31.1 & 51166.4 & 51223.4 & -57.0 & -24.8 \\
\hline 31 & 17 & 122242 & 730 & 280 & 95 & 51235.6 & 51266.7 & -31.1 & 51209.2 & 51266.2 & -57.0 & -13.2 \\
\hline 31 & 17 & 122250 & 732 & 280 & 90 & 51277.2 & 51308.3 & -31.1 & 51256.0 & 51313.0 & -57.0 & -10.6 \\
\hline 31 & 17 & 122256 & 734 & 280 & 85 & 51300.6 & 51331.7 & -31.1 & 51294.6 & 51351.6 & $-57.0 \mid$ & -3.0 \\
\hline 31 & 17 & 122303 & 736 & 280 & 80 & 51317.4 & 51348.6 & -31.2 & 51313.6 & 51370.7 & -57.1 & -1.8 \\
\hline 31 & 17 & 122311 & 738 & 280 & 75 & 51329.2 & 51360.4 & $\mid-31.2$ & 51330.6 & 51387.7 & -57.1 & 0.7 \\
\hline 31 & 17 & 122318 & 740 & 280 & 70 & 51333.6 & 51364.8 & -31.2 & 51337.2 & 51394.3 & -57.1 & 1.8 \\
\hline 31 & 17 & 122325 & 742 & $280 \mid$ & 65 & 51333.0 & 51364.2 & -31.2 & 51335.0 & 51392.1 & -57.1 & 1.0 \\
\hline 31 & $\cdot 17$ & 122333 & 744 & 280 & 60 & 51317.0 & 51348.3 & -31.3 & 51329.8 & 51387.0 & -57.2 & 6.4 \\
\hline 31 & 17 & 122442 & 746 & 280 & 55 & 51277.4 & 51308.9 & -31.5 & 51306.6 & 51364.0 & $|-57.4|$ & 14.6 \\
\hline 31 & 17 & 122449 & 748 & 280 & 50 & 51236.0 & 51267.6 & $\mid-31.6$ & 51276.8 & 51334.3 & $-57.5 \mid$ & 20.4 \\
\hline 31 & 17 & 122458 & 750 & 280 & 45 & 51167.2 & 51198.8 & -31.6 & 51220.2 & 51277.7 & -57.5 & 26.5 \\
\hline 31 & 17 & 122505 & 752 & 280 & 40 & 51090.8 & 51122.4 & -31.6 & 51132.0 & 51189.5 & -57.5 & 20.6 \\
\hline 31 & 17 & 122513 & 754 & 280 & 35 & 51054.2 & 51085.8 & -31.6 & 51079.2 & 51136.7 & -57.5 & 12.5 \\
\hline 31 & 17 & 122521 & 756 & 280 & 30 & 51048.2 & 51079.9 & -31.7 & 51049.4 & 51107.0 & $-57.6 \mid$ & 0.6 \\
\hline 31 & 17 & 122529 & 758. & 280 & 25 & 51083.2 & 51114.9 & -31.7 & 51073.8 & 51131.4 & -57.6 & -4.7 \\
\hline 31 & 17 & 122537 & 760 & 280 & 20 & 51136.0 & 51167.7 & -31.7 & 51129.8 & 51187.4 & -57.6 & -3.1 \\
\hline 31 & 17 & 122546 & 762 & 280 & 15 & 51190.0 & 51221.8 & -31.8 & 51171.4 & 51229.1 & $|-57.7|$ & -9.3 \\
\hline 31 & 17 & 122554 & 764 & $280 \mid$ & 10 & 51242.4 & 51274.2 & -31.8 & 51232.2 & 51289.9 & $|-57.7|$ & -5.1 \\
\hline 31 & 17 & 122601 & 766 & 280 & 5 & 51273.6 & 51305.4 & -31.8 & 51272.4 & 51330.1 & -57.7 & -0.6 \\
\hline 31 & 17 & 122609 & 768 & 280 & 0 & 51300.0 & 51331.8 & -31.8 & 51299.4 & 51357.1 & -57.7 & -0.3 \\
\hline 32 & 17 & 123458 & $780^{\circ}$ & 270 & 0 & 51353.4 & 51383.0 & -29.6 & .51348 .4 & 51404.5 & $|-56.1|$ & -2.5 \\
\hline 32 & 17 & 123506 & 782 & 270 & 5 & 51326.4 & 51356.0 & -29.6 & 51334.2 & 1390.4 & $|-56.2|$ & 3.9 \\
\hline 32 & 17 & 123512 & 784 & 270 & 10 & 51316.0 & 51345.6 & -29.6 & 51310.2 & 51366.4 & $|-56.2|$ & -2.9 \\
\hline 32 & 17 & 123519 & 786 & 270 & 15 & $51296.8^{-}$ & 51326.4 & -29.6 & 51295.8 & 51352.0 & -56.2 & -0.5 \\
\hline 32 & 17 & 123526 & 788 & $270 \mid$ & 20 & 51277.0 & 51306.6 & -29.6 & 51276.0 & 51332.2 & $|-56.2|$ & -0.5 \\
\hline 32 & 17 & 123534 & 790 & 270 & 25 & 51251.6 & 51281.1 & -29.5 & 51226.4 & 51282.5 & $-56.1 \mid$ & -12.6 \\
\hline 32 & 17 & 123541 & 792 & 270 & 30 & 51254.6 & 51284.1 & -29.5 & 50944.8 & 51000.9 & $-56.1 \mid$ & -154.9 \\
\hline 32 & 17 & 123549 & 794 & 270 & 35 & 51121.8 & 51151.2 & -29.4 & 50924.0 & 50980.1 & $|-56.1|$ & -98.9 \\
\hline 32 & 17 & 123557 & 796 & $270 \mid$ & 40 & 51252.4 & 51281.8 & -29.4 & 51254.4 & 51310.4 & -56.0 & 1.0 \\
\hline 32: & 17 & 123605 & 798 & 270 & 45 & 51314.0 & 51343.4 & -29.4 & 51327.8 & 51383.8 & $|-56.0|$ & 6.9 \\
\hline 32 & 17 & 123612 & 800 & 270 & 50 & 51339.4 & 51368.7 & -29.3 & 51347.2 & 51403.2 & -56.0 & 3.9 \\
\hline 32 & 17 & 123620 & 802 & 270 & 55 & 51356.6 & 51385.9 & -29.3 & 51359.8 & 51415.8 & $|-56.0|$ & 1.6 \\
\hline 32 & 17 & 123627 & 804 & 270 & 60 & 51365.4 & 51394.7 & -29.3 & 51362.4 & 51418.3 & $|-55.9|$ & -1.5 \\
\hline 32 & 17 & 123635 & 806 & 270 & 65 & 51367.4 & 51396.6 & -29.2 & 51361.0 & 51416.9 & -55.9 & -3.2 \\
\hline 32 & 17 & 123643 & 808 & 270 & 70 & 51366.8 & 51396.0 & -29.2 & 51356.0 & 51411.9 & -55.9 & -5.4 \\
\hline 32 & 17 & 123651 & 810 & $270 \mid$ & 75 & 51367.2 & 51396.3 & -29.1 & .51367 .8 & 51423.7 & -55.9 & 0.3 \\
\hline 32 & 17 & 123658 & 812 & 270 & 80 & 51358.2 & 51387.3 & -29.1 & 51353.6 & 51409.4 & -55.8 & -2.3 \\
\hline 32 & 17 & 123709 & 814 & 270 & 85 & 51350.4 & 51379.5 & -29.1 & 51342.8 & $\mid 51398.6$ & $|-55.8|$ & -3.8 \\
\hline 32 & 17 & 123717 & 816 & 270 & 90 & 51345.2 & 51374.2 & -29.0 & 51353.4 & 51409.2 & $|-55.8|$ & 4.1 \\
\hline 32 & 17 & 123725 & 818. & 270 & 95 & 51340.2 & 51369.2 & -29.0 & 51318.6 & 51374.3 & -55.7 & -10.8 \\
\hline 32 & 17 & 123733 & 820 & 270 & -100 & 51343.4 & 51372.3 & -28.9 & 51324.4 & 51380.1 & -55.7 & -9.5 \\
\hline 32 & 17 & 123741 & 822 & 270 & 105 & 51360.4 & 51389.3 & $|-28.9|$ & 51343.4 & 51399.1 & $|-55.7|$ & -8.5 \\
\hline 32 & 17 & 123748 & 824 & 270 & 110 & 51390.8 & 51419.7 & -28.9 & 51364.8 & 51420.5 & -55.7 & -13.0 \\
\hline 32 & 17 & 123756 & 826 & 270 & 115 & 51436.0 & 51464.8 & -28.8 & 51396.2 & 51451.8 & -55.6 & -19.9 \\
\hline 32 & 17 & 123803 & 828 & 270 & .120 & 51506.8 & 51535.6 & -28.8 & 51445.8 & 51501.4 & -55.6 & -30.5 \\
\hline 32 & 17 & 123812 & 830 & 270 & 125 & 51643.4 & 51672.2 & -28.8 & 51540.4 & 51596.0 & -55.6 & -51.5 \\
\hline 32 & 17 & 123819 & 832 & 270 & 130 & 51951.6 & 51980.3 & -28.7 & 51819.4 & 51875.0 & -55.6 & -66.1 \\
\hline 32 & 17 & $\mid 123828$ & 834 & 270| & 135 & 52537.6 & 52566.3 & $|-28.7|$ & 52708.2 & $|52763.7|$ & $|-55.5|$ & 85.3 \\
\hline
\end{tabular}




\begin{tabular}{|c|c|c|c|c|c|c|c|c|c|c|c|c|}
\hline Line & Jay & Tim & Sta. & $\mathbf{X}$ & $\mathbf{Y}$ & uncorr. & $\begin{array}{l}\text { (nT) } \\
\text { corr. }\end{array}$ & diff. & $\begin{array}{r}\text { BO' } \\
\text { uncorr. }\end{array}$ & $\begin{array}{l}\text { TOM (nT } \\
\text { corr. }\end{array}$ & diff. & $\begin{array}{l}\text { Vert.Grad. } \\
\text { (nT/ft) }\end{array}$ \\
\hline 32 & 17 & 123839 & 836 & 270 & 140 & 52911.0 & 52939.6 & -28.6 & 53591.2 & 53646.7 & -55.5 & 340.1 \\
\hline 32 & 17 & 123848 & 838 & 270 & 145 & 52147.8 & 52176.4 & -28.6 & 52371.2 & 52426.7 & -55.5 & 111.7 \\
\hline 32 & 17 & 123856 & 840 & 270 & 150 & 50875.6 & 50904.1 & -28.5 & 49843.8 & 49899.2 & -55.4 & -515.9 \\
\hline 32 & 17 & 123904 & 842 & 270 & 155 & 50519.8 & 50548.3 & -28.5 & 49896.6 & 49952.0 & -55.4 & -311.6 \\
\hline 32 & 17 & 123911 & 844 & 270 & 160 & 50648.6 & 50677.1 & -28.5 & 50542.6 & 50598.0 & $|-55.4|$ & -53.0 \\
\hline 32 & 17 & 123919 & 846 & 270 & 165 & 50591.2 & 50619.6 & -28.4 & 50327.6 & 50383.0 & $|-55.4|$ & -131.8 \\
\hline 32 & 17 & 123927 & 848 & 270 & 170 & 50635.0 & 50663.4 & $\mid-28.4$ & 50441.8 & 50497.1 & $|-55.3|$ & -96.6 \\
\hline 32 & 17 & 123934 & 850 & 270 & 175 & 50773.6 & 50802.0 & -28.4 & $\cdot 50646.2$ & 50701.5 & $-55.3 \mid$ & -63.7 \\
\hline 32 & 17 & 123942 & 852 & 270 & 180 & 50936.4 & 50964.7 & $\mid-28.3$ & 50860.8 & 50916.1 & $-55.3 \mid$ & -37.8 \\
\hline 32 & 17 & 123949 & 854 & 270 & 185 & 51072.6 & 51100.9 & $\mid-28.3$ & 51049.8 & 51105.1 & $|-55.3|$ & -11.4 \\
\hline 32 & 17 & 123957 & 856 & 270 & 190 & 51174.0 & 51202.3 & $\mid-28.3$ & 51020.8 & 076.0 & -55.2 & -76.6 \\
\hline 32 & 17 & 124004 & 858 & 270 & 195 & 51212.6 & 51240.8 & $\mid-28.2$ & 51069.8 & 51125.0 & -55.2 & -71.4 \\
\hline 32. & 17 & 124012 & 860 & 270 & 200 & 51135.2 & 51163.4 & -28.2 & 51152.8 & 51208.0 & -55.2 & 8.8 \\
\hline 32 & 17 & 124020 & 862 & 270 & 205 & 50862.4 & 50890.5 & $|-28.1|$ & 50926.2 & 50981.4 & $-55.2 \mid$ & 31.9 \\
\hline 32 & 17 & 124028 & 864 & 270 & 210 & 50341.2 & 50369.3 & $|-28.1|$ & 49863.8 & 49918.9 & $|-55.1|$ & -238.7 \\
\hline 32 & 17 & 124036 & 866 & 270 & 215 & 50227.2 & 50255.3 & -28.1 & 49783.6 & 49838.7 & -55.1 & -221.8 \\
\hline 32 & 17 & 124044 & 868 & 270 & 220 & 50505.4 & 50533.4 & -28.0 & 50310.2 & 50365.3 & $-55.1 \mid$ & -97.6 \\
\hline 32 & 17 & 124052 & 870 & 270 & 225 & 50770.0 & 50798.0 & -28.0 & 50706.4 & 50761.4 & $-55.0 \mid$ & -31.8 \\
\hline 32 & 17 & 124100 & 872 & 270 & 230 & 50983.4 & 51011.4 & $|-28.0|$ & 50955.4 & 51010.4 & $|-55.0|$ & -14.0 \\
\hline 32 & 17 & 124108 & 874 & 270 & 235 & 51110.4 & 51138.3 & -27.9 & 51107.6 & 51162.6 & -55.0 & -1.4 \\
\hline 32 & 17 & 124116 & 876 & 270 & 240 & 51193:0 & $51220: 9$ & -27.9 & 51193.6 & 51248.6 & -55.0 & 0.3 \\
\hline 32 & 17 & 124125 & 878 & 270 & 245 & 51251.8 & 51279.6 & $\mid-27.8$ & 51261.2 & 51316.1 & $|-54.9|$ & 4.7 \\
\hline 32 & 17. & 124141 & 880 & 270 & 250 & 51293.2 & 51321.0 & $\mid-27.8$ & 51286.6 & 51341.5 & -54.9 & -3.3 \\
\hline 32 & 17 & 125530 & 882 & 270 & 255 & 51328.8 & 51352.6 & $|-23.8|$ & 51333.8 & 51385.9 & $|-52.1|$ & 2.5 \\
\hline 32 & 17 & 125538 & 884 & 270 & 260 & 51375.8 & 51399.6 & $\mid-23.8$ & 51394.6 & 51446.6 & -52.0 & 9.4 \\
\hline 32 & 17 & 125546 & 886 & 270 & 265 & 51374.2 & 51397.9 & $|-23.7|$ & 51432.4 & 51484.4 & -52.0 & 29.1 \\
\hline 32 & 17 & 125554 & 888 & 270 & 270 & 51304.6 & 51328.3 & $|-23.7|$ & 51193.6 & 51245.6 & -52.0 & -55.5 \\
\hline 32 & 17 & 125602 & 890 & 270 & 275 & 51367.0 & 51390.7 & $|-23.7|$ & 51362.0 & 51413.9 & -51.9 & -2.5 \\
\hline 32 & 17 & 125609 & 892 & 270 & 280 & 51385.4 & 51409.0 & -23.6 & 51381.4 & 51433.3 & -51.9 & -2.0 \\
\hline 32 & 17 & 125617 & 894 & 270 & 285 & 51393.4 & 51417.0 & $\mid-23.6$ & 51391.2 & 51443.1 & -51.9 & -1.1 \\
\hline 32 & 17 & 125624 & 896 & 270 & 290 & 51398.2 & 51421.7 & -23.5 & 51396.8 & 51448.7 & -51.9 & -0.7 \\
\hline 32 & 17 & 125631 & 898 & 270 & 295 & 51402.2 & 51425.7 & -23.5 & 51403.0 & 51454.8 & -51.8 & 0.4 \\
\hline 32 & 17 & 125640 & 900 & 270 & 300 & 51406.2 & 51429.7 & $|-23.5|$ & 51403.8 & 51455.6 & -51.8 & -1.2 \\
\hline 32 & 17 & 125648 & 902 & 270 & 305 & 51411.4 & 51434.8 & $|-23.4|$ & 51394.8 & 51446.6 & -51.8 & -8.3 \\
\hline 32 & 17 & 125655 & 904 & 270 & 310 & 51411.4 & 51434.8 & $|-23.4|$ & 51391.2 & 51443.0 & -51.8 & -10.1 \\
\hline 33 & 17 & 130233 & 906 & 260 & 320 & 51418.6 & 51440.4 & $|-21.8|$ & 51421.6 & 51472.2 & -50.6 & 1.5 \\
\hline 33 & 17 & 130241 & 908 & 260 & 315 & 51416.8 & 51438.5 & $\mid-21.7$ & 51420.2 & 51470.8 & -50.6 & 1.7 \\
\hline 33 & 17 & 130248 & 910 & 260 & $310^{\circ}$ & 51413.6 & 51435.3 & $|-21.7|$ & 51420.2 & 51470.8 & -50.6 & 3.3 \\
\hline 33 & 17 & 130255 & 912 & 260 & 305 & .51409 .0 & 51430.7 & $|-21.7|$ & 51412.0 & 51462.5 & -50.5 & 1.5 \\
\hline 33 & 17 & 130302 & 914 & 260 & 300 & 51406.6 & 51428.2 & $\mid-21.6$ & 51408.2 & 51458.7 & -50.5 & 0.8 \\
\hline 33 & 17 & 130309 & 916 & 260 & 295 & 51401.8 & 51423.4 & $\mid-21.6$ & 51405.2 & 51455.7 & -50.5 & 1.7 \\
\hline 33 & 17 & 130316 & 918 & 260 & 290 & 51396.8 & 51418.4 & $\mid-21.6$ & 51399.4 & 51449.9 & $|-50.5|$ & 1.3 \\
\hline 33 & 17 & 130323 & 920 & 260 & 285 & 51391.4 & 51412.9 & $|-21.5|$ & 51390.4 & 51440.8 & $-50.4 \mid$ & -0.5 \\
\hline 33 & 17 & 130330 & 922 & 260 & 280 & 51385.6 & 51407.1 & $\mid-21.5$ & 51392.2 & 51442.6 & $|-50.4|$ & 3.3 \\
\hline 33 & 17 & 130340 & 924 & 260 & 275 & 51377.8 & 51399.3 & $|-21.5|$ & 51383.2 & 51433.6 & $|-50.4|$ & 2.7 \\
\hline 33 & 17 & 130347 & 926 & 260 & 270 & 51366.0 & 51387.4 & $|-21.4|$ & 51374.0 & 51424.4 & -50.4 & 4.0 \\
\hline 33 & 17 & 130354 & 928 & 260 & 265 & 51354.0 & 51375.4 & $\mid-21.4$ & 51359.4 & 51409.7 & -50.3 & 2.7 \\
\hline 33 & 17 & 130401 & 930 & 260 & 260 & 51337.6 & 51359.0 & $\mid-21.4$ & 51342.0 & 51392.3 & $|-50.3|$ & 2.2 \\
\hline 33 & 17 & 130409 & 932 & $260 \mid$ & 255 & 51313.0 & 51334.3 & $\mid-21.3$ & 51324.0 & 51374.3 & $\mid-50.3$ & 5.5 \\
\hline
\end{tabular}




\begin{tabular}{|c|c|c|c|c|c|c|c|c|c|c|c|c|}
\hline \multirow{2}{*}{\multicolumn{2}{|c|}{ Line |Day| }} & \multirow[b]{2}{*}{ Time } & \multirow[b]{2}{*}{ Sta. } & \multirow[b]{2}{*}{$\mathbf{x}$} & \multirow[b]{2}{*}{$\mathbf{Y}$} & \multirow{2}{*}{\multicolumn{2}{|c|}{ TOP (nT) }} & \multirow[b]{2}{*}{ diff. } & \multicolumn{2}{|c|}{ BOTTOM (nT } & \multirow[b]{2}{*}{ diff. } & \multirow{2}{*}{$\begin{array}{c}\text { Vert.Grad. } \\
\text { (nT/ft) }\end{array}$} \\
\hline & & & & & & & & & uncorr. & corr. & & \\
\hline 33 & 17 & 130416 & $\overline{934}$ & 260 & 250 & 51286.2 & 51307.5 & -21.3 & 51296.2 & 51346.5 & -50.3 & 5.0 \\
\hline 33 & 17 & 30424 & 936 & 260 & 245 & 51246.6 & 51267.9 & -21.3 & 51255.4 & 51305.6 & -50.2 & 4.4 \\
\hline 33 & 17 & 130431 & 938 & 260 & 240 & 51192.0 & 51213.2 & $\mid-21.2$ & 51194.4 & 51244.6 & -50.2 & 1.2 \\
\hline 33 & 17 & 130441 & 940 & 260 & 235 & 51104.4 & 51125.6 & -21.2 & 51115.8 & 51166.0 & -50.2 & 5.7 \\
\hline 33 & 17 & 130448 & 942 & 260 & 230 & 50970.8 & 50991.9 & -21.1 & 50967.0 & 51017.2 & -50.2 & -1.9 \\
\hline 33 & 17 & 130456 & 944 & 260 & 225 & 50767.6 & 50788.7 & -21.1 & 50700.8 & 50750.9 & -50.1 & -33.4 \\
\hline 33 & 17 & 130504 & 946. & 260 & 220 & 50474.4 & 50495.5 & -21.1 & 50267.8 & 50317.9 & -50.1 & -103.3 \\
\hline 33 & 17 & 130511 & 948 & 260 & 215 & 50189.8 & 50210.8 & -21.0 & 49715.4 & 49765.5 & -50.1 & -237.2 \\
\hline 33 & 17 & 130519 & 950 & 260 & 210 & 50134.0 & 50155.0 & -21.0 & $49686.0^{\circ}$ & 49736.1 & -50.1 & -224.0 \\
\hline 33 & 17 & 130527 & 952 & 260 & 205 & 50342.6 & 50363.6 & -21.0 & 499896.8 & 49946.8 & -50.0 & -222.9 \\
\hline 33 & 17 & 130535 & 954 & 260 & 200 & 50614.2 & 50635.1 & -20.9 & $50 \dot{0} 324.6$ & 50374.6 & -50.0 & -144.8 \\
\hline 33 & 17 & 130542 & 956 & 260 & 195 & 50803.6 & 50824.5 & -20.9 & 50608.8 & 50658.8 & -50.0 & -97.4 \\
\hline 33 & 17 & 130550 & 958 & 260 & 190 & 50892.0 & 50912.8 & -20.8 & 50767.8 & 0817.7 & -49.9 & -62.1 \\
\hline 33 & 17 & 130556 & 960 & 260 & 185 & 50882.2 & 50903.0 & -20.8 & 50808.0 & 50857.9 & -49.9 & -37.1 \\
\hline 33 & 17 & 130603 & 962 & 260 & 180 & 50815.0 & 50835.8 & -20.8 & 50736.2 & 50786.1 & -49.9 & -39.4 \\
\hline 33 & 17 & 130610 & 964 & 260 & 175 & 50716.6 & 50737.4 & -20.8 & 50588.0 & 50637.9 & -49.9 & -64.3 \\
\hline 33 & 17 & 130618 & 966 & 260 & 170 & 50663.0 & 50683.7 & -20.7 & 50440.4 & 50490.3 & -49.9 & -111.3 \\
\hline 33 & 17 & 130625 & 968 & 260 & 165 & 50826.8 & 50847.5 & -20.7 & 50646.6 & 50696.4 & -49.8 & -90.1 \\
\hline 33 & 17 & 130632 & 970 & 260 & 160 & 51131.8 & 51152.4 & -20.6 & 51132.0 & 1181.8 & -49.8 & 0.1 \\
\hline 33 & 17 & 130640 & 972 & 260 & 155 & 35.0 & 555.6 & -20.6 & 51706.8 & 51756.6 & -49.8 & 85.9 \\
\hline 33 & 17 & 130648 & 974 & 260 & 150 & 52049.8 & 52070.4 & -20.6 & 52208.2 & 52258.0 & -49.8 & 79.2 \\
\hline 33 & 17 & 130656 & 976 & $|260|$ & 145 & 52570.6 & |52591.1 & -20.5 & 52688.0 & 52737.7 & $\mid-49.7$ & 58.7 \\
\hline 33 & 17 & 130703 & 978 & 260 & 140 & 52821.8 & 52842.3 & -20.5 & 53999.4 & 54049.1 & -49.7 & 588.8 \\
\hline 33 & 17 & 130711 & 980 & 260 & 135 & 52108.0 & 52128.5 & -20.5 & 52065.6 & 52115.3 & -49.7 & -21.2 \\
\hline 33 & 17 & 130720 & 982 & 260 & 130 & 51683.4 & 51703.8 & -20.4 & 51580.8 & 1630.4 & -49.6 & -51.3 \\
\hline 33 & 17 & 130727 & 984 & 260 & 125 & 51530.2 & 51550.6 & -20.4 & 51461.8 & 1511.4 & -49.6 & -34.2 \\
\hline 33 & 17 & 130734 & 986 & 260 & 120 & 51.0 & 171.4 & -20.4 & 51397.8 & 1447.4 & -49.6 & -26.6 \\
\hline 33 & 17 & 130741 & 988 & 260 & 115 & 51410.0 & 430.3 & -20.3 & 51369.0 & 1418.6 & -49.6 & -20.5 \\
\hline 33 & 17 & 130751 & 990 & 260 & 110 & 51397.0 & 51417.3 & -20.3 & 51367.0 & 51416.5 & -49.5 & -15.0 \\
\hline 33 & 17 & 130758 & 992 & 260 & 105 & 51388.0 & 51408.2 & -20.2 & 51371.4 & 51420.9 & -49.5 & -8.3 \\
\hline 33 & 17 & 130806 & 994 & 260 & 100 & 51382.2 & 51402.4 & -20.2 & 51363.8 & 51413.3 & -49.5 & -9.2 \\
\hline 33 & 17 & 130814 & 996 & 260 & 95 & 51378.4 & 51398.6 & -20.2 & 51369.2 & 1418.7 & -49.5 & -4.6 \\
\hline 33 & 17 & 130822 & 998 & 260 & 90 & 51377.4 & 51397.5 & -20.1 & 51370.6 & 51420.0 & -49.4 & -3.4 \\
\hline 33 & 17 & 130829 & 1000 & 260 & 85 & 51379.6 & 51399.7 & -20.1 & 51379.0 & 51428.4 & -49.4 & -0.3 \\
\hline 33 & 17 & 130837 & 1002 & 260 & 80 & 51383.2 & 51403.3 & -20.1 & 51383.6 & 51433.0 & -49.4 & 0.2 \\
\hline 33 & 17 & 130845 & 1004 & 260 & 75 & 51386.0 & 51406.0 & -20.0 & 51377.2 & 51426.5 & -49.3 & -4.4 \\
\hline 33 & 17 & 130854 & 1006 & 260 & 70 & 51391.6 & 51411.6 & -20.0 & 51381.6 & 51430.9 & -49.3 & -5.0 \\
\hline 33 & 17 & 131140 & 1008 & 260 & 65 & 51388.6 & 51407.8 & -19.2 & 51395.4 & 51444.2 & -48.8 & 3.4 \\
\hline 33 & 17 & 131148 & 1010 & 260 & 60 & 37.2 & 51406.3 & $\mid-19.1$ & 51396.0 & 51444.7 & -48.7 & 4.4 \\
\hline 33 & 17 & 131155 & 1012 & 260 & 55 & 51384.4 & 51403.5 & -19.1 & 51397.0 & 51445.7 & -48.7 & 6.3 \\
\hline 33 & 17 & 131203 & 1014 & 260 & 50 & 51378.6 & 51397.7 & $\mid-19.1$ & 51371.0 & 51419.7 & -48.7 & -3.8 \\
\hline 33 & 17 & 131212 & 1016 & 260 & 45 & 51350.8 & 51369.8 & $\mid-19.0$ & 51363.6 & 51412.2 & -48.6 & 6.4 \\
\hline 33 & 17 & 131218 & 1018 & 260 & 40 & 51294.2 & 51313.2 & $\mid-19.0$ & 51319.0 & 5.1367 .6 & -48.6 & 12.4 \\
\hline 33 & 17 & 131225 & 1020 & 260 & 35 & 51148.0 & 51167.0 & $\mid-19.0$ & $51180: 8$ & 51229.4 & -48.6 & 16.4 \\
\hline 33 & 17 & 131232 & 1022 & 260 & 30 & 51211.2 & 51230.1 & $\mid-18.9$ & 51202.0 & 51250.6 & -48.6 & -4.6 \\
\hline 33 & 17 & 131240 & 1024 & 260 & 25 & 51311.0 & 51329.9 & $\mid-18.9$ & 51305.6 & 51354.2 & -48.6 & -2.7 \\
\hline 33 & 17 & 131247 & 1026 & 260 & 20 & 51351.0 & 51369.9 & -18.9 & 51349.6 & 51398.1 & -48.5 & -0.7 \\
\hline 33 & 17 & 131255 & 1028 & 260 & 15 & 51357.6 & 51376.4 & $\mid-18.8$ & 51361.2 & 51409.7 & -48.5 & 1.8 \\
\hline 33 & 17 & 131306 & 1030 & 260 & 10 & 51359.8 & 51378.6 & $\mid-18.8$ & 51359.6 & 51408.1 & -48.5 & -0.1 \\
\hline
\end{tabular}




\begin{tabular}{|c|c|c|c|c|c|c|c|c|c|c|c|c|}
\hline Line & Day & lime & sta. & $\mathbf{x}$ & $\mathbf{Y}$ & ncorr. & $\begin{array}{l}\text { (nT) } \\
\text { corr. }\end{array}$ & allt. & $\begin{array}{l}\text { BOT } \\
\text { ncorr. }\end{array}$ & $\begin{array}{l}\text { TOM (nT } \\
\text { corr. }\end{array}$ & diff. & $\begin{array}{l}\text { Vert.Grad. } \\
\text { (nT/ft) }\end{array}$ \\
\hline 33 & 17 & 131317 & 1032 & 260 & 5 & 51368.4 & 51387.1 & -18.7 & 51371.8 & 51420.2 & -48.4 & 1.7 \\
\hline 33 & 17 & 131327 & 1034 & 260 & 0 & 51375.6 & 51394.3 & -18.7 & 51372.2 & 51420.6 & -48.4 & -1.7 \\
\hline 34 & 17 & 132300 & 1046 & 250 & 0 & 51392.4 & 51412.6 & -20.2 & 51393.4 & 51443.1 & -49.7 & 0.5 \\
\hline 34 & 17 & 132310 & 1048 & $250 \mid$ & 5 & 51386.6 & 51406.6 & $|-20.0|$ & 51382.0 & 51431.6 & -49.6 & -2.3 \\
\hline 34 & 17 & 132330 & 1050 & 250 & 10 & 51388.2 & 51408.2 & -20.0 & 51389.4 & 51439.0 & -49.6 & 0.6 \\
\hline 34 & 17 & 132344 & 1052 & $250 \mid$ & 15 & 51390.6 & $51410: 7$ & $|-20.1|$ & 51380.8 & 51430.4 & -49.6 & -4.9 \\
\hline 34 & 17 & 132355 & 1054 & $250 \mid$ & 20 & 51388.2 & 51408.3 & $|-20.1|$ & 51381.8 & 51431.5 & -49.7 & -3.2 \\
\hline 34 & 17 & 132404 & 1056 & $250 \mid$ & 25 & 51384.4 & 51404.5 & $|-20.1|$ & 51379.8 & 51429.5 & -49.7 & -2.3 \\
\hline 34 & 17 & 132411 & 1058 & 250 & 30 & 51389.2 & 51409.4 & $\mid-20: 2$ & 51375.2 & 51424.9 & $\mid-49.7$ & -7.0 \\
\hline 34 & 17 & 132419 & 1060 & 250 & 35 & 51390.0 & 51410.2 & $\mid-20.2$ & 51377.0 & 51426.7 & -49.7 & -6.5 \\
\hline 34 & 17 & 132427 & 1062 & 250 & 40 & 51395.4 & 51415.6 & -20.2 & 51396.2 & 51446.0 & -49.8 & 0.4 \\
\hline 34 & 17 & 132436 & 1064 & 250 & 45 & 51398.8 & 51419.0 & -20.2 & 51393.2 & 51443.0 & -49.8 & -2.8 \\
\hline 34 & 17 & 132444 & 1066 & 250 & 50 & 51405.0 & 1425.3 & -20.3 & 51385.0 & 1434.8 & -49.8 & -10.0 \\
\hline 34 & 17 & 132452 & 1068 & 250 & 55 & 51402.8 & 51423.1 & -20.3 & 51384.4 & 1434.2 & $-49,8$ & -9.2 \\
\hline 34 & 17 & 132501 & 1070 & 250 & 60 & 51408.6 & 51428.9 & -20.3 & 51375.0 & 51424.8 & -49.8 & -16.8 \\
\hline 34 & 17 & 132508 & 1072 & 250 & 65 & 51405.2 & 51425.5 & -20.3 & 51403.0 & 51452.9 & -49.9 & -1.1 \\
\hline 34 & 17 & 132517 & 1074 & 250 & 70 & 51402.0 & 51422.4 & -20.4 & $51374.0^{\circ}$ & 51423.9 & -49.9 & -14.0 \\
\hline 34 & 17 & 132525 & 1076 & 250 & 75 & 51400.0 & 51420.4 & $\mid-20.4$ & 51380.8 & 51430.7 & -49.9 & -9.6 \\
\hline 34 & 17 & 132532 & 1078 & 250 & 80 & 51397.2 & 51417.6 & $\mid-20.4$ & 51376.8 & 51426.7 & -49.9 & -10.2 \\
\hline 34 & 17 & 132541 & 1080 & 250 & 85 & 51394.8 & 51415.2 & $|-20.4|$ & 51374.2 & 51424.1 & -49.9 & -10.3 \\
\hline 34 & 17 & 132549 & 1082 & 250 & 90 & 51394.4 & 51414.9 & $\mid-20.5$ & 51375.6 & $51425: 6$ & -50.0 & -9.4 \\
\hline 34 & 17 & 132558 & 1084 & 250 & 95 & 51396.0 & 51416.5 & $\mid-20.5$ & 51358.6 & 51408.6 & -50.0 & -18.7 \\
\hline 34 & 17 & 132606 & 1086 & 250 & 100 & 51457.2 & 51477.7 & -20.5 & 51340.4 & 51390.4 & -50.0 & -58.4 \\
\hline 34 & 17 & 132615 & 1088 & 250 & 105 & 51990.6 & 52011.1 & -20.5 & 9443.8 & 9493.8 & -50.0 & -21273.4 \\
\hline 34 & 17 & 132623 & 1090 & 250 & 110 & 51531.6 & 51552.2 & -20.6 & 51250.2 & 51300.3 & -50.1 & -140.7 \\
\hline 34 & 17 & 132631 & 1092 & 250 & 115 & 51430.6 & 51451.2 & -20.6 & 51341.8 & 1391.9 & -50.1 & -44.4 \\
\hline 34 & 17 & 132640 & 1094 & 250 & 120 & 51477.6 & 498.2 & -20.6 & 51386.4 & $436: 5$ & -50.1 & 45.6 \\
\hline 34 & 17 & 132647 & 1096 & 250 & 125 & 51597.4 & 51618.0 & $\mid-20.6$ & 51486.2 & 51536.3 & -50.1 & -55.6 \\
\hline 34 & 17 & 132655 & 1098 & 250 & 130 & 51851.6 & 51872.3 & -20.7 & 51666.4 & 51716.5 & $\mid-50.1$ & -92.6 \\
\hline 34 & 17 & 132703 & 1100 & 250 & 135 & 52348.0 & 52368.7 & $|-20.7|$ & 52479.2 & 52529.4 & -50.2 & 65.6 \\
\hline 34 & 17 & 132711 & 1102 & 250 & 140 & 52747.2 & 52767.9 & $\mid-20.7$ & 53256.2 & 53306.4 & -50.2 & 254.5 \\
\hline 34 & 17 & 132719 & 1104 & 250 & 145 & 52656.0 & 52676.7 & $|-20.7|$ & 53443.2 & 53493.4 & $\mid-50.2$ & 393.6 \\
\hline 34 & 17 & 132727 & 1106 & 250 & 150 & 51953.0 & 51973.8 & $|-20.8|$ & 52040.0 & 52090.2 & $\mid-50.2$ & 43.5 \\
\hline 34 & 17 & 132735 & 1108 & 250 & 155 & 51455.4 & 51476.2 & $\mid-20.8$ & 51530.6 & 51580.8 & -50.2 & 37.6 \\
\hline 34 & 17 & 132742 & 1110 & 250 & 160 & 51064.2 & 51085.0 & $\mid-20.8$ & 51158.4 & 1208.7 & $\mid-50.3$ & 47.1 \\
\hline 34 & 17 & 132750 & 1112 & 250 & 165 & 50682.0 & 50702.8 & $\mid-20.8$ & 50283.4 & 0333.7. & -50.3 & -199.3 \\
\hline 34 & 17 & 132758 & 1114 & 250 & 170 & 50736.0 & 50756.9 & -20.9 & 50556.2 & 50606.5 & -50.3 & -89.9 \\
\hline 34 & 17 & 132805 & 1116 & 250 & 175 & 50909.8 & 50930.7 & -20.9 & 50823.0 & 50873.3 & -50.3 & -43.4 \\
\hline 34 & 17 & 132813 & 1118 & 250 & 180 & 51057.6 & 51078.5 & -20.9 & 51005.0 & 51055.3 & -50.3 & -26.3 \\
\hline 34 & 17 & 132820 & 1120 & 250 & 185 & 51146.8 & 51167.7 & $\mid-20.9$ & 51106.6 & 51157.0 & \begin{tabular}{|l|}
-50.4 \\
\end{tabular} & -20.1 \\
\hline 34 & 17 & 132827 & 1122 & 250 & 190 & 51177.8 & 51198.7 & $\mid-20.9$ & 51078.0 & 51128.4 & -50.4 & -49.9 \\
\hline 34 & 17 & 132834 & 1124 & 250 & 195 & 51233.2 & 51254.2 & -21.0 & 51185.0 & 51235.4 & $\mid-50.4$ & -24.1 \\
\hline 34 & 17 & 132842 & 1126 & 250 & 200 & 51314.0 & 51335.0 & $\mid-21.0$ & 51430.0 & 51480.4 & -50.4 & 58.0 \\
\hline 34 & 17 & 132850 & 1128 & 250 & 205 & 51240.2 & 51261.2 & $\mid-21.0$ & 51369.2 & 51419.6 & $\mid-50.4$ & 64.5 \\
\hline 34 & 17 & 132858 & 1130 & 250 & 210 & 51011.2 & 51032.2 & $|-21.0|$ & 50896.6 & 50947.1 & -50.5 & -57.3 \\
\hline 34 & 17 & 132906 & 1132 & 250 & 215 & 50942.0 & 50963.1 & -21.1 & 50808.2 & 50858.7 & -50.5 & -66.9 \\
\hline 34 & 17 & 132914 & 1134 & 250 & 220 & 50995.6 & 51016.7 & -21.1 & 50954.0 & 51004.5 & -50.5 & -20.8 \\
\hline 34 & 17 & 132921 & 1136 & 250 & 225 & 51093.0 & 51114.1 & $\mid-21.1$ & 51074.4 & 51124.9 & -50.5 & -9.3 \\
\hline 34 & 17 & 132929 & 1138 & 250 & 230 & 51170.8 & 51191.9 & -21.1 & 51144.6 & 51195.1 & -50.5 & -13.1 \\
\hline
\end{tabular}




\begin{tabular}{|c|c|c|c|c|c|c|c|c|c|c|c|c|}
\hline & & & & & & & (nT) & & BOT & TOM (nT) & & \\
\hline Line & Day & Time & Sta. & $\mathbf{x}$ & $\mathbf{Y}$ & uncorr. & corr. & diff. & uncorr. & corr. & diff. & \\
\hline$\overline{34}$ & 17 & 132942 & 1140 & 250 & 235 & 51224.4 & 51245.6 & -21.2 & 51226.6 & 51277.2 & -50.6 & 1.1 \\
\hline 34 & 17 & 132949 & 1142 & $250 \mid$ & 240 & 51269.8 & 51291.0 & -21.2 & 51262.6 & 51313.2 & -50.6 & -3.6 \\
\hline 34 & 17 & 132956 & 1144 & 250 & 245 & 51300.4 & 51321.6 & -21.2 & 51281.0 & 51331.6 & -50.6 & -9.7 \\
\hline 34 & 17 & 133004 & 1146 & 250 & 250 & 51326.0 & 51347.2 & -21.2 & 51317.0 & 51367.6 & -50.6 & -4.5 \\
\hline 34 & 17 & 133117 & 1148 & 250 & 255 & 51342.2 & 51363.6 & -21.4 & 51307.4 & 51358.2 & -50.8 & -17.4 \\
\hline 34 & 17 & 133128 & 1150 & 250 & 260 & 51361.4 & 51382.9 & -21.5 & 51359.8 & 51410.6 & -50.8 & -0.8 \\
\hline 34 & 17 & 133137 & 1152 & 250 & 265. & 51372.2 & 51393.7 & -21.5 & 51363.8 & 51414.7 & -50.9 & -4.2 \\
\hline 34 & 17 & 133145 & 1154 & 250 & 270 & 51383.4 & 51404.9 & -21.5 & 51376.8 & 51427.7 & $\mid-50.9$ & -3.3 \\
\hline 34 & 17 & 133154 & 1156 & 250 & 275 & 51392.8 & 51414.4 & -21.6 & 51376.8 & 51427.7 & -50.9 & -8.0 \\
\hline 34 & 17 & 133202 & 1158 & 250 & 280 & 51401.8 & 51423.4 & -21.6 & 51392.2 & 51443.1 & -50.9 & -4.8 \\
\hline 34 & 17 & 133210 & 1160 & 250 & 285 & 51404.2 & 51425.8 & -21.6 & 51392.2 & 51443.1 & -50.9 & -6.0 \\
\hline 34 & 17 & 133218 & 1162 & $250 \mid$ & 290 & 51413.2 & 51434.8 & -21.6 & 51390.0 & 51441.0 & -51.0 & -11.6 \\
\hline 34 & 17 & 133226 & 1164 & 250 & 295 & 51415.8 & 51437.5 & $\mid-21.7$ & 51401.2 & 51452.2 & -51.0 & -7.3 \\
\hline 34 & 17 & 133233 & 1166 & 250 & 300 & 51420.0 & 51441.7 & -21.7 & 51407.4 & 51458.4 & -51.0 & -6.3 \\
\hline 34 & 17 & 133241 & 1168 & 250 & 305 & 51422.0 & 51443.7 & $|-21.7|$ & 51408.0 & 51459.0 & -51.0 & -7.0 \\
\hline 34 & 17 & 133249 & 1170 & 250 & 310 & 51417.4 & 51439.1 & -21.7 & 51414.0 & 51465.0 & -51.0 & -1.7 \\
\hline 34 & 17 & 133256 & 1172 & 250 & 315 & 51420.8 & 51442.5 & -21.7 & 51420.4 & 51471.5 & $-51: 1$ & -0.2 \\
\hline 35 & 17 & 133945 & 1174 & 240 & 325 & 51431.8 & 51454.8 & -23.0 & 51417.8 & 51469.9 & $\mid-52.1$ & -7.0 \\
\hline 35 & 17 & 133953 & 1176 & 240 & 320 & 51423.4 & 51446.4 & -23.0 & 51418.4 & 51470.5 & $\mid-52.1$ & -2.5 \\
\hline 35 & 17 & 133959 & 1178 & 240 & 315 & 51419.8 & 51442.8 & -23.0 & 51417.4 & 51469.6 & $\mid-52.2$ & -1.2 \\
\hline 35 & 17 & 134009 & 1180 & 240 & 310 & 51417.6 & 51440.7 & $-23: 1$ & 51414.8 & 51467.0 & -52.2 & -1.4 \\
\hline 35 & 17 & 134016 & 1182 & 240 & 305 & 51414.6 & 51437.7 & -23.1 & 51410.2 & 51462.4 & -52.2 & -2.2 \\
\hline 35 & 17 & 134023 & 1184 & 240 & 300 & 51409.6 & 51432.7 & -23.1 & 51406.6 & 51458.8 & $\mid-52.2$ & -1.5 \\
\hline 35 & 17 & 134031 & 1186 & 240 & 295 & 51408.4 & 51431.5 & $|-23.1|$ & 51399.8 & 452.0 & -52.2 & -4.3 \\
\hline 35 & 17 & 134037 & 1188 & 240 & 290 & 51402.6 & 51425.7 & $-23.1 \mid$ & 51401.0 & 51453.2 & -52.2 & -0.8 \\
\hline 35 & 17 & 134044 & 1190 & 240 & 285 & 51396.2 & 51419.4 & -23.2 & 51397.2 & 51449.5 & -52.3 & 0.5 \\
\hline 35 & 17 & 134052 & 1192 & 240 & 280 & 51390.2 & 51413.4 & -23.2 & 51385.8 & 51438.1 & -52.3 & -2.2 \\
\hline 35 & 17 & 134059 & 1194 & 240 & 275 & 51382.2 & 51405.4 & -23.2 & 51377.0 & 51429.3 & $\mid-52.3$ & -2.6 \\
\hline 35 & 17 & 134107 & 1196 & 240 & 270 & 51373.8 & 51397.0 & -23.2 & 51369.0 & 121.3 & $\mid-52.3$ & -2.4 \\
\hline 35 & 17 & 134114 & 1198 & 240 & 265 & 51361.0 & 51384.2 & -23.2 & 51359.6 & 411:9 & $\mid-52.3$ & -0.7 \\
\hline 35 & 17 & 134122 & 1200 & 240 & 260 & 51345.2 & 51368.5 & -23.3 & 51342.8 & 395.2 & $\mid-52.4$ & -1.2 \\
\hline 35 & 17 & 134129 & 1202 & 240 & 255 & 51330.2 & 51353.5 & -23.3 & 51323.8 & 51376.2 & |-52.4 & -3.2 \\
\hline 35 & 17 & 134143 & 1204 & 240 & 250 & 51309.8 & 51333.1 & -23.3 & 51302.6 & 51355.0 & $\mid-52.4$ & -3.6 \\
\hline 35 & 17 & 134152 & 1206 & 240 & 245 & 51277.8 & 51301.2 & -23.4 & 51268.2 & 51320.6 & -52.4 & -4.8 \\
\hline 35 & 17 & 134 & 1208 & 240 & 240 & 51242.0 & 51265.4 & $\mid-23: 4$ & 51239.6 & 51292.1 & -52.5 & -1.2 \\
\hline 35 & 17 & 134208 & 1210 & 240 & 235 & 51204.6 & 51228.0 & $\mid-23.4$ & 51195.4 & 51247.9 & -52.5 & -4.6 \\
\hline 35 & 17 & 134216 & 1212 & 240 & 230 & 51170.4 & 51193.8 & $\mid-23.4$ & 51142.0 & 51194.5 & -52.5 & -14.2 \\
\hline 35 & 17 & 134225 & 1214 & 240 & 225 & 51163.0 & 51186.5 & $\mid-23.5$ & 51109.8 & 51162.3 & -52.5 & -26.6 \\
\hline 35 & 17 & 134232 & 1216 & 240 & .220 & 51264.2 & 51287.7 & $\mid-23.5$ & 51182.8 & 51235.3 & -52.5 & -40.7 \\
\hline 35 & 17 & 134240 & 1218 & 240 & 215 & 51602.0 & 51625.5 & -23.5 & 51629.8 & 51682.4 & -52.6 & 13.9 \\
\hline 35 & 17 & 134247 & 1220 & 240 & 210 & 51983.4 & 52006.9 & -23.5 & 52205.2 & 52257.8 & -52.6 & 110.9 \\
\hline 35 & 17 & 134255 & 1222 & 240 & 205 & 52173.2 & 52196.7 & $\mid-23.5$ & 52255.4 & 52308.0 & -52.6 & 41.1 \\
\hline 35 & 17 & 134302 & 1224 & 240 & 200 & 52160.0 & 52183.6 & -23.6 & $52248: 4$ & 52301.0 & -52.6 & 44.2 \\
\hline 35 & 17 & 134309 & 1226 & 240 & 195 & 51942.4 & 51966.0 & $\mid-23.6$ & 51981.0 & 52033.6 & -52.6 & 19.3 \\
\hline 35 & 17 & 134316 & 1228 & 240 & 190 & 51642.6 & 51666.2 & $\mid-23.6$ & 51625.8 & 51678.5 & -52.7 & -8.4 \\
\hline 35 & 17 & 134323 & 1230 & 240 & 185 & 51367.0 & 51390.6 & -23.6 & 51332.6 & 51385.3 & -52.7 & -17.2 \\
\hline 35 & 17 & 134330 & 1232 & 240 & 180 & 51101.8 & 51125.5 & -23.7 & 51047.4 & 51100.1 & -52.7 & -27.2 \\
\hline 35 & 17 & 134337 & 1234 & 240 & 175 & 50835.2 & 50858.9 & -23.7 & 50703.0 & 50755.7 & $\mid-52.7$ & -66.1 \\
\hline 35 & 17 & 134344 & 1236 & 240 & 170 & 50616.6 & $\mid 50640.3$ & $\mid-23.7$ & 50342.6 & |50395.3 & $\mid-52.7$ & -137.0 \\
\hline
\end{tabular}




\begin{tabular}{|c|c|c|c|c|c|c|c|c|c|c|c|c|}
\hline Line & Day & Time & $t a$ & $x$ & $Y$ & ${ }^{T}$ & PP (nT) & diff. & $\mathrm{BO}^{7}$ & TOM (nT & if & Vert.Grad. \\
\hline 35 & 17 & 134352 & 1238 & 240 & 165 & 50616.8 & 50640.5 & -23.7 & 50270.8 & 50323.5 & -52.7 & -173.0 \\
\hline 35 & 17 & 134400 & 1240 & 240 & 160 & 50923.2 & 50946.9 & $|-23.7|$ & 50426.0 & 50478.8 & -52.8 & -248.6 \\
\hline 35 & 17 & 134408 & 1242 & 240 & 155 & .51719 .4 & 51743.2 & $|-23.8|$ & 51448.6 & 51501.4 & $\mid-52.8$ & -135.4 \\
\hline 35 & 17 & 134416 & 1244 & 240 & 150 & 52486.8 & 52510.6 & $|-23.8|$ & 52965.8 & 53018.6 & $\mid-52.8$ & 239.5 \\
\hline 35 & 17 & 134424 & 1246 & 240 & 145 & 52670.0 & 52693.8 & $\mid-23.8$ & 52945.2 & 52998.0 & $\mid-52.8$ & 137.6 \\
\hline 35 & 17 & 134433 & 1248 & 240 & 140 & 52431.6 & 52455.4 & $|-23.8|$ & 52679.0 & 52731.9 & -52.9 & 123.7 \\
\hline 35 & 17 & 134441 & 1250 & 240 & 135 & 51899.2 & 51923.1 & $|-23.9|$ & 51776.6 & 51829.5 & $\mid-52.9$ & $: 61.3$ \\
\hline 35 & 17 & 134450 & 1252 & 240 & 130 & 51626.0 & 51649.9 & $\mid-23.9$ & 51521.8 & 51574.7 & -52.9 & -52.1 \\
\hline 35 & 17 & 134459 & 1254 & 240 & 125 & 51503.4 & 51527.3 & $|-23.9|$ & 51434.8 & 51487.7 & -52.9 & -34.3 \\
\hline 35 & 17 & 134506 & 1256 & 240 & 120 & 51448.4 & 51472.3 & $|-23.9|$ & 51402.2 & 51455.1 & $\mid-52.9$ & -23.1 \\
\hline 35 & 17 & 134513 & 1258 & 240 & 115 & 51413.6 & 51437.6 & $|-24.0|$ & 51376.6 & 51429.6 & -53.0 & -18.5 \\
\hline 35 & 17 & 134522 & 1260 & 240 & 110 & 51324.0 & 51348.0 & $|-24.0|$ & 51400.8 & 51453.8 & $\mid-53.0$ & 38.4 \\
\hline 35 & 17 & 134530 & 1262 & 240 & 105 & 51388.8 & 51412.8 & $|-24.0|$ & 51358.8 & 51411.8 & $\mid-53.0$ & -15.0 \\
\hline 35 & 17 & 134537 & 1264 & 240 & 100 & 51392.6 & 51416.6 & -24.0 & 51374.8 & 51427.8 & -53.0 & -8.9 \\
\hline 35 & 17 & 134545 & 1266 & 240 & 95 & 51400.4 & 51424.5 & $|-24.1|$ & 51383.2 & 51436.2 & -53.0 & -8.6 \\
\hline 35 & 17 & 134551 & 1268 & 240 & 90 & 51398.8 & 51422.9 & $|-24.1|$ & 51381.0 & |51434.1 & $\mid-53.1$ & -8.9 \\
\hline 35 & 17 & 134558 & 1270 & 240 & 85 & 51395.8 & 51419.9 & $|-24.1|$ & 51386.6 & 51439.7 & $|-53.1|$ & -4.6 \\
\hline 35 & 17 & 134609 & 1272 & 240 & 80 & 51404.0 & 51428.1 & $|-24.1|$ & 51381.6 & 51434.7 & $|-53.1|$ & -11.2 \\
\hline 35 & 17 & 134920 & 1274 & 240 & 75 & 51399.2 & 51423.9 & $|-24.7|$ & 51384.4 & 51438.0 & -53.6 & -7.4 \\
\hline 35 & 17 & 134929 & 1276 & 240 & 70 & 51395.8 & 51420.5 & $|-24.7|$ & 51395.4 & 51449.0 & -53.6 & -0.2 \\
\hline 35 & 17 & 134937 & 1278 & 240 & 65 & 51403.6 & 51428.4 & $|-24.8|$ & 51392.0 & 51445.6 & $\mid-53.6$ & -5.8 \\
\hline 35 & 17 & 134946 & 1280 & 240 & 60 & 51401.0 & $51425: 8$ & $|-24.8|$ & 51393.4 & 51447.1 & $|-53.7|$ & -3.8 \\
\hline 35 & 17 & 135002 & 1282 & 240 & 55 & 51398.0 & 51422.8 & $\mid-24.8$ & 51403.2 & 51456.9 & $\mid-53.7$ & 2.6 \\
\hline 35 & 17 & 135011 & 1284 & 240 & 50 & 51411.0 & 51435.9 & $\mid-24.9$ & 51409.6 & 51463.3 & $\mid-53.7$ & -0.7 \\
\hline 35 & 17. & 135020 & 1286 & 240 & 45 & 51428.6 & 51453.5 & $\mid-24.9$ & 51424.4 & 51478.2 & $\mid-53.8$ & -2.1 \\
\hline 35 & 17 & 135028 & 1288 & 240 & 40 & 51410.4 & $51435: 3$ & -24.9 & 51419.2 & 51473.0 & $\mid-53.8$ & 4.4 \\
\hline 35 & 17 & 135036 & 1290 & 240 & 35 & 51400.0 & 51424.9 & $|-24.9|$ & 51404.0 & 51457.8 & $|-53.8|$ & 2.0 \\
\hline 35 & 17 & 135044 & 1292 & 240 & 30 & 51395.8 & 51420.8 & $|-25.0|$ & 51398.6 & 51452.4 & $\mid-53.8$ & 1.4 \\
\hline 35 & 17 & 135051 & 1294 & 240 & 25 & .51395 .2 & 51420.2 & $\mid-25.0$ & 51404.8 & 51458.6 & $\mid-53.8$ & 4.8 \\
\hline 35 & 17 & 135059 & 1296 & 240 & 20 & 51394.8 & 51419.8 & $|-25.0|$ & 51403.0 & 51456.8 & $|-53.8|$ & 4.1 \\
\hline 35 & 17 & 135106 & 1298 & 240 & 15 & 51395.2 & 51420.2 & $|-25.0|$ & 51400.2 & 51454.1 & $|-53.9|$ & 2.5 \\
\hline 35 & 17 & 135114 & 1300 & 240 & 10 & 51396.0 & 51421.1 & -25.1 & 51402.0 & 51455.9 & $|-53.9|$ & 3.0 \\
\hline 35 & 17 & 135122 & 1302 & 240 & 5 & 51395.8 & 51420.9 & -25.1 & 51405.6 & 51459.5 & $\mid-53.9$ & 4.9 \\
\hline 35 & 17 & 135130 & 1304 & 240 & 0 & 51398.6 & 51423.7 & -25.1 & 51398.0 & 51451.9 & $\mid-53.9$ & -0.3 \\
\hline 36 & 17 & 140040 & 1316 & 230 & 0 & 51405.6 & 51428.8 & $|-23.2|$ & 51413.6 & 51462.5 & $\mid-48.9$ & 4.0 \\
\hline 36 & 17 & 140048 & 1318 & 230 & 5 & 51405.2 & 51428.5 & $\mid-23.3$ & 51412.4 & 51461.4 & -49.0 & 3.6 \\
\hline 36 & 17 & 140057 & 1320 & 230 & 10 & 51408.8 & 51432.1 & $|-23.3|$ & 51406.0 & 51454.9 & $-48.9 \mid$ & -1.4 \\
\hline 36 & 17 & 140106 & 1322 & 230 & 15 & 51406.8 & 51430.1 & $|-23.3|$ & 51408.2 & 51457.0 & $|-48.8|$ & 0.7 \\
\hline 36 & 17 & 140113 & 1324 & 230 & 20 & 51403.0 & 51426.2 & $\mid-23.2$ & 51414.0 & 51462.7 & $|-48.7|$ & 5.5 \\
\hline 36 & 17 & 140121 & 1326 & 230 & 25 & 51403.6 & 51426.8 & $|-23.2|$ & 51410.0 & 51458.6 & $|-48.6|$ & 3.2 \\
\hline 36 & 17 & 140131 & 1328 & 230 & 30 & 51401.6 & 51424.8 & $|-23.2|$ & 51417.2 & 51465.7 & -48.5 & 7.8 \\
\hline 36 & 17 & 140138 & 1330 & 230 & 35 & 51396.8 & 51419.9 & $|-23.1|$ & 51402.8 & 51451.3 & -48.5 & 3.0 \\
\hline 36 & 17 & 140146 & 1332 & 230 & 40 & 51371.2 & 51394.3 & $|-23.1|$ & 51348.6 & 51397.0 & $|-48.4|$ & -11.3 \\
\hline 36 & 17 & 140154 & 1334 & 230 & 45 & 51333.2 & 51356.3 & $|-23.1|$ & 51102.0 & 51150.3 & $-48.3 \mid$ & -115.6 \\
\hline 36 & 17 & 140203 & 1336 & 230 & 50 & 51388.0 & 51411.0 & $|-23.0|$ & 51394.6 & 51442.8 & $\mid-48.2$ & 3.3 \\
\hline 36 & 17 & 140212 & 1338 & 230 & 55 & 51401.4 & 51424.4 & $|-23.0|$ & 51403.2 & 51451.3 & -48.1 & 0.9 \\
\hline 36 & 17 & 140227 & 1340 & 230 & 60 & 51403.2 & 51426.1 & $|-22.9|$ & 51403.8 & 51451.8 & -48.0 & 0.3 \\
\hline 36 & 17 & 140236 & 1342 & 230 & 65 & 51405.0 & 51427.9 & $|-22.9|$ & 51402.6 & 51450.5 & -47.9 & -1.2 \\
\hline 36 & 17 & $|140243|$ & 1344 & 230 & 70 & 51406.8 & 51429.7 & $|-22.9|$ & 51406.0 & 51453.8 & -47.8 & -0.4 \\
\hline
\end{tabular}




\begin{tabular}{|c|c|c|c|c|c|c|c|c|c|c|c|c|}
\hline Line & Day & mine & ta. & $\mathbf{X}$ & $\mathbf{Y}$ & uncorr. & $\begin{array}{l}\text { (nT) } \\
\text { corr. }\end{array}$ & aitr. & $\begin{array}{r}\text { BO7 } \\
\text { uncorr. }\end{array}$ & $\begin{array}{l}\text { TOM (nT } \\
\text { corr. }\end{array}$ & diff. & $\begin{array}{l}\text { Vert.Grad. } \\
\text { (nT/ft) }\end{array}$ \\
\hline 36 & 17 & 140252 & 1346 & 230 & 75 & 51404.4 & 51427.2 & -22.8 & 51403.0 & 51450.7 & -47.7 & -0.7 \\
\hline 36 & 17 & 140300 & 1348 & 230 & 80 & 51398.8 & 51421.6 & $\mid-22.8$ & 51391.4 & 51439.0 & -47.6 & -3.7 \\
\hline 36 & 17 & 140308 & 1350 & 230 & 85 & 51398.4 & 51421.2 & $|-22.8|$ & 51392.6 & 51440.1 & $-47.5 \mid$ & -2.9 \\
\hline 36 & 17 & 140317 & 1352 & 230 & 90 & 51400.4 & 51423.1 & $|-22.7|$ & 51385.8 & 51433.2 & $-47.4 \mid$ & -7.3 \\
\hline 36 & 17 & 140324 & 1354 & 230 & 95 & 51402.2 & 51424.9 & $\mid-22.7$ & 51392.8 & 51440.2 & -47.4 & -4.7 \\
\hline 36 & 17 & 140332 & 1356 & 230 & 100 & 51399.6 & 51422.3 & $|-22.7|$ & 51391.2 & 51438.5 & -47.3 & -4.2 \\
\hline 36 & 17 & 140340 & 1358 & 230 & 105 & 51412.8 & 51435.4 & $|-22.6|$ & 51410.0 & 51457.2 & -47.2 & -1.4 \\
\hline 36 & 17 & 140349 & 1360 & 230 & 110 & 51426.4 & 51449.0 & $\mid-22.6$ & 51398.8 & 51445.8 & -47.1 & -13.8 \\
\hline 36 & 17 & 140357 & 1362 & 230 & 115 & 51453.6 & 51476.2 & $\mid-22.6$ & 51420.4 & 51467.4 & -47.0 & -16.6 \\
\hline 36 & 17 & 140405 & 1364 & 230 & 120 & 51507.0 & 51529.5 & $|-22.5|$ & 51458.4 & 51505.3 & -46.9 & -24.3 \\
\hline 36 & 17 & 140413 & 1366 & 230 & 125 & 51617.0 & 51639.5 & -22.5 & 51529.4 & 51576.2 & -46.8 & -43.8 \\
\hline 36 & 17 & 140421 & 1368 & 230 & 130 & 51871.8 & 51894.3 & -22.5 & 51762.6 & 51809.4 & -46.8 & -54.6 \\
\hline 36 & 17 & 140431 & 1370 & 230 & 135 & 52401.2 & 52423.6 & $|-22.4|$ & 52445.2 & 52491.9 & -46.7 & 22.0 \\
\hline 36 & 17 & 140440 & 1372 & 230 & 140 & 53008.8 & 53031.2 & $\mid-22.4$ & 53542.4 & 53589.0 & -46.6 & 266.8 \\
\hline 36 & 17 & 140448 & 1374 & 230 & 145 & 53133.2 & 53155.6 & $|-22.4|$ & 53884.0 & 53930.5 & -46.5 & 375.4 \\
\hline 36 & 17 & 140456 & 1376 & 230 & 150 & 52610.0 & 52632.3 & -22.3 & 53056.8 & 53103.2 & $-46.4 \mid$ & 223.4 \\
\hline 36 & 17 & 140504 & 1378 & 230 & 155 & 51424.6 & 51446.9 & $\mid-22.3$ & 51306.4 & 51352.7 & -46.3 & -59.1 \\
\hline 36 & 17 & 140512 & 1380 & 230 & 160 & 50704.2 & 50726.5 & $\mid-22.3$ & 50251.0 & 50297.2 & -46.2 & -226.6 \\
\hline 36 & 17 & 140520 & 1382 & 230 & 165 & 50577.8 & 50600.0 & $\mid-22.2$ & 50316.0 & 50362.2 & -46.2 & -130.9 \\
\hline 36 & 17 & 140528 & 1384 & 230 & 170 & 50764.0 & 50786.2 & $\mid-22.2$ & 50585.2 & 50631.3 & -46.1 & -89.4 \\
\hline 36 & 17 & 140535 & 1386 & 230 & 175 & 51117.8 & 51140.0 & $\mid-22.2$ & 51052.4 & 51098.4 & -46.0 & -32.7 \\
\hline 36 & 17 & 140542 & 1388 & 230 & 180 & 51509.2 & 51531.3 & $|-22.1|$ & 51473.4 & 51519.3 & -45.9 & -17.9 \\
\hline 36 & 17 & 140549 & 1390 & 230 & 185 & 52039.4 & 52061.5 & $\mid-22.1$ & .52072 .0 & 52117.9 & -45.9 & 16.3 \\
\hline 36 & 17 & 140556 & 1392 & 230 & 190 & 52705.6 & 52727.7 & $|-22.1|$ & 52962.6 & 53008.4 & -45.8 & 128.5 \\
\hline 36 & 17 & 140604 & 1394 & 230 & 195 & 53377.8 & 53399.9 & $|-22.1|$ & 53873.4 & |53919.1 & -45.7 & 247.8 \\
\hline 36 & 17 & 140611 & 1396 & 230 & 200 & 53496.2 & 53518.2 & $|-22.0|$ & 54587.8 & 54633.4 & -45.6 & 545.8 \\
\hline 36 & 17 & 140619 & 1398 & 230 & 205 & 52973.0 & 52995.0 & $|-22.0|$ & 54212.8 & 54258.3 & -45.5 & 619.9 \\
\hline 36 & 17 & 140628 & 1400 & 230 & 210 & 52155.4 & 52177.4 & $|-22.0|$ & 52278.2 & 52323.7 & -45.5 & 61.4 \\
\hline 36 & 17 & 140636 & 1402 & 230 & 215 & 51345.0 & 51366.9 & $\mid-21.9$ & 51128.0 & 51173.4 & -45.4 & -108.5 \\
\hline 36 & 17 & 140643 & 1404 & 230 & 220 & 51096.0 & 51117.9 & $\mid-21.9$ & 50955.0 & 51000.3 & -45.3 & -70.5 \\
\hline 36 & 17 & 140650 & 1406 & 230 & 225 & 51090.4 & 51112.3 & $\mid-21.9$ & 51020.0 & 51065.2 & -45.2 & -35.2 \\
\hline 36 & 17 & 140658 & 1408 & 230 & 230 & 51142.0 & 51163.8 & -21.8 & $\cdot 51106.4$ & 51151.5 & -45.1 & -17.8 \\
\hline 36 & 17 & 140706 & 1410 & 230 & 235 & 51197.2 & 51219.0 & -21.8 & 51183.0 & 51228.1 & -45.1 & -7.1 \\
\hline 36 & 17 & 140718 & 1412 & 230 & 240 & 51241.0 & 51262.8 & -21.8 & 51229.6 & 1274.5 & -44.9 & -5.7 \\
\hline 36 & 17 & 140726 & 1414 & 230 & 245 & 78.2 & 51299.9 & $|-21.7|$ & 51284.2 & 329.0 & $|-44.8|$ & 3.0 \\
\hline 36 & 17 & 140734 & 1416 & 230 & 250 & 09.6 & 51331.3 & $|-21.7|$ & 51305.8 & 350.6 & -44.8 & -1.9 \\
\hline 36 & 17 & 140859 & 1418 & 230 & 255 & 36.0 & 51357.3 & $\mid-21.3$ & 51326.8 & 51370.7 & -43.9 & -4.6 \\
\hline 36 & 17 & 140907 & 1420 & 230 & 260 & 51345.2 & 51366.5 & $\mid-21.3$ & 51358.0 & 51401.8 & -43.8 & 6.4 \\
\hline 36 & 17 & 140915 & 1422 & 230 & 265 & 51363.6 & 51384.9 & $\mid-21.3$ & 51366.8 & 51410.5 & $\mid-43.7$ & 1.6 \\
\hline 36 & 17 & 140924 & 1424 & 230 & 270 & 51375.4 & 51396.6 & $\mid-21.2$ & 51377.0 & 51420.6 & -43.6 & 0.8 \\
\hline 36 & 17 & 140932 & 1426 & 230 & 275 & 51384.0 & 51405.2 & -21.2 & 51388.2 & 51431.7 & -43.5 & 2.1 \\
\hline 36 & 17 & 140940 & 1428 & 230 & 280 & 51394.0 & 51415.2 & $\mid-21.2$ & 51392.4 & 51435.9 & -43.5 & -0.8 \\
\hline 36 & 17 & 140949 & 1430 & 230 & 285 & 51401.4 & 51422.5 & $\mid-21.1$ & 51399.6 & 51443.0 & -43.4 & -0.9 \\
\hline 36 & 17 & 140956 & 1432 & 230 & 290 & 51405.0 & 51426.1 & $|-21.1|$ & 51405.2 & 51448.5 & -43.3 & 0.1 \\
\hline 36 & 17 & 141003 & 1434 & 230 & 295 & 51409.4 & 51430.5 & $\mid-21.1$ & 51411.8 & 51455.0 & -43.2 & 1.2 \\
\hline 36 & 17 & 141009 & 1436 & 230 & 300 & 51415.0 & 51436.1 & $|-21.1|$ & 51412.6 & 51455.7 & $-43.1 \mid$ & -1.2 \\
\hline 36 & 17 & 141017 & 1438 & 230 & 305 & 51417.6 & 51438.6 & $|-21.0|$ & 51416.4 & 51459.5 & $-43.1\}$ & -0.6 \\
\hline 36 & 17 & 141024 & 1440 & 230 & 310 & 51422.0 & 51443.0 & $\mid-21.0$ & 51419.4 & 51462.4 & -43.0 & -1.3 \\
\hline 36 & 17 & 141031 & 1442 & 230 & 315 & 51423.6 & 51444. & -2 & 51422.4 & 51465.3 & -42 & -0.6 \\
\hline
\end{tabular}




\begin{tabular}{|c|c|c|c|c|c|c|c|c|c|c|c|c|}
\hline Line & Day & Time & Sta. & $\mathbf{x}$ & $\mathbf{Y}$ & uncorr. & $\begin{array}{l}\text { (nT) } \\
\text { corr. }\end{array}$ & | diff. & $\begin{array}{l}\text { BOT } \\
\text { uncorr. }\end{array}$ & $\begin{array}{c}\text { TOM (nT } \\
\text { corr. }\end{array}$ & diff. & $\begin{array}{c}\text { Vert.Grad. } \\
\text { (nT/ft) }\end{array}$ \\
\hline 36 & 17 & 141039 & 1444 & 230 & 320 & 51426.8 & 51447.7 & -20.9 & 51423.2 & 51466.0 & -42.8 & -1.8 \\
\hline 37 & 17 & 141906 & 1446 & 220 & 325 & 51427.6 & 51446.5 & -18.9 & 51440.6 & 51478.2 & -37.6 & 6.5 \\
\hline 37 & 17 & 141913 & 1448 & 220 & 320 & 51427.0 & 51445.9 & -18.9 & 51431.0 & 51468.5 & -37.5 & 2.0 \\
\hline 37 & 17 & 141921 & 1450 & 220 & 315 & 51424.8 & 51443.6 & $\mid-18.8$ & 51430.8 & 51468.2 & $|-37.4|$ & 3.0 \\
\hline 37 & 17 & 141929 & 1452 & 220 & 310 & 51422.8 & 51441.6 & $|-18.8|$ & 51427.4 & 51464.7 & $|-37.3|$ & 2.3 \\
\hline 37 & 17 & 141942 & 1454 & 220 & 305 & 51419.0 & 51437.8 & $|-18.8|$ & 51426.8 & 51464.0 & $|-37.2|$ & 3.9 \\
\hline 37 & 17 & 141950 & 1456 & 220 & 300 & 51416.8 & 51435.5 & $|-18.7|$ & 51421.6 & 51458.7 & $|-37.1|$ & 2.4 \\
\hline 37 & 17 & 141957 & 1458 & 220 & 295 & 51413.2 & 51431.9 & $\mid-18.7$ & 51416.4 & 51453.4 & -37.0 & 1.6 \\
\hline 37 & 17 & 142005 & 1460 & 220 & 290 & 51409.2 & 51427.9 & -18.7 & 51411.2 & 51448.2 & -37.0 & 1.0 \\
\hline 37 & 17 & 142015 & 1462 & 220 & 285 & 51402.8 & 51421.4 & $\mid-18.6$ & 51408.4 & 51445.2 & -36.8 & 2.8 \\
\hline 37 & 17 & 142023 & 1464 & 220 & 280 & 51395.8 & 51414.4 & $\mid-18.6$ & 51409.0 & 51445.8 & -36.8 & 6.6 \\
\hline 37 & 17 & 142030 & 1466 & 220 & 275 & 51389.4 & 51408.0 & $\mid-18.6$ & 51402.2 & 51438.9 & -36.7 & 6.4 \\
\hline 37 & 17 & 142038 & 1468 & 220 & 270 & 51380.6 & 51399.1 & $\mid-18.5$ & 51389.2 & 51425.8 & -36.6 & 4.3 \\
\hline 37 & 17 & 142045 & 1470 & 220 & $265^{\circ}$ & 51368.0 & 51386.5 & -18.5 & 51383.4 & 51419.9 & -36.5 & 7.7 \\
\hline 37. & 17 & 142053 & 1472 & 220 & 260 & 59.6 & 51378.1 & -18.5 & 51359.2 & 51395.7 & -36.5 & -0.2 \\
\hline 37 & 17 & 142104 & 1474 & 220 & 255 & 51342.6 & 51361.0 & $|-18.4|$ & 51338.2 & 51374.5 & -36.3 & -2.2 \\
\hline 37 & 17 & 142133 & 1476 & 220 & 250 & 51317.0 & 51335.3 & $\mid-18.3$ & 51317.4 & 51353.4 & -36.0 & 0.2 \\
\hline 37 & 17 & 142148 & 1478 & 220 & 245 & 51294.6 & 51312.9 & $\mid-18.3$ & 51281.2 & 51317.1 & -35.9 & -6.7 \\
\hline 37 & 17 & 142158 & 1480 & 220 & 240 & 51245.8 & 51264.0 & -18.2 & 51236.0 & 51271.8 & -35.8 & -4.9 \\
\hline 37 & 17 & 142207 & 1482 & 220 & 235 & 51175.8 & 51194.0 & $\mid-18.2$ & 51160.4 & 51196.1 & -35.7 & -7.7 \\
\hline 37 & 17 & 142217 & 1484 & 220 & 230 & 51090.4 & 51108.5 & -18.1 & 51064.2 & 1099.8 & -35.6 & -13.1 \\
\hline 37 & 17 & 142227 & 1486 & 220 & 225 & 50951.8 & 50969.9 & -18.1 & 50892.8 & j928.3 & -35.5 & -29.5 \\
\hline 37 & 17 & 142236 & 1488 & 220 & -220 & 50808.4 & 50826.5 & -18.1 & 50636.8 & 50672.2 & -35.4 & -85.8 \\
\hline 37 & 17 & 142243 & 1490 & 220 & 215 & 50710.6 & 50728.6 & -18.0 & 50317.4 & 50352.7 & -35.3 & -196.6 \\
\hline 37 & 17 & 142251 & 1492 & 220 & 210 & 51077.4 & 51095.4 & -18.0 & 50749.4 & 50784.6 & -35.2 & -164.0 \\
\hline 37 & 17 & 142259 & 1494 & 220 & 205 & 52367.8 & 52385.8 & -18.0 & 52340.2 & 52375.3 & -35.1 & -13.8 \\
\hline 37 & 17 & 142306 & 1496 & 220 & 200 & 54103.6 & 54121.5 & -17.9 & 58568.4 & 3603.5 & -35.1 & 2232.4 \\
\hline 37 & 17 & 142313 & 1498 & $220 \mid$ & 195 & 57932.4 & 57950.3 & $|-17.9|$ & 56794.0 & 5829.0 & -35.0 & -569.2 \\
\hline 37 & 17 & 142321 & 1500 & 220 & 190 & 53682.2 & 53700.1 & -17.9 & 55300.2 & 5335.1 & -34.9 & 809.0 \\
\hline 37 & 17 & 142328 & 1502 & 220 & 185 & 52522.2 & 52540.0 & -17.8 & 52715.4 & 2750.2 & -34.8 & 96.6 \\
\hline 37 & 17 & 142336 & 1504 & 220 & 180 & 51745.2 & 51763.0 & $|-17.8|$ & .51738 .8 & 773.6 & -34.8 & -3.2 \\
\hline 37 & 17 & 142343 & 1506 & 220 & 175 & 51223.8 . & 51241.6 & -17.8 & 51175.2 & |209.9 & -34.7 & -24.3 \\
\hline 37 & 17 & 142350 & 1508 & 220 & 170 & 50798.6 & 50816.4 & $\mid-17.8$ & 50681.4 & 716.0 & -34.6 & -58.6 \\
\hline 37 & 17 & 142357 & 1510 & 220 & 165 & 50477.8 & 50495.5 & $|-17.7|$ & 50150.4 & 184.9 & -34.5 & -163.7 \\
\hline 37 & 17 & 142404 & 1512 & $220 \mid$ & 160 & 50424.6 & 50442.3 & -17.7 & 50045.8 & 080.3 & -34.5 & -189.4 \\
\hline 37 & 17 & 142412 & 1514 & 220 & 155 & 50798.2 & 50815.9 & $|-17.7|$ & 49772.4 & 49806.8 & -34.4 & -512.9 \\
\hline 37 & 17 & 142419 & 1516 & $220 \mid$ & 150 & 51971.0 & 51988.6 & $|-17.6|$ & 51787.2 & 51821.5 & -34.3 & -91.9 \\
\hline 37 & 17 & 142427 & 1518 & $220 \mid$ & 145 & 53043.8 & 53061.4 & $\mid-17.6$ & 53853.4 & 3887.6 & $=34.2$ & 404.8 \\
\hline 37 & 17 & 142435 & 1520 & 220 & 140 & 53257.6 & 53275.2 & -17.6 & 54269.8 & 54303.9 & -34.1 & 506.1 \\
\hline 37 & 17 & 142443 & 1522 & $220 \mid$ & 135 & 52851.6 & 52869.1 & $\mid-17.5$ & 53251.0 & 53285.1 & -34.1 & 199.7 \\
\hline 37 & 17 & 142452 & 1524 & $220 \mid$ & 130 & 52070.2 & 52087.7 & -17.5 & 51972.6 & 52006.6 & -34.0 & -48.8 \\
\hline 37 & 17 & 142501 & 1526 & $220 \mid$ & 125 & 51685.8 & 51703.3 & $\mid-17.5$ & 51589.8 & 51623.7 & -33.9 & -48.0 \\
\hline 38 & 17 & 143349 & 1538 & 210 & 125 & 51656.4 & 51671.2 & -14.8 & 51561.0 & 51593.6 & -32.6 & -47.7 \\
\hline 38 & 17 & 143358 & 1540 & 210 & 130 & 52014.4 & 52028.9 & -14.5 & 51876.8 & 51908.7 & -31.9 & -68.8 \\
\hline 38 & 17 & 143407 & 1542 & $210 \mid$ & 135 & 52624.6 & 52639.0 & $|-14.4|$ & 52847.4 & 52879.3 & -31.9 & 111.4 \\
\hline 38 & 17 & 143420 & 1544 & 210 & 140 & 52935.4 & 52949.7 & $\mid-14.3$ & 53443.4 & 53475.3 & -31.9 & 254.0 \\
\hline 38 & 17 & 143428 & 1546 & 210 & 145 & 52380.8 & 52395.1 & -14.3 & 52890.6 & 52922.4 & -31.8 & 254.9 \\
\hline 38 & 17 & 143436 & 1548 & $210 \mid$ & 150 & 51232.6 & 51246.8 & $|-14.2|$ & 50711.4 & 50743.2 & -31.8 & -260.6 \\
\hline 38 & 17 & 143444 & 1550 & |210| & 155 & 50482.0 & 50496.2 & $|-14.2|$ & 50046.6 & 50078.4 & -31.8 & -217.7 \\
\hline
\end{tabular}




\begin{tabular}{|c|c|c|c|c|c|c|c|c|c|c|c|c|}
\hline & & & & & & & (nT) & & BOT & TOM (nT & & \\
\hline Line & Day & Time & Sta. & $x$ & $\mathbf{Y}$ & uncorr. & corr. & diff. & uncorr. & corr. & diff. & 70 \\
\hline 38 & 17 & 143452 & 1552 & 210 & 160 & 50244.6 & 50258.7 & -14.1 & 49764.6 & 49796.4 & -31.8 & -240.0 \\
\hline 38 & 17 & 143500 & 1554 & 210 & 165 & 50411.6 & 50425.7 & -14.1 & 50083.0 & 50114.8 & -31.8 & -164.3 \\
\hline 38 & 17 & 143507 & 1556 & 210 & 170 & 50778.8 & 50792.8 & -14.0 & 50687.0 & 50718.7 & -31.7 & -45.9 \\
\hline 38 & 17 & 143515 & 1558 & 210 & 175 & 51119.4 & 51133.4 & -14.0 & 51017.0 & 51048.7 & -31.7 & -51.2 \\
\hline 38 & 17 & 143522 & 1560 & 210 & 180 & 51446.4 & 51460.4 & -14.0 & 51348.8 & 51380.5 & -31.7 & -48.8 \\
\hline 38 & 17 & 143530 & 1562 & 210 & 185 & 51951.8 & 51965.7 & -13.9 & 51922.8 & 51954.5 & -31.7 & -14.5 \\
\hline 38 & 17 & 143538 & 1564 & 210 & 190 & 52618.4 & 52632.3 & -13.9 & 52993.4 & 53025.1 & -31.7 & 187.5 \\
\hline 38 & 17 & 143546 & 1566 & 210 & 195 & 52832.2 & 52846.0 & $\mid-13.8$ & 53778.4 & 53810.1 & -31.7 & 473.1 \\
\hline 38 & 17 & 143554 & 1568 & 210 & 200 & 51955.4 & 51969.2 & $\mid-13.8$ & 52068.6 & 52100.2 & -31.6 & 56.6 \\
\hline 38 & 17 & 143601 & 1570 & 210 & 205 & 50680.0 & 50693.7 & $\mid-13.7$ & 49905.6 & 49937.2 & -31.6 & -387.2 \\
\hline 38 & 17 & 143609 & 1572 & 210 & 210 & 50258.0 & 50271.7 & $\mid-13.7$ & 49704.4 & 49736.0 & -31.6 & -276.8 \\
\hline 38 & 17 & 143617 & 1574 & 210 & 215 & 50409.2 & 50422.8 & $\mid-13.6$ & 50081.6 & 50113.2 & -31.6 & -163.8 \\
\hline 38 & 17 & 143624 & 1576 & 210 & 220 & 50696.4 & 50710.0 & |-13.6 & 50561.8 & 50593.4 & -31.6 & -67.3 \\
\hline 38 & 17 & 143631 & .1578 & 210 & 225 & 50939.6 & 50953.1 & -13.5 & 50884.2 & 50915.8 & -31.6 & -27.7 \\
\hline 38 & 17 & 143637 & 1580 & 210 & 230 & 51073.2 & 51086.7 & -13.5 & 51060.0 & 51091.5 & -31.5 & -6.6 \\
\hline 38 & 17 & 143647 & 1582 & 210 & 235 & 51179.8 & 51193.2 & $\mid-13.4$ & 51173.2 & 51204.7 & $\mid-31.5$ & -3.3 \\
\hline 38 & 17 & 143656 & 1584 & 210 & 240 & 51248.2 & 51261.6 & $\mid-13.4$ & 51250.2 & 51281.7 & -31.5 & 1.0 \\
\hline 38 & 17 & 143708 & 1586 & 210 & 245 & 51289.4 & 51302.7 & $\mid-13.3$ & 51287.4 & 51318.9 & -31.5 & -1.0 \\
\hline 38 & 17 & 143721 & 1588 & 210 & 250 & 51321.6 & 51334.8 & $\mid-13.2$ & 51322.2 & 51353.6 & -31.4 & 0.3 \\
\hline 38 & 17 & 143733 & 1590 & 210 & 255 & 51343.8 & 51357.0 & $\mid-13.2$ & 51344.2 & 51375.6 & $|-31.4|$ & 0.2 \\
\hline 38 & 17 & 143747 & 1592 & 210 & $260^{\circ}$ & 51367.0 & 51380.1 & $\mid-13.1$ & 51365.6 & 51397.0 & $|-31.4|$ & -0.7 \\
\hline 38 & 17 & 143755 & 1594 & 210 & 265 & 51373.4 & 51386.4 & -13.0 & 51381.8 & 51413.2 & -31.4 & 4.2 \\
\hline 38 & 17 & 143803 & 1596 & 210 & 270 & 51388.8 & 51401.8 & -13.0 & 51389.6 & 51421.0 & -31.4 & 0.4 \\
\hline 38 & 17 & 143811 & 1598 & 210 & 275 & 51396.4 & 51409.3 & $\mid-12.9$ & 51398.6 & 51429.9 & -31.3 & 1.1 \\
\hline 38 & 17 & 143819 & 1600 & 210 & 280 & 51407.4 & 51420.3 & $\mid-12.9$ & 51405.4 & 51436.7 & $\mid-31.3$ & -1.0 \\
\hline 38 & 17 & 143826 & 1602 & 210 & 285 & 51412.2 & 51425.1 & $\mid-12.9$ & 51411.4 & 51442.7 & $|-31.3|$ & -0.4 \\
\hline 38 & 17 & 143834 & 1604 & 210 & 290 & 51417.8 & 51430.6 & $\mid-12.8$ & 51418.0 & 51449.3 & -31.3 & 0.1 \\
\hline 38 & 17 & 143841 & 1606 & 210 & 295 & 51422.0 & 51434.8 & -12.8 & 51419.0 & 51450.3 & -31.3 & -1.5 \\
\hline 38 & 17 & 143849 & 1608 & 210 & 300 & 51425.2 & 51437.9 & -12.7 & 51425.0 & 51456.3 & -31.3 & -0.1 \\
\hline 38 & 17 & 143856 & 1610 & 210 & 305 & 51429.6 & 51442.3 & $\mid-12.7$ & 51426.6 & 51457.8 & -31.2 & -1.5 \\
\hline 38 & 17 & 143903 & 1612 & 210 & 310 & 51430.0 & 51442.6 & $\mid-12.6$ & 51430.2 & 51461.4 & $\mid-31.2$ & 0.1 \\
\hline 38 & 17 & 143911 & 1614 & 210 & 315 & 51435.4 & 51448.0 & $\mid-12.6$ & 51432.8 & 51464.0 & $\mid-31.2$ & -1.3 \\
\hline 38 & 17 & 143919 & 1616 & 210 & 320 & 51437.0 & 51449.5 & -12.5 & 51436.0 & 51467.2 & -31.2 & -0.5 \\
\hline 38 & 17 & 143927 & 1618 & 210 & 325 & 51436.6 & 51449.1 & $\mid-12.5$ & 51434.8 & 51466.0 & -31.2 & -0.9 \\
\hline 39 & 17 & 144401 & 1620 & 200 & 305 & 51428.8 & 51439.7 & -10.9 & 51438.4 & 51468.9 & -30.5 & 4.8 \\
\hline 39 & 17 & 144409 & 1622 & 200 & 300 & 51424.8 & 51435.6 & $\mid-10.8$ & 51441.0 & 51471.5 & -30.5 & 8.1 \\
\hline 39 & 17 & 144417 & 1624 & 200 & 295 & 51423.6 & 51434.4 & $\mid-10.8$ & 51427.6 & 51458.1 & -30.5 & 2.0 \\
\hline 39 & 17 & 144425 & 1626 & 200 & 290 & 51416.6 & 51427.3 & $\mid-10.7$ & 51425.0 & 51455.5 & -30.5 & 4.2 \\
\hline 39 & 17 & 144432 & 1628 & 200 & 285 & - 51410.0 & 51420.7 & -10.7 & 51417.4 & 51447.9 & -30.5 & 3.7 \\
\hline 39 & 17 & 144439 & 1630 & 200 & 280 & 51406.2 & 51416.8 & $\mid-10.6$ & 51410.6 & 51441.1 & -30.5 & 2.2 \\
\hline 39 & 17 & 144447 & 1632 & 200 & 275 & 51398.2 & 51408.8 & $\mid-10.6$ & 51407.0 & 51437.4 & -30.4 & 4.4 \\
\hline 39 & 17 & 144455 & 1634 & 200 & 270 & 51392.4 & 51402.9 & $\mid-10.5$ & 51399.0 & 51429.4 & $|-30.4|$ & 3.3 \\
\hline 39 & 17 & 144505 & 1636 & 200 & 265 & 51379.4 & 51389.9 & -10.5 & 51394.2 & 51424.6 & -30.4 & 7.4 \\
\hline 39 & 17 & 144513 & 1638 & 200 & 260 & 51365.0 & 51375.4 & $\mid-10.4$ & 51370.0 & 51400.4 & -30.4 & 2.5 \\
\hline 39 & 17 & 144523 & 1640 & 200 & 255 & 51353.8 & 51364.2 & -10.4 & 51348.2 & 51378.6 & -30.4 & -2.8 \\
\hline 39 & 17 & 144531 & 1642 & 200 & 250 & 51321.2 & 51331.5 & -10.3 & $51332: 8$ & 51363.1 & -30.3 & 5.8 \\
\hline 39 & 17 & 144540 & 1644 & 200 & 245 & 51291.6 & 51301.9 & -10.3 & 51304.8 & 51335.1 & -30.3 & 6.6 \\
\hline 39 & 17 & 144549 & 1646 & 200 & 240 & 51255.6 & 51265.8 & -10.2 & 51264.0 & 51294.3 & -30.3 & 4.2 \\
\hline 39 & 17 & 144601 & 1648 & 200 & 235 & 51191.8 & 51201.9 & $\mid-10.1$ & 51201.2 & 51231.5 & -30.3 & 4.7 \\
\hline
\end{tabular}




\begin{tabular}{|c|c|c|c|c|c|c|c|c|c|c|c|c|}
\hline & & & & & & & (nT) & & BOT & TOM (nT) & & Vert.Grad. \\
\hline Line & Day & Time & Sta. & $\mathbf{X}$ & $\mathbf{Y}$ & uncorr. & corr. & diff. & uncorr. & corr. & diff. & \\
\hline 39 & 17 & 144609 & 1650 & 200 & 230 & 51117.8 & 51127.9 & -10.1 & 51124.0 & 51154.3 & -30.3 & 3.1 \\
\hline 39 & 17 & 144616 & 1652 & 200 & 225 & 51002.0 & 51012.0 & -10.0 & 50985.4 & 51015.6 & -30.2 & -8.3 \\
\hline 39 & 17 & 144624 & 1654 & 200 & 220 & 50849.6 & 50859.6 & $\mid-10.0$ & 50724.0 & 50754.2 & -30.2 & -62.8 \\
\hline 39 & 17: & 144634 & 1656 & 200 & 215 & 51093.4 & 51103.3 & -9.9 & 53684.6 & 53714.8 & -30.2 & 1295.6 \\
\hline 39 & 17 & 144641 & 1658 & 200 & 210 & 50722.2 & 50732.1 & -9.9 & 50398.6 & 50428.8 & -30.2 & -161.8 \\
\hline 39 & 17 & 144650 & 1660 & 200 & 205 & 51056.6 & 51066.4 & -9.8 & 50966.6 & 50996.8 & $|-30.2|$ & -45.0 \\
\hline 39 & 17 & 144657 & 1662 & 200 & 200 & 51437.2 & 51447.0 & -9.8 & 51281.6 & 51311.8 & -30.2 & -77.8 \\
\hline 39 & 17 & 144705 & 1664 & 200 & 195 & 51745.6 & 51755.4 & -9.8 & 51811.2 & 51841.3 & $|-30.1|$ & 32.8 \\
\hline 39 & 17 & 144712 & 1666 & 200 & 190 & 51703.8 & 51713.5 & -9.7 & 51730.6 & 51760.7 & $\mid-30.1$ & 13.4 \\
\hline 39 & 17 & 144720 & 1668 & 200 & 185 & 51485.6 & 51495.3 & -9.7 & 51440.6 & 51470.7 & $\mid-30.1$ & -22.5 \\
\hline 39 & 17 & 144727 & 1670 & 200 & 180 & 51266.4 & $\mid 51276.0$ & -9.6 & 51190.4 & 51220.5 & $|-30.1|$ & -38.0 \\
\hline 39 & 17 & 144735 & 1672 & 200 & 175 & 51026.4 & 51036.0 & -9.6 & 50971.2 & 51001.3 & $|-30.1|$ & -27.6 \\
\hline 39 & 17 & 144743 & 1674 & 200 & 170 & 50747.8 & 50757.3 & -9.5 & 50623.0 & 50653.0 & $|-30.0|$ & -62.4 \\
\hline 39 & 17 & 144751 & 1676 & 200 & 165 & 50404.4 & $50413: 9$ & -9.5 & 49810.8 & 49840.8 & -30.0 & -296.8 \\
\hline 39 & 17 & 144759 & 1678 & 200 & 160 & 50522.6 & 50532.0 & -9.4 & 50520.8 & 50550.8 & -30.0 & -0.9 \\
\hline 39 & 17 & 144807 & 1680 & 200 & 155 & 50533.6 & 50543.0 & -9.4 & 49942.4 & 49972.4 & -30.0 & -295.6 \\
\hline 39 & 17 & 144815 & 1682 & 200 & 150 & 51129.8 & 51139.1 & -9.3 & 50486.0 & 50516.0 & -30.0 & -321.9 \\
\hline 39 & 17 & 144823 & 1684 & 200 & 145 & 51.8 & 52361.1 & -9.3 & 52653.2 & 52683.2 & $|-30.0|$ & 150.7 \\
\hline 39 & 17 & 144831 & 1686 & 200 & 140 & 52980.2 & 52989.4 & -9.2 & 54123.4 & 54153.3 & $|-29.9|$ & 571.6 \\
\hline 39 & 17 & 144840 & 1688 & 200 & 135 & 52651.8 & $|52661.0|$ & -9.2 & 53014.0 & 53043.9 & $|-29.9|$ & 181.1 \\
\hline 39 & 17 & 144849 & 1690 & 200 & 130 & 51964.8 & 51973.9 & -9.1 & 51860.2 & 51890.1 & $|-29.9|$ & -52.3 \\
\hline 39 & 17 & 144857 & 1692 & 200 & 125 & 51639.0 & 51648.1 & -9.1 & 51527.0 & 51556.9 & -29.9 & -56.0 \\
\hline 40 & 18 & 111453 & 10 & 190 & 139.37 & 52868.2 & 52874.8 & -6.6 & 54044.2 & 54080.0 & -35.8 & 588.0 \\
\hline 40 & 18 & 111503 & 12 & 190 & 144.37 & 52318.0 & 52324.6 & -6.6 & 52580.0 & 52615.6 & $|-35.6|$ & 131.0 \\
\hline 40 & 18 & 111512 & 14 & 190 & 149.37 & 51497.2 & 51503.7 & -6.5 & 51281.6 & 51317.1 & $-35.5 \mid$ & -107.8 \\
\hline 40 & 18 & 111521 & 16 & 190 & 154.37 & 50924.4 & 50930.9 & -6.5 & $50644.0^{\circ}$ & 50679.4 & $|-35.4|$ & -140.2 \\
\hline 40 & 18 & 111529 & 18 & 190 & 159.37 & 50670.0 & 50676.4 & -6.4 & 50319.4 & 50354.7 & -35.3 & -175.3 \\
\hline 40 & 18 & 111537 & 20 & 190 & 164.37 & 50649.2 & 50655.6 & -6.4 & 50369.6 & 0404.8 & -35.2 & -139.8 \\
\hline 40 & 18 & 111544 & 22 & 190 & 169.37 & 50878.0 & 50884.3 & -6.3 & 50778.0 & 50813.0 & -35.0 & -50.0 \\
\hline 40 & 18 & 111552 & 24 & 190 & 174.37 & 51177.8 & 51184.1 & -6.3 & 51122.0 & 1156.9 & -34.9 & -27.9 \\
\hline 40 & 18 & 111559 & 26 & 190 & 179.37 & 51461.0 & 51467.2 & -6.2 & 51427.6 & 51462.4 & -34.8 & -16.7 \\
\hline 40 & 18 & 111606 & 28 & 190 & 184.37 & 51826.4 & 51832.6 & -6.2 & 51871.8 & 51906.5 & -34.7 & 22.7 \\
\hline 40 & 18 & 111614 & 30 & 190 & 189.37 & 52359.0 & 52365.1 & -6.1 & 52726.8 & 52761.4 & $|-34.6|$ & 183.9 \\
\hline 40 & 18 & 111622 & 32 & 190 & 194.37 & 52566.6 & 52572.7 & -6.1 & 53667.6 & 53702.1 & $|-34.5|$ & 550.5 \\
\hline 40 & 18 & 111630 & 34 & 190 & 199.37 & 52286.2 & 52292.2 & -6.0 & 52955.0 & 52989.3 & -34.3 & 334.4 \\
\hline 40 & 18 & 111638 & 36 & 190 & 204.37 & 51426.4 & 51432.4 & -6.0 & 51354.2 & 51388.4 & -34.2 & -36.1 \\
\hline 40 & 18 & 111646 & 38 & 190 & 209.37 & 50825.8 & $|50831.7|$ & -5.9 & 50462.4 & 50496.5 & -34.1 & -181.7 \\
\hline 40 & 18 & 111654 & 40 & 190 & 214.37 & 50744.2 & 50750.1 & -5.9 & 50528.8 & 50562.8 & -34.0 & -107.7 \\
\hline 40 & 18 & 111702 & 42 & 190 & 219.37 & 50886.4 & 50892.2 & -5.8 & 50803.8 & 50837.7 & -33.9 & -41.3 \\
\hline 40 & 18 & 111709 & 44 & 190 & 224.37 & 51027.6 & 51033.4 & -5.8 & 50999.6 & 51033.4 & -33.8 & -14.0 \\
\hline 40 & 18 & 111716 & 46 & 190 & 229.37 & 51130.4 & 51136.1 & -5.7 & 51126.2 & 51159.9 & $-33.7 \mid$ & -2.1 \\
\hline 40 & 18 & 111723 & 48 & 190 & 234.37 & 51200.0 & |51205.7 & -5.7 & 51208.6 & 51242.2 & $|-33.6|$ & 4.3 \\
\hline 40 & 18 & 111733 & 50 & 190 & 239.37 & 51250.8 & 51256.4 & -5.6 & 51263.6 & 51297.0 & -33.4 & 6.4 \\
\hline 40 & 18 & 111742 & 52 & 190 & 244.37 & 51287.2 & 51292.8 & -5.6 & 51304.8 & 51338.1 & $|-33.3|$ & 8.8 \\
\hline 40 & 18 & 111750 & 54 & 190 & 249.37 & 51317.2 & $|51322.7|$ & -5.5 & 51328.4 & 51361.6 & -33.2 & 5.6 \\
\hline 40 & 18 & 1117.58 & 56 & 190 & 254.37 & 51347.6 & 51353.1 & -5.5 & 51348.8 & 51381.8 & $|-33.0|$ & 0.6 \\
\hline 40 & 18 & 111804 & 58 & 190 & 259.31 & 51369.2 & 51374.6 & -5.4 & 51364.4 & 51397.3 & -32.9 & -2.4 \\
\hline 40 & 18 & 111813 & 60 & 190 & 264.37 & 51374.8 & 51380.2 & -5.4 & 51387.2 & 51420.0 & -32.8 & 6.2 \\
\hline r & 18 & $1110<2]$ & $0<$ & 190 & 269.37 & 51386.4 & $|51391.7|$ & -0.0 & 51395.4 & 51428.1 & $|-32.7|$ & 4.5 \\
\hline
\end{tabular}




\begin{tabular}{|c|c|c|c|c|c|c|c|c|c|c|c|c|}
\hline & & & & & & & & & & & & \\
\hline ne & Day & Time & Sta. & $\mathbf{x}$ & $\mathbf{Y}$ & uncort. & corr. & diff. & corr. & & dit & \\
\hline 40 & 18 & 111831 & 64 & 190 & 274.37 & 51399.4 & 51404.7 & -5.3 & 51403.2 & 51435.8 & -32.6 & 1.9 \\
\hline 40 & 18 & 111838 & 66 & 190 & 279.37 & 51408.4 & 51413.6 & -5.2 & 51416.2 & 51448.6 & -32.4 & 3.9 \\
\hline 40 & 18 & 111846 & 68 & 190 & 284.37 & 51414.4 & $\mid 51419.6$ & -5.2 & 51413.2 & 51445.5 & $\mid-32.3$ & -0.6 \\
\hline 40 & 18 & 111853 & -70 & 190 & 289.37 & 51418.8 & 51423.9 & -5.1 & 51418.4 & 51450.6 & $\mid-32.2$ & -0.2 \\
\hline 40 & 18 & 111901 & 72 & 190 & 294.37 & 51424.0 & 51429.1 & -5.1 & 51423.4 & 51455.5 & -32.1 & -0.3 \\
\hline 40 & 18 & 111908 & 74 & 190 & 299.37 & 51430.0 & 51435.1 & -5.1 & 51428.6 & 51460.6 & -32.0 & -0.7 \\
\hline 40 & 18 & 111915 & 76 & 190 & 304.37 & 51433.8 & 51438.8 & -5.0 & 51445.2 & 51477.1 & -31.9 & 5.7 \\
\hline 40 & 18 & 111922 & 78 & 190 & 309.37 & 51429.2 & 51434.2 & -5.0 & 51417.8 & 51449.6 & $\mid-31.8$ & -5.7 \\
\hline 40 & 18 & 111930 & 80 & 190 & 314.37 & 51432.0 & 51436.9 & -4.9 & 51434.6 & 51466.3 & $\mid-31.7$ & $1: 3$ \\
\hline 40 & 18 & 111937 & 82 & 190 & 319.37 & 51437.6 & 51442.5 & -4.9 & 51436.6 & 51468.2 & -31.6 & -0.5 \\
\hline 41 & 18 & 112231 & 84 & 180 & 318.74 & 51433.2 & 51437.0 & -3.8 & 51443.0 & 51471.9 & -28.9 & 4.9 \\
\hline 41 & 18 & 112238 & 86 & 180 & 313.74 & 51431.8 & 51435.5 & -3.7 & 51442.0 & 51470.8 & -28.8 & 5.1 \\
\hline 41 & 18 & 112246 & 88 & 180 & 308.74 & 51430.4 & 51434.1 & -3.7 & 51442.4 & 51471.1 & -28.7 & 6.0 \\
\hline 41 & 18 & 112253 & 90 & 180 & 303.74 & 51427.2 & 51430.9 & -3.7 & 51435.0 & 51463.6 & -28.6 & 3.9 \\
\hline 41 & 18 & 112301 & 92 & 180 & 298.74 & 51422.4 & 51426.0 & -3.6 & 51433.6 & 51462.1 & -28.5 & 5.6 \\
\hline 41 & 18 & 112308 & 94 & 180 & 293.74 & 51417.0 & 51420.6 & -3.6 & 51429.6 & 51458.0 & $\mid-28.4$ & 6.3 \\
\hline 41 & 18 & 112315 & 96 & 180 & 288.74 & 51411.4 & 51414.9 & -3.5 & 51417.8 & 51446.1 & -28.3 & 3.2 \\
\hline 41 & 18 & 112322 & 98 & 180 & 283.74 & 51408.0 & 51411.5 & -3.5 & $51415 . \dot{8}$ & 51444.0 & -28.2 & 3.9 \\
\hline 41 & 18 & 112329 & 100 & 180 & 278.74 & 51402.8 & 51406.2 & -3.4 & 51416.6 & 51444.7 & $\mid-28.1$ & 6.9 \\
\hline 41 & 18 & 112337 & 102 & 180 & 273.74 & 51395.0 & 51398.4 & -3.4 & 51403.2 & 51431.2 & -28.0 & 4.1 \\
\hline 41 & 18 & 112346 & 104 & 180 & 268.74 & 51384.0 & 51387.3 & -3.3 & 51397.8 & 51425.6 & -27.8 & 6.9 \\
\hline 41 & 18 & 112353 & 106 & 180 & 263.74 & 51368.2 & 51371.5 & -3.3 & 51371.4 & 51399.1 & -27.7 & 1.6 \\
\hline 41 & 18 & 112400 & 108 & 180 & 258.74 & 51355.0 & 358.2 & -3.2 & 51365.6 & 393.2 & $\mid-27.6$ & 5.3 \\
\hline 41 & 18 & 112407 & 110 & 180 & 253.74 & 51332.6 & 335.8 & -3.2 & 51329.4 & 356.9 & -27.5 & -1.6 \\
\hline 41 & 18 & 112417 & 112 & 180 & 248.74 & 51311.8 & 51314.9 & -3.1 & 51308.0 & 51335.4 & $\mid-27.4$ & -1.9 \\
\hline 41 & 18 & 112425 & 114 & 180 & 243.74 & 51276.0 & 51279.1 & -3.1 & 51281.6 & 51308.8 & -27.2 & 2.8 \\
\hline 41 & 18 & 112433 & 116 & 180 & 238.74 & 51240.6 & 51243.6 & $: 3.0$ & 51248.2 & 51275.3 & -27.1 & 3.8 \\
\hline 41 & 18 & 112440 & 118 & 180 & 233.74 & 91.0 & 51194.0 & -3.0 & 51201.4 & 51228.4 & -27.0 & 5.2 \\
\hline 41 & 18 & 112447 & 120 & 180 & 228.74 & 27.6 & 51130.5 & -2.9 & 51123.8 & 51150.7 & $\mid-26.9$ & -1.9 \\
\hline 41 & 18 & 112455 & 122 & 180 & 223.74 & 51022.0 & 51024.9 & -2.9 & 50993.6 & 51020.4 & -26.8 & -14.2 \\
\hline 41 & 18 & 112502 & 124 & 180 & 218.74 & 50893.8 & 50896.7 & -2.9 & 50803.4 & 50830.1 & -26.7 & -45.2 \\
\hline 41 & 18 & 112510 & 126 & 180 & 213.74 & 50761.2 & 50764.0 & -2.8 & 50538.8 & 50565.4 & $\mid-26.6$ & -111.2 \\
\hline 41 & 18 & 112517 & 128 & 180 & 208.74 & 50742.6 & 50745.4 & -2.8 & 50345.4 & 50371.9 & -26.5 & -198.6 \\
\hline 41 & 18 & 112525 & 130 & 180 & 203.74 & 50987.0 & 50989.7 & -2.7 & 50311.0 & 50337.4 & $\mid-26.4$ & -338.0 \\
\hline 41 & 18 & 112533 & 132 & 180 & 198.74 & 51633.0 & 51635.7 & -2.7 & 51384.0 & 51410.2 & -26.2 & -124.5 \\
\hline 41 & 18. & 112540 & 134 & 180 & 193.74 & 01.4 & 52304.0 & -2.6 & 52642.6 & 52668.7 & -26.1 & 170.6 \\
\hline 41 & 18 & 112547 & 136 & 180 & 188.74 & 52309.4 & 52312.0 & -2.6 & 52694.8 & 52720.8 & -26.0 & 192.7 \\
\hline 41 & 18 & 112554 & 138 & 180 & 183.74 & 51932.0 & 51934.5 & -2.5 & 52024.2 & 52050.1 & -25.9 & 46.1 \\
\hline 41 & 18 & 112601 & 140 & 180 & 178.74 & 51561.0 & 51563.5 & -2.5 & 51559.8 & 51585.6 & $\mid-25.8$ & -0.6 \\
\hline 41 & 18 & 112608 & 142 & 180 & 173.74 & '51237.6 & 51240.0 & -2.4 & 51207.0 & 51232.7 & $\mid-25.7$ & -15.3 \\
\hline 41 & 18 & 112615 & 144 & 180 & 168.74 & 50894.8 & 50897.2 & -2.4 & 50764.6 & 50790.2 & $\mid-25.6$ & -65.1 \\
\hline 41 & 18 & 112622 & 146 & 180 & 163.74 & 50685.8 & 50688.2 & -2.4 & 50342.6 & 50368.1 & $\mid-25.5$ & -171.6 \\
\hline 41 & 18 & 112630 & 148 & 180 & 158.74 & 50812.8 & 50815.1 & -2.3 & 50704.6 & 50730.0 & $\mid-25.4$ & -54.1 \\
\hline 41 & 18 & 112638 & 150 & 180 & 153.74 & 163.6 & 51165.9 & -2.3 & 51250.6 & 51275.9 & -25.3 & 43.5 \\
\hline 41 & 18 & 112645 & 152 & 180 & 148.74 & 519.6 & 51521.8 & -2.2 & 51198.4 & 51223.6 & $\mid-25.2$ & -160.6 \\
\hline 41 & 18 & 112654 & 154 & 180 & 143.74 & 52285.8 & 52288.0 & -2.2 & 52262.2 & 52287.2 & -25.0 & -11.8 \\
\hline 41 & 18 & 112702 & 156 & 180 & 138.74 & 52727.0 & 52729.1 & -2.1 & 53931.2 & 53956.1 & -24.9 & 602.1 \\
\hline 42 & 18 & 113314 & 168 & 170 & 123.11 & 51464.0 & 51469.4 & -5.4 & 51353.0 & 51377.2 & -24.2 & -55.5 \\
\hline 42 & 18 & 113323 & 170 & 170 & 128.11 & 51745.2 & 51750.5 & -5.3 & 51566.0 & 51589.9 & -23.9 & -89.6 \\
\hline
\end{tabular}




\begin{tabular}{|c|c|c|c|c|c|c|c|c|c|c|c|c|}
\hline & & & & & & & (nT) & & BO7 & TOM (nT) & & Vert.Grad. \\
\hline Line & Day & Time & Sta. & $\mathbf{X}$ & $\mathbf{Y}$ & uncorr. & corr. & diff. & uncorr. & corr. & diff. & \\
\hline 42 & 18 & 113332 & 172 & 170 & 133.11 & 52508.4 & 52513.8 & -5.4 & 52843.8 & 52867.6 & -23.8 & 167.7 \\
\hline 42 & 18 & 113340 & 174 & 170 & 138.11 & 53152.0 & 53157.5 & -5.5 & 54109.6 & 54133.4 & -23.8 & 478.8 \\
\hline 42 & 18 & 113348 & 176 & 170 & 143.11 & 52541.6 & 52547.2 & -5.6 & 55646.6 & 55670.4 & -23.8 & 1552.5 \\
\hline 42 & 18 & 113356 & 178 & 170 & 148.11 & 51437.2 & 51442.9 & -5.7 & 51414.6 & 51438.4 & -23.8 & -11.3 \\
\hline 42 & 18 & 113403 & 180 & 170 & 153.11 & 50665.2 & 50670.9 & -5.7 & 50116.8 & $\mid 50140.6$ & $\mid-23.8$ & -274.2 \\
\hline 42 & 18 & 113411 & 182 & 170 & 158.11 & 50343.4 & 50349.2 & -5.8 & 49815.0 & 49838.8 & -23.8 & -264.2 \\
\hline 42 & 18 & 113418 & 184 & 170 & 163.11 & 50495.8 & 50501.7 & -5.9 & 50116.8 & 50140.6 & $\mid-23.8$ & -189.5 \\
\hline 42 & 18 & 113425 & 186 & 170 & 168.11 & 50911.6 & 50917.6 & -6.0 & 50797.2 & 50821.0 & -23.8 & -57.2 \\
\hline 42 & 18 & 113432 & 188 & 170 & 173.11 & 51330.8 & 51336.8 & -6.0 & 51265.2 & 51288.9 & -23.7 & -32.8 \\
\hline 42 & 18 & 113439 & 190 & 170 & 178.11 & 51710.6 & 51716.7 & -6.1 & 51698.2 & 51721.9 & -23.7 & -6.2 \\
\hline 42 & 18 & 113446 & 192 & 170 & 183.11 & 52130.0 & 52136.2 & -6.2 & 52140.6 & 52164.3 & $\mid-23.7$ & 5.3 \\
\hline 42 & 18 & 113452 & 194 & 170 & 188.11 & 52487.2 & 52493.4 & -6.2 & 52573.4 & 52597.1 & $\mid-23.7$ & 43.1 \\
\hline 42 & 18 & 113459 & 196 & 170 & 193.11 & 52567.4 & 52573.7 & -6.3 & 52749.8 & 52773.5 & -23.7 & 91.2 \\
\hline 42 & 18 & 113507 & 198 & 170 & 198.11 & 52218.4 & 52224.8 & -6.4 & 52253.2 & |52276.9 & -23.7 & 17.4 \\
\hline 42 & 18 & 113514 & 200 & 170 & 203.11 & 51627.2 & 51633.6 & -6.4 & 51489.8 & 51513.5 & -23.7 & -68.7 \\
\hline 42 & 18 & 113521 & 202 & 170 & 208.11 & 51178.4 & 51184.9 & -6.5 & 50947.6 & 50971.3 & $\mid-23.7$ & -115.4 \\
\hline 42 & 18 & 113528 & 204 & 170 & 213.11 & 50966.6 & 50973.2 & -6.6 & 50784.0 & 50807.7 & -23.7 & -91.3 \\
\hline 42 & 18 & 113535 & 206 & 170 & 218.11 & 50960.6 & 50967.3 & -6.7 & 50858.0 & 50881.6 & -23.6 & -51.3 \\
\hline 42 & 18 & 113545 & 208 & 170 & 223.11 & 51027.2 & 51034.0 & -6.8 & 50991.4 & 51015.0 & -23.6 & $-17.9^{\circ}$ \\
\hline 42 & 18 & 113553 & 210 & 170 & 228.11 & 51109.4 & 51116.2 & -6.8 & 51082.8 & 51106.4 & $\mid-23.6$ & -13.3 \\
\hline 42 & 18 & 113604 & 212 & 170 & 233.11 & 51176.2 & 51183.1 & -6.9 & 51157.4 & 51181.0 & $\mid-23.6$ & -9.4 \\
\hline 42 & 18 & 113611 & 214 & 170 & 238.11 & 51226.0 & 51233.0 & -7.0 & 51233.4 & $|51257.0|$ & $\mid-23.6$ & 3.7 \\
\hline 42 & 18 & 113618 & 216 & .170 & 243.11 & 51268.8 & 51275.9 & -7.1 & 51273.0 & 51296.6 & |-23.6 & $2.1^{\circ}$ \\
\hline 42 & 18 & 113626 & 218 & 170 & 248.11 & 51305.4 & 51312.6 & -7.2 & 51311.4 & 51335.0 & $\mid-23.6$ & 3.0 \\
\hline 42 & 18 & 113633 & 220 & 170 & 253.11 & 51333.2 & 51340.4 & -7.2 & 51338.4 & 51361.9 & -23.5 & 2.6 \\
\hline 42 & 18 & 113641 & 222 & 170 & 258.11 & 51355.6 & 51362.9 & -7.3 & 51361.2 & 1384.7 & $\mid-23.5$ & 2.8 \\
\hline 42 & 18 & 113648 & $224^{\prime}$ & 170 & 263.11 & 51366.8 & 51374.2 & -7.4 & 51373.0 & 51396.5 & -23.5 & 3.1 \\
\hline 42 & 18 & 113656 & 226 & 170 & 268.11 & 51383.2 & 51390.7 & $=7.5$ & 51376.4 & 51399.9 & $\mid-23.5$ & -3.4 \\
\hline 42 & 18 & 113704 & 228 & 170 & 273.11 & 51399.6 & 51407.1 & -7.5 & 51412.8 & 51436.3 & $|-23.5|$ & 6.6 \\
\hline 42 & 18 & 113712 & 230 & 170 & 278.11 & 51403.0 & 51410.6 & -7.6 & 51404.2 & 51427.7 & $|-23.5|$ & 0.6 \\
\hline 42 & 18 & 113720 & 232 & 170 & 283.11 & 51410.0 & 51417.7 & -7.7 & 51407.4 & 51430.9 & -23.5 & -1.3 \\
\hline 42 & 18 & 113727 & 234 & 170 & 288.11 & 51418.4 & 51426.2 & -7.8 & 51420.2 & 51443.7 & -23.5 & .0 .9 \\
\hline 42 & 18 & 113734 & 236 & 170 & 293.11 & 51422.0 & 51429.8 & -7.8 & 51419.4 & 51442.9 & -23.5 & -1.3 \\
\hline 42 & 18 & 113742 & 238 & 170 & 298.11 & 51425.2 & 51433.1 & -7.9 & 51427.4 & 51450.8 & $\mid-23.4$ & 1.1 \\
\hline 42 & 18 & 113755 & 240 & 170 & 303.11 & 51432.4 & 51440.4 & -8.0 & 51429.4 & 51452.8 & $|-23.4|$ & -1.5 \\
\hline 42 & 18 & 113802 & 242 & 170 & 308.11 & 51436.2 & 51444.3 & -8.1 & 51432.8 & 51456.2 & $\mid-23.4$ & -1.7 \\
\hline 42 & 18 & 113810 & 244 & 170 & 313.11 & 51434.8 & 51443.0 & -8.2 & 51439.2 & $\mid 51462.6$ & $\mid-23.4$ & 2.2 \\
\hline 43 & 18 & 114210 & 246 & 160 & 312.48 & 51432.6 & 51443.2 & $\mid-10.6$ & 51445.8 & 51468.8 & -23.0 & 6.6 \\
\hline 43 & 18 & 114217 & 248 & 160 & 307.48 & 51427.8 & 51438.4 & -10.6 & 51438.2 & 51461.2 & -23.0 & $5.2^{\prime}$ \\
\hline 43 & 18 & 114225 & 250 & 160 & 302.48 & 51427.0 & 51437.7 & -10.7 & 51431.0 & 454.0 & $\mid-23.0$ & 2.0 \\
\hline 43 & 18 & 114232 & 252 & 160 & 297.48 & 51422.0 & 51432.8 & -10.8 & 51432.4 & 51455.4 & -23.0 & 5.2 \\
\hline 43 & 18 & 114239 & 254 & 160 & 292.48 & 51417.4 & 51428.2 & -10.8 & 51425.4 & 51448.4 & -23.0 & 4.0 \\
\hline 43 & 18 & 114246 & 256 & 160 & 287.48 & 51412.2 & 51423.1 & $\mid-10.9$ & 51417.6 & $|51440.6|$ & -23.0 & 2.7 \\
\hline 43 & 18 & 114254 & 258 & 160 & 282.48 & 51403.8 & 51414.8 & $\mid-11.0$ & 51399.8 & 51422.7 & $\mid-22.9$ & -2.0 \\
\hline 43 & 18 & 114301 & 260 & 160 & 277.48 & 51397.8 & 51408.9 & $\mid-11.1$ & 51405.6 & 51428.5 & $\mid-22.9$ & 3.9 \\
\hline 43 & 18 & 114308 & 262 & 160 & 272.48 & 51390.4 & 51401.5 & $\mid-11.1$ & 51393.6 & 51416.5 & $\mid-22.9$ & 1.6 \\
\hline 43 & 18 & 114315 & 264 & 160 & 267.48 & 51379.2 & 51390.4 & $\mid-11.2$ & 51385.2 & 51408.1 & -22.9 & 3.0 \\
\hline 43 & 18 & 114323 & 266 & 160 & 262.48 & 51366.4 & 51377.7 & $\mid-11.3$ & 51370.8 & 51393.7 & -22.9 & 2.2 \\
\hline 43 & 18 & 114330 & 268 & 160 & 257.48 & 51351.2 & 51362.6 & $\mid-11.4$ & 51360.6 & $|51383.5|$ & $\mid-22.9$ & 4.7 \\
\hline
\end{tabular}




\begin{tabular}{|c|c|c|c|c|c|c|c|c|c|c|c|c|}
\hline Line & ay & Imise & La. & $\mathbf{X}$ & $\mathbf{Y}$ & ncorr. & & diff. & $\begin{array}{l}\text { BOT } \\
\text { uncorr. }\end{array}$ & $\begin{array}{l}\text { rom (nT } \\
\text { corr. }\end{array}$ & diff. & $\begin{array}{l}\text { Vert.Grad. } \\
\text { (nT/ft) }\end{array}$ \\
\hline 43 & 18 & 114339 & 270 & 160 & 252.48 & 1330.8 & 51342.2 & -11.4 & 51346.6 & 51369.5 & -22.9 & 7.9 \\
\hline 43 & 18 & 114349 & 272 & 160 & 247.48 & 51289.8 & 51301.3 & $\mid-11.5$ & 51303.2 & 51326.1 & $|-22.9|$ & 6.7 \\
\hline 43 & 18 & 114358 & 274 & 160 & 242.48 & 246.8 & 51258.4 & -11.6 & 51262.8 & 51285.6 & -22.8 & 8.0 \\
\hline 43 & 18 & 114407 & 276 & 160 & 237.48 & 80.8 & 51192.5 & -11.7 & 51188.2 & 51211.0 & -22.8 & 3.7 \\
\hline 43 & 18 & 114415 & 278 & 160 & 232.48 & 51103.8 & 51115.6 & -11.8 & 51097.8 & 51120.6 & -22.8 & -3.0 \\
\hline 43 & 18 & 114422 & 280 & 160 & 227.48 & 4.6 & 1026.5 & -11.9 & 50984.2 & 1007.0 & -22.8 & -15.2 \\
\hline 43 & 18 & 114430 & 282 & 160 & 222.48 & 50909.8 & 50921.7 & -11.9 & 50834.2 & 50857.0 & -22.8 & -37.8 \\
\hline 43 & 18 & 14437 & 284 & 160 & 217.48 & 50851.2 & 50863.2 & -12.0 & 50682.2 & 50705.0 & -22.8 & -84.5 \\
\hline 43 & 18 & 4445 & 286 & 160 & 212.48 & 50997.8 & 51009.9 & $\mid-12.1$ & 50645.2 & 50668.0 & -22.8 & -176.3 \\
\hline 43 & 18 & $\$ 52$ & 288 & 160 & 207.48 & 32.6 & 1694.8 & 12.2 & 1433.6 & 56.4 & -22.8 & -124.5 \\
\hline 43 & 18 & 114459 & 290 & 160 & 202.48 & 53123.6 & 53135.8 & -12.2 & 53624.8 & 53647.6 & -22.8 & 250.6 \\
\hline 43 & 18 & 114506 & 292 & 160 & 197.48 & 0.4 & 4452.7 & -12.3 & 58579.6 & 8602.3 & -22.7 & 2069.6 \\
\hline 43 & 18 & 114513 & 294 & 160 & 192.48 & 69.8 & 4482.2 & $\mid-12.4$ & 58574.0 & 3596.7 & $|-22.7|$ & 2052.1 \\
\hline 43 & 18 & 114520 & 296 & 160 & 187.48 & 53462.2 & 3474.6 & $\mid-12.4$ & 53947.2 & 53969.9 & -22.7 & 242.5 \\
\hline 43 & 18 & 114527 & 298 & 160 & 182.48 & 52603.8 & 52616.3 & -12.5 & 52755.0 & 52777.7 & -22.7 & 75.6 \\
\hline 43 & 18 & 114534 & 300 & 160 & 177.48 & 79.0 & 91.6 & $\mid-12.6$ & 52001.6 & 2024.3 & -22.7 & 11.3 \\
\hline 43 & 18 & 41 & 302 & .160 & 172.48 & 1.2 & 03.9 & 12.7 & 51491.4 & 514.1 & -22.7 & 0.1 \\
\hline 43 & 18 & & 304 & 160 & 167.48 & 8.8 & 31.5 & $\mid-12.7$ & 50910.6 & 933.3 & -22.7 & -4.1 \\
\hline 43 & 18 & 55 & 306 & 160 & 162.48 & 17.8 & 0260.6 & -12.8 & 49812.0 & 834.7 & -22.7 & -217.9 \\
\hline 43 & 18 & 02 & 308 & 160 & 157.48 & 92.4 & 0005.3 & -12.9 & 49359.4 & 49382.1 & -22.7 & -316.5 \\
\hline 43 & 18 & 11 & 310 & 160 & 152.48 & 2.2 & 0025.1 & $\mid-12.9$ & 58582.0 & 58604.6 & -22.6 & 284.9 \\
\hline 43 & 18 & & 312 & 160 & 147.48 & 6.8 & 459.8 & $\mid-13.0$ & 58654.8 & 3677.4 & -22.6 & 4104.0 \\
\hline 43 & 18 & & 314 & 160 & 142.48 & 51746.2 & 1759.3 & -13.1 & 51724.2 & 1746.8 & -22.6 & -11.0 \\
\hline 43 & 18 & 114632 & 316 & 160 & 137.48 & 52304.2 & 2317.4 & -13.2 & 58603.4 & 8626.0 & -22.6 & 3149.6 \\
\hline 43 & 18 & 114640 & 318 & 160 & 132.48 & 4.6 & 7.8 & -13.2 & 51751.8 & 774.4 & -22.6 & -36.4 \\
\hline 43 & 18 & 648 & 320 & 160 & 127.48 & 17.6 & 0.9 & $\mid-13.3$ & 51310.2 & 332.8 & -22.6 & -68.7 \\
\hline 43 & 18 & & 322 & 160 & 122.48 & 7.0 & 70.4 & -13.4 & 51260.8 & 283.4 & -22.6 & -48.1 . \\
\hline 44 & 18 & & 334 & 150 & 121.85 & 9.8 & 9.1 & -9.3 & 51256.4 & 278.1 & $|-21.7|$ & -31.7 \\
\hline 44 & 18 & & 336 & 150 & 126.85 & 72.8 & & -9.5 & 9.0 & 240.7 & -21.7 & -26.9 \\
\hline 44 & 18 & 115 & 338 & 150 & 131.85 & 51312.2 & 321.6 & -9.4 & 51101.8 & 123.4 & -21.6 & -105.2 \\
\hline 44 & 18 & 115341 & 340 & 150 & 136.85 & 51638.0 & 1647.3 & -9.3 & 52308.8 & 52330.4 & -21.6 & 335.4 \\
\hline 44 & 18 & 48 & 342 & 150 & 141.85 & 99.2 & 308.4 & -9.2 & 51310.0 & 51331.6 & -21.6 & 5.4 \\
\hline 44 & 18 & 15356 & 344 & 150 & 146.85 & 50957.8 & 966.9 & -9.1 & 51290.6 & 1312.2 & -21.6 & 166.4 \\
\hline 44 & 18 & 04 & 346 & 150 & 151.85 & 77.6 & 36.6 & -9.0 & 54004.8 & 4026.4 & -21.6 & 1788.6 \\
\hline 44 & 18 & & 348 & 150 & 156.85 & & & -8.9 & 50063.0 & 084.5 & -21.5 & -135.6 \\
\hline 44 & 18 & & 350 & 150 & 85 & & & -8.8 & 7.8 & 19.3 & -21.5 & -139.9 \\
\hline 44 & 18 & & 352 & 150 & & 3.0 & & -8.7 & 51154.4 & 175.9 & -21.5 & -4.3 \\
\hline 44 & 18 & 33 & 354 & 150 & 171.85 & 51621.6 & 630.3 & -8.7 & 51616.4 & 1637.9 & -21.5 & -2.6 \\
\hline 44 & .18 & 115440 & 356 & 150 & 176.85 & 52125.0 & 2133.6 & -8.6 & 52097.4 & 52118.9 & -21.5 & -13.8 \\
\hline 44 & 18 & 47 & 358 & 150 & 181.85 & 52868.6 & 8877.1 & -8.5 & 52923.6 & 945.1 & $\mid-21.5$ & 27.5 \\
\hline 44 & 18 & 54 & 360 & 150 & 186.85 & 53920.6 & 929.0 & -8.4 & 54386.6 & 408.0 & $|-21.4|$ & 233.0 \\
\hline 44 & 18 & 01 & 362 & 150 & 191.85 & 55029.0 & 7.3 & -8.3 & 58539.4 & 560.8 & $|-21.4|$ & 755.2 \\
\hline 44 & 18 & & 364 & 150 & 196.85 & 54587.6 & 5.8 & -8.2 & 58630.2 & 651.6 & $|-21.4|$ & 2021.3 \\
\hline 44 & 18 & 115516 & 366 & 150 & 201,85 & 52812.8 & 2820.9 & -8.1 & 52887.6 & 52909.0 & -21.4 & 37.4 \\
\hline 44 & 18 & 115523 & 368 & 150 & 206.85 & 51281.4 & 1289.4 & -8.0 & 50892.8 & 0914.2 & $|-21.4|$ & -194.3 \\
\hline 44 & 18 & 115531 & 370 & 150 & 211.85 & 50590.0 & 50597.9 & -7.9 & 49992.0 & 50013.3 & $|-21.3|$ & -299.0 \\
\hline 44 & 18 & 115538 & 372 & 150 & 216.85 & 50519.2 & 50527.1 & -7.9 & 50212.0 & 50233.3 & -21.3 & -153.6 \\
\hline 44 & 18 & 115545 & 374 & 150 & 221.85 & 50701.0 & 50708.8 & -7.8 & 50566.4 & 50587.7 & -21.3 & -67.3 \\
\hline 44 & 18 & 115552 & 376 & 150 & 226.85 & 50897.0 & 50904.7 & -7.7 & $50838.6^{\circ}$ & |50859.9 & $\mid-21.3$ & -29.2 \\
\hline
\end{tabular}




\begin{tabular}{|c|c|c|c|c|c|c|c|c|c|c|c|c|}
\hline Line & Day & Time & Sta. & $\mathbf{X}$ & $\mathbf{Y}$ & uncorr. & $\begin{array}{l}\text { (nT) } \\
\text { corr. }\end{array}$ & diff. & $\begin{array}{l}\mathrm{BO} 7 \\
\text { uncorr. }\end{array}$ & $\begin{array}{l}\text { TOM (nT } \\
\text { corr. }\end{array}$ & diff. & $\begin{array}{c}\text { Vert.Grad. } \\
\text { (nT/ft) }\end{array}$ \\
\hline 44 & 18 & 115558 & 378 & 150 & 231.85 & 51044.2 & 51051.8 & -7.6 & 51012.6 & 51033.9 & -21.3 &,-15.8 \\
\hline 44 & 18 & 115605 & 380 & 150 & 236.85 & 51133.0 & 51140.5 & -7.5 & 51122.0 & 51143.3 & $\mid-21.3$ & -5.5 \\
\hline 44 & 18 & 115611 & 382 & 150 & 241.85 & 51203.4 & 51210.8 & -7.4 & 51214.0 & 51235.2 & $|-21.2|$ & 5.3 \\
\hline 44 & 18 & 115619 & 384 & 150 & 246.85 & 51269.4 & 51276.7 & -7.3 & 51283.8 & 51305.0 & $|-21.2|$ & 7.2 \\
\hline 44 & 18 & 115630 & 386 & 150 & 251.85 & 51312.6 & 51319.8 & -7.2 & 51314.8 & 51336.0 & $|-21.2|$ & 1.1 \\
\hline 44 & 18 & 115638 & 388 & 150 & 256.85 & 51342.2 & 51349.3 & -7.1 & 51347.6 & 51368.8 & $|-21.2|$ & 2.7 \\
\hline 44 & 18 & 115646 & 390 & 150 & 261.85 & .51365 .8 & 51372.8 & -7.0 & 51369.8 & 51391.0 & $|-21.2|$ & 2.0 \\
\hline 44 & 18 & 115654 & 392 & 150 & 266.85 & 51380.8 & 51387.7 & -6.9 & 51387.0 & 51408.1 & $|-21.1|$ & 3.1 \\
\hline 44 & 18 & 115704 & 394 & 150 & 271.85 & 51394.4 & 51401.2 & -6.8 & 51399.8 & 51420.9 & $|-21.1|$ & 2.7 \\
\hline 44 & 18 & 115712 & 396 & 150 & 276.85 & 51408.2 & 51414.9 & -6.7 & 51411.4 & 51432.5 & $\mid-21.1$ & 1.6 \\
\hline 44 & 18 & 115720 & 398 & 150 & 281.85 & 51416.2 & 51422.8 & -6.6 & 51421.2 & 51442.3 & -21.1 & 2.5 \\
\hline 44 & 18 & 115728 & $400^{\circ}$ & 150 & 286.85 & 51418.8 & 51425.3 & -6.5 & 51419.6 & 51440.7 & $|-21.1|$ & 0.4 \\
\hline 44 & 18 & 115735 & 402 & 150 & 291.85 & 51424.8 & $\mid 51431.2$ & -6.4 & 51427.6 & 51448.6 & $|-21.0|$ & 1.4 \\
\hline 44 & 18 & 115743 & 404 & 150 & 296.85 & 51431.2 & 51437.5 & -6.3 & 51430.2 & 51451.2 & $|-21.0|$ & -0.5 \\
\hline 44 & 18 & 15751 & 406 & 150 & 301.85 & 51436.0 & 51442.2 & -6.2 & 51434.4 & 51455.4 & $|-21.0|$ & -0.8 \\
\hline 44 & 18 & 15801 & 408 & 150 & 306.85 & 51441.8 & 51447.9 & -6.1 & 51439.4 & $51460.4 \mid$ & $|-21.0|$ & -1.2 \\
\hline 44 & 18 & 15809 & 410 & 150 & 311.85 & 51443.8 & 51449.8 & -6.0 & 51442.2 & 51463.2 & $|-21.0|$ & -0.8 \\
\hline 45 & 18 & 20159 & 412 & 140 & 311.22 & 51437.8 & 51440.9 & -3.1 & 51443.2 & 51463.6 & $|-20.4|$ & 2.7 \\
\hline 45 & 18 & 120207 & 414 & 140 & 306.22 & 51436.0 & 51439.0 & -3.0 & 51442.0 & 51462.4 & -20.4 & 3.0 \\
\hline 45 & 18 & 120214 & 416 & 140 & 301.22 & 51433.4 & 51436.4 & -3.0 & 51439.0 & 51459.4 & -20.4 & 2.8 \\
\hline 45 & 18 & 120221 & 418 & 140 & 296.22 & 51428.6 & 51431.5 & -2.9 & 51437.6 & 51457.9 & -20.3 & 4.5 \\
\hline 45 & 18 & 120228 & 420 & 140 & 291.22 & .51426 .4 & 51429.2 & -2.8 & 51428.4 & 51448.7 & $\mid-20.3$ & 1.0 \\
\hline 45 & 18 & 120235 & 422 & 140 & 286.22 & 5.1425 .8 & 51428.5 & -2.7 & 51423.0 & 51443.3 & $\mid-20.3$ & -1.4 \\
\hline 45 & 18 & 120242 & 424 & 140 & 281.22 & 51414.4 & $51417.0 \mid$ & -2.6 & 51421.6 & 51441.9 & -20.3 & 3.6 \\
\hline 45 & 18 & 120249 & 426 & 140 & 276.22 & 51405.4 & 51407.9 & -2.5 & 51413.8 & 51434.1 & -20.3 & 4.2 \\
\hline 45 & 18 & 120256 & 428 & 140 & 271.22 & 51394.6 & 51397.0 & -2.4 & 51404.6 & 51424.9 & -20.3 & 5.0 \\
\hline 45 & 18 & 120303 & 430 & 140 & 266.22 & 51381.6 & $|51384.0|$ & -2.4 & 51394.0 & 51414.2 & -20.2 & 6.2 \\
\hline 45 & 18 & 120310 & 432 & 140 & 261.22 & 51365.4 & 51367.7 & -2.3 & 51380.2 & 51400.4 & -20.2 & 7.4 \\
\hline 45 & 18 & 120317 & 434 & 140 & 256.22 & 51341.6 & $|51343.8|$ & -2.2 & 51343.2 & 51363.4 & -20.2 & 0.8 \\
\hline 45 & .18 & 120326 & 436 & 140 & 251.22 & 51305.4 & 51307.5 & -2.1 & 51321.2 & 51341.4 & -20.2 & 7.9 \\
\hline 45 & 18 & 120333 & 438 & 140 & 246.22 & 51234.0 & 51236.0 & -2.0 & 51248.0 & 51268.2 & -20.2 & 7.0 \\
\hline 45 & 18 & 120340 & 440 & 140 & 241.22 & 51 & 13.7 & -1.9 & $5 \nmid 067.4$ & 51087.5 & $\mid-20.1$ & -22.2 \\
\hline 45 & 18 & 120347 & 442 & 140 & 236.22 & 51060.8 & $|51062.6|$ & -1.8 & 51054.0 & 51074.1 & $-20.1 \mid$ & -3.4 \\
\hline 45 & 18 & 120354 & 444 & 140 & 231.22 & 50933.8 & 50935.5 & --1.7 & 50950.0 & 50970.1 & $-20.1 \mid$ & 8.1 \\
\hline 45 & 18 & 120401 & 446 & 140 & 226.22 & 50766.8 & $|50768.4|$ & -1.6 & 50734.4 & 50754.5 & $-20.1 \mid$ & -16.2 \\
\hline 45 & 18 & 120407 & 448 & 140 & 221.22 & 50484.4 & 50486.0 & -1.6 & 50375.2 & 50395.3 & -20.1 & -54.6 \\
\hline 45 & 18 & 120414 & 450 & 140 & 216.22 & 50154.8 & 50156.3 & -1.5 & 49828.4 & 49848.5 & -20.1 & -163.2 \\
\hline 45 & 18 & 120422 & 452 & 140 & 211.22 & 49998.4 & |49999:8 & -1.4 & 49306.2 & 49326.2 & -20.0 & -346.1 \\
\hline 45 & 18 & 120429 & 454 & 140 & 206.22 & 50585.4 & $|50586.7|$ & -1.3 & 49882.4 & 49902.4 & -20.0 & -351.5 \\
\hline 45 & 18 & 120436 & 456 & 140 & 201.22 & 51759.2 & 51760.4 & -1.2 & 51098.2 & 51118.2 & -20.0 & -330.5 \\
\hline 45 & 18 & 120444 & 458 & 140 & 196.22 & 53724.0 & 53725.1 & -1.1 & 54356.8 & 54376.8 & -20.0 & 316.4 \\
\hline 45 & 18 & 120451 & 460 & 140 & 191.22 & 54588.8 & 54589.8 & -1.0 & 56158.2 & 56178.2 & -20.0 & 784.7 \\
\hline 45 & 18 & 120458 & 462 & 140 & 186.22 & 53593.6 & 53594.5 & -0.9 & 54136.0 & 54156.0 & -20.0 & 271.2 \\
\hline 45 & 18 & 120506 & 464 & 140 & 181.22 & 52577.0 & 52577.8 & -0.8 & 52661.4 & 52681.3 & -19.9 & 42.2 \\
\hline 45 & 18 & 120512 & 466 & 140 & 176.22 & 51990.4 & 51991.2 & -0.8 & 51947.2 & 51967.1 & $\mid-19.9$ & -21.6 \\
\hline 45 & 18 & 120519 & 468 & 140 & 171.22 & 51646.0 & $|51646.7|$ & -0.7 & 51631.2 & 51651.1 & $\mid-19.9$ & -7.4 \\
\hline 45 & 18 & 120526 & 470 & 140 & 166.22 & 51378.6 & $\mid 51379.2$ & -0.6 & 51406.0 & 51425.9 & $\mid-19.9$ & 13.7 \\
\hline 45 & 18 & 120533 & 472 & 140 & 161.22 & 50999.0 & $|50999.5|$ & -0.5 & 51000.6 & 51020.5 & $|-19.9|$ & 0.8 \\
\hline 15 & 18 & 120541 & 474 & 140 & 156.22 & 50215.8 & 50216.2 & -0 & 58568.4 & 58588.3 & -19.5 & 4176.3 \\
\hline
\end{tabular}




\begin{tabular}{|c|c|c|c|c|c|c|c|c|c|c|c|c|}
\hline & & & & & & & T) & & BO & ON & & \\
\hline Line & ay & Time & ta. & $\mathbf{x}$ & $\mathbf{Y}$ & uncorr. & corr. & diff. & ncorr. & corr. & diff. & \\
\hline 45 & 18 & 120548 & 476 & 140 & 151.22 & 50044.0 & 50044.3 & -0.3 & 49470.2 & 49490.0 & -19.8 & -286.9 \\
\hline 45 & 18 & 120556 & 478 & 140 & 146.22 & 50487.4 & 50487.6 & -0.2 & 50529.2 & 50549.0 & -19.8 & 20.9 \\
\hline 45 & 18 & 120604 & 480 & 140 & 141.22 & 50824.4 & 50824.5 & -0.1 & 50593.2 & 50618.0 & $\mid-19.8$ & -115.6 \\
\hline 45 & 18 & 120611 & 482 & 140 & 136.22 & 51152.4 & 51152.4 & 0.0 & 51332.4 & 51352.2 & $\mid-19.8$ & 90.0 \\
\hline 45 & 18 & 120619 & 484 & 140 & 131.22 & 51194.2 & 51194.1 & 0.1 & 50902.4 & 50922.2 & $\mid-19.8$ & -145.9 \\
\hline 45 & 18 & 120626 & 486 & 140 & 126.22 & 51398.4 & 51398.2 & 0.2 & 51008.0 & 51027.7 & $\mid-19.7$ & -195.2 \\
\hline 45 & 18 & 120635 & 488 & 140 & 121.22 & 51418.0 & 51417.7 & 0.3 & 51359.0 & 51378.7 & $\mid-19.7$ & -29.5 \\
\hline 46 & 18 & 121334 & 500 & 130 & 120.59 & 52262.6 & 52262.8 & -0.2 & 53071.8 & 53091.0 & -19.2 & 404.6 \\
\hline 46 & 18 & 121344 & 502 & 130 & 125.59 & 51562.4 & 51562.1 & 0.3 & 51459.2 & 51478.3 & $\mid-19.1$ & -51.6 \\
\hline 46 & 18 & 121352 & 504 & 130 & 130.59 & 51188.8 & 51188.5 & 0.3 & 51081.4 & 51100.5 & -19.1 & -53.7 \\
\hline 46 & 18 & 121359 & 506 & 130 & 135.59 & 51113.0 & 1112.8 & 0.2 & 51026.6 & 51045.7 & $\mid-19.1$ & -43.2 \\
\hline 46 & 18 & 121405 & 508 & 130 & 140.59 & 51061.0 & 51060.8 & 0.2 & 50994.8 & 51013.9 & $\mid-19.1$ & -33.1 \\
\hline 46 & 18 & 121412 & 510 & 130 & 145.59 & 51029.8 & 51029.6 & 0.2 & 50992.6 & 51011.7 & $\mid-19.1$ & -18.6 \\
\hline 46 & 18 & 121418 & 512 & 130 & 150.59 & 51068.6 & 51068.4 & 0.2 & 51112.0 & 51131.1 & $\mid-19.1$ & 21.7 \\
\hline 46 & 18 & 121425 & 514 & 130 & 155.59 & 51179.4 & 51179.2 & 0.2 & 51261.0 & 51280.1 & -19.1 & 40.8 \\
\hline 46 & 18 & 121432 & 516 & 130 & 160.59 & 51329.8 & 51329.6 & 0.2 & 51357.0 & 51376.1 & $\mid-19.1$ & 13.6 \\
\hline 46 & 18 & 121438 & 518 & 130 & 165.59 & 49.4 & 449.2 & 0.2 & 51398.6 & 17.7 & $\mid-19.1$ & -25.4 \\
\hline 46 & 18 & 121445 & 520 & 130 & 170.59 & 51640.4 & 1640.2 & 0.2 & 51445.2 & 51464.3 & $|-19.1|$ & -97.6 \\
\hline 46 & 18 & 121451 & 522 & 130 & 175.59 & 51870.2 & 51870:0 & 0.2 & 51642.2 & 51661.3 & $\mid-19.1$ & -114.0 \\
\hline 46 & 18 & 121458 & 524 & 130 & 180.59 & 52233.0 & 52232.8 & 0.2 & 52136.4 & 52155.5 & $\mid-19.1$ & -48.3 \\
\hline 46 & 18 & 121505 & 526 & 130 & 185.59 & 53158.4 & 53158.3 & 0.1 & 53361.8 & 53380.8 & -19.0 & 101.7 \\
\hline 46 & 18 & 121512 & 528 & 130 & 190.59 & 54500.8 & 54500.7 & 0.1 & 35909.2 & 35928.2 & -19.0 & -9295.8 \\
\hline 46 & 18 & 121518 & 530 & 130 & 195.59 & 54544.8 & 54544.7 & 0.1 & 17754.8 & 17773.8 & -19.0 & -18395.0 \\
\hline 46 & 18 & 121526 & 532 & 130 & 200.59 & 52294.0 & 52293.9 & 0.1 & 52659.4 & 52678.4 & $\mid-19.0$ & 182.7 \\
\hline 46 & 18 & 121533 & 534 & 130 & 205.59 & 50473.6 & 50473.5 & 0.1 & 49701.2 & 49720.2 & $\mid-19.0$ & -386.2 \\
\hline 46 & 18 & 121540 & 536 & 130 & 210.59 & 49813.6 & 49813.5 & 0.1 & 48975.8 & 48994.8 & -19.0 & -418.9 \\
\hline 46 & 18 & 121547 & 538 & 130 & 215.59 & 50002.6 & 50002.5 & 0.1 & 49606.8 & 49625.8 & $\mid-19.0$ & -197.9 \\
\hline 46 & 18 & 121554 & 540 & 130 & 220.59 & 50484.8 & 50484.7 & 0.1 & 50278.6 & 50297.6 & -19.0 & -103.1 \\
\hline 46 & 18 & 121600 & 542 & 130 & 225.59 & 50760.6 & 50760.6 & 0.0 & 50708.2 & 50727.2 & $\mid-19.0$ & -26.2 \\
\hline 46 & 18 & 121606 & 544 & 130 & 230.59 & 50974.8 & 50974.8 & 0.0 & 50970.0 & 50989.0 & $\mid-19.0$ & -2.4 \\
\hline 46 & 18 & 121612 & 546 & 130 & 235.59 & 51167.4 & 51167.4 & 0.0 & 51211.4 & 51230.4 & $\mid-19.0$ & 22.0 \\
\hline 46 & 18 & 121619 & 548 & 130 & 240.59 & 51320.0 & 51320.0 & 0.0 & 51528.0 & 51547.0 & -19.0 & 104.0 \\
\hline 46 & 18 & 121626 & 550 & 130 & 245.59 & 51300.0 & 51300.0 & 0.0 & 51304.2 & 51323.1 & $\mid-18.9$ & 2.1 \\
\hline 46 & 18 & 121633 & 552 & 130 & 250.59 & 51318.0 & 513.18 .0 & 0.0 & 51328.4 & 51347.3 & -18.9 & 5.2 \\
\hline 46 & 18 & 121640 & 554 & 130 & 255.59 & 54.4 & 54.4 & 0.0 & 51357.2 & 51376.1 & $\mid-18.9$ & 1.4 \\
\hline 46 & 18 & 121647 & 556 & 130 & 260.59 & 51384.6 & 384.6 & 0.0 & 51383.8 & 51402.7 & -18.9 & -0.4 \\
\hline 46 & 18 & 121653 & 558 & 130 & 265.59 & 51398:6 & 51398.6 & 0.0 & 51397.2 & 416.1 & -18.9 & -0.7 \\
\hline 46 & 18 & 121659 & 560 & 130 & 270.59 & 51409.8 & 51409.8 & 0.0 & 51407.8 & 426.7 & $\mid-18.9$ & -1.0 \\
\hline 46 & 18 & 121705 & 562 & 130 & 275.59 & 51420.6 & 51420.7 & -0.1 & 51417.2 & 51436.1 & $\mid-18.9$ & -1.7 \\
\hline 46 & 18 & 121711 & 564 & 130 & 280.59 & 51429.8 & 51429.9 & -0.1 & 51425.0 & 51443.9 & $\mid-18.9$ & -2.4 \\
\hline 46 & 18 & 121717 & 566 & 130 & 285.59 & 51436.4 & 51436.5 & -0.1 & 51432.8 & 51451.7 & $\mid-18.9$ & -1.8 \\
\hline 46 & 18 & 121723 & 568 & 130 & 290.59 & 51436.4 & 51436.5 & -0.1 & 51432.0 & 51450.9 & $\mid-18.9$ & -2.2 \\
\hline 46 & 18 & 121730 & 570 & 130 & 295.59 & 51441.0 & 51441.1 & -0.1 & 51437.4 & 51456.3 & -18.9 & -1.8 \\
\hline 46 & 18 & 121735 & 572 & 130 & 300.59 & 51443.4 & 51443.5 & -0.1 & 51438.2 & 51457.1 & -18.9 & -2.6 \\
\hline 46 & 18 & 121741 & 574 & 130 & 305.59 & 51449.0 & 51449.1 & -0.1 & 51439.6 & 51458.5 & $\mid-18.9$ & -4.7 \\
\hline 46 & 18 & 121747 & 576 & 130 & 310.59 & 51453.4 & 51453.5 & -0.1 & 51445.4 & 51464.3 & $\mid-18.9$ & -4.0 \\
\hline 47 & 18 & 122416 & 578 & 120 & 309.96 & 51447.0 & 51447.8 & -0.8 & 51457.6 & 476.0 & $\mid-18.4$ & 5.3 \\
\hline 47 & 18 & 122422 & 580 & 120 & 304.96 & 51444.6 & 51445.4 & -0.8 & 51450.8 & 51469.2 & $\mid-18.4$ & 3.1 \\
\hline 47 & 18 & 122429 & 582 & 120 & 299.96 & 51439.2 & 51440.0 & -0.8 & 448.6 & | 51467.0 & 8.4 & 4.7 \\
\hline
\end{tabular}




\begin{tabular}{|c|c|c|c|c|c|c|c|c|c|c|c|c|}
\hline & & & & & & & (nT) & & $B C$ & TOM $\left(n^{\circ}\right)$ & & \\
\hline Line & Day & Time & Sta. & $x$ & $\mathbf{Y}$ & uncorr. & corr. & diff. & uncorr. & corr. & diff. & \\
\hline 47 & 18 & 122435 & 584 & 120 & 294.96 & 51442.0 & 51442.8 & -0.8 & 51444.4 & 51462.8 & -18.4 & 1.2 \\
\hline 47 & 18 & 2442 & 586 & 120 & 39.96 & 51440.0 & 51440.8 & -0.8 & 452.2 & 51470.5 & -18.3 & 6.1 \\
\hline 47 & 18 & 122451 & 588 & $120 \mid$ & 284.96 & 51434.4 & 51435.2 & -0.8 & 51449.2 & 51467.5 & $|-18.3|$ & 7.4 \\
\hline 47 & 18 & 122458 & 590 & $|120|$ & 279.96 & 51427.6 & 51428.4 & -0.8 & 51436.4 & 51454.7 & $|-18.3|$ & 4.4 \\
\hline 47 & 18 & 122505 & 592 & 120 & 274.96 & 51416.4 & 51417.3 & -0.9 & 51423.8 & 51442.1 & $\mid-18.3$ & 3.7 \\
\hline 47 & 18 & 122512 & 594 & 120 & 269.96 & 51407.0 & 51407.9 & -0.9 & 51418.0 & 1436.3 & $\mid-18.3$ & 5.5 \\
\hline 47 & 18 & 122518 & 596 & 120 & 264.96 & 51397.0 & 51397.9 & -0.9 & 51410.0 & 51428.3 & $\mid-18.3$ & 6.5 \\
\hline 47 & 18 & -122526 & 598 & $120 \mid$ & | 259.96 & 51382.4 & 51383.3 & -0.9 & 51400.6 & 51418.9 & $\mid-18.3$ & 9.1 \\
\hline 47 & 18 & 122533 & 600 & $\mid 120$ & 254.96 & 51362.2 & 51363.1 & -0.9 & 51379.8 & |51398.1 & $\mid-18.3$ & 8.8 \\
\hline 47 & 18 & 122540 & 602 & $|120|$ & 249.96 & 51329.8 & 51330.7 & -0.9 & 51343.6 & 51361.9 & $\mid-18.3$ & 6.9 \\
\hline 47 & 18 & 122547 & 604 & 120 & 244.96 & 51286.8 & 51287.7 & -0.9 & 51304.6 & 51322.9 & $|-18.3|$ & 8.9 \\
\hline 47 & 18 & 122555 & 606 & 120 & 239.96 & 5.1228 .6 & 51229.5 & -0.9 & 51246.6 & 51264.9 & -18.3 & 9.0 \\
\hline 47 & 18 & 122601 & 608 & .120 & 234.96 & 51136.0 & 136.9 & -0.9 & 51156.8 & 51175.1 & $\mid-18.3$ & 10.4 \\
\hline 47 & 18 & 122608 & 610 & $120 \mid$ & 229.96 & 33.2 & 994.2 & -1.0 & 07.8 & 26.0 & $\mid-18.2$ & 7.3 \\
\hline 47 & 18 & 122614 & 612 & $|120|$ & 224.96 & 50799.4 & 50800.4 & -1.0 & 50792.4 & 50810.6 & $\mid-18.2$ & -3.5 \\
\hline 47 & 18 & 122621 & 614 & 120 & 219.96 & 50528.0 & 50529.0 & -1.0 & 50442.6 & 0460.8 & -18.2 & -42.7 \\
\hline 47 & 18 & 22629 & 616 & 120 & 214.96 & 04.0 & 205.0 & -1.0 & 41.0 & 959.2 & $\mid-18.2$ & -131.5 \\
\hline 47 & 18 & 122636 & 618 & $120 \mid$ & 209.96 & 55.8 & 49966.8 & -1.0 & 49379.8 & 398.0 & $\mid-18.2$ & -293.0 \\
\hline 47 & 18 & 122643 & 620 & $120 \mid$ & 204.96 & 50096.2 & 097.2 & -1.0 & 38.6 & 9356.8 & $\mid-18.2$ & -378.8 \\
\hline 47 & 18 & 122651 & 622 & 120 & 199.96 & 50792.2 & 50793.2 & -1.0 & 50107.4 & 0125.6 & $\mid-18.2$ & -342.4 \\
\hline 47 & 18 & 122657 & 624 & 120 & 194.96 & 51865.0 & 51866.0 & -1.0 & 01.4 & 1719.6 & -18.2 & -81.8 \\
\hline 47 & 18 & 122705 & 626 & $|120|$ & 189.96 & 52318.2 & 52319.3 & -1.1 & 52402.2 & 2420.4 & $\mid-18.2$ & 42.0 \\
\hline 47 & 18 & 122712 & 628 & $120 \mid$ & 184.96 & 51985.6 & 51986.7 & -1.1 & 51911.2 & 51929.4 & $\mid-18.2$ & -37.2 \\
\hline 47 & 18 & 122719 & 630 & 120 & 179.96 & 31.4 & 51682.5 & -1.1 & 64.4 & 1582.6 & $\mid-18.2$ & -58.5 \\
\hline 47 & 18 & 122726 & 632 & 120 & 174.96 & 0.8 & 451.9 & -1.1 & 62.4 & 380.6 & -18.2 & -44.2 \\
\hline 47 & 18 & 122732 & 634 & $120 \mid$ & 169.96 & 2.8 & 373.9 & -1.1 & 33.8 & 201.9 & $\mid-18.1$ & -94.5 \\
\hline 47 & 18 & 122739 & 636 & 120 & 164.96 & 6.4 & 51377.5 & -1.1 & 5.4 & 323.5 & $\mid-18.1$ & -35.5 \\
\hline 47 & 18 & 122746 & 638 & $|120|$ & 159.96 & 51364.6 & 51365.7 & -1.1 . & 51359.6 & 51377.7 & $\mid-18.1$ & -2.5 \\
\hline 47 & 18. & 122753 & 640 & 120 & 154.96 & 51343.4 & 344.5 & -1.1 & 51355.0 & 1373.1 & -18.1 & 5.8 \\
\hline 47 & 18 & 122800 & 642 & 120 & 149.96 & 51313.0 & 51314.1 & -1.1 & 51317.8 & 1335.9 & $\mid-18.1$ & 2.4 \\
\hline 47 & 18 & 122808 & 644 & 120 & 144.96 & 51289.2 & 51290.4 & -1.2 & 51307.6 & 51325.7 & $\mid-18.1$ & 9.2 \\
\hline 47 & 18 & 122814 & 646 & $120 \mid$ & 139.96 & 51265.0 & 51266.2 & -1.2 & 51284.6 & 51302.7 & $|-18.1|$ & 9.8 \\
\hline 47 & 18 & 122823 & 648 & 120 & 134.96 & 51212.6 & 51213.8 & -1.2 & 51218.0 & 1236.1 & $|-18.1|$ & 2.7 \\
\hline 47 & 18 & 122829 & 650 & 120 & 129.96 & 17.6 & 48.8 & -1.2 & 0.8 & 148.9 & -18.1 & -8.4 \\
\hline 47 & 18 & 122837 & 652 & $120 \mid$ & 124.96 & 1.0 & 50992.2 & -1.2 & 5.2 & 50863.3 & $\mid-18.1$ & -72.9 \\
\hline 47 & 18 & 122845 & 654 & 120 & 119.96 & 51124.0 & 51125.2 & -1.2 & 38.6 & 58616.7 & $\mid-18.1$ & 3737.3 \\
\hline 48 & 18 & 123444 & 666 & $|110|$ & 119.33 & 49983.8 & 49984.9 & -1.1 & 53079.6 & 3098.6 & $|-19.0|$ & 1547.9 \\
\hline 48 & 18 & 123452 & 668 & 110 & 124.33 & 50663.8 & 50665.0 & -1.2 & 58571.0 & 58590.0 & -19.0 & 3953.6 \\
\hline 48 & 18 & 123500 & 670 & $110 \mid$ & 129.33 & 51236.4 & 51237.6 & -1.2 & 51364.0 & 1383.0 & $\mid-19.0$ & 63.8 \\
\hline 48 & 18 & 123507 & 672 & 110 & 134.33 & 51312.4 & 51313.6 & -1.2 & 51350.0 & 51369.0 & $\mid-19.0$ & $18: 8$ \\
\hline 48 & 18 & 123515 & 674 & 110 & 139.33 & 51336.0 & 51337.2 & -1.2 & 51360.8 & 51379.9 & $\mid-19.1$ & 12.4 \\
\hline 48 & 18 & 123523 & 676 & 110 & 144.33 & 51347.8 & 51349.0 & -1.2 & 51365.2 & 51384.3 & $|-19.1|$ & 8.7 \\
\hline 48 & 18 & 123529 & 678 & $110 \mid$ & 149.33 & 51357.0 & 358.2 & -1.2 & 51365.0 & 384.1 & $\mid-19.1$ & 4.0 \\
\hline 48 & 18 & 123535 & 680 & 110 & 154.33 & 51353.2 & 51354.4 & -1.2 & 51362.8 & 51381.9 & $\mid-19.1$ & 4.8 \\
\hline 48 & 18 & 123542 & 682 & $110 \mid$ & 159.33 & 51348.6 & 51349.8 & -1.2 & 51343.0 & 51362.2 & $\mid-19.2$ & -2.8 \\
\hline 48 & 18 & 123549 & 684 & 110 & 164.33 & 51356.0 & 51357.2 & -1.2 & 51328.6 & 51347.8 & $\mid-19.2$ & -13.7 \\
\hline 48 & 18 & 123555 & $686^{\circ}$ & $110 \mid$ & 169.33 & 51359.6 & 51360.7 & -1.1 & 51318.4 & 51337.6 & $|-19.2|$ & -20.6 \\
\hline 48 & 18 & 123602 & 688 & 110 & 174.33 & 51393.0 & 51394.1 & -1.1 & 51312.4 & 51331.6 & $\mid-19.2$ & -40.3 \\
\hline 48 & 18 & 123607 & 690 & $|110|$ & 179.33 & 51456.8 & 51457.9 & -1.1 & 51351.0 & | 51370.2 & $\mid-19.2$ & -52.9 \\
\hline
\end{tabular}




\begin{tabular}{|c|c|c|c|c|c|c|c|c|c|c|c|c|}
\hline ne & av & me & ta. & $\mathbf{X}$ & $Y$ & ICort. & T) & III. & BOT & TOM (nT) & ditt. & $\begin{array}{l}\text { Vert.Grad. } \\
\text { (nTfft) }\end{array}$ \\
\hline 48 & 18 & 123613 & 692 & 110 & 184.33 & 51542.0 & 51543.1 & -1.1 & 51452.6 & 51471.8 & -19.2 & -44.7 \\
\hline 48 & 18 & 123619 & 694 & 110 & 189.33 & 51603.8 & 51604.9 & -1.1 & 51594.8 & 51614.1 & -19.3 & -4.5 \\
\hline 48 & 18 & 123626 & 696 & 110 & 194.33 & 51466.0 & 51467.1 & -1.1 & 51410.2 & 51429.5 & -19.3 & -27.9 \\
\hline 48 & 18 & 123633 & 698 & 110 & 199.33 & 51154.8 & 51155.9 & -1.1 & 50975.6 & 50994.9 & $\mid-19.3$ & -89.6 \\
\hline 48 & 18 & 123641 & 700 & 110 & 204.33 & 50767.6 & 50768.7 & -1.1 & 50558.6 & 50577.9 & $\mid-19.3$ & -104.5 \\
\hline 48 & 18 & 123648 & 702 & 110 & 209.33 & 50412.6 & 50413.7 & -1.1 & 49995.6 & 50015.0 & $\mid-19.4$ & -208.5 \\
\hline 48 & 18 & 123654 & 704 & 110 & 214.33 & 50648.2 & 50649.3 & -1.1 & 50419.6 & 50439.0 & $\mid-19.4$ & -114.3 \\
\hline 48 & 18 & 123702 & 706 & 110 & 219.33 & 50612.0 & 50613.1 & -1.1 & 50363.4 & 50382.8 & $\mid-19.4$ & -124.3 \\
\hline 48 & 18 & 123708 & 708 & 110 & 224.33 & 50839.6 & 50840.7 & $-1 . i$ & 50792.2 & 50811.6 & -19.4 & -23.7 \\
\hline 48 & 18 & 123715 & 710 & 110 & 229.33 & 51042.8 & 51043.9 & -1.1 & 51028.6 & 51048.0 & $\mid-19.4$ & -7.1 \\
\hline 48 & 18 & 123721 & 712 & 110 & 234.33 & 51173.8 & 51174.9 & -1.1 & 51177.0 & 51196.5 & $\mid-19.5$ & 1.6 \\
\hline 48 & 18 & 123728 & 714 & 110 & 239.33 & 51252.4 & 51253.5 & -1.1 & 51260.2 & 51279.7 & -19.5 & 3.9 \\
\hline 48 & 18 & 123734 & 716 & 110 & 244.33 & 51312.0 & 51313.1 & -1.1 & 51317.0 & 51336.5 & -19.5 & 2.5 \\
\hline 48 & 18 & 123741 & 718 & 110 & 249.33 & 51344.8 & 51345.9 & -1.1 & 51353.0 & 51372.5 & -19.5 & 4.1 \\
\hline 48 & 18 & 123748 & 720 & 110 & 254.33 & 51371.4 & 51372.5 & -1.1 & 51378.0 & 51397.5 & -19.5 & 3.3 \\
\hline 48 & 18 & 123759 & 722 & 110 & 259.33 & 51388.2 & 51389.3 & -1.1 & 51405.8 & 51425.4 & -19.6 & 8.8 \\
\hline 48 & 18 & 123809 & 724 & 110 & 264.33 & 51403.2 & 51404.3 & -1.1 & 51415.2 & 51434.8 & -19.6 & 6.0 \\
\hline 48 & 18 & 123817 & 726 & 110 & 269.33 & 51414.4 & 51415.5 & -1.1 & 51430.8 & 51.450 .4 & -19.6 & 8.2 \\
\hline 48 & 18 & 123824 & 728 & 110 & 274.33 & 51427.0 & 51428.1 & -1.1 & 51434.0 & 51453.7 & $\mid-19.7$ & 3.5 \\
\hline 48 & 18 & 123832 & 730 & 110 & 279.33 & 51432.4 & 51433.5 & -1.1 & 51433.2 & 51452.9 & -19.7 & 0.4 \\
\hline 48 & 18 & 123839 & 732 & 110 & 284.33 & 51437.8 & 51438.9 & -1.1 & 51439.8 & 59.5 & $\mid-19.7$ & 1.0 \\
\hline 48 & 18 & 123847 & 734 & 110 & 289.33 & 51446.6 & 51447.7 & -1.1 & 51445.2 & 64.9 & $\mid-19.7$ & -0.7 \\
\hline 48 & 18 & 123856 & 736 & 110 & 294.33 & 51445.6 & 51446.7 & -1.1 & 51450.8 & 51470.6 & -19.8 & 2.6 \\
\hline 48 & 18 & 123917 & 738 & 110 & 299.33 & 51444.8 & 51445.9 & -1.1 & 51445.0 & 51464.8 & -19.8 & 0.1 \\
\hline 48 & 18 & 123926 & 740 & 110 & 304.33 & 51445.4 & 51446.5 & -1.1 & 51450.6 & 51470.4 & $\mid-19.8$ & 2.6 \\
\hline 48 & 18 & 123934 & 742 & 110 & 309.33 & 51455.2 & 51456.3 & -1.1 & 51450.8 & 51470.7 & -19.9 & -2.2 \\
\hline 48 & 18 & 123941 & 744 & 110 & 314.33 & 51454.2 & 51455.3 & -1.1 & 51453.2 & 51473.1 & -19.9 & -0.5 \\
\hline 49 & 18 & 125426 & 746 & 100 & 313.7 & 51457.4 & 51458.1 & -0.7 & 51460.2 & 51482.8 & -22.6 & 1.4 \\
\hline 49 & 18 & 125432 & 748 & 100 & 308.7 & 51455.6 & 51456.3 & -0.7 & 51455.0 & 51477.7 & -22.7 & -0.3 \\
\hline 49 & 18 & 125440 & 750 & 100 & 303.7 & 51456.8 & 51457.5 & -0.7 & 51450.8 & 73.5 & -22.7 & -3.0 \\
\hline 49 & 18 & 125447 & 752 & 100 & 298.7 & 51449.6 & 51450.3 & -0.7 & 51458.6 & 81.3 & -22.7 & 4.5 \\
\hline 49 & 18 & 125454 & 754 & 100 & 293.7 & 51449.2 & 51449.9 & -0.7 & 51456.6 & 51479.3 & -22.7 & 3.7 \\
\hline $49^{\circ}$ & 18 & 125501 & 756 & 100 & 288.7 & 51447.0 & 51447.7 & -0.7 & 51448.8 & 51471.6 & -22.8 & 0.9 \\
\hline 49 & 18 & 125508 & 758 & 100 & 283.7 & 51444.2 & 51444.9 & -0.7 & 51446.6 & 51469.4 & -22.8 & 1.2 \\
\hline 49 & 18 & 125515 & 760 & 100 & 278.7 & 51443.4 & 51444.1 & -0.7 & 51447.4 & 51470.2 & -22.8 & 2.0 \\
\hline 49 & 18 & 125524 & 762 & 100 & 273.7 & 51433.8 & 51434.5 & -0.7 & 51448.8 & 51471.6 & -22.8 & 7.5 \\
\hline 49 & 18 & 125532 & 764 & 100 & 268.7 & 51425.6 & 51426.3 & -0.7 & 51444.6 & 51467.4 & -22.8 & 9.5 \\
\hline 49 & 18 & 125541 & 766 & 100 & 263.7 & 51422.8 & 51423.5 & -0.7 & 51427.6 & 50.5 & -22.9 & 2.4 \\
\hline 49 & 18 & 12 & 768 & 100 & 258.7 & 514 & 10.7 & -0.7 & 22.0 & 44.9 & -22.9 & 6.0 \\
\hline 49 & 18 & 125558 & 770 & 100 & 253.7 . & 51391.8 & 51392.5 & -0.7 & 11.4 & 51434.3 & $\mid-22.9$ & 9.8 \\
\hline 49 & 18 & 125609 & 772 & 100 & 248.7 & 51351.0 & 51351.7 & -0.7 & 51324.4 & 51347.4 & -23.0 & -13.3 \\
\hline 49 & 18 & 125617 & 774 & 100 & 243.7 & 51341.8 & 51342.5 & -0.7 & 51451.0 & 51474.0 & -23.0 & 54.6 \\
\hline 49 & 18 & 125624 & 776 & 100 & 238.7 & 51284.6 & 51285.3 & -0.7 & 51309.4 & 51332.4 & $\mid-23.0$ & 12.4 \\
\hline 49 & 18 & 125632 & 778 & 100 & 233.7 & 51205.4 & 51206.1 & -0.7 & 51229.2 & 51252.2 & -23.0 & 11.9 \\
\hline 49 & 18 & 125639 & 780 & 100 & 228.7 & 51081.0 & 51081.7 & -0.7 & 51102.2 & 51125.3 & $\mid-23.1$ & 10.6 \\
\hline 49 & 18 & 125647 & 782 & 100 & 223.7 & 50895.4 & 50896.1 & -0.7 & 50887.6 & 50910.7 & $\mid-23.1$ & -3.9 \\
\hline 49 & 18 & 125654 & 784 & 100 & 218.7 & 50666.4 & 50667.0 & -0.6 & 50552.4 & 50575.5 & -23.1 & -57.0 \\
\hline 49 & 18 & 125701 & 786 & 100 & 213.7 & 50486.4 & 50487.0 & -0.6 & 50112.6 & 50135.7 & -23.1 & -186.9 \\
\hline 49 & 18 & 125708 & 788 & 100 & 208.7 & 50819.8 & $|50820.4|$ & -0.6 & 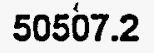 & 50530.4 & $\mid-23.2$ & -156.3 \\
\hline
\end{tabular}




\begin{tabular}{|c|c|c|c|c|c|c|c|c|c|c|c|c|}
\hline Line & Day & Time & Sta. & $x$ & $\mathbf{Y}$ & uncorr. & $\begin{array}{l}(\mathrm{nT}) \\
\text { corr. }\end{array}$ & diff. & $\begin{array}{l}\text { BOT } \\
\text { uncorr. }\end{array}$ & $\begin{array}{l}\text { OM }(n T) \\
\text { corr. }\end{array}$ & diff. & $\begin{array}{c}\text { Vert.Grad. } \\
\text { (nT/ft) }\end{array}$ \\
\hline 49 & 18 & 125716 & 790 & 100 & 203.7 & $5 \cdot 1630.0$ & 51630.6 & -0.6 & 52202.4 & 52225.6 & -23.2 & 286.2 \\
\hline 49 & 18 & 125724 & 792 & 100 & 198.7 & 106.0 & 52106.6 & -0.6 & 52891.0 & 52914.2 & -23.2 & 2.5 \\
\hline 49 & 18 & 125731 & 7.94 & 100 & 193.7 & 52206.6 & 52207.2 & -0.6 & 52865.8 & 52889.0 & -23.2 & 329.6 \\
\hline 49 & 18 & 125739 & 798 & 100 & 188.7 & 51951.2 & 51951.8 & -0.6 & 52247.4 & 52270.6 & $\mid-23.2$ & 148.1 \\
\hline 49 & 18 & 125746 & 798 & 100 & 183.7 & 51633.6 & 51634.2 & -0.6 & 51679.0 & 51702.3 & -23.3 & 22.7 \\
\hline 49 & 18 & 125753 & 800 & 100 & 178.7 & 51447.4 & 51448.0 & -0.6 & 51421.0 & 51444.3 & -23.3 & -13.2 \\
\hline 49 & 18 & 125759 & 802 & 100 & 173.7 & 51363.6 & 51364.2 & -0.6 & 51328.4 & 51351.7 & -23.3 & -17.6 \\
\hline 49 & 18 & 125806 & 804 & 100 & 168.7 & 51339.0 & 51339.6 & -0.6 & 51318.2 & 51341.5 & -23.3 & -10.4 \\
\hline 49 & 18 & 125813 & 806 & 100 & 163.7 & 51344.4 & 51345.0 & -0.6 & 51328.8 & 51352.2 & $|-23.4|$ & -7.8 \\
\hline 50 & 18 & 130309 & 818 & 90 & 163.07 & 51334.6 & 51334.6 & 0.0 & 51323.8 & 51346.0 & -22.2 & -5.4 \\
\hline 50 & 18 & 130317 & 820 & 90 & 168.07 & 51326.4 & 51326.3 & 0.1 & 51305.0 & 51327.3 & -22.3 & -10.7 \\
\hline 50 & 18 & 130324 & 822 & 90 & 173.07 & 51325.8 & 51325.7 & 0.1 & 51291.2 & 51313.5 & -22.3 & -17.3 \\
\hline 50 & 18 & 130331 & 824 & 90 & 178.07 & 51363.2 & 51363.1 & 0.1 & 51304.8 & 51327.0 & -22.2 & -29.2 \\
\hline 50 & 18 & 130337 & 826 & 90 & 183.07 & 51465.2 & 51465.1 & 0.1 & 51420.8 & 51443.0 & -22.2 & -22.2 \\
\hline 50 & 18 & 130344 & 828 & 90 & 188.07 & 51674.4 & 51674.3 & 0.1 & .51703 .6 & 51725.8 & -22.2 & 14.6 \\
\hline 50 & 18 & 130350 & 830 & 90 & 193.07 & 51759.2 & 51759.0 & 0.2 . & 51865.2 & 51887.3 & -22.1 & 53.0 \\
\hline 50 & 18 & 130357 & 832 & 90 & 198.07 & 51477.8 & 51477.6 & 0.2 & 51323.2 & 51345.3 & $\mid-22.1$ & -77.3 \\
\hline 50 & 18 & 130405 & 834 & 90 & 203.07 & 50961.6 & 50961.4 & 0.2 & 50413.8 & 50435.9 & -22.1 & -273.9 \\
\hline 50 & 18 & 130413 & 836 & 90 & 208.07 & 50703.6 & 50703.4 & 0.2 & 50604.2 & 50626.2 & -22.0 & -49.7 \\
\hline 50 & 18 & 130420 & 838 & 90 & 213.07 & 50544.0 & 50543.8 & 0.2 & 50194.0 & 50216.0 & -22.0 & -175.0 \\
\hline 50 & 18 & 130426 & 840 & 90 & 218.07 & 50743.6 & 50743.3 & 0.3 & 50647.4 & 50669.4 & -22.0 & -48.1 \\
\hline 50 & 18 & 130433 & 842 & 90 & 223.07 & 50984.4 & 50984.1 & 0.3 & 50972.2 & 50994.1 & -21.9 & -6.1 \\
\hline 50 & 18 & 130440 & 844 & 90 & 228.07 & 51159.2 & |158.9 & 0.3 & 51148.8 & 51170.7 & -21.9 & -5.2 \\
\hline 50 & 18 & 130446 & 846 & 90 & 233.07 & 51255.2 & 1254.9 & 0.3 & 51270.4 & 51292.3 & -21.9 & 7.6 \\
\hline 50 & 18 & 130454 & 848 & 90 & 238.07 & 51316.0 & 315.7 & 0.3 & 51337.8 & 1359.6 & -21.8 & 10.9 \\
\hline 50 & 18 & 130501 & 850 & 90 & 243.07 & 51350.8 & $\mid 350.4$ & 0.4 & 51376.4 & 51398.2 & -21.8 & 12.8 \\
\hline 50 & 18 & 130513 & 852 & 90 & 248.07 & 51388.2 & 51387.8 & 0.4 & ,51411.4 & 51433.1 & -21.7 & 11.6 \\
\hline 50 & 18 & 130520 & 854 & 90 & 253.07 & 51407.2 & 51406.8 & 0.4 & 51430.0 & 51451.7 & -21.7 & 11.4 \\
\hline 50 & 18 & 130528 & 856 & 90 & 258.07 & 51421.0 & 51420.6 & 0.4 & 51429.4 & 51451.1 & -21.7 & 4.2 \\
\hline 50 & 18 & 130536 & 858 & 90 & 263.07 & 51436.2 & 51435.8 & 0.4 & 51435.4 & 51457.0 & -21.6 & -0.4 \\
\hline 50 & 18 & 130543 & 860 & 90 & 268.07 & 51441.4 & 51440.9 & 0.5 & 51437.8 & 51459.4 & -21.6 & -1.8 \\
\hline 50 & 18 & 130550 & 862 & 90 & 273.07 & 51446.8 & 51446.3 & 0.5 & 51443.6 & 51465.2 & $\mid-21.6$ & -1.6 \\
\hline 50 & 18 & 130558 & 864 & 90 & 278.07 & 51450.4 & 51449.9 & 0.5 & 51448.2 & 51469.7 & -21.5 & -1.1 \\
\hline 50 & 18 & 130605 & 866 & 90 & 283.07 & 51451.2 & 450.7 & 0.5 & 51447.4 & 51468.9 & -21.5 & -1.9 \\
\hline 50 & 18 & 130613 & 868 & 90 & 288.07 & 51456.2 & 455.7 & 0.5 & 51452.6 & 51474.1 & -21.5 & -1.8 \\
\hline 50 & 18 & 130622 & 870 & 90 & 293.07 & 51458.6 & 458.0 & 0.6 & 51454.8 & 51476.2 & -21.4 & -1.9 \\
\hline 50 & 18 & 130630 & 872 & 90 & 298.07 & 51460.0 & 51459.4 & 0.6 & 157.6 & 51479.0 & -21.4 & -1.2 \\
\hline 51 & 18 & 30931 & 874 & 80 & 297.44 & 51460.2 & 459.1 & 1.1 & 51475.2 & 51495.7 & -20.5 & 7.5 \\
\hline 51 & 18 & 30938 & 876 & 80 & 292.44 & 51457.2 & 51456.1 & 1.1 & 51468.4 & 51488.9 & -20.5 & 5.6 \\
\hline 51 & 18 & 130946 & 878 & 80 & 287.44 & 51453.2 & 51452.1 & 1.1 & 51467.2 & 51487.7 & -20.5 & 7.0 \\
\hline 51 & 18 & 130954 & 880 & 80 & 282.44 & 51455.6 & 51454.5 & 1.1 & $51,465.4$ & 51485.8 & -20.4 & 4.9 \\
\hline 51 & 18 & 131002 & 882 & 80 & 277.44 & 51452.8 & 451.7 & 1.1 & 51460.2 & 51480.6 & -20.4 & 3.7 \\
\hline 51 & 18 & 131009 & 884 & 80 & 272.44 & 51448.0 & 51446.8 & 1.2 & 51456.8 & 51477.1 & -20.3 & 4.4 \\
\hline 51 & 18 & 31015 & 886 & 80 & 267.44 & 51443.6 & 51442.4 & 1.2 & 51452.0 & 51472.3 & -20.3 & 4.2 \\
\hline 5 & 18 & 131023 & 888 & 80 & 262.44 & 51437.8 & 51436.6 & 1.2 & 51454.2 & 51474.5 & -20.3 & 8.2 \\
\hline 51 & 18 & 131030 & 890 & 80 & 257.44 & 51430.8 & 51429.6 & 1.2 & 51446.6 & 51466.8 & -20.2 & 7.9 \\
\hline 51 & 18 & 131037 & 892 & 80 & 252.44 & 51432.8 & 51431.6 & 1.2 & 51427.2 & 51447.4 & -20.2 & -2.8 \\
\hline 51 & 18. & 131045 & 894 & 80 & 247.44 & 51412.6 & 51411.3 & 1.3 & 51420.0 & 51440.2 & -20.2 & 3.7 \\
\hline 51 & 18 & 131053| & 896 & 80 & 242.44 & 51391.0 & 51389.7 & 1.3 & 51404.4 & 51424.5 & & 6.7 \\
\hline
\end{tabular}




\begin{tabular}{|c|c|c|c|c|c|c|c|c|c|c|c|c|}
\hline ne & & imo & & & & & & & $\mathrm{BC}$ & $\mathrm{nT}$ & diff & Vert.Grad. \\
\hline 51 & 18 & 131114 & 898 & 80 & 237.44 & 51361.4 & 51360.1 & 1.3 & 51384.4 & 51404.4 & -20.0 & 11.5 \\
\hline 51 & 18 & 131128 & 900 & 80 & 232.44 & 51312.8 & 51311.4 & 1.4 & 51344.4 & 51364.4 & -20.0 & 15.8 \\
\hline 51 & 18 & 131141 & 902 & 80 & 227.44 & 51202.4 & 51201.0 & 1.4 & 51237.6 & 51257.5 & -19.9 & 17.6 \\
\hline 51 & 18 & 131149 & 904 & 80 & 222.44 & 51060.6 & 51059.2 & 1.4 & 51084.4 & 51104.3 & -19.9 & 11.9 \\
\hline 51 & 18 & 131156 & 906 & 80 & 217.44 & 50788.0 & 50786.6 & 1.4 & 50736.8 & 50756.6 & -19.8 & -25.6 \\
\hline 51 & 18 & 131204 & 908 & 80 & 212.44 & 50404.2 & 50402.7 & 1.5 & 50033.0 & 50052.8 & -19.8 & -185.6 \\
\hline 51 & 18 & 131212 & 910 & 80 & 207.44 & 50310.2 & 50308.7 & 1.5 & 49597.6 & 49617.4 & -19.8 & -356.3 \\
\hline 51 & 18 & 131220 & .912 & 80 & 202.44 & 51252.8 & 51251.3 & 1.5 & 51526.4 & 51546.1 & $\mid-19.7$ & 136.8 \\
\hline 51 & 18 & 131228 & 914 & 80 & 197.44 & 51861.2 & 51859.7 & 1.5 & 52994.0 & 53013.7 & -19.7 & 566.4 \\
\hline 51 & 18 & 131235 & 916 & 80 & 192.44 & 51677.8 & 51676.3 & 1.5 & 51825.2 & 51844.9 & -19.7 & 73.7 \\
\hline 51 & 18 & 131244 & 918 & 80 & 187.44 & 51387.6 & 51386.0 & 1.6 & 51381.0 & 51400.6 & $\mid-19.6$ & -3.3 \\
\hline 51 & 18 & 131251 & 920 & 80 & 182.44 & 51271.4 & 51269.8 & 1.6 & 51202.6 & 51222.2 & -19.6 & -34.4 \\
\hline 51 & 18 & 131258 & 922 & 80 & 177.44 & 51256.0 & 254.4 & 1.6 & 13.6 & 51233.2 & -19.6 & -21.2 \\
\hline 51 & 18 & 131306 & 924 & 80 & 172.44 & 51285.4 & 51283.8 & 1.6 & 67.8 & 51287.3 & -19.5 & -8.8 \\
\hline 51 & 18 & 131313 & 926 & 80 & 167.44 & 51319.6 & 51318.0 & 1.6 & 51310.0 & 51329.5 & $\mid-19.5$ & -4.8 \\
\hline 51 & 18 & 131321 & 928 & 80 & 162.44 & 51336.8 & 51335.1 & 1.7 & 51328.4 & 51347.8 & -19.4 & -4.2 \\
\hline 52 & 18 & 131604 & 930 & 70 & 161.81 & 51333.2 & 331.1 & 2.1 & 36.0 & $|354.7|$ & -18.7 & 1.4 \\
\hline 52 & 18 & 13 & 932 & 70 & 16 & & 07.1 & 2.1 & 8.8 & 17.4 & -18.6 & -5.2 \\
\hline 52 & 18 & 131620 & 934 & 70 & 171.81 & 51251.0 & 51248.9 & 2.1 & 10.2 & 51128.8 & -18.6 & -70.4 \\
\hline 52 & 18 & 131628 & 936 & 70 & 176.81 & 51229.6 & 51227.4 & 2.2 & 193.0 & 51211.6 & -18.6 & -18.3 \\
\hline 52 & 18 & 131636 & 938 & 70 & 181.81 & 51187.4 & 51185.2 & 2.2 & 51122.6 & 51141.1 & -18.5 & -32.4 \\
\hline 52 & 18 & 131644 & 940 & 70 & 186.81 & 51140.8 & 51138.6 & 2.2 & 50985.8 & 51004.3 & -18.5 & -77.5 \\
\hline 52 & 18 & 131652 & 942 & 70 & 191.81 & 51159.0 & 51156.8 & 2.2 & 50973.2 & 50991.6 & -18.4 & -92.9 \\
\hline 52 & 18 & 131701 & 944 & 70 & 196.81 & 51052.2 & 51050.0 & 2.2 & 50919.6 & 50938.0 & -18.4 & -66.3 \\
\hline 52 & 18 & 131709 & 946 & 70 & 201.81 & 50756.4 & 50754.1 & 2.3 & 50326.0 & 50344.4 & -18.4 & -215.2 \\
\hline 52 & 18 & 131717 & 948 & 70 & 206.81 & 50725.6 & 723.3 & 2.3 & 33.6 & 0601.9 & -18.3 & -71.0 \\
\hline 52 & 18 & 131725 & 950 & 70 & 211.81 & 5.6 & 853.3 & 2.3 & 50828.0 & 0846.3 & $\mid-18.3$ & -13.8 \\
\hline 52 & 18 & 131733 & 952 & 70 & 216.81 & 51044.4 & 042.1 & 2.3 & 51053.2 & 1071.5 & $|-18.3|$ & 4.4 \\
\hline 52 & 18 & 131740 & 954 & 70 & 221.81 & 51204.2 & 51201.8 & 2.4 & 51237.2 & 51255.4 & -18.2 & 16.5 \\
\hline 52 & 18 & 131748 & 956 & 70 & 226.81 & 51301.4 & 51299.0 & 2.4 & 36.4 & 51354.6 & $|-18.2|$ & 17.5 \\
\hline 52 & 18 & 131756 & 958 & 70 & 231.81 & 51362.4 & 51360.0 & 2.4 & 80.4 & 51398.5 & -18.1 & 9.0 \\
\hline 52 & 18 & 131804 & 960 & 70 & 236.81 & 51397.6 & 51395.2 & 2.4 & 51416.0 & 51434.1 & -18.1 & 9.2 \\
\hline 52 & 18 & 131812 & 962 & $\cdot 70$ & 241.81 & 51424.0 & 51421.6 & 2.4 & 51451.0 & 51469.1 & -18.1 & 13.5 \\
\hline 52 & 18 & 131821 & 964 & 70 & 246.81 & 51439.6 & 51437.1 & 2.5 & 51452.6 & 51470.6 & -18.0 & 6.5 \\
\hline 52 & 18 & 131828 & 966 & 70 & 251.81 & 51446.4 & 51443.9 & 2.5 & 1455.0 & 1473.0 & -18.0 & 4.3 \\
\hline 52 & 18 & 37 & 968 & 70 & 256.81 & 51452.0 & 51449.5 & 2.5 & 60.2 & 51478.2 & -18.0 & 4.1 \\
\hline 52 & 18 & 131845 & 970 & 70 & 261.81 & 51456.6 & 51454.1 & 2.5 & 51460.0 & 51477.9 & -17.9 & 1.7 \\
\hline 52 & 18 & 131859 & 972 & 70 & 266.81 & 51460.6 & 51458.0 & 2.6 & 51460.0 & 51477.9 & -17.9 & -0.3 \\
\hline 52 & 18 & 131907 & 974. & 70 & 271.81 & 51463.4 & 51460.8 & 2.6 & 51459.8 & 51477.6 & -17.8 & -1.8 \\
\hline 52 & 18 & 131915 & 976 & 70 & 276.81 & 51463.6 & 51461.0 & 2.6 & 466.0 & 51483.8 & -17.8 & 1.2 \\
\hline 52 & 18 & 131923 & 978 & 70 & 281.81 & 51460.2 & 51457.6 & 2.6 & 51477.2 & 51494.9 & -17.7 & 8.5 \\
\hline 52 & 18 & 131935 & 980 & 70 & 286.81 & 51462.8 & 51460.1 & 2.7 & 51472.2 & 51489.9 & -17.7 & 4.7 \\
\hline 53 & 18 & 132403 & 982 & 60 & 296.18 & 51463.8 & 51460.4 & 3.4 & 51479.8 & 51496.2 & -16.4 & 8.0 \\
\hline 53 & 18 & 132411 & 984 & 60 & 291.18 & 51463.6 & 51460.2 & 3.4 & 51475.8 & 51492.2 & -16.4 & 6.1 \\
\hline 53 & 18 & 132420 & 986 & 60 & 286.18 & 51466.0 & 462.6 & 3.4 & 51487.8 & 1504.1 & -16.3 & 10.9 \\
\hline 53 & .18 & 132428 & 988 & 60 & 281.18 & 51465.2 & 51461.8 & 3.4 & 51476.2 & 51492.5 & $|-16.3|$ & 5.5 \\
\hline 53 & 18 & 132436 & 990 & 60 & 276.18 & $51464.8^{\circ}$ & 51461.4 & 3.4 & 51472.6 & 51488.9 & -16.3 & 3.9 \\
\hline 53 & 18 & 132445 & 992 & 60 & 271.18 & 51461.4 & 51457.9 & 3.5 & 51470.6 & 51486.8 & -16.2 & 4.6 \\
\hline 53 & 18 & 132453 & 994 & 60 & 266.18 & 1459.6 & 51456.1 & 3.5 & 51467.4 & 51483.6 & $|-16.2|$ & .9 \\
\hline
\end{tabular}




\begin{tabular}{|c|c|c|c|c|c|c|c|c|c|c|c|c|}
\hline & & & & & & & $\mathrm{JP}(\mathrm{nT})$ & & $\mathrm{BO}^{7}$ & TOM (nT & & \\
\hline Line & Day & Time & Sta. & $\mathbf{x}$ & $\mathbf{Y}$ & uncorr. & corr. & diff. & uncorr. & corr. & diff. & \\
\hline 53 & 18 & 132502 & 996 & 60 & 261.18 & 51455.8 & 51452.3 & 3.5 & 51465.4 & 51481.5 & -16.1 & 4.8 \\
\hline 53 & 18 & 132511 & 998 & 60 & 256.18 & 51453.8 & 51450.3 & 3.5 & 51470.8 & 51486.9 & -16.1 & 8.5 \\
\hline 53 & 18 & 132518 & 1000 & 60 & 251.18 & $5 i 454.0$ & 51450.4 & 3.6 & 51457.8 & 51473.9 & -16.1 & 1.9 \\
\hline 53 & 18 & 132525 & 1002 & 60 & 246.18 & 51445.8 & 51442.2 & 3.6 & 51457.0 & 51473.0 & -16.0 & 5.6 \\
\hline 53 & 18 & 132534 & 1004 & 60 & 241.18 & 51437.4 & 51433.8 & 3.6 & 51455.2 & 51471.2 & -16.0 & 8.9 \\
\hline 53 & 18 & 132543 & 1006 & 60 & 236.18 & 51422.8 & 51419.2 & 3.6 & 51447.6 & 51463.5 & $|-15.9|$ & 12.4 \\
\hline 53 & 18 & 132551 & 1008 & 60. & 231.18 & 51402.6 & 51399.0 & 3.6 & 51432.0 & 51447.9 & -15.9 & 14.7 \\
\hline 53 & 18 & 132559 & 1010 & 60 & 226.18 & 51361.8 & 51358.1 & 3.7 & 51403.0 & 51418.9 & -15.9 & 20.6 \\
\hline 53 & 18 & 132606 & 1012 & 60 & 221.18 & 51287.6 & 51283.9 & 3.7 & 51337.6 & 51353.4 & -15.8 & 25.0 \\
\hline 53 & 18 & 132614 & 1014 & 60 & 216.18 & 51100.0 & 51096.3 & 3.7 & 51152.6 & 51168.4 & $\mid-15.8$ & 26.3 \\
\hline 53 & 18 & 132622 & 1016 & 60 & 211.18 & 50715.4 & 50711.7 & 3.7 & 50327.0 & 50342.8 & -15.8 & -194.2 \\
\hline 53 & 18 & 132630 & 1018 & 60 & 206.18 & 50727.0 & 50723.3 & 3.7 & 50767.0 & 50782.7 & $|-15.7|$ & 20.0 \\
\hline 53 & 18 & 132638 & 1020 & 60 & 201.18 & 50521.4 & 50517.6 & 3.8 & 50063.8 & 50079.5 & -15.7 & -228.8 \\
\hline 53 & 18 & 132645 & 1022 & 60 & 196.18 & 50857.0 & 50853.2 & 3.8 & 50800.4 & 50816.1 & -15.7 & -28.3 \\
\hline 53 & 18 & 132653 & 1024 & 60 & 191.18 & 51256.4 & 51252.6 & 3.8 & 51530.2 & 51545.8 & -15.6 & 136.9 \\
\hline 53 & 18 & 132701 & 1026 & 60 & 186.18 & 51277.8 & 51274.0 & 3.8 & 51341.4 & 51357.0 & $|-15.6|$ & 31.8 \\
\hline 53 & 18 & 132709 & 1028 & 60 & 181.18 & 51271.6 & 51267.8 & 3.8 & 51276.0 & 51291.5 & $|-15.5|$ & 2.2 \\
\hline 53 & 18 & 132717 & 1030 & 60 & 176.18 & 51286.2 & 51282.3 & 3.9 & 51284.4 & 51299.9 & $|-15.5|$ & -0.9 \\
\hline 53 & 18 & 132720 & 1032 & 60 & 171.18 & $\cdot 51311.8$ & 51307.9 & 3.9 & 51311.8 & 51327.3 & $\mid-15.5$ & 0.0 \\
\hline 53 & 18 & 132733 & 1034 & 60 & 166.18 & 51339.6 & 51335.7 & 3.9 & 51328.0 & 51343.4 & $|-15.4|$ & -5.8 \\
\hline 53 & 18 & 132741 & 1036 & 60 & 161.18 & 51360.8 & 51356.9 & 3.9 & 51351.6 & 51367.0 & $|-15.4|$ & -4.6 \\
\hline 54 & 18 & 133410 & 1048 & 50 & 160.55 & 51373.0 & 51368.5 & $4: 5$ & 51379.8 & 51396.2 & $|-16.4|$ & 3.4 \\
\hline 54 & 18 & 133419 & 1050 & 50 & 165.55 & 51360.2 & 51355.5 & 4.7 & 51345.4 & 51361.6 & -16.2 & -7.4 \\
\hline 54 & 18 & 133427 & 1052 & 50 & 170.55 & 51339.4 & 51334.7 & 4.7 & 4.6 & 51330.8 & -16.2 & -12.4 \\
\hline 54 & 18 & 133435 & 1054 & 50 & 175.55 & 51317.2 & 51312.5 & 4.7 & 51262.4 & 51278.6 & -16.2 & -27.4 \\
\hline 54 & 18 & 133444 & 1056 & 50 & 180.55 & 51292.0 & 51287.3 & 4.7 & 51213.6 & 51229.9 & -16.3 & -39.2 \\
\hline 54 & 18 & 133451 & 1058 & 50 & 185.55 & 51222.0 & 51217.3 & 4.7 & 51145.4 & 51161.7 & -16.3 & -38.3 \\
\hline 54 & 18 & 133459 & 1060 & 50 , & 190.55 & 51087.4 & 51082.7 & 4.7 & 51038.2 & 51054.5 & -16.3 & -24.6 \\
\hline 54 & 18 & 133509 & 1062 & 50 & 195.55 & 1.2 & 6.5 & 4.7 & 7.2 & 50733.5 & -16.3 & -62.0 \\
\hline 54 & 18 & 133517 & 1064 & 50 & 200.55 & 50696.6 & 50691.8 & 4.8 & 50469.8 & 50486.2 & -16.4 & -113.4 \\
\hline 54 & 18 & 133525 & 1066 & 50 & 205.55 & 50970.0 & 50965.2 & 4.8 & 51018.0 & 51034.4 & $\mid-16.4$ & 24.0 \\
\hline 54 & 18 & 133534 & 1068 & 50 & 210.55 & 51187.8 & 51183.0 & 4.8 & 51265.4 & 51281.8 & -16.4 & 38.8 \\
\hline 54 & 18 & 133542 & 1070 & 50 & 215.55 & 51304.0 & 51299.2 & 4.8 & 51345.4 & 51361.9 & -16.5 & 20.7 \\
\hline 54 & 18 & 133549 & 1072 & 50 & 220.55 & 51368.4 & 51363.6 & 4.8 & 51389.4 & 51405.9 & $\mid-16.5$ & 10.5 \\
\hline 54 & 18 & 133558 & 1074 & 50 & 225.55 & 51400.2 & 51395.4 & 4.8 & 16.2 & 51432.7 & $\mid-16.5$ & 8.0 \\
\hline 54 & 18 & 133607 & 1076 & 50 & 230.55 & 51417.0 & 51412.2 & 4.8 & 2.2 & 51408.7 & -16.5 & -12.4 \\
\hline 55 & 18 & 134047 & 1078 & 40 & 239.92 & 51447.6 & 51442.3 & 5.3 & 56.2 & 51473.6 & -17.4 & 4.3 \\
\hline 55 & 18 & 134056 & 1080 & 40 & 234.92 & 51431.8 & 51426.5 & 5.3 & 51450.4 & 51467.9 & -17.5 & 9.3 \\
\hline 55 & 18 & 134104 & 1082 & 40 & 229.92 & 51403.4 & 51398.1 & 5.3 & 51417.0 & 51434.5 & -17.5 & 6.8 \\
\hline 55 & 18 & 134112 & 1084 & 40 & 224.92 & 51327.4 & 51322.1 & 5.3 & 51308.2 & 51325.7 & -17.5 & -9.6 \\
\hline 55 & 18 & 134121 & 1086 & 40 & 219.92 & 51238.8 & 51233.4 & 5.4 & 50856.4 & 50874.0 & $|-17.6|$ & -191.2 \\
\hline 55 & 18 & 134130 & 1088 & 40 & 214.92 & 51740.4 & 51735.0 & 5.4 & 52010.2 & 52027.8 & $|-17.6|$ & 134.9 \\
\hline 55 & 18 & 134139 & 1090 & 40 & 209.92 & 51616.6 & 51611.2 & 5.4 & 51128.4 & 51146.0 & $\mid-17.6$ & -244.1 \\
\hline 55 & 18 & 134147 & 1092 & 40 & 204.92 & 51815.2 & 51809.8 & 5.4 & 58619.2 & 58636.8 & -17.6 & 3402.0 \\
\hline 55 & 18 & 134155 & 1094 & 40 & 199.92 & 51422.2 & 51416.8 & 5.4 & 51380.2 & 51397.9 & -17.7 & -21.0 \\
\hline 55 & 18 & 134203 & 1096 & 40 & 194.92 & 51246.6 & 51241.2 & 5.4 & 51239.0 & 51256.7 & $\mid-17.7$ & -3.8 \\
\hline 55 & 18 & 134210 & 1098 & 40 & 189.92 & 51097.4 & 51092.0 & 5.4 & 50983.2 & 51000.9 & -17.7 & -57.1 \\
\hline 55 & 18 & 134219 & 1100 & 40 & 184.92 & 50875.6 & 50870.1 & 5.5 & 49487.8 & 49505.5 & -17.7 & -693.9 \\
\hline 55 & 18 & 134227 & 1102 & 40 & 179.92 & 58594.2 & 58588.7 & 5.5 & 58612.2 & 58630.0 & -17.8 & 9.0 \\
\hline
\end{tabular}




\begin{tabular}{|c|c|c|c|c|c|c|c|c|c|c|c|c|}
\hline & & & & & & & (T) & & $\mathbf{B C}$ & (1) & & ent. \\
\hline Line & Day & Time & Sta. & $x$ & $\mathbf{Y}$ & uncort. & corr. & diff. & uncorr. & corr. & diff. & \\
\hline 55 & 18 & 134234 & 1104 & 40 & 174.92 & 51390.8 & 51385.3 & 5.5 & 50745.8 & 50763.6 & -17.8 & -322.5 \\
\hline 55 & 18 & 134242 & 1106 & 40 & 169.92 & 51334.8 & 51329.3 & 5.5 & 51233.0 & 51250.8 & -17.8 & -50.9 \\
\hline 55 & 18 & 134249 & 1108 & 40 & 164.92 & 51362.2 & 51356.7 & 5.5 & 51344.2 & 51362.0 & -17.8 & -9.0 \\
\hline 55 & 18 & 134256 & 1110 & 40 & 159.92 & 51383.4 & 51377.9 & 5.5 & 51386.8 & 51404.7 & -17.9 & 1.7 \\
\hline 56 & 18 & 134423 & 1112 & 30 & 159.29 & 51394.8 & 51389.1 & 5.7 & 51407.2 & 51425.3 & -18.1 & 6.2 \\
\hline 56 & 18 & 134431 & 1114 & 30 & 164.29 & 51376.6 & 51370.9 & 5.7 & 51358.6 & 51376.8 & -18.2 & -9.0 \\
\hline 56 & 18 & 134441 & 1116 & 30 & 169.29 & 51332.6 & 51326.9 & 5.7 & 51237.0 & 51255.2 & -18.2 & -47.8 \\
\hline 56 & 18 & 134450 & 1118 & 30 & 174.29 & 51185.2 & 51179.5 & 5.7 & 50967.2 & 50985.4 & -18.2 & -109.0 \\
\hline 56 & 18 & 134458 & 1120 & 30 & 179.29 & 51015.0 & 51009.3 & 5.7 & 50883.2 & 50901.4 & -18.2 & -65.9 \\
\hline 56 & 18 & 134506 & 1122 & 30 & 184.29 & 51044.8 & 51039.1 & 5.7 & 50986.8 & 51005.1 & -18.3 & -29.0 \\
\hline 56 & 18 & 134513 & 1124 & 30 & 189.29 & 51217.0 & 51211.3 & 5.7 & 51206.6 & 51224.9 & -18.3 & -5.2 \\
\hline 56 & 18 & 134520 & 1126 & 30 & 194.29 & 51319.2 & 51313.4 & 5.8 & 51310.8 & 51329.1 & $\mid-18.3$ & -4.2 \\
\hline 56 & 18 & 134527 & 1128 & 30 & 199.29 & 51350.2 & 1344.4 & 5.8 & 51320.6 & 51338.9 & -18.3 & -14.8 \\
\hline 56 & 18 & 134537 & 1130 & 30 & 204.29 & 51307.0 & 51301.2 & 5.8 & 51202.4 & 51220.8 & $\mid-18.4$ & -52.3 \\
\hline 56 & 18 & 134545 & 1132 & 30 & 209.29 & 51155.8 & 51150.0 & 5.8 & 50808.8 & 50827.2 & -18.4 & -173.5 \\
\hline 56 & 18 & 134552 & 1134 & 30 & 214.29 & 50616.8 & 50611.0 & 5.8 & 49112.4 & 49130.8 & $\mid-18.4$ & -752.2 \\
\hline 56 & 18 & 134600 & 1136 & 30 & 219.29 & 50853.0 & 50847.2 & 5.8 & 50601.8 & .50620 .2 & $\mid-18.4$ & -125.6 \\
\hline 56 & 18 & 134608 & 1138 & 30 & 224.29 & 51278.2 & 51272.4 & 5.8 & 51283.2 & 51301.7 & -18.5 & 2.5 \\
\hline 56 & 18 & 134615 & 1140 & 30 & 229.29 & 51403.2 & 51397.4 & 5.8 & 51404.2 & 51422.7 & -18.5 & 0.5 \\
\hline 57 & 18 & 134907 & 1142 & 20 & 233.66 & 51454.6 & 51448.5 & 6.1 & 51466.2 & 51485.3 & $\mid-19.1$ & 5.8 \\
\hline 57 & 18 & 134914 & 1144 & 20 & 228.66 & 2.0 & 435.9 & 6.1 & 51459.4 & 51478.5 & $\mid-19.1$ & 8.7 \\
\hline 57 & 18 & 134922 & 1146 & 20 & 223.66 & 51425.0 & 418.8 & 6.2 & 51444.0 & 63.1 & $\mid-19.1$ & 9.5 \\
\hline 57 & 18 & 134930 & 1148 & 20 & 218.66 & 51425.4 & 51419.2 & 6.2 & 51459.4 & 51478.5 & -19.1 & 17.0 \\
\hline 57 & 18 & 134937 & 1150 & 20 & 213.66 & 51412.4 & 51406.2 & 6.2 & 51479.2 & 51498.4 & -19.2 & 33.4 \\
\hline 57 & 18 & 134944 & 1152 & 20 & 208.66 & 51402.8 & 51396.6 & 6.2 & 51417.0 & 51436.2 & -19.2 & 7.1 \\
\hline 57 & 18 & 134951 & 1154 & 20 & 203.66 & 51406.0 & 51399.8 & 6.2 & 51410.0 & 51429.2 & -19.2 & 2.0 \\
\hline 57 & 18 & 134958 & 1156 & 20 & 198.66 & 51415.0 & 51408.8 & 6.2 & 51436.4 & 51455.6 & -19.2 & 10.7 \\
\hline 57 & 18 & 135005 & 1158 & 20 & 193.66 & 51406.6 & 51400.4 & 6.2 & 51425.6 & 51444.8 & -19.2 & 9.5 \\
\hline 57 & 18 & 135011 & 1160 & 20 & 188.66 & 51394.4 & 51388.2 & 6.2 & 51413.2 & 51432.5 & -19.3 & 9.4 \\
\hline 57 & 18 & 135018 & 1162 & 20 & 183.66 & 51392.2 & 51385.9 & 6.3 & 51385.0 & 51404.3 & $\mid-19.3$ & -3.6 \\
\hline 57 & 18 & 135025 & 1164 & 20 & 178.66 & 51389.8 & 51383.5 & 6.3 & 51384.4 & 51403.7 & -19.3 & -2.7 \\
\hline 57 & 18 & 135032 & 1166 & 20 & 173.66 & 0.0 & 51393.7 & 6.3 & $51413.0^{\circ}$ & 51432.3 & $\mid-19.3$ & 6.5 \\
\hline 57 & 18 & 135039 & 1168 & 20 & 168.66 & 51413.0 & 51406.7 & 6.3 & 51410.6 & 51430.0 & $\mid-19.4$ & -1.2 \\
\hline 57 & 18 & 135046 & 1170 & 20 & 163.66 & 51418.6 & 51412.3 & 6.3 & 51422.0 & 51441.4 & $\mid-19.4$ & 1.7 \\
\hline 57 & 18 & 135053 & 1.172 & 20 & 158.66 & 51424.4 & 51418.1 & 6.3 & 51425.6 & 51445.0 & $\mid-19.4$ & 0.6 \\
\hline 58 & 18 & 135408 & 1174 & 10 & 158.03 & 51446.2 & 51439.6 & 6.6 & 51454.4 & 51474.4 & -20.0 & 4.1 \\
\hline 58 & 18 & 135416 & 1176 & 10 & 163.03 & 51459.6 & 51453.0 & 6.6 & 51466.8 & 51486.9 & $\mid-20.1$ & 3.6 \\
\hline 58 & 18 & 135424 & 1178 & 10 & 168.03 & 51458.2 & 51451.5 & 6.7 & 51476.2 & 51496.3 & $\mid-20.1$ & 9.0 \\
\hline 58 & 18 & 431 & 1180 & 10 & 173.03 & 514 & 128.9 & 6.7 & 51428.8 & 51448.9 & $\mid-20.1$ & -3.4 \\
\hline 58 & 18 & 135438 & 1182 & 10 & 178.03 & 51430.6 & 51423.9 & 6.7 & 51424.8 & 51444.9 & $\mid-20.1$ & -2.9 \\
\hline 58 & 18 & 135445 & 1184 & 10 & 183.03 & 51432.2 & 51425.5 & 6.7 & 51433.8 & 51454.0 & $\mid-20.2$ & 0.8 \\
\hline 58 & 18 & 135453 & 1186 & 10 & 188.03 & 51441.2 & 51434.5 & 6.7 & 51457.0 & 51477.2 & $\mid-20.2$ & 7.9 \\
\hline 58 & 18 & 135501 & 1188 & 10 & 193.03 & 51444.6 & 51437.9 & 6.7 & 51448.2 & 51468.4 & $\mid-20.2$ & 1.8 \\
\hline 58 & 18 & 135508 & 1190 & 10 & 198.03 & 51425.0 & 51418.3 & 6.7. & 51432.2 & 51452.4 & $\mid-20.2$ & 3.6 \\
\hline 58 & 18 & 135517 & 1192 & 10 & 203.03 & 51428.4 & 51421.6 & 6.8 & 52159.8 & 52180.1 & $\mid-20.3$ & 365.7 \\
\hline 58 & 18 & 135525 & $11 \mathrm{~s}$ & 10 & 208,03 & 51443.2 & 51436.4 & 6.8 & 51440.0 & 51460.3 & $\mid-20.3$ & -1.6 \\
\hline & 18 & 135533 & 1196 & 10 & 213.03 & 51437.8 & 51431.0 & 6.8 & 51441.6 & 51461.9 & $\mid-20.3$ & 1.9 \\
\hline 58 & 18 & 135541 & 1198 & 10 & 218.03 & 51443.4 & 51436.6 & 6.8 & 51453.8 & 51474.1 & -20.3 & 5.2 \\
\hline 58 & .18 & 135548 & 1200 & 10 & 223.03 & 51452.2 & 51445.4 & 6.8 & 51457.0 & 51477.4 & $\mid-20.4$ & -2.4 \\
\hline
\end{tabular}




\begin{tabular}{|c|c|c|c|c|c|c|c|c|c|c|c|c|}
\hline \multirow{2}{*}{ Line } & \multirow[b]{2}{*}{ Day } & \multirow[b]{2}{*}{ Time } & \multirow[b]{2}{*}{ | Sta. } & \multirow[b]{2}{*}{$\mathbf{x}$} & \multirow[b]{2}{*}{$\mathbf{Y}$} & \multicolumn{3}{|c|}{ TOP (nT) } & \multicolumn{2}{|c|}{ BOTTOM (nT) } & \multirow[b]{2}{*}{ diff. } & \multirow{2}{*}{$\begin{array}{c}\text { Vert.Grad. } \\
\text { (nT/ft) }\end{array}$} \\
\hline & & & & & & uncorr. & corr. & diff. & uncorr. & corr. & & \\
\hline 58 & 18 & 135556 & 1202 & 10 & 228.03 & 51459.2 & 51452.4 & 6.8 & 51456.4 & 51476.8 & -20.4 & -1.4 \\
\hline 58 & 18 & 135603 & 1204 & 10 & 233.03 & 51455.8 & 51449.0 & 6.8 & 51449.2 & 51469.6 & -20.4 & -3.3 \\
\hline 58 & 18 & 135610 & 1206 & 10 & 238.03 & 51454.0 & 51447.2 & 6.8 & 51452.4 & 51472.8 & $\mid-20.4$ & -0.8 \\
\hline 59 & 18 & 135815 & 1208 & 0 & 237.4 & 51451.8 & 51444.8 & 7.0 & 51332.6 & 51353.4 & -20.8 & -59.6 \\
\hline 59 & 18 & 135823 & 1210 & 0 & 232.4 & 51504.8 & 51497.7 & 7.1 & 51507.2 & 51528.1 & $\mid-20.9$ & 1.2 \\
\hline 59 & 18 & 135831 & 1212 & 0 & 227.4 & 51487.6 & 51480.5 & 7.1 & 51531.4 & 51552.3 & -20.9 & 21.9 \\
\hline 59 & 18 & 135839 & 1214 & 0 & 222.4 & 51467.8 & 51460.7 & 7.1 & 51478.6 & 51499.5 & $|-20.9|$ & 5.4 \\
\hline 59 & 18 & 135847 & 1216 & 0 & 217.4 & 51461.2 & 51454.1 & 7.1 & 51471.8 & 51492.7 & $|-20.9|$ & 5.3 \\
\hline 59 & 18 & 135854 & 1218 & 0 & 212.4 & 51457.4 & 51450.3 & 7.1 & 51464.2 & 51485.2 & -21.0 & 3.4 \\
\hline 59 & 18 & 135901 & 1220 & 0 & 207.4 & 51454.6 & 51447.5 & 7.1 & 51478.2 & 51499.2 & $|-21.0|$ & 11.8 \\
\hline 59 & 18 & 135908 & 1222 & 0 & 202.4 & 51453.4 & 51446.3 & 7.1 & 51473.8 & 51494.8 & $|-21.0|$ & 10.2 \\
\hline 59 & 18 & 135915 & 1224 & 0 & .197 .4 & 51448.0 & 51440.9 & 7.1 & 51455.0 & 51476.0 & $\mid-21.0$ & 3.5 \\
\hline 59 & 18 & 135922 & 1226 & 0 & 192.4 & 51447.2 & 51440.0 & 7.2 & 51457.8 & 51478.8 & -21.0 & 5.3 \\
\hline 59 & 18 & 135929 & 1228 & 0 & 187.4 & 51445.2 & 51438.0 & 7.2 & 51440.0 & 51461.1 & $|-21.1|$ & -2.6 \\
\hline 59 & 18 & 135935 & 1230 & 0 & 182.4 & 51440.0 & 51432.8 & 7.2 & 51390.0 & 11.1 & $|-21.1|$ & -25.0 \\
\hline 59 & 18 & 135943 & 1232 & 0 & 177.4 & 51702.4 & 51695.2 & 7.2 & .52193 .0 & 52214.1 & $\mid-21.1$ & 245.3 \\
\hline 59 & 18 & 135950 & 1234 & 0 & 172.4 & 51496.2 & 51489.0 & 7.2 & 51443.6 & 51464.7 & $|-21.1|$ & -26.3 \\
\hline 59 & 18 & 135957 & 1236 & 0 & 167.4 & 51491.8 & 51484.6 & 7.2 & 51526.2 & 51547.4 & $|-21.2|$ & 17.2 \\
\hline 59 & 18 & 140004 & 1238 & 0 & 162.4 & 51495.4 & 51488.2 & 7.2 & 51526.8 & 51548.0 & $\mid-21.2$ & 15.7 \\
\hline 5 & 18 & 140011 & 1240 & 0 & 157.4 & 51466.6 & 51459.4 & 7.2 & 51482.8 & 51504.0 & $|-21.2|$ & 8.1 \\
\hline
\end{tabular}


DAY 361 DIURNAL VARIATIONS

\begin{tabular}{|c|c|c|c|c|c|c|c|}
\hline \multicolumn{4}{|c|}{ TOP } & \multicolumn{4}{|c|}{ BOTTOM } \\
\hline Sta. & Time & Field & & Sta. & Time & Field & \\
\hline 1 & $13: 40: 30$ & 51495.6 & $13: 40: 46$ & 0 & 13:40:27 & 51495.7 & $13: 40: 43$ \\
\hline 3 & $13: 40: 38$ & 51495.5 & mean: 51 & 2 & 13:40:35 & 51495.7 & 5.9 \\
\hline 5 & $13: 40: 46$ & 51495.7 & sd: $c$ & 4 & $13: 40: 43$ & 51495.9 & sd \\
\hline 7 & $13: 40: 54$ & $51495: 8$ & & 6 & $13: 40: 51$ & 51496.0 & \\
\hline 9 & $13: 41: 02$ & 51495.8 & & 8 & $13: 40: 59$ & 51496.0 & \\
\hline 199 & $14: 07: 58$ & 51494.8 & $14: 08: 15$ & 198 & $-14: 07: 55$ & 51494.2 & 14:08:11 \\
\hline 201 & 14:08:06 & 51494.6 & mean: 51494.7. & 200 & 14:08:03 & 51494.2 & mean: $\mathbf{5 1 4 9 4 . 2}$ \\
\hline 203 & $14: 08: 15$ & 51494.5 & sd: 0.13 & 202 & $14: 08: 11$ & 51494.3 & sd: 0.04 \\
\hline 205 & $14: 08: 23$ & 51494.6 & & 204 & 14:08:20 & 51494.2 & \\
\hline 207 & $14: 08: 31$ & 51494.8 & & 206 & 14:08:28 & 51494.2 & \\
\hline 397 & $14: 34: 49$ & 51500.3 & 14:35:05 & 396 & $14: 34: 45$ & 51472.3 & 14:35:02 \\
\hline 399 & $14: 34: 57$ & 51500.4 & mean: 51500.2 & 398 & $14: 34: 54$ & 51499.1 & mean: 51 \\
\hline 401 & $14: 35: 05$ & 51500.2 & sd: 0.13 & 400 & $14: 35: 02$ & 51498.9 & sd: 11.92 \\
\hline 403 & $14: 35: 13$ & 51500.1 & & 402 & $14: 35: 10$ & 51498.9 & \\
\hline 405 & $14: 35: 21$ & 51500.1 & & 404 & $14: 35: 18$ & 51498.9 & \\
\hline 595 & $15: 05: 14$ & 51503.1 & $15: 05: 31$ & 594 & $15: 05: 11$ & 51499.8 & 15:05:27 \\
\hline 597 & $15: 05: 22$ & 51503.2 & mean: 51503.1. & 596 & $15: 05: 19$ & 51499.7 & mean: 51499.1 \\
\hline 599 & $15: 05: 31$ & 51503.1 & sd: 0.08 & 598 & $15: 05: 27$ & 51499.8 & sd: 0.15 \\
\hline 601 & $15: 05: 39$ & 51503.0 & & 600 & $15: 05: 35$ & 51499.9 & \\
\hline 603 & $15: 05: 47$ & 51503.2 & & 602 & $15: 05: 44$ & 51500.1 & \\
\hline 793 & $15: 29: 45$ & 51505.5 & $15: 30: 01$ & 792 & $15: 29: 42$ & 51500.5 & 15:29:58 \\
\hline 795 & $15: 29: 53$ & 51505.8 & mean: 515 & 794 & $15: 29: 50$ & 51500.4 & mean: 51500.4 \\
\hline 797 & $15: 30: 01$ & 51505.8 & sd: 0.19 & 796 & $15: 29: 58$ & 51500.5 & sd: 0.08 \\
\hline 799 & $15: 30: 09$ & 51505.5 & & 798 & $15: 30: 06$ & 51500.3 & \\
\hline 801 & $15: 30: 18$ & 51505.4 & & 800 & $15: 30: 14$ & 51500.4 & \\
\hline .991 & $15: 54: 37$ & 51486.5 & $15: 54: 54$ & 990 & $15: 54: 34$ & 51481.6 & $15: 54: 50$ \\
\hline 993 & $15: 54: 46$ & 51486.4 & mean: 51486.3 & 992 & $15: 54: 42$ & 51481.5 & mean: 51481.5 \\
\hline 995 & $15: 54: 54$ & 51486.4 & sd: 0.19 & 994 & $15: 54: 50$ & 51481.6 & sd: 0.08 \\
\hline 997 & $15: 55: 02$ & 51486.4 & & 996 & $15: 54: 59$ & 51481.5 & \\
\hline 999 & $15: 55: 10$ & 51486.0 & & 998 & $15: 55: 07$ & 51481.4 & \\
\hline
\end{tabular}




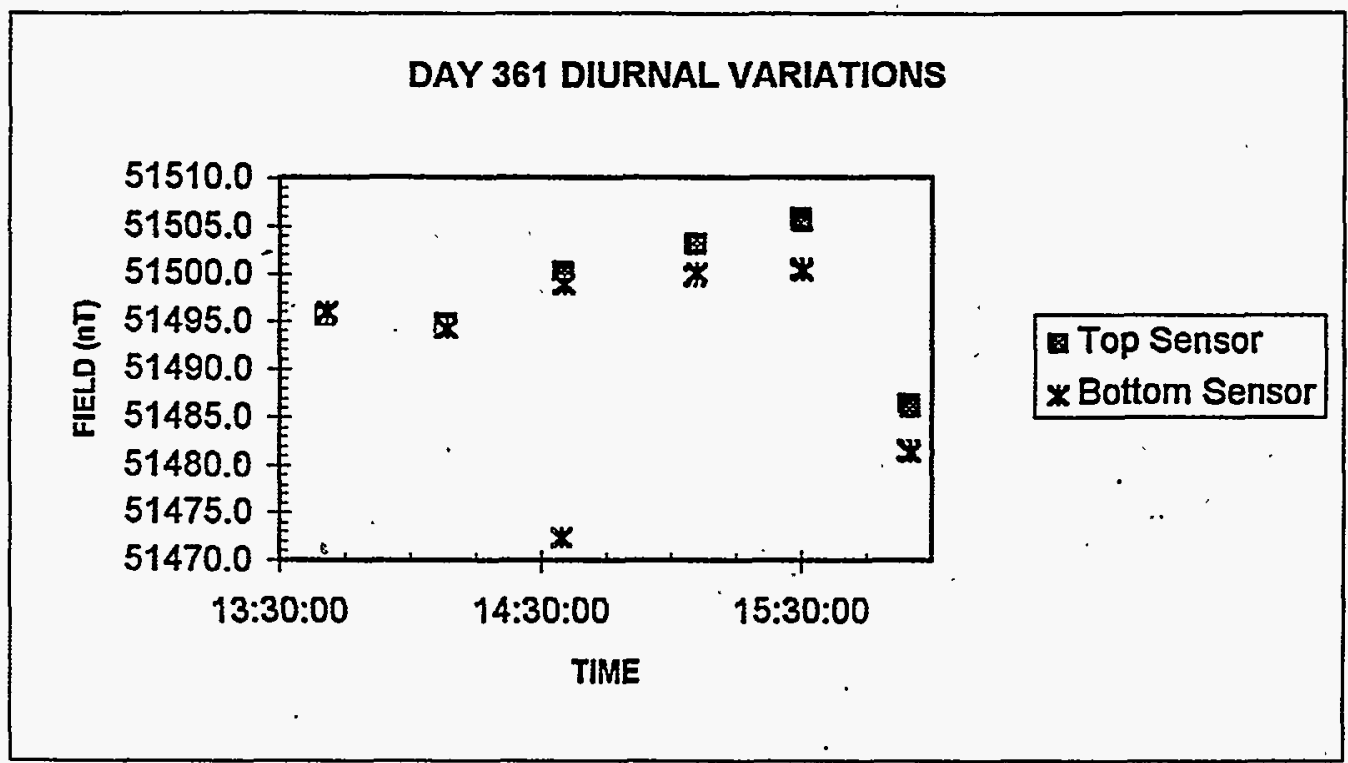

DAY 362 DIURNAL VARIATIONS

\begin{tabular}{|c|c|c|c|c|c|c|c|}
\hline \multicolumn{4}{|c|}{ TOP } & \multicolumn{4}{|c|}{ BOTTOM } \\
\hline Sta. & Time & Field (nT) & & Sta. & Time & Field (nT) & \\
\hline 1005 & $10: 53: 04$ & 51471.6 & $10: 53: 20$ & 1004 & $10: 53: 02$ & 51442.8 & $10: 53: 17$ \\
\hline 1007 & $10: 53: 12$ & 51472.0 & |mean: 51471.9 & 1006 & 10:53:10 & 51443.2 & mean: 51443.0 \\
\hline 1009 & $10: 53: 20$ & 51472.0 & sd: 0.18 & 1008 & $10: 53: 17$ & 51443.4 & sd: 0.26 \\
\hline 1011 & $10: 53: 26$ & 51472.0 & & 10.10 & 10:53:24 & 51442.8 & \\
\hline 1013 & $10: 53: 35$ & 51471.8 & & 1012 & $10: 53: 32$ & 51443.0 & \\
\hline 1203 & $11: 11: 40$ & 51465.6 & $11: 11: 53$ & 1202 & $11: 11: 37$ & 51436.6 & $11: 11: 51$ \\
\hline 1205 & $11: 11: 46$ & 51465.4 & mean: 51465.4 & 1204 & $11: 11: 44$ & 51436.2 & mean: 51436.1 \\
\hline 1207 & $11: 11: 53$ & 51465.2 & sd: 0.20 & 1206 & $11: 11: 51$ & 51436.0 & sd: 0.33 \\
\hline 1209 & $11: 12: 00$ & 51465.2 & & 1208 & 11:11:57 & 51435.8 & \\
\hline 1211 & $11: 12: 06$ & 51465.6 & & 1210 & $11: 12: 04$ & 51435.8 & \\
\hline$\overline{1401}$ & $11: 35: 09$ & 51463.4 & $11: 35: 24$ & 1400 & $11: 35: 07$ & 51432.2 & 11:35:21 \\
\hline 1403 & $11: 35: 16$ & 51463.0 & mean: 51463.2 & 1402 & $11: 35: 14$ & 51432.2 & mean: 51432.4 \\
\hline 1405 & $11: 35: 24$ & 51463.0 & sd: 0.30 & 1404 & $11: 35: 22$ & 51432.6 & sd: 0.22 \\
\hline 1407 & $11: 35: 31$ & 51463.6 & & 1406 & $11: 35: 28$ & 51432.6 & \\
\hline 1409 & $11: 35: 37$ & 51463.6 & & 1408 & $11: 35: 35$ & 51432.6 & \\
\hline 1599 & $11: 55: 49$ & 51455.0 & $11: 56: 03$ & 1598 & $11: 55: 47$ & 51432.0 & $11: 56: 00$ \\
\hline 1601 & $11: 55: 56$ & 51455.4 & mean: 51455.8 & 1600 & $11: 55: 54$ & 51432.0 & mean: 51432.8 \\
\hline 1603 & $11: 56: 03$ & 51456.2 & sd: 0.61 & 1602 & $11: 56: 00$ & 51433.6 & sd: 0.75 \\
\hline 1605 & $11: 56: 09$ & 51456.4 & & 1604 & $11: 56: 07$ & 51433.4 & \\
\hline 1607 & $11: 56: 16$ & 51456.2 & & 1606 & $11: 56: 14$ & 51432.8 & \\
\hline 1609 & $13: 17: 38$ & 51474.4 & $13: 17: 48$ & 1608 & $13: 17: 36$ & 51445.4 & $13: 17: 47$ \\
\hline 1611 & $13: 17: 43$ & 5.1474 .6 & mean: 51474.5 & 1610 & $13: 17: 40$ & 51445.2 & mean: 51445.2 \\
\hline 1613 & $13: 17: 49$ & 51474.0 & sd: 0.36 & 1612 & $13: 17: 47$ & 51445.4 & sd: 0.26 \\
\hline 1615 & $13: 17: 56$ & 51475.0 & & 1614 & $13: 17: 53$ & 51444.8 & \\
\hline 1617 & 13:18:02 & 51474.4 & & 1616 & $13: 18: 00$ & 51445.0 & \\
\hline 1807 & $13: 35: 49$ & 51474.6 & $13: 36: 02$ & 1806 & $13: 35: 47$ & 51447.6 & $13: 36: 00$ \\
\hline 1809 & $13: 35: 56$ & 51475.2 & mean: 51475.4 & 1808 & 13:35:53 & 51447.2 & mean: 51447.4 \\
\hline 1811 & 13:36:02 & 51475.8 & sd: 0.54 & 1810 & 13:36:00 & 51447.4 & sd: 0.14 \\
\hline
\end{tabular}




\begin{tabular}{|c|c|c|c|c|c|c|c|}
\hline $\begin{array}{l}1813 \\
1815\end{array}$ & $\begin{array}{l}13: 36: 09 \\
13: 36: 16\end{array}$ & $\begin{array}{l}51475.8 \\
51475.8\end{array}$ & & $\begin{array}{l}1812 \\
1814\end{array}$ & $\begin{array}{l}13: 36: 07 \\
13: 36: 13\end{array}$ & $\begin{array}{l}51447.4 \\
51447.4\end{array}$ & \\
\hline 2005 & $14: 00: 22$ & 51482.0 & $14: 00: 37$ & 2004 & $14: 00: 19$ & 51448.4 & $14: 00: 35$ \\
\hline 2007 & $14: 00: 26$ & 51482.2 & mean: 51482.6 & 2006 & 14:00:24 & 51448.2 & mean: 51448.2 \\
\hline 2009 & $14: 00: 33$ & 51482.4 & sd: 0.53 & 2008 & $14: 00: 31$ & 51448.6 & sd: 0.34 \\
\hline 2011 & $14: 00: 39$ & 51483.0 & & 2010 & $14: 00: 37$ & 51448.2 & \\
\hline 2013 & $14: 00: 47$ & 51483.4 & & 2012 & $14: 00: 45$ & 51448.4 & \\
\hline 2015 & $14: 00: 54$ & 51482.4 & & 2014 & $14: 00: 51$ & 51447.6 & \\
\hline 2205 & $14: 22: 42$ & 51486.2 & $14: 22: 55$ & 2204 & $14: 22: 39$ & 51452.8 & $14: 22: 53$ \\
\hline 2207 & $14: 22: 48$ & 51486.8 & mean: $\mathbf{5 1 4 8 6 . 3}$ & 2206 & 14:22:46 & 51452.0 & mean: 51452.1 \\
\hline 2209 & $14: 22: 55$ & 51486.8 & sd: 0.46 & 2208 & $14: 22: 53$ & 51452.2 & sd: 0.44 \\
\hline 2211 & $14: 23: 02$ & 51486.0 & & 2210 & $14: 23: 00$ & 51452.0 & \\
\hline 2213 & $14: 23: 09$ & 51485.8 & & 2212 & 14:23:06 & 51451.6 & \\
\hline 2403 & $14: 47: 42$ & 51487.6 & $14: 47: 55$ & 2402 & $14: 47: 40$ & 51454.8 & $14: 47: 53$ \\
\hline 2405 & $14: 47: 49$ & 51487.6 & mean: 51487.7 & 2404 & $14: 47: 47$ & 51454.6 & mean: 51454.6 \\
\hline 2407 & $14: 47: 55$ & 51487.8 & sd: 0.11 & 2406 & $14: 47: 53$ & 51454.8 & sd: 0.17 \\
\hline 2409 & $14: 48: 02$ & 51487.6 & & 2408 & $14: 48: 00$ & 51454.4 & \\
\hline 2411 & $14: 48: 09$ & 51487.8 & & 2410 & 14:48:08 & 51454.6 & \\
\hline 2601 & $15: 09: 08$ & 51496.0 & 15:09:22 & 2600 & 15:09:06 & 51456.8 & 15:09:19 \\
\hline 2603 & 15:09:15 & 51496.0 & mean: 51496.0 & 2602 & $15: 09: 13$ & 51456.8 & mean: 51456.8 \\
\hline 2605 & 15:09:22 & 51496.0 & sd: 0.09 & 2604 & 15:09:19 & 51456.8 & sd: 0.09 \\
\hline 2607 & $15: 09: 28$ & 51496.0 & & 2606 & $15: 09: 26$ & 51457.0 & \\
\hline 2609 & $15: 09: 35$ & 51496.2 & & 2608 & 15:09:33 & 51456.8 & \\
\hline
\end{tabular}

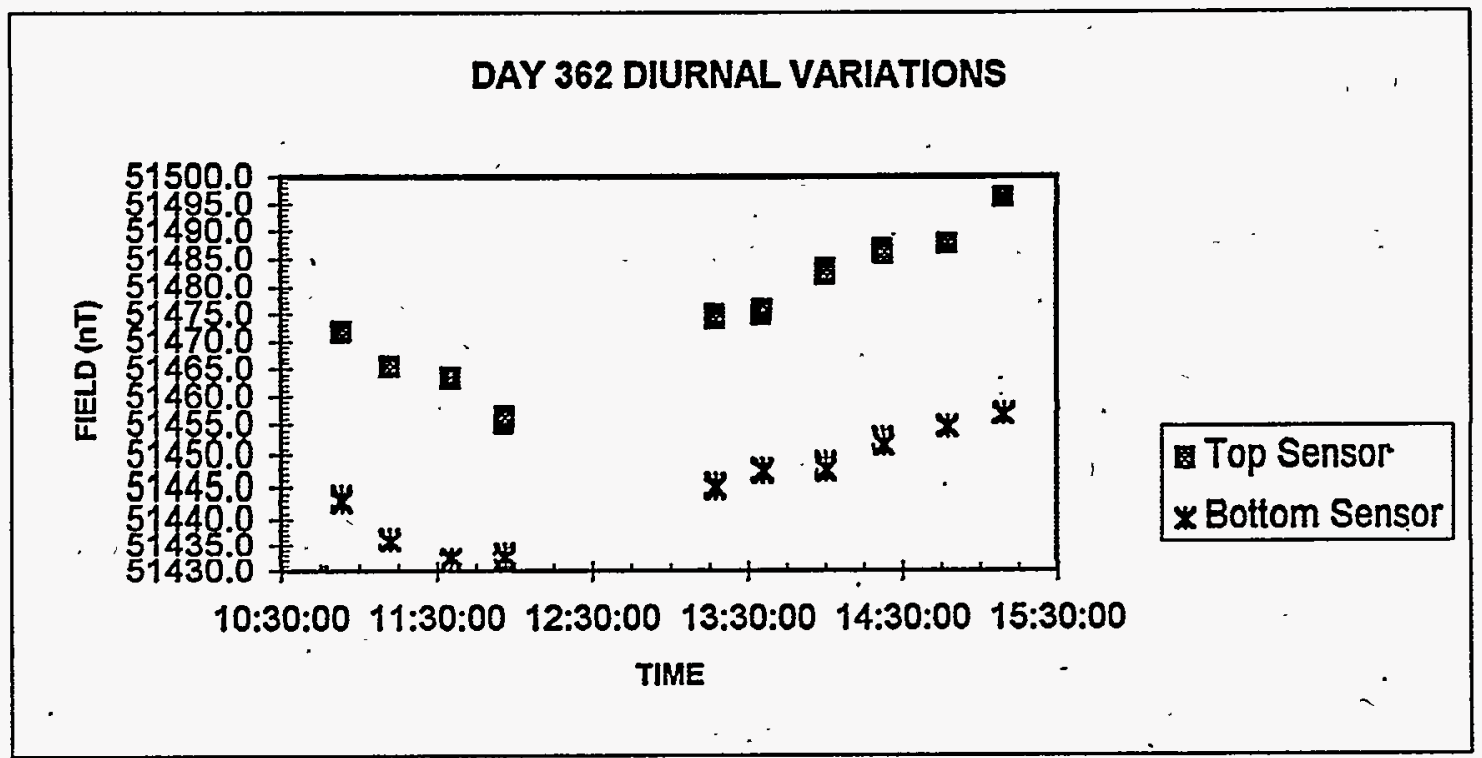

DAY 17 DIURNAL VARIATIONS

\begin{tabular}{|r|l|l|l|l|l|l|l|}
\hline \multicolumn{3}{|c|}{ TOP } & \multicolumn{3}{c|}{ BOTTOM } \\
\hline Sta. & Time & Field (nT) & Sta. & \multicolumn{1}{|c|}{ Time } & Field (nT) & \\
\hline 1 & $9: 53: 16$ & 51307.8 & $9: 53: 29$ & 0 & $9: 53: 14$ & 51240.0 & $9: 53: 26$ \\
3 & $9: 53: 22$ & 51307.2 & mean: 51307.0 & 2 & $9: 53: 20$ & 51239.8 & mean: $\mathbf{5 1 2 3 9 . 6}$ \\
5 & $9: 53: 29$ & 51306.4 & sd: 0.55 & 4 & $9: 53: 26$ & 51239.4 & sd: 0.26 \\
7 & $9: 53: 35$ & 51307.0 & & 6 & $9: 53: 33$ & 51239.4 & \\
9 & $9: 53: 41$ & 51306.6 & & 8 & $9: 53: 39$ & 51239.6 & \\
\hline
\end{tabular}




\begin{tabular}{|c|c|c|c|c|c|c|c|}
\hline $\begin{array}{l}247 \\
249 \\
251 \\
253 \\
255\end{array}$ & $\begin{array}{l}10: 15: 57 \\
10: 16: 03 \\
10: 16: 09 \\
10: 16: 15 \\
10: 16: 22\end{array}$ & $\begin{array}{l}51302.6 \\
51302.6 \\
51303.0 \\
51302.8 \\
51303.4\end{array}$ & $\begin{array}{l}\text { 10:16:09 } \\
\text { mean: } 51302.9 \\
\text { sd: } 0.33\end{array}$ & $\begin{array}{l}246 \\
248 \\
250 \\
252 \\
254\end{array}$ & $\begin{array}{c}10: 15: 55 \\
10: 16: 01 \\
10: 16: 07 \\
10: 16: 13 \\
10: 16: 19\end{array}$ & $\begin{array}{l}51231.4 \\
51231.6 \\
51232.2 \\
51232.0 \\
51232.4\end{array}$ & $\begin{array}{l}\text { 10:16:07 } \\
\text { mean: } 51231.9 \\
\text { sd: } 0.41\end{array}$ \\
\hline 501 & $10: 44: 31$ & 51301.6 & $10: 44: 43$ & 500 & $10: 44: 28$ & 51231.0 & $10: 44: 41$ \\
\hline 503 & $10: 44: 37$ & 51301.4 & mean: 513 & 502 & $10: 44: 35$ & 51230.8 & mean: 51 \\
\hline 505 & $10: 44: 43$ & 51301.2 & sd: 0.55 & 504 & $10: 44: 41$ & 51231.4 & sd: 0.41 \\
\hline 507 & $10: 44: 49$ & 51300.8 & & 506 & $10: 44: 47$ & 51231.6 & \\
\hline 509 & $10: 44: 55$ & 51300.2 & & 508 & $10: 44: 53$ & .51230 .6 & \\
\hline 511 & $11: 52: 59$ & 51271.8 & 11:56:05 & 510 & 11:52:57 & 51202.4 & 11:56:03 \\
\hline 513 & $11: 56: 42$ & 51270.6 & mean: 51271.4 & 512 & $11: 56: 40$ & 51201.6 & mea \\
\hline 515 & $11: 56: 48$ & 51270.6 & sd: 0.78 & 514 & $11: 56: 46$ & 51202.0 & sd: 0.4 \\
\hline 517 & $11: 56: 55$ & 51271.4 & & 516 & $11: 56: 53$ & 51201.6 & \\
\hline 519 & $11: 57: 02$ & 51272.4 & & 518 & $11: 56: 59$ & 51201.2 & \\
\hline 771 & $12: 26: 41$ & 51264.2 & 12:26:53 & 770 & $12: 26: 38$ & 51195.4 & $12: 26: 50$ \\
\hline 773 & $12: 26: 47$ & 51264.6 & mean: 51264.7 & 772 & $12: 26: 44$ & 51195.0 & Imean: 51 \\
\hline 775 & $12: 26: 53$ & 51265.4 & sd: 0.48 & 774 & $12: 26: 50$ & 51195.0 & sd: 0.27 \\
\hline 777 & $12: 26: 59$ & 51265.0 & & 776 & $12: 26: 56$ & 51194.8 & \\
\hline 779 & 12:27:05 & 51264.4 & & 778 & 12:27:02 & 51195.4 & \\
\hline 1037 & $13: 14: 10$ & 51279.0 & $13: 14: 23$ & 1036 & $13: 14: 08$ & 51205.6 & $13: 14: 21$ \\
\hline 1039 & $13: 14: 17$ & 51278.6 & mean: 51278.3 & 1038 & $13: 14: 14$ & 51204.6 & mean: 51 \\
\hline 1041 & 13:14:23 & 51278.6 & sd: 0.69 & 1040 & $13: 14: 21$ & 51204.4 & \\
\hline 1043 & $13: 14: 30$ & 51278.2 & & 1042 & $13: 14: 28$ & 51204.8 & \\
\hline 1045 & $13: 14: 37$ & 51277.2 & & 1044 & $13: 14: 34$ & 51204.4 & \\
\hline 1307 & 13:52:23 & 51271.4 & $13: 52: 37$ & 1306 & $13: 52: 20$ & 51155.2 & $13: 52: 34$ \\
\hline 1309 & $13: 52: 30$ & 51270.8 & mean: $\$ 1271.4$ & 1308 & $13: 52: 28$ & 51210.0 & mean: $\mathbf{5 1 1 9 8 . 9}$ \\
\hline 1311 & $13: 52: 37$ & 51270.8 & sd: 0.73 & 1310 & $13: 52: 34$ & $\cdot 51210.0$ & sd: .24 .42 \\
\hline 1313 & $13: 52: 43$ & 51271.4 & & 1312 & $13: 52: 41$ & 51209.6 & \\
\hline 1315 & $13: 52: 50$ & 51272.6 & & 1314 & $13: 52: 48$ & .51209 .6 & \\
\hline 1529 & $14: 26: 21$ & 51279.6 & $14: 26: 34$ & 1528 & $14: 26: 18$ & 51219.6 & $14: 26: 32$ \\
\hline 1531 & $14: 26: 27$ & 51279.4 & mean: 51279.6 & 1530 & $14: 26: 25$ & 51219.8 & mean: 51220.1 \\
\hline 1533 & $14: 26: 34$ & 51279.6 & sd: 0.17 & 1532 & $14: 26: 32$ & 51219.6 & sd: 1.05 \\
\hline 1535 & $14: 26: 41$ & 51279.8 & & .1534 & $14: 26: 39$ & 51219.6 & \\
\hline 1537 & $14: 26: 48$ & 51279.8 & & 1536 & $14: 26: 46$ & 51222.0 & \\
\hline 1695 & $14: 50: 05$ & 51288.0 & $14: 50: 13$ & 1694 & $14: 50: 03$ & 51222.0 & 14:50:16 \\
\hline 1697 & $14: 50: 12$ & $51288: 2$ & mean: 51288.1 & 1696 & $14: 50: 10$ & 51222.6 & mean: 51223.3 \\
\hline 1699 & $14: 50: 19$ & 51287.6 & sd: 0.91 & 1698 & $14: 50: 16$ & 51222.2 & sd: 2.20 \\
\hline 1701 & $14: 50: 26$ & 51287.2 & & 1700 & $14: 50: 23$ & 51222.4 & \\
\hline 1703 & $14: 50: 32$ & 51289.6 & & 1702 & $14: 50: 30$ & 51227.2 & \\
\hline
\end{tabular}




\section{DAY 17 DIURNAL VARIATIONS}

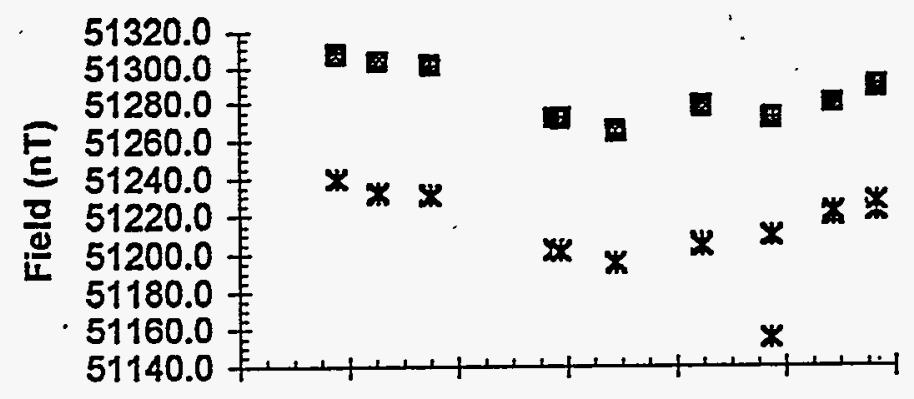

9:00:0 10:00: 11:00: 12:00: 13:00: 14:00: 15:00:

$\begin{array}{lllllll}0 & 00 & 00 & 00 & 00 & 00 & 00\end{array}$

Time

DAY 18 DIURNAL VARIATIONS

\begin{tabular}{|c|c|c|c|c|c|c|c|}
\hline \multicolumn{4}{|c|}{ TOP } & \multicolumn{4}{|c|}{ BOTTOM } \\
\hline Sta. & Time & Field (nT) & & Sta. & Time & Field (nT) & \\
\hline 1 & $11: 12: 53$ & 51643.8 & $11: 13: 06$ & 0 & $11: 12: 50$ & 51533.6 & $11: 13: 04$ \\
\hline 3 & $11: 13: 00$ & 51641.0 & mean: 51640.8 & 2 & 11:12:58 & 51533.4 & mean: 51522.2 \\
\hline 5 & $11: 13: 06$ & 51639.2 & sd: 1.77 & 4 & $11: 13: 04$ & 51533.0 & sd: 0.26 \\
\hline 7 & $11: 13: 13$ & 51640.0 & & 6 & $11: 13: 10$ & 51533.0 & \\
\hline 9 & $11: 13: 19$ & 51640.2 & & 8 & $11: 13: 17$ & 51533.2 & \\
\hline 159 & $11: 27: 23$ & 51641.6 & $11: 27: 35$ & 158 & $11: 27: 20$ & 51534.4 & $11: 27: 33$ \\
\hline 161 & $11: 27: 29$ & 51640.6 & mean: 51646.2 & 160 & $11: 27: 27$ & 51534.6 & mean: 51535.2 \\
\hline 163 & $11: 27: 35$ & 51641.8 & sd: 9.87 & 162 & $11: 27: 33$ & 51535.0 & sd: 0.82 \\
\hline$\uparrow 65$ & $11: 27: 41$ & 51643.4 & & 164 & $11: 27: 39$ & 51536.2 & \\
\hline 167 & $11: 27: 47$ & 51663.8 & & 166 & $11: 27: 45$ & 51536.0 & \\
\hline 325 & $11: 47: 23$ & 51634.6 & $11: 47: 37$ & 324| & $11: 47: 21$ & 51535.0 & $11: 47: 35$ \\
\hline 327 & $11: 47: 29$ & 51638.6 & mean: $\mathbf{5 1 6 3 4 . 3}$ & 326 & $11: 47: 27$ & 51536.2 & mean: 51537.1 \\
\hline 329 & $11: 47: 35$ & 51623.4 & sd: 7.54 & 328 & $11: 47: 33$ & 51537.0 & sd: 1.65 \\
\hline 331 & $11: 47: 46$ & 51643.4 & & 330 & $11: 47: 44$ & 51539.2 & \\
\hline 333 & $11: 47: 52$ & 51631.6 & & 332 & $11: 47: 49$ & 51538.2 & \\
\hline 491 & $12: 07: 13$ & 51646.4 & $12: 07: 26$ & 490 & $12: 07: 11$ & 51539.2 & 12:07:23 \\
\hline 493 & $12: 07: 19$ & 51650.6 & mean: 51649.0 & 492 & 12:07:17 & 51540.4 & mean: 51540.0 \\
\hline 495 & $12: 07: 26$ & 51648.4 & sd: 1.66 & 494 & $12: 07: 23$ & 51539.8 & sd: 0.55 \\
\hline 497 & $12: 07: 32$ & 51649.8 & & 496 & $12: 07: 29$ & 51539.8 & \\
\hline 499 & $12: 07: 38$ & 51649.8 & & 498 & $12: 07: 35$ & 51540.6 & \\
\hline 657 & $12: 29: 20$ & 51646.0 & $12: 29: 33$ & 656 & $12: 29: 18$ & 51541.0 & $12: 29: 30$ \\
\hline 659 & $12: 29: 26$ & 51645.4 & mean: 51646.8 & 658 & 12:29:24 & 51541.8 & mean: 51541.6 \\
\hline 661 & $12: 29: 33$ & 51646.2 & sd: 1.45 & 660 & 12:29:30 & 51541.0 & sd: 0.57 \\
\hline 663 & $12: 29: 39$ & 51647.6 & & 662 & 12:29:36 & 51542.2 & \\
\hline 665 & $12: 29: 45$ & 51649.0 & & 664 & $12: 29: 42$ & 51542.0 & \\
\hline 809 & $12: 58: 48$ & 51651.4 & 12:59:00 & 808 & $12: 58: 46$ & 51536.0 & $12: 58: 58$ \\
\hline 811 & $12: 58: 54$ & 51649.2 & mean: 51647.5 & 810 & $12: 58: 52$ & 51535.8 & |mean: 51536.1 \\
\hline
\end{tabular}




\begin{tabular}{r|l|l|l|r|r|l|l|}
813 & $12: 59: 00$ & 51648.2 & sd: 3.09 & 812 & $12: 58: 58$ & 51537.0 & sd: $\mathbf{0 . 5 4}$ \\
815 & $12: 59: 06$ & 51644.8 & & 814 & $12: 59: 04$ & 51535.6 & \\
817 & $12: 59: 12$ & 51644.0 & & 816 & $12: 59: 10$ & 51536.0 & \\
\hline 1039 & $13: 28: 30$ & 51652.4 & $13: 28: 44$ & 1038 & $13: 28: 28$ & 51544.4 & $\mathbf{1 3 : 2 8 : 4 1}$ \\
1041 & $13: 28: 37$ & 51652.4 & mean: 51652.2 & 1040 & $13: 28: 35$ & 51545.0 & mean: $\mathbf{5 1 5 4 4 . 5}$ \\
1043 & $13: 28: 44$ & 51652.2 & sd: 0.15 & 1042 & $13: 28: 41$ & 51545.8 & sd: 0.92 \\
1045 & $13: 28: 50$ & 51651.6 & & 1044 & $13: 28: 48$ & 51544.0 & \\
1047 & $13: 28: 57$ & 51652.4 & & 1046 & $13: 28: 55$ & 51543.4 & \\
\hline 1243 & $14: 03: 33$ & 51654.6 & $14: 03: 47$ & 1242 & $14: 03: 31$ & 51536.6 & $14: 03: 44$ \\
1245 & $14: 03: 40$ & 51653.8 & mean: 51655.7 & 1244 & $14: 03: 37$ & 51536.8 & mean: $\mathbf{5 1 5 3 7 . 7}$ \\
1247 & $14: 03: 47$ & 51655.6 & sd: 1.76 & 1246 & $14: 03: 44$ & 51536.8 & sd: 1.45. \\
1249 & $14: 03: 54$ & 51656.2 & & 1248 & $14: 03: 51$ & 51538.2 & \\
1251 & $14: 04: 00$ & 51658.4 & & 1250 & $14: 03: 58$ & $\cdot 51540.0$ & \\
\hline
\end{tabular}

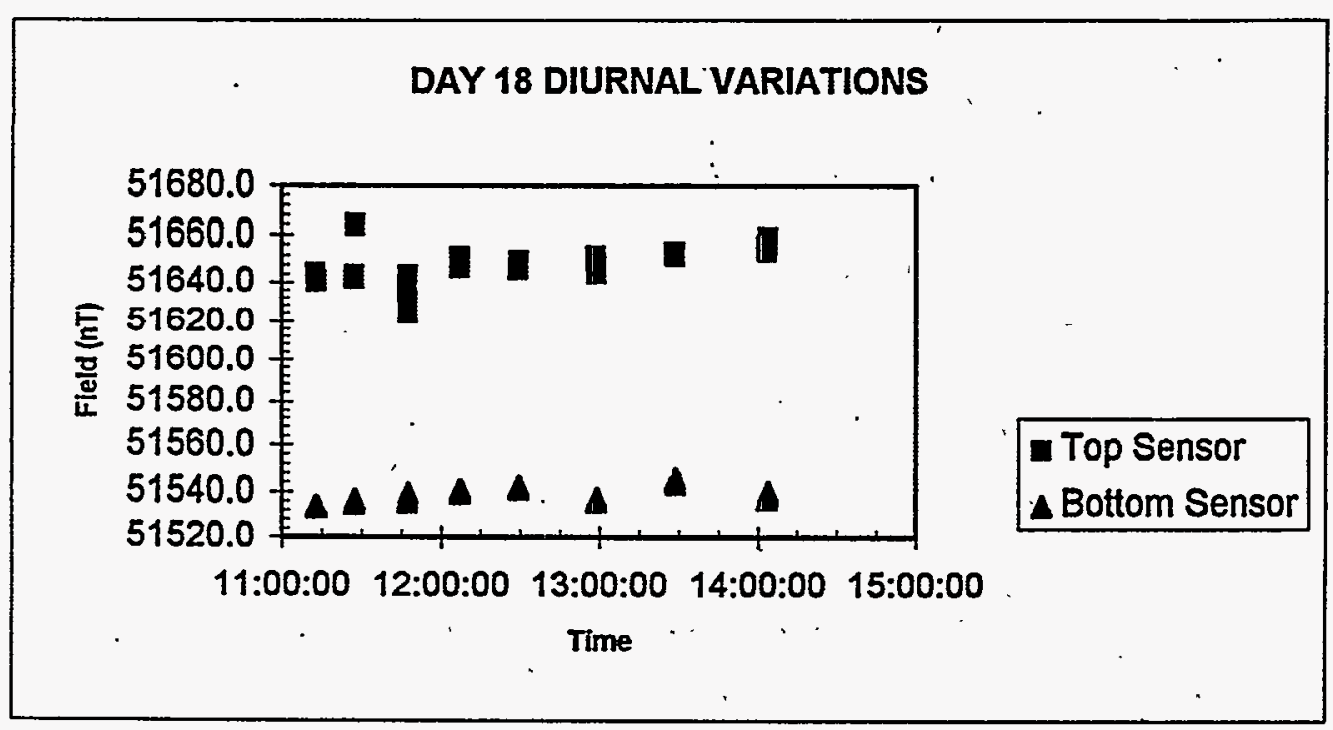

